\title{
IMPLEMENTATION AND ASSESSMENT OF HYPERGLYCEMIC CONDITIONS FOR THE CREATION OF A DIABETIC BLOOD VESSEL MIMIC
}

\author{
A Thesis \\ Presented to \\ the Faculty of California Polytechnic State University, \\ San Luis Obispo
}

\author{
In Partial Fulfillment \\ of the Requirements for the Degree \\ Master of Science in Biomedical Engineering
}

By

Vikramaditya Mediratta

June 2011 
(C) 2011

Vikramaditya Mediratta

All Rights Reserved 


\section{COMMITTEE MEMBERSHIP}

TITLE:

IMPLEMENTATION AND ASSESSMENT OF HYPERGLYCEMIC CONDITIONS FOR THE CREATION OF A DIABETIC BLOOD

VESSEL MIMIC

AUTHOR: $\quad$ Vikramaditya Mediratta

DATE SUBMITTED: June 2011

Dr. Kristen O'Halloran Cardinal

Committee Chair

Dr. Trevor R. Cardinal

Committee Member

Dr. David S. Clague

Committee Member 


\begin{abstract}
IMPLEMENTATION AND ASSESSMENT OF HYPERGLYCEMIC CONDITIONS FOR THE CREATION OF A DIABETIC BLOOD VESSEL MIMIC
\end{abstract}

By Vikramaditya Mediratta

Introduction: Diabetes Mellitus is a metabolic disorder that affects a person's ability to either produce insulin (Type I diabetes mellitus) or properly use insulin (Type II diabetes mellitus) in order to maintain adequate blood glucose levels. The most severe diabetic complications arise due to hyperglycemia - a state of extremely high blood glucose levels - such as, coronary artery disease (CAD), in which coronary stent therapy is a popular method of treatment. However, research has shown a high rate of in-stent restenosis in diabetic patients with CAD, most likely due to activation of cellular adhesion molecules on endothelial cells exposed to the hyperglycemic environment. Blood vessel mimics (BVMs) have been researched as viable options for in vitro studies on vascular stents; thus, it would be beneficial to create an in vitro diabetic BVM for stent manufactures to evaluate and determine the root cause of the high failure rate of stents in the diabetic population. In addition, a diabetic BVM would help manufactures optimize coatings or stent configurations for diabetic patients. Methods: The purpose of this thesis was to take the initial steps towards the goal of a diabetic BVM. The first aim was to establish a procedure of developing glycemic cell media solutions of various glucose concentrations, and to establish a feasible method of monitoring the glucose concentration of the solutions. Glycemic cell media solutions were developed and their glucose concentrations were evaluated with a blood glucose meter (specifically, the Aviva Accu-Chek blood glucose meter) 
or visual blood glucose test strips (Glucoflex R visual blood glucose test strips). The second aim was to ensure that the developed glycemic cell media solutions could be monitored in a cell culture environment over time, and to determine if the hyperglycemic conditions induced any change to endothelial cells. Bovine aortic endothelial cells (BAECs) and human umbilical vein endothelial cells (HUVECs) were used to evaluate glucose consumption and cell morphology. Glucose concentration of the cell media was recorded to evaluate glucose consumption, and the cells were evaluated under a microscope in order to determine cell morphology and an increase in cell death. Results \& Conclusions: Data accumulated from the first set of experiments confirmed that glycemic cell media solutions can be developed by adding Sigma G6512 D-(+)glucose to base cell media. Additionally, the Aviva Accu-Chek blood glucose meter recorded the most accurate and precise glucose concentrations of the various glycemic cell media solutions compared to the Glucoflex-R blood glucose visual test strips. Lastly, the series of experiments with BAECs and HUVECs confirmed that the glycemic cell media solutions could be effectively monitored over time, and that these conditions evoked higher glucose consumption by the endothelial cells compared to the normal glycemic cell media solutions. Additionally, neither glycemic environment evoked significant cell death. These results met the aims of this thesis, and therefore provide the foundation for further development of a diabetic BVM. 


\section{Acknowledgements}

There are many people that I would like to thank. First, I must thank Dr. Kristen O'Halloran Cardinal for her continuous dedication and leadership to help me strive for my goals and achieve my dream of earning a Master of Science. Without her expertise and devotion to her research, this thesis would not be possible. It has been an absolute privilege working and learning from you.

I would also like to thank the faculty members of my thesis committee - Dr. Trevor R. Cardinal and Dr. David S. Clague. Without their generosity and interest, the completion of this thesis would not be possible. Thank you for having such interest in the thesis I put so much work into.

Additionally, I would like to thank my fellow lab members of the Tissue Engineering lab. It was a privilege and joy working with everyone and being a part of such a dedicated and collaborative group of individuals. I would especially like to extend my gratitude to Brian Wong and Marcus Foley. Their willingness to help at all necessary times was a significant factor in my completion of this thesis.

Lastly, but most importantly, I want to thank my parents, Subash \& Rashmi Mediratta. Their love, support and encouragement helped me pursue a higher education and make the most of the opportunities presented to me. Thank you for pushing me to do my best whenever my best was needed. My achievements are and will always be a reflection of your love and support. 


\section{Table of Contents}

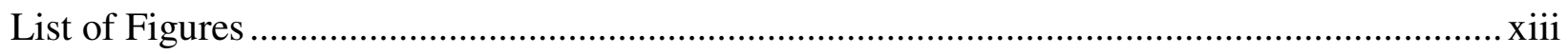

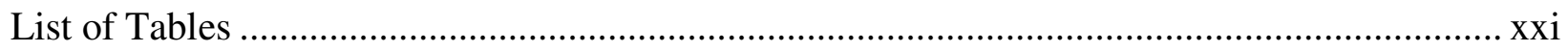

Chapter 1: An Introduction to Diabetes Mellitus .................................................................. 1

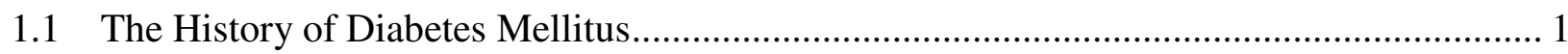

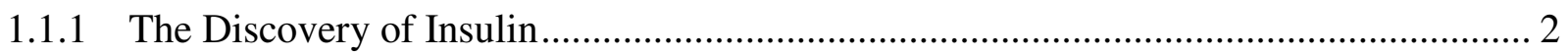

1.1.2 Impact of the Discovery of Insulin.......................................................................... 5

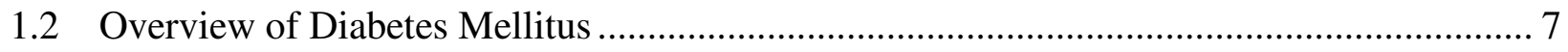

1.2.1 Type I (Insulin Deficient) Diabetes Mellitus ............................................................... 8

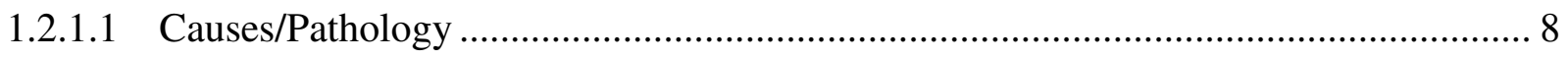

1.2.2 Type 2 (Insulin Resistant) Diabetes Mellitus ............................................................. 10

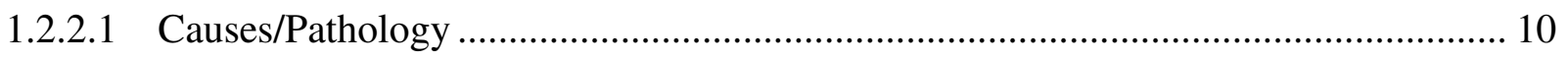

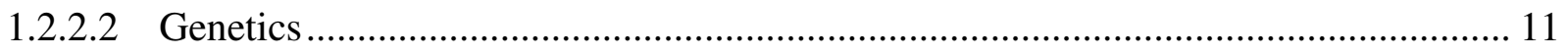

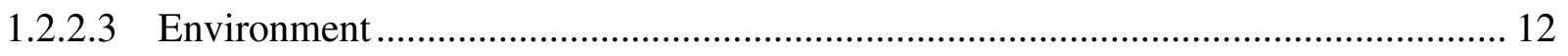

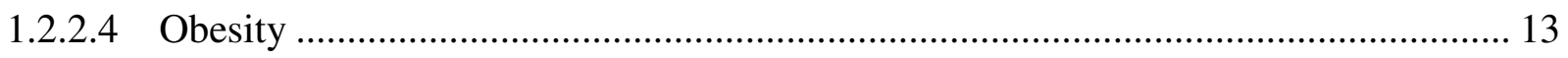

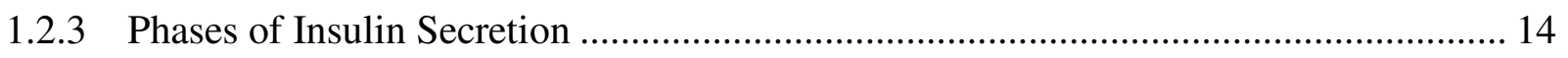

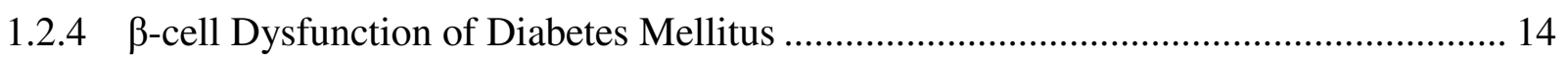




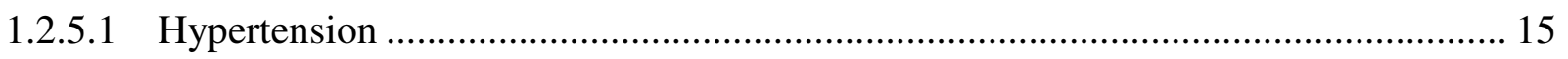

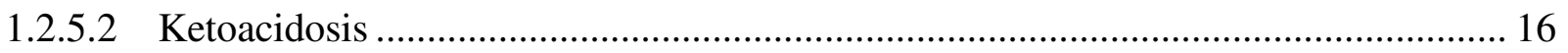

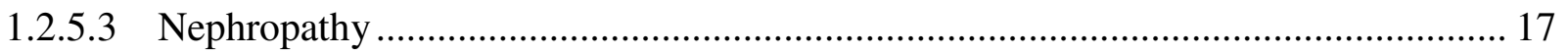

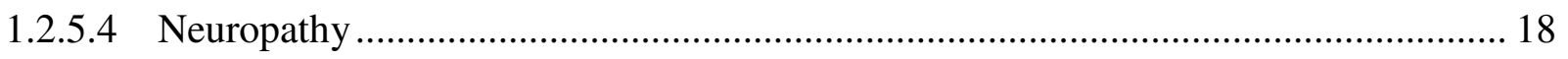

1.2.5.5 Cardiovascular Disease ........................................................................... 19

1.2.5.6 Coronary Artery Disease ............................................................................. 19

1.3 Treatments for Diabetics with Coronary Artery Disease ........................................... 21

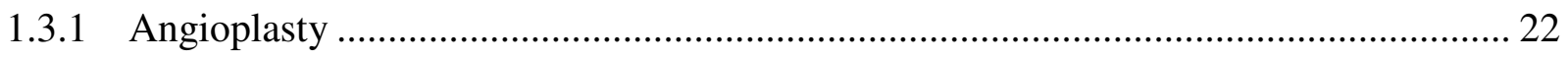

1.3.2 Coronary Artery Bypass Surgery ...................................................................... 25

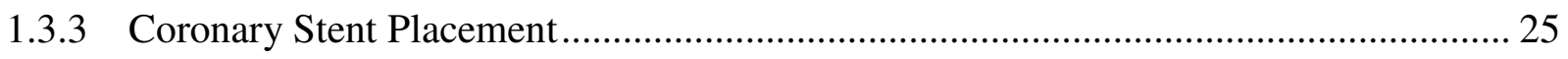

1.4 Coronary Stent Treatment in the Diabetic Population ............................................. 28

1.4.1 Restenosis in In Vivo Diabetic Studies............................................................. 28

1.4.2 Clinical Results of Diabetic Coronary Stent Implantation ..................................... 30

1.4.2.1 Type I Diabetics vs. Type 2 Diabetics ......................................................... 30

1.4.2.2 Bare Metal Stents vs. Drug-Eluting Stents in the Diabetic Population..................... 31

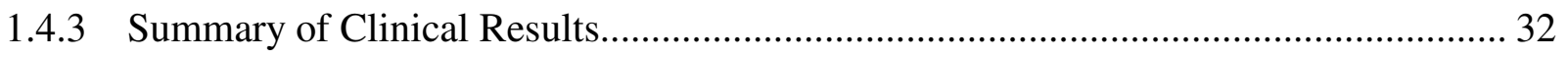




\section{Chapter 2: Establishment \& Evaluation of High Glucose Solutions in Order to Create

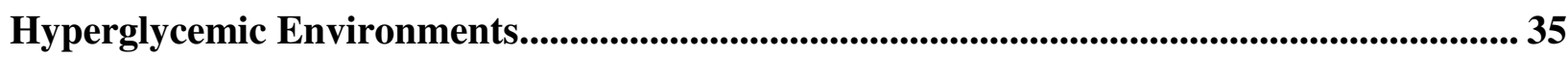

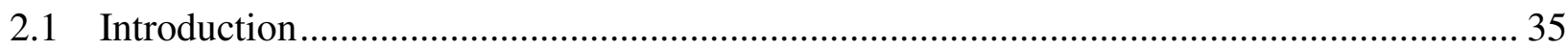

2.1.1 Significance of Hyperglycemia \& Common Complications in Diabetic Patients ........ 36

2.1.2 Vascular Damage Induced by Hyperglycemia................................................... 38

2.1.2.1 Molecular Mechanisms of Glucose-Mediated Vascular Damage ........................... 40

2.1.3 Blood Glucose ............................................................................................... 44

2.1.3.1 Properties of Glucose ................................................................................... 44

2.1.3.2 Normal Blood Glucose Levels .................................................................. 47

2.1.3.3 Metabolism \& Regulation of Glucose.......................................................... 49

2.1.3.4 Dysfunction of Glucose Metabolism \& Insulin Signaling .................................... 53

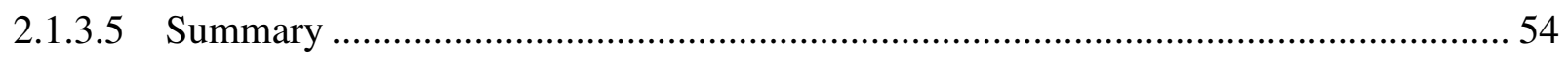

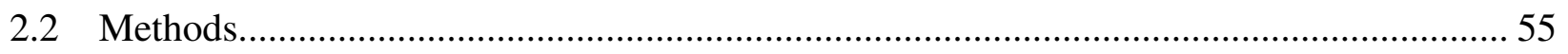

2.2.1 Methods of Measuring and Monitoring Blood Glucose.......................................... 56

2.2.1.1 Blood Glucose Meter ....................................................................................... 56

2.2.1.2 Continuous Glucose Monitoring ................................................................... 59

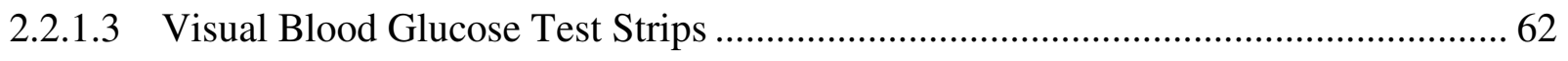
viii 
2.2.1.4 Glucose sensing bio-implants.

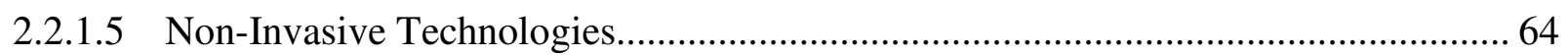

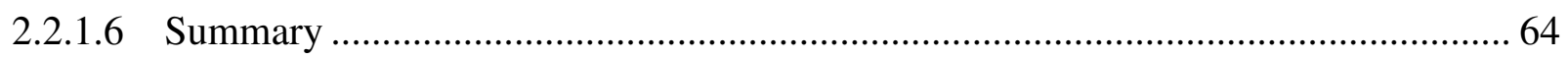

2.2.2 Methods of Producing and Monitoring Different Glycemic Solutions....................... 65

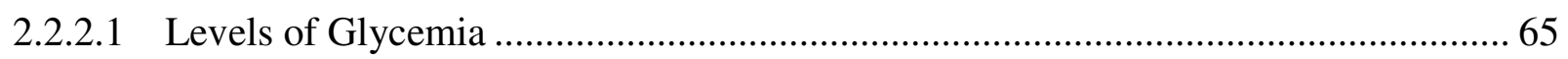

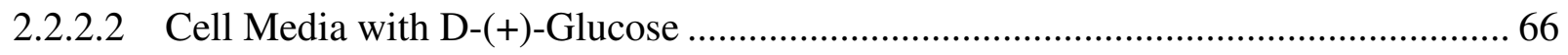

2.2.2.3 Monitoring Hyperglycemic Levels ............................................................... 68

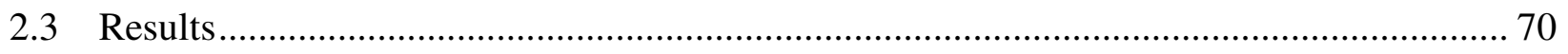

2.3.1 Results of Preliminary Testing ....................................................................... 70

2.3.1.1 Glycemic Level of Gibco Medium 199......................................................... 71

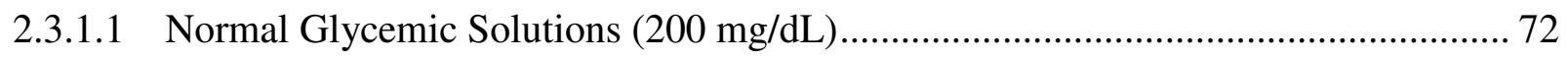

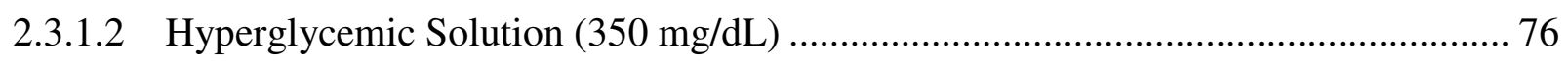

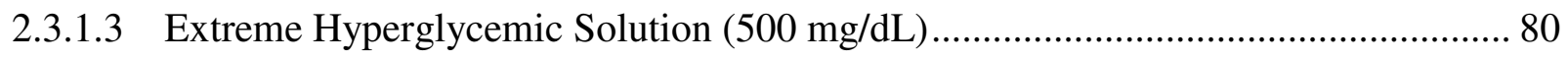

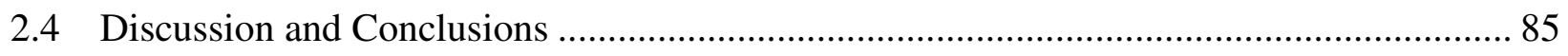

Chapter 3: Evaluation of Endothelial Cells in High Glucose Solutions.................................. 90

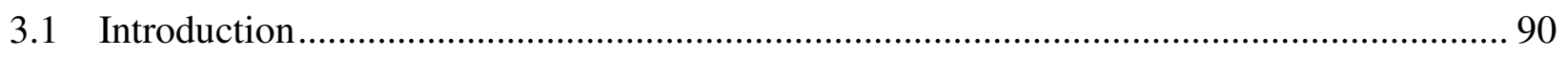

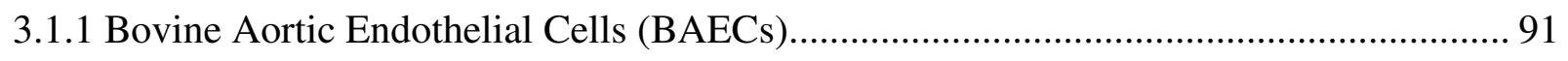


3.1.2 Human Umbilical Vein Endothelial Cells (HUVECs)

3.1.3 Purpose of This Study . 95

3.2 Methods. 96

3.2.1 Preparation of Glycemic Media Solutions 97

3.2.1.1 Preparation of the $400 \mathrm{mg} / \mathrm{dL}$ Hyperglycemic Cell Media Solution 98

3.2.2 Sterilization of the $400 \mathrm{mg} / \mathrm{dL}$ Hyperglycemic Cell Media Solutions....... 99

3.2.3 Applying the Glycemic Media Solutions to the Endothelial Cells 100

3.2.4 Evaluation of BAECs and HUVECs 103

3.3 Results. 104

3.3.1 $100 \mathrm{mg} / \mathrm{dL}$ Normal Glycemic Environment 104

3.3.1.1 Glucose Consumption 104

3.3.2 $400 \mathrm{mg} / \mathrm{dL}$ Hyperglycemic Environment. 107

3.3.2.1 Glucose Consumption 107

3.4 Investigating the Decreased Glucose Concentration at $\mathrm{t}=0$ for the $400 \mathrm{mg} / \mathrm{dL}$

hyperglycemic cell media solutions 111

3.4.1 Testing the Loss of Glucose during Syringe-Filter Sterilization 111

3.4.2 Third BAEC \& HUVEC Experiment. 114

3.4.2.1 Preparation and Application of Glycemic Cell Media Solutions 114 
3.5.1.2 Glucose Consumption ............................................................................. 115

3.5.1.3 Cell Confluency.......................................................................................... 118

3.5.2 $400 \mathrm{mg} / \mathrm{dL}$ Hyperglycemic Environment....................................................... 120

3.5.2.1 Glucose Consumption .......................................................................... 120

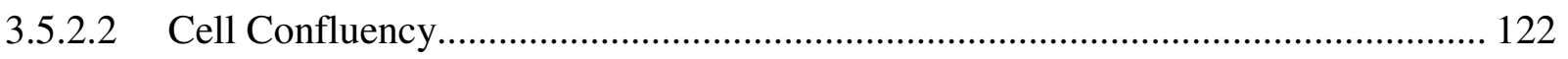

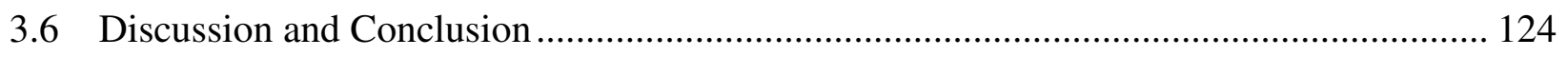

3.6.1 $100 \mathrm{mg} / \mathrm{dL}$ Normal Glycemic Environment .................................................... 125

3.6.2 $400 \mathrm{mg} / \mathrm{dL}$ Hyperglycemic Environment........................................................ 125

3.6.3 Removal Complete Cell Media during Application of Glycemic Cell Media

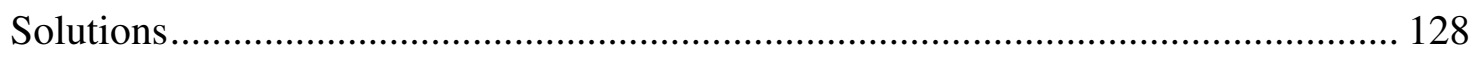

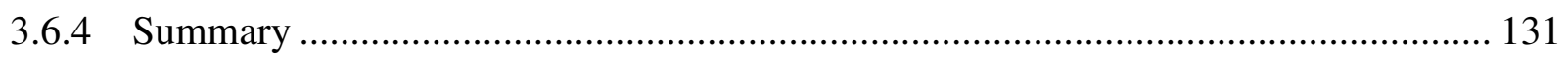

Chapter 4: Discussion \& Conclusion.......................................................................................... 134

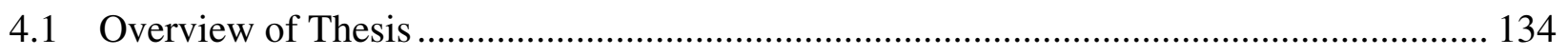

4.1.1 Diabetes Mellitus.............................................................................................. 134

4.1.2 Complications of Diabetes Mellitus ........................................................... 135

4.1.3 Purpose \& Direction of Study ................................................................. 136 
4.1.4 Method of Creating \& Monitoring Glycemic Cell Media Solutions

4.1.5 Monitoring Glucose Consumption of BAECs and HUVECs

4.2 Discussion of Thesis

4.2.1 Improvements to Test Protocols

4.2.2 Future Work in Support of this Thesis

List of References

Appendix A: Results for Preliminary Testing to Establish Method of Developing Glycemic

Cell Media Solutions.

Appendix B: Results for Experimentation to Determine Most Feasible Method of Measuring

Glucose Concentration of Developed Glycemic Cell Media Solutions. 167

Appendix C: Results for Experimentation of BAECs \& HUVECs Exposed to $100 \mathrm{mg} / \mathrm{dL}$

Normal Glycemic Cell Media Solutions \& 400 mg/dL Hyperglycemic Cell Media

Solutions 171

Appendix D: Protocol for the Development of Sterile $400 \mathrm{mg} / \mathrm{dL}$ Hyperglycemic Cell

Media Solution. 178

Appendix E: Protocol Using Aviva Accu-Chek Blood Glucose Meter. 180 


\section{List of Figures}

Figure 1 - Oscar Minkowski surgically removed the pancreas of a dog which he later realized induced diabetes mellitus. (Permission to reprint from Fischer I: Biographisches Lexikon der hervorragenden Arzte der letzten funfzig Jahre. T. 1. Monachium-Berlin: Urban \&

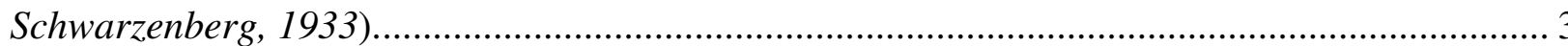

Figure 2 - Dr. Frederick Banting (shown), and medical student Charles Best, were able to extract the first pure insulin substance (Permission to reprint from Nobel Lectures, Physiology or Medicine 1922-1941, Elsevier Publishing Company, Amsterdam, 1965).

Figure 3 - Dr. John James Macleod, Professor of Physiology at the University of Toronto, funded the experimentation for the first stable extraction of insulin (Permission to reprint from Nobel Lectures, Physiology or Medicine 1922-1941, Elsevier Publishing Company, Amsterdam, 1965).

Figure 4 - James Bertram Collip, biochemist from the University of Toronto, was able to purify and stabilize the insulin extraction for human testing (Permission to reprint from the Library of the University of Toronto, Canada).

Figure 5 - Development of type I diabetes (Permission to reprint from [7]).

Figure 6 - The main contributors to the pathogenesis of type II diabetes insulin resistance are genetics, environmental factors, and the adverse effects of obesity (Permission to reprint from [19]). 
Figure 7 - Coronary artery disease results when a normal healthy coronary vessel, (a) narrows due to atherosclerosis and plaque build-up, and (b) leads to inadequate blood flow through the vessel (Permission granted from the National Heart, Lung, and Blood Institue as a part of the National institute f Health and the US Department of Health and Human Services). 20

Figure 8 - With the build-up of plaque in a coronary artery, coronary angioplasty is a medical procedure where, (a) a balloon catheter is inserted to the site of plaque buildup, (b) the balloon is inflated and pushes the plaque against the blood vessel wall, and (c) proper blood flow is restored to the artery (Permission granted from the National Heart, Lung, and Blood Institue as a part of the National institute f Health and the US Department of Health and Human Services).

Figure 9 - The graph above displays that angiographic restenosis (50\% diameter stenosis) was significantly greater in diabetic patients than non-diabetic patients (Permission to reprint by [63]).

Figure 10 - The diagram shows the progression of in-stent restenosis as a vessel injury response resulting in the proliferation of smooth muscle cells, the expression of vessel adhesion molecules, and the adhesion of leukocytes (Permission to reprint from [71]).

Figure 11 - Increased thrombus was located in the surrounding region of the stent strut (green arrows) of rats with diabetes vs. rats without diabetes (Permission to reprint from [162]). 29

Figure 12 - Long-term type I diabetes in BBDP rats is associated with enhanced in-stent restenosis four weeks after stenting the abdominal aorta (Permission to reprint by [166])......... 30 
Figure 13 - A study by Algenstaedt et al. showed that diabetic mice experienced a decrease in vascular density, but an increase is vascular diameter with an increase in glycemic concentration (Permission to reprint from [90]).

Figure 14 - Potential mechanisms by which hyperglycemia-induced mitochondrial superoxide overproduction activates four pathways of hyperglycemic damage (Permission to reprint by [167]).

Figure 15 - Glucose is an aldohexose; a hexose with an aldehyde attached at the end (Permission to reprint from [102]). 45

Figure 16 - An aldehyde is an organic compound containing a terminal carbonyl group (Permission to reprint by [103]). 46

Figure 17 - The cyclic form of glucose, glucopyranose, results from the aldehyde carbon atom double-bonded to the hydroxyl group on the fifth carbon of the open chain (Permission to reprint by [102]). 46

Figure 18 - D-glucose and L-glucose are two stereoisomers of the aldohexose sugars. However, D-glucose is the biologically active form (Permission to reprint by [102]).

Figure 19 - A glucose tolerance test illustrates that a normal patient who has consumed glucose will return to homeostasis after about 2 hours. A diabetic patient, however, will take 4-6 hours and sometimes not even reach normal glycemic levels. Distinguishing between type I and type II diabetes comes down to analyzing the plasma insulin levels - low levels in type I diabetics, and high levels in type II diabetics (Permission to reprint by [107]). 
Figure 20 - The binding of insulin to its cell membrane receptor induces a series of subsequent events, which cause the facilitated diffusion of glucose into the cell (Permission to reprint from [107]).

Figure 21 - As blood glucose concentration rises, the rate of insulin secretion rises at a rapid rate. This increase in insulin secretion is non-existent in type I diabetics, and ineffective in type II diabetics - thus, resulting in hyperglycemia (Permission to reprint by [107]).

Figure 22 - The influx of glucose into the pancreatic $\beta$-cells is controlled by glucose transporters (GLUT-2 and GLUT-4) depending on the blood glucose concentration (Permission to reprint from [107].

Figure 23 - The Aviva Accu-Chek blood glucose meter is the most popular meter used among diabetics to test blood sugar levels (Permission to reprint by Accu-Chek Blood Glucose Monitoring Systems).

Figure 24 - The Clarke Error Grid Analysis (EGA) was developed to quantify the clinical accuracy of a patient's estimate of their blood glucose compared to the value obtained on the meter. Patients could estimate their blood glucose levels by another blood glucose meter or visual test strips (Permission to reprint by [127]).

Figure 25 - The MiniMed Paradigm REAL-Time System uses a glucose sensor attached to a wireless transmitter that records blood glucose levels and graphs it on the incorporated insulin pump (Permission to reprint by Medtronic Diabetes).

Figure 26 - Sigma G6512 D-(+)-Glucose was added to Gibco Medium 199 in the attempt to create glycemic cell media solutions. 
Figure 27 - Gibco Medium 199 by Invitrogen was used as the base cell media for the experimentation of different glycemic cell media solutions. This media was also used for later experimentation when developing glycemic cell media solutions for use with human umbilical vein endothelial cells (HUVECs) 67

Figure 28 - Glucoflex-R Blood Glucose Strips by Ambe Medical Group in England (Permission to reprint by Ambe Medical Group

Figure 29 - The Invitrogen website confirmed that the composition of the Gibco Medium 199 contained $1000 \mathrm{mg} / \mathrm{L}(100 \mathrm{mg} / \mathrm{dL})$ of D-glucose. 71

Figure 30 - The plot shows that the accumulated glucose concentrations by the Aviva AccuChek blood glucose meter matched the expected glucose concentration of $200 \mathrm{mg} / \mathrm{dL}$ for each solution. The glucose concentration rose rapidly, but plateaued at the expected value after roughly 2 hours of mixing. 73

Figure 31 - The recorded glucose levels, using the Glucoflex-R visual test strips, were within the expected range of $200 \mathrm{mg} / \mathrm{dL}$. However, the variance of the recorded results was quite high, and is not an ideal characteristic for a sensitive hyperglycemic environment. 74

Figure 32 - The plot shows that the glucose concentrations recorded by the Aviva Accu-Chek blood glucose meter were similar to the expected glucose concentration of $350 \mathrm{mg} / \mathrm{dL}$ for each solution. The glucose concentration rose rapidly, but settled after approximately four hours of mixing. 
Figure 33 - The recorded glucose concentrations, using the Glucoflex-R visual test strips, for the $350 \mathrm{mg} / \mathrm{dL}$ solutions, were within range of the expected glucose concentration. However, the extremely high variance further proved the lack of precision of the visual test strips. 78

Figure 34 - Even though the Accu-Chek glucose meter continued to measure glucose concentrations higher than the expected value of $400 \mathrm{mg} / \mathrm{dL}$, the average measurements were much more accurate compared to the average measurements for the other glycemic environments 82

Figure 35 - The Glucoflex-R strips were able to monitor the glucose concentrations of the extremely hyperglycemic $400 \mathrm{mg} / \mathrm{dL}$ solutions much more accurately than the other glycemic environments. 83

Figure 36 - This study showed how exposure of BAECs to a hyperglycemic environment hindered nitric oxide (eNOS) activity. BAECs exposed to a $5 \mathrm{mM}(90 \mathrm{mg} / \mathrm{dL})$ normal glycemic environment had higher eNOS activity than BAECs exposed to the $30 \mathrm{mM}(540 \mathrm{mg} / \mathrm{dL})$ hyperglycemic environment. eNOS plays an important role in preventing many vascular diseases (permission to reprint from [141]).

Figure 37 - TUNEL staining will typically show apoptotic configuration among the HUVECs with shrinkage and fragmentation of the nuclei. This figure shows how exposure of HUVECs in a $30 \mathrm{mM}$ hyperglycemic environment caused a significant amount of cell death after 72 hours compared to after only 24 hours. Supporting pictures are also shown, which support greater apoptosis in the $30 \mathrm{mM}$ hyperglycemic environment (permission to reprint from [145]).

Figure 38 - For experiments with BAECs, the glycemic cell media solutions were made using Lonza Endothelial Cell Basal Medium (EBM). 
Figure 39 - After adding the Sigma G6152 D-(+)-Glucose to the cell media, the solution was stirred with a magnetic stir plate and bar for at least six hours at $25^{\circ} \mathrm{C}$ to ensure uniform mixing.

Figure 40 - Syringe-filter sterilization was conducted to sterilize the $400 \mathrm{mg} / \mathrm{dL}$ hyperglycemic cell media solutions. (a) $10 \mathrm{~mL}$ of the solution was taken up by a $10 \mathrm{~mL}$ syringe and the 0.22 $\mu \mathrm{m}$ syringe filter was added onto the end of the syringe. (b) The $10 \mathrm{~mL}$ of solution was slowly transferred into a sterile $50 \mathrm{~mL}$ conical. This entire sterilization was conducted in a laminar flow hood. 100

Figure 41 - To introduce the glycemic cell media solutions to the endothelial cells, an electronic pipette was used. The $100 \mathrm{mg} / \mathrm{dL}$ solution was added to the bottom three wells, and the 400 $\mathrm{mg} / \mathrm{dL}$ solution was added to the top three wells. 101

Figure 42 - (a) For the first BAEC experiment, $10 \mathrm{~mL}$ of the glycemic cell media solutions was added to each well. The $400 \mathrm{mg} / \mathrm{dL}$ cell media was introduced to the cells in the top three wells, and the $100 \mathrm{mg} / \mathrm{dL}$ cell media was introduced to the cells in the bottom three wells. (b) For the second BAEC experiment and the first two HUVEC experiments, the volume of media introduced to each well was reduced to $3 \mathrm{~mL}$. Again, the $400 \mathrm{mg} / \mathrm{dL}$ cell media was introduced to the cells in top three wells, and $100 \mathrm{mg} / \mathrm{dL}$ cell media was introduced to the cells in the bottom three wells. 102

Figure 43 - There was minimal decrease in glucose concentration throughout the 72 hour test period; therefore, the glucose consumption for the BAEC and HUVEC studies was minimal.. 105 
Figure 44 - There was a greater decrease in glucose concentration throughout the 72 hour test period. The data also shows that there was a substantial decrease in glucose concentration for the second BAEC experiment, as well as the two HUVEC experiments starting at $\mathrm{t}=0$. 108

Figure 45- Amount of glycemic cell media solution added to each well for the third BAEC \& HUVEC experiments

Figure 46 - The BAECs and HUVECs exposed to the $100 \mathrm{mg} / \mathrm{dL}$ normal glycemic cell media solutions in the third experiment exhibited similar decrease in glucose concentration. 116

Figure 47 - (a) The cell confluency of the HUVECs exposed to the $100 \mathrm{mg} / \mathrm{dL}$ normal glycemic cell media solution did not change from $\mathrm{t}=0$ to $\mathrm{t}=72$ hours. (b) Additionally, the cell confluency of the BAECs exposed to the $100 \mathrm{mg} / \mathrm{dL}$ normal glycemic cell media solution did not change from $t=0$ to $t=72$ hours either.

Figure 48 - The BAECs and HUVECs exposed to the $400 \mathrm{mg} / \mathrm{dL}$ hyperglycemic cell media solutions for the third experiment exhibited a decrease in glucose concentration over the 72 hour test time; thus, exhibiting glucose consumption.

Figure 49 - The cell confluency of the HUVECs exposed to the $400 \mathrm{mg} / \mathrm{dL}$ hyperglycemic cell media solution did not significantly decrease from $t=0$ to $t=72$ hours. (b) Additionally, the cell confluency of the BAECs exposed to the $400 \mathrm{mg} / \mathrm{dL}$ hyperglycemic cell media solution did not decrease from $t=0$ to $t=72$ hour. 123 


\section{List of Tables}

Table 1 - Most significant environmental factors that contribute to the increased incidence of

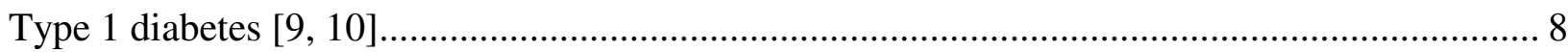

Table 2 - Common complications associated with diabetes mellitus resulting from hyperglycemia

Table 3 - Amount of Sigma G6152 D-(+)-Glucose added to $100 \mathrm{~mL}$ of Gibco Medium 199 for

200 mg/dL Normal Glycemic Solutions

Table 4 - Average Glucose Concentrations Recorded with Accu-Chek Blood Glucose Meter \&

Glucoflex-R Visual Blood Glucose Strips for 200 mg/dL Glycemic Solutions 75

Table 5 - Amount of Sigma G6152 D-(+)-Glucose added to $100 \mathrm{~mL}$ of Gibco Medium 199 for the $350 \mathrm{mg} / \mathrm{dL}$ hyperglycemic solutions

Table 6 - Average Glucose Concentrations Recorded with Accu-Chek Blood Glucose Meter \& Glucoflex-R Visual Blood Glucose Strips for 350 mg/dL Hyperglycemic Solutions. 79

Table 7 - Amount of Sigma G6152 D-(+)-Glucose added to 100 mL of Gibco Medium 199 for $500 \mathrm{mg} / \mathrm{dL}$ extreme hyperglycemic solutions.....

Table 8 - Average Glucose Concentrations Recorded with Accu-Chek Blood Glucose Meter \& Glucoflex-R Visual Blood Glucose Strips for 500 mg/dL extreme hyperglycemic solutions ..... 84

Table 9 - Recipes to Create the 400 mg/dL Hyperglycemic Cell Media Solutions for Initial BAEC \& HUVEC Experiments. 
Table 10 - Average Glucose Concentrations of 100 mg/dL Normal Glycemic Cell Media

Solutions Exposed to BAECs \& HUVECs ................................................................... 105

Table 11 - Two-Tailed T-Tests Results for BAECs \& HUVECs Exposed to $100 \mathrm{mg} / \mathrm{dL}$ Normal

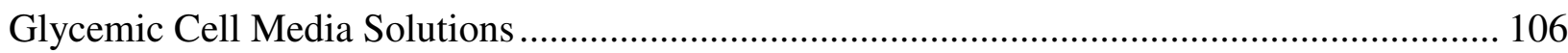

Table 12 - Average Glucose Concentrations of 400 mg/dL Hyperglycemic Cell Media Solutions

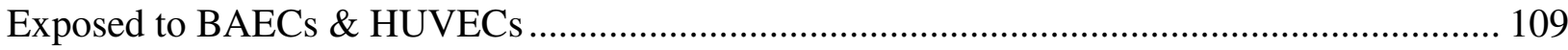

Table 13 - Two-Tailed T-Tests Results for BAECs \& HUVECs Exposed to $400 \mathrm{mg} / \mathrm{dL}$

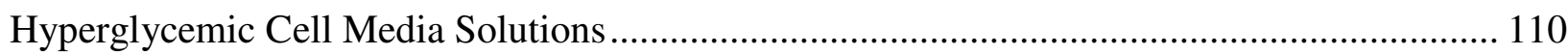

Table 14 - Recipes to Create 400 mg/dL Hyperglycemic Solutions to Test Glucose Loss during

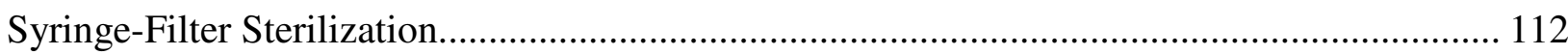

Table 15 - Effect of Syringe-Filter Sterilization on 400 mg/dL Hyperglycemic Cell Media

Solutions

Table 16 - Recipes to Create 400 mg/dL Hyperglycemic Solutions for Final BAEC \& HUVEC

Experiments 114

Table 17 - Average Glucose Concentrations of the 100 mg/dL Normal Glycemic Cell Media

Solutions Exposed to BAECs \& HUVECs of Final Experiments 116

Table 18 - Two-Tailed T-Test Results for the Final BAEC \& HUVEC Experiments Exposed to 100 mg/dL Normal Glycemic Cell Media Solutions 117

Table 19 - Average Glucose Concentrations of the 400 mg/dL Hyperglycemic Cell Media Solutions Exposed to BAECs \& HUVECs of Third Experiments 121 
Table 20 - Two-Tailed T-Tests for Final BAEC \& HUVEC Experiments Exposed to the 400 mg/dL Hyperglycemic Cell Media Solutions ...................................................................... 122

Table 21 - Two-Tailed T-Test Results for First BAEC Experiment Exposed to $400 \mathrm{mg} / \mathrm{dL}$ Hyperglycemic Cell Media Solutions

Table 22 - Calculated Glucose Concentrations Compensating for Additional $2 \mathrm{~mL}$ of Complete Cell Media that was mistakenly not removed 130

Table 23 - Summary of Conclusions from Experimentation with BAECs \& HUVECs in 100 $\mathrm{mg} / \mathrm{dL} \& 400 \mathrm{mg} / \mathrm{dL}$ Environments

Table 24 - Summary of Experimentation, Results, and Conclusions of Entire Thesis 140

Table 25 - Development of $100 \mathrm{mg} / \mathrm{dL}$ Solution Trial \#1 163

Table 26 - Development of $100 \mathrm{mg} / \mathrm{dL} \& 180 \mathrm{mg} / \mathrm{dL}$ Solutions Trial \#2 164

Table 27 - Development of 100 mg/dL Normal Glycemic \& 400 mg/dL Hyperglycemic

Solutions Trial \#3. 165

Table 28 - Development of 100 mg/dL Normal Glycemic \& 350 mg/dL Hyperglycemic Solutions Trial \#4. 166

Table 29 - Method of Monitoring 200 mg/dL Normal Glycemic Environment Test Results .... 168

Table 30 - Method of Monitoring 350 mg/dL Hyperglycemic Environment Test Results 169

Table 31 - Method of Monitoring 500 mg/dL Hyperglycemic Environment Test Results 170

Table 32 - BAEC Glucose Consumption Comparative Study Experiment \#1 172 xxiii 
Table 33 - BAEC Glucose Consumption Comparative Study Experiment \#1 - Well $\Delta$ mg/dL

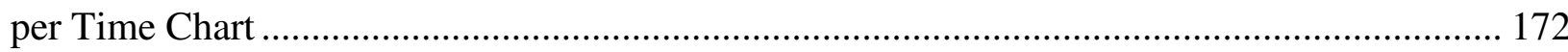

Table 34 - HUVEC Glucose Consumption Comparative Study Experiment \#1 ...................... 173

Table 35 - HUVEC Glucose Consumption Comparative Experiment Trial \#1 - Well $\Delta$ mg/dL

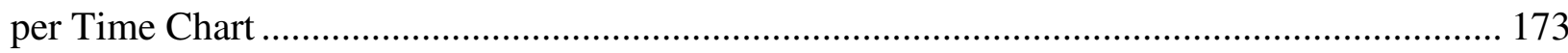

Table 36 - BAEC Glucose Consumption Comparative Study Experiment \#2 ........................ 174

Table 37 - BAEC Glucose Consumption Comparative Study Experiment \#2 - Well $\Delta$ mg/dL

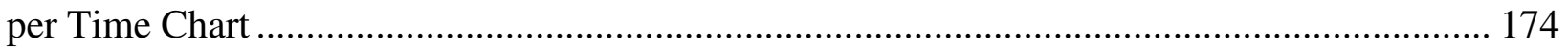

Table 38 - HUVEC Glucose Consumption Comparative Study Experiment \#2 ...................... 175

Table 39 - HUVEC Glucose Consumption Comparative Study Experiment \#2 - Well $\Delta$ mg/dL

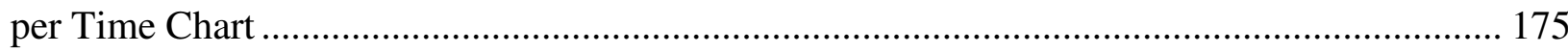

Table 40 - BAEC Glucose Consumption Comparative Study Experiment \#3 ......................... 176

Table 41 - BAEC Glucose Consumption Comparative Study Experiment \#3 - Well $\Delta$ mg/dL

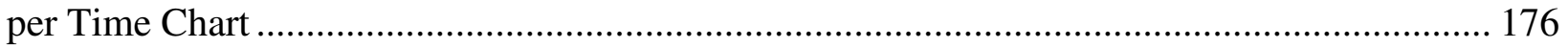

Table 42 - HUVEC Glucose Consumption Comparative Study Experiment \#3 ...................... 177

Table 43 - HUVEC Glucose Consumption Comparative Study Experiment \#3 - Well $\Delta$ mg/dL

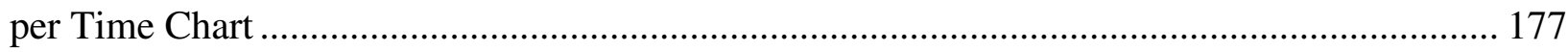




\section{Chapter 1: An Introduction to Diabetes Mellitus}

\subsection{The History of Diabetes Mellitus}

The name Diabetes Mellitus was coined in the 1600's by scientists who were describing their observations of patients with a particular set of symptoms; "diabetes" - intense thirst, profuse urination, rapid waste production, intense vomiting, drowsiness eventually leading to a coma and then death; "mellitus" - the apparent sweetness of the patient's urine [1]. The outcome of diabetes was a slow and painful death. There was no known reason behind the cause of the disease and no thought of what a possible cure could be. This is why the discovery of insulin in 1921 was one of the most dramatic discoveries in the history of diabetes and medicine, contributing to a drastic change in lifestyle for patients with the disease.

Oxford physician Thomas Willis took the first step towards better understanding diabetes in the $17^{\text {th }}$ century [1]. He noted the sweet taste of urine from diabetic patients along with the high volume they were producing. He was also the first to distinguish the sweet taste of urine from patients with Diabetes Mellitus versus the non-sweet urine of patients with Diabetes Insipidis [1]. While others were certain that diabetes was due to renal complications, Thomas Willis believed that complications in blood were the main contributor to the disease. In 1776, Willis' theory was supported by scientist Matthew Dobson, of Liverpool, as Dobson discovered that the sweet taste of urine was caused by a high concentration of glucose. He proved that there was an excess of glucose in the blood of those who produced such sweet tasting urine [2].

Claude Bernard, of France, was one of the greatest figures of diabetes in the first half of the $19^{\text {th }}$ century. Some of his greatest findings included the discovery of the liver's ability to 
store glycogen, as well as the liver's ability to convert glycogen and secrete sugar into the blood. This led him to believe that diabetes was due to an over-secretion of sugar from the liver [1]. Claude Bernard was such an influential figure that his view on the cause of diabetes was a staple in the medical society. However, by extraordinary strokes of chance, an alternative proposition regarding the cause and possible control of diabetes was discovered.

\subsubsection{The Discovery of Insulin}

In 1879, Joseph Von Mering of Germany discovered that removing the pancreas caused diabetes. Interested mostly in digestion, Von Mering wanted to study the digestive effects of the pancreas by removing it from a dog and analyzing what digestive limitations the dog exhibited. Von Mering's colleague, Oscar Minkowski (Figure 1) performed the procedure and later discovered that the dog had urinated all over its cage even though it had been house trained. Much more interested in what this discovery might mean, Minkowski tested the urine and found high levels of glucose; thus, implying that the pancreas contained the anti-diabetic agent that controlled glucose level and could possibly cure diabetes [1]. Unfortunately, the extraction of this anti-diabetic substance was extremely difficult. It was widely held that the substance was in the pancreatic islets, but it was believed that digestive enzymes produced by the pancreas were interfering with the extraction. While some scientists were able to develop an unstable extract that was lowering the blood glucose levels of dogs, none were successful in purifying the substance for human testing [2]. Though discouraging, there was no lack of appreciation of how much potential the purification of the anti-diabetic substance could have on the advancement of diabetes treatment. 


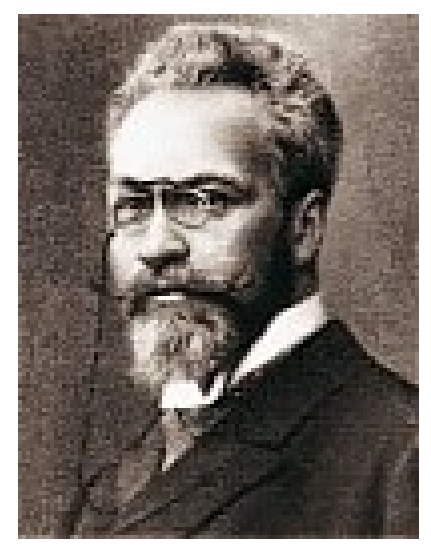

Figure 1 - Oscar Minkowski surgically removed the pancreas of a dog which he later realized induced diabetes mellitus. (Permission to reprint from Fischer I: Biographisches Lexikon der hervorragenden Arzte der letzten funfzig Jahre. T. 1. Monachium-Berlin: Urban \& Schwarzenberg, 1933).

Finally in 1921, one of the greatest medical discoveries occurred at the University of Toronto, Canada. Dr. Fredrick Banting (Figure 2), an unsuccessful orthopedic surgeon, was reading on the association of pancreatic dysfunction with diabetes and became convinced that he could produce the anti-diabetic substance [3]. Ironically, his greatest strength was his ignorance towards how difficult the procedure was since he was unaware that so many other scientists had attempted to find it in the forty years since Minkowski's discovery. Nonetheless, Banting approached Dr. John James Richard Macleod (Figure 3), Professor of Physiology at the University of Toronto, and requested the funds and facilities to perform the experiment. Knowing the difficulties behind the procedure, Macleod assigned medical student Charles Best to aid Banting in the assembly of the experiment. Banting originally believed that the external secretion of the pancreas was destroying the "insulin;" thus, he tied off the pancreatic duct and allowed for pancreas atrophy [3]. Unfortunately, there was difficulty in stabilizing the pancreatic secretion, so Macleod assigned James Bertram Collip (Figure 4), biochemist at the University of Toronto, to perform standard chemical purification in order to stabilize the substance for human 
testing. Within six weeks, Collip felt comfortable enough with the insulin he had isolated to try it on a human for the first time [3].

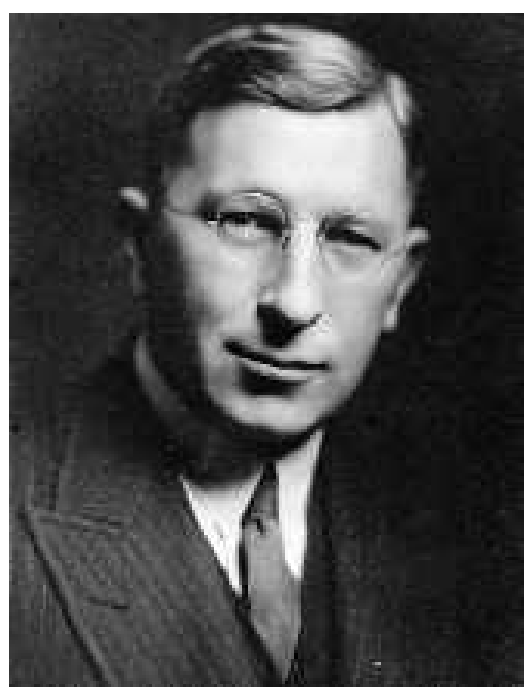

Figure 2 - Dr. Frederick Banting (shown), and medical student Charles Best, were able to extract the first pure insulin substance (Permission to reprint from Nobel Lectures, Physiology or Medicine 1922-1941, Elsevier Publishing Company, Amsterdam, 1965).

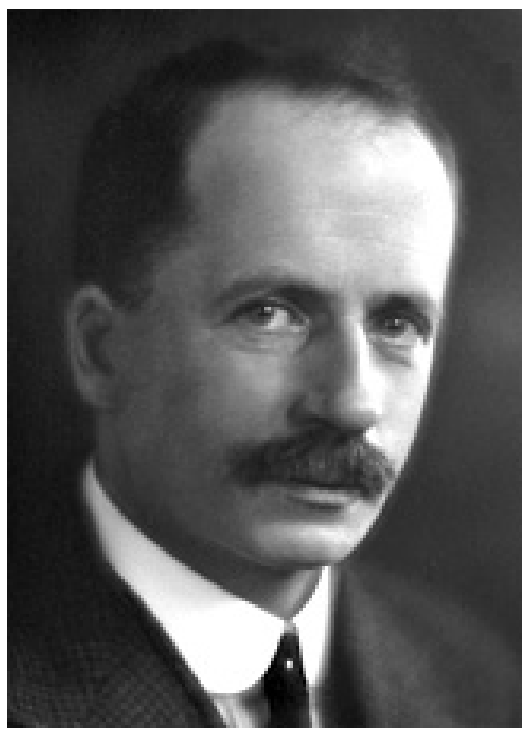

Figure 3 - Dr. John James Macleod, Professor of Physiology at the University of Toronto, funded the experimentation for the first stable extraction of insulin (Permission to reprint from Nobel Lectures, Physiology or Medicine 1922-1941, Elsevier Publishing Company, Amsterdam, 1965). 


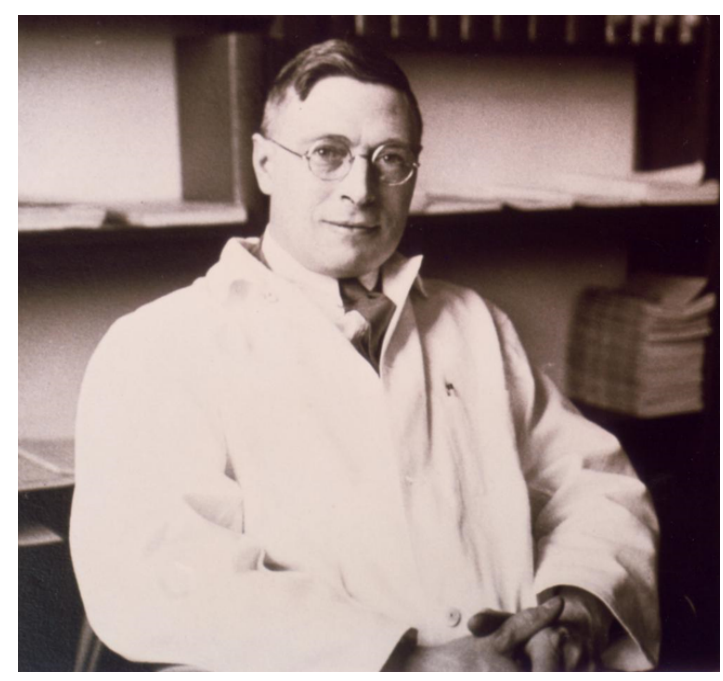

Figure 4 - James Bertram Collip, biochemist from the University of Toronto, was able to purify and stabilize the insulin extraction for human testing (Permission to reprint from the Library of the University of Toronto, Canada).

The first human trial was performed on a young 14 year-old boy dying of diabetes. To everyone's astonishment, the insulin injection lowered the boy's blood sugar, rid the glucose in the urine, and eventually helped him regain his body weight and strength [3]. It was a miraculous transformation; a universally fatal disease had been cured - or if not cured, at least controlled. Banting and Best published their first paper in 1922, and in 1923 Banting and Macleod were awarded the Nobel Prize in Medicine for their remarkable discovery [3].

\subsubsection{Impact of the Discovery of Insulin}

Before the discovery of insulin, diabetes was a slow and heartbreaking death. Mostly diagnosed in young children, the only method of treatment was a strict diet that eventually equaled starvation. It was an unfortunate situation for early "diabetologists," especially Dr. Frederick Allen, of New York, who had nothing else to offer other than starvation [1]. If the patient got an infection and the diabetes got worse, the answer was even stricter starvation - a miserable situation for the young patients, as well as the parents. However, the arrival of insulin 
brought a revival to the field that was best expressed by a nurse at Dr. Allen's psychiatric hospital in New York. She wrote [3]:

“...the mere illusion of new hope cajoled patient after patient into new life. Diabetics who had not been out of bed for weeks began to trail weekly about, clinging to walls and furniture. Big stomachs, skin-and-bone necks, skull-like faces, feeble movements, all ages, both sexes - they looked like an old Flemish painter's depiction of a resurrection after famine. It was a resurrection, a crawling stirring, as of some vague springtime."

The patients heard that Dr. Allen had come back from Toronto:

"Bed immediately after dinner was the rule of our patients. But not that evening. My office opened on the big center hallways. I could see them drifting in, silent as the bloated ghosts they looked like. Even to look at one another would have painfully betrayed some of the intolerable hope that had brought them. So they just sat and waited, eyes on the ground.

It was growing dark outside. Nobody had yet seen Doctor Allen. His first appearance would be at his dinner, which followed the patients' dinner hour. We all heard his step coming along the covered walk, past the entrance to the main hallways. His wife was with him, her quick tapping pace making a queer rhythm with his. The patients' silence concentrated on that sound. When he appeared through the open doorway, he caught a full beseeching of a hundred pair of eyes. It stopped him dead. Even now I am sure it was minutes before he spoke to them, his voice curiously mingling concern for his patients with an excitement that he tried his best not to betray. 'I think,' he said, 'I think we have something for you' '”

The discovery of insulin changed the lives of doctors and patients. Those who were headed to certain death in the coming years lived on for another fifty. The first insulin treatment was quick acting insulin that required two injections a day - long lasting insulin was still to come [4]. What was significant about this discovery was that it contradicted Claude Bernard's work. It was not an over-secretion of glucose from the liver that was causing diabetes, but rather an autoimmune-induced deficiency of islet $\beta$-cells in the pancreas - cells that produced and secreted 
insulin [1]. This new understanding of diabetes brought about the distinction of two different types of the disease - insulin dependent and non-insulin dependent diabetes mellitus. In 1936, Harold Himsworth demonstrated the important biochemical differences in the two types by identifying insulin sensitivity in type I and insulin resistance in type II diabetes [2]. This brought about a new perspective in the search for the cure of diabetes - there was no longer one single cure for the disease.

\subsection{Overview of Diabetes Mellitus}

Diabetes Mellitus is a disease that causes the body to insufficiently produce or improperly use insulin. Insulin is a pancreatic hormone, secreted from $\beta$-cells in the pancreatic islets, that breaks down sugars, starch, etc. into energy for the body. Diabetes Mellitus comes in two common forms - insulin deficient, or type I diabetes, and insulin resistant, or type II diabetes. Type I diabetes, also known as juvenile diabetes, is most common in children, while type II diabetes is diagnosed mostly in adults. Diabetes Mellitus is also one of the most prevalent diseases diagnosed in America today. According the American Diabetes Association, about 23.6 million children and adults in the United States of America have diabetes mellitus (about $7.8 \%$ of the population), of which only 17.9 million are diagnosed. This means that nearly a quarter of the 23.6 million children and adults do not know that they have diabetes. Out of the 23.6 million with diabetes, about $10 \%$ have type I, leaving the other $90 \%$ diagnosed with type II diabetes. This section will discuss the key similarities and differences between the two types of the disease. 


\subsubsection{Type I (Insulin Deficient) Diabetes Mellitus}

\subsubsection{Causes/Pathology}

Insulin deficient ( also known as insulin dependent) diabetes mellitus (IDDM), or type I diabetes mellitus, is a T-cell mediated autoimmune disease resulting in the selective destruction of insulin-secreting pancreatic $\beta$-cells through the detrimental immunoregulation of inflammatory cytokines and auto-aggressive T lymphocytes [5,6]. While the etiology of $\beta$-cell destruction is unknown, researchers are proposing that genetically susceptible persons, who are exposed to certain environmental agents, trigger the inflammatory autoimmune response [7]. Various environmental agents have been proposed to contribute to the pre-clinical onset of the disease, as outlined in Table 1, but no specific environmental factors have been identified with certainty [8].

Table 1 - Most significant environmental factors that contribute to the increased incidence of Type 1 diabetes $[9,10]$.

\begin{tabular}{|cl|}
\hline Environmental Factors & \multicolumn{1}{c|}{ Hypothesis } \\
\hline Viral infections & Viral hypothesis \\
\hline Exposure to certain food constituents & Dietary hypothesis \\
\hline $\begin{array}{c}\text { Increased hygiene; lack of childhood } \\
\text { infections }\end{array}$ & Hygiene hypothesis \\
\hline Rapid growth in early childhood & Accelerator hypothesis \\
\hline
\end{tabular}

As discussed before, type I diabetes is most common in children and young adults.

Unfortunately, the incidence of type I diabetes seems to be increasing 3-5\% per year [11].

There are five stages in the development of type I diabetes (Figure 5) [6, 7]. 
1. Genetic susceptibility;

2. Pre-clinical $\beta$-cell autoimmunity with gradual dysfunction of insulin secretion;

3. Onset of clinical diabetes;

4. Transient clinical remission; and

5. Established diabetes associated with acute and chronic complications and premature death.

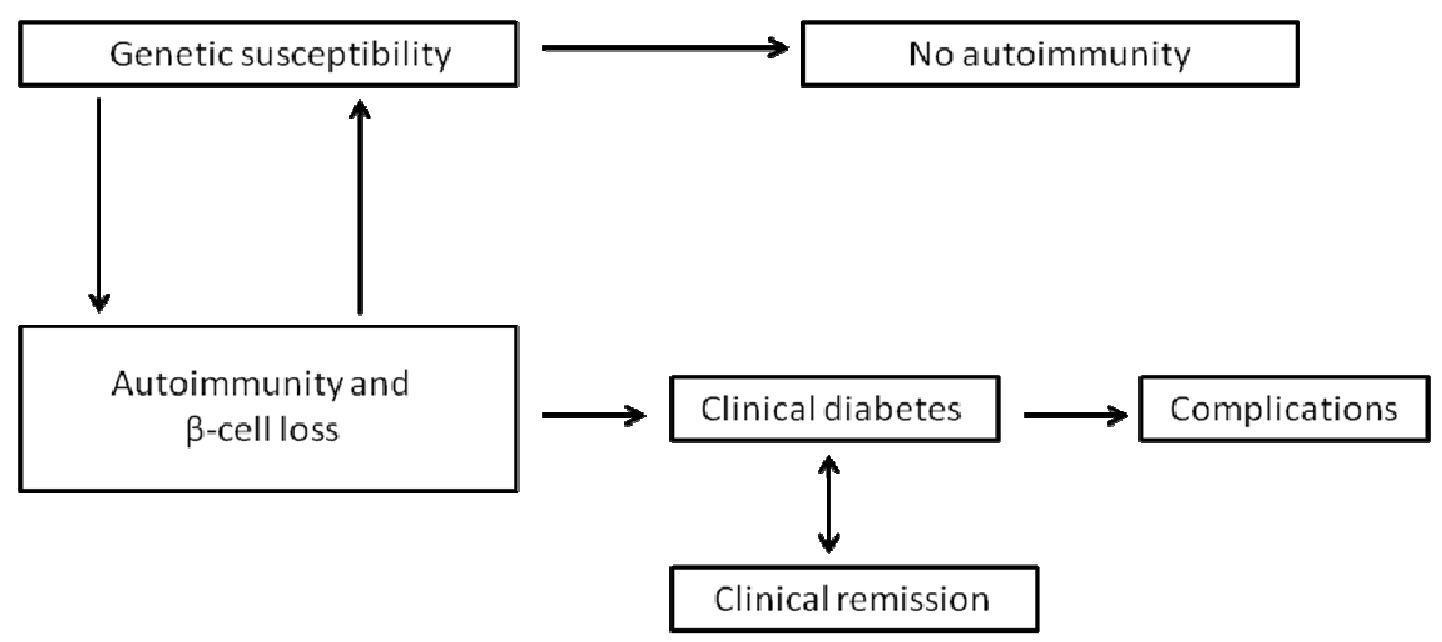

Figure 5 - Development of type I diabetes (Permission to reprint from [7]).

Environmental agents trigger the autoimmune attack against the pancreatic $\beta$-cells due to the presence of certain antibodies. These antibodies include islet cell antibodies (ICA) against islet-related antigens, anti-insulin auto-antibodes (IAA), anti-64 kDa protein antibodies, antiICA69, and anti-ICA512 [12, 13, 14, and 15]. These antibodies are now used for the detection of patients high at risk for the development of type I diabetes. Studies have found that the presence of two or more of the mentioned antibodies is highly predictive for the development of type I diabetes [16]. 
The first thing to happen during the pre-clinical onset of type I diabetes is the loss of first-phase insulin secretion. This is due to the gradual degeneration of pancreatic $\beta$-cells; however, the patient maintains a stable glycemic state [6]. The presence of these autoantibodies, with $\beta$-cell antigens, also leads the onset of hyperglycemia due to the loss of first-phase insulin secretion, and eventually results in the clinical onset of diabetes [7]. A significant effort is being made to develop antigen- and antibody-based immunotherapies that effectively prevent and/or treat type I diabetes [17]. While transient remission follows the clinical onset of the disease, the disease continues to develop resulting in further complications that greatly degenerate the rest of the body [18]. These complications will be discussed further in a subsequent section. Type I diabetic patients manage their hyperglycemia with daily injections of insulin via insulin pens or by subcutaneous insulin infusion therapy with an insulin pump. While effective, insulin therapy is by no means a cure.

\subsubsection{Type 2 (Insulin Resistant) Diabetes Mellitus}

\subsubsection{Causes/Pathology}

Insulin resistant (also known as insulin independent) diabetes mellitus, or type II diabetes mellitus, is the most common form of diabetes. Type II diabetes is characterized by the body's resistance to the metabolic effects of insulin - especially its suppressive effects on the body's blood glucose levels. Many clinical disorders, such as hypertension, dyslipidemia, and atherosclerosis, are related to the development of insulin resistance and are linked to the high glucose levels of type II diabetes. Several factors contribute to the impaired function of insulin in type II diabetes - genes, environmental factors, and obesity (Figure 6). 


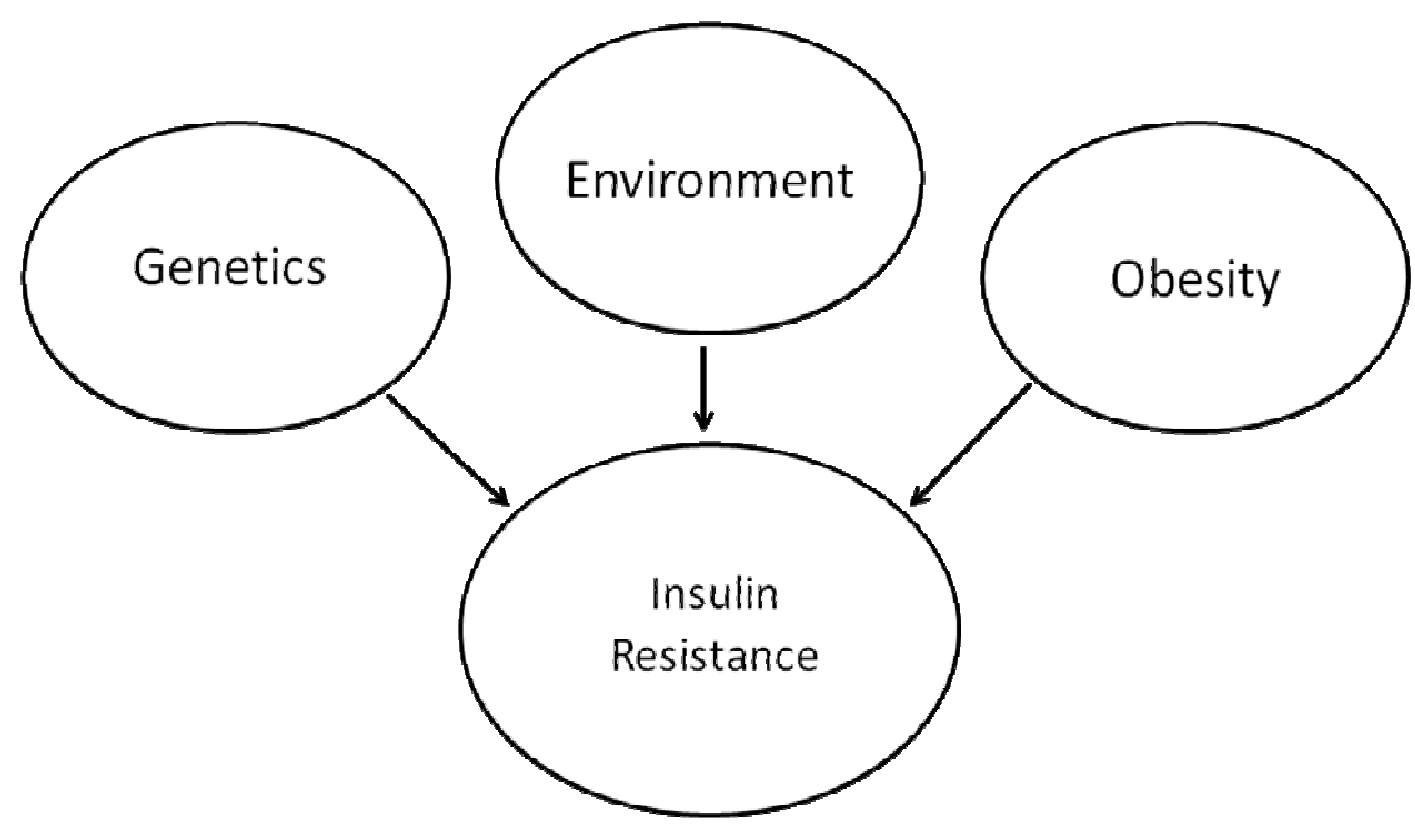

Figure 6 - The main contributors to the pathogenesis of type II diabetes insulin resistance are genetics, environmental factors, and the adverse effects of obesity (Permission to reprint from [19]).

\subsubsection{Genetics}

Type II diabetics may exhibit defects in insulin action, as well as insulin secretion; both believed to be genetically predetermined [19]. Several rare mutations in genes associated with insulin resistance have been linked to extreme insulin resistance, including mutations of insulin receptors or polymorphisms of specific proteins (i.e. G972R polymorphism of insulin receptor substrate-1). Genes associated with the development of type II diabetes include Transcription factor 7-like 2 (TCFL2), KCNJ11, just to name a few [20]. TCFL2 is a protein that acts as a transcription factor, and variance in this gene has been linked to the development of type II diabetes. KCNJ11 is a gene that encodes the Kir6.2 major subunit of the ATP-sensitive K+ channel. Mutations to this gene are linked to development of congenital hyperinsulinism, which 
can lead to insulin resistance due to its over activity. There is an extremely strong inheritance pattern for type II diabetes.

\subsubsection{Environment}

Environmental factors that lead to insulin resistance include different medical conditions, homeostatic imbalance within the body, and life style choices (exercise and diet). For example, different factors of renal failure, such as accumulation of uremic toxins, increased levels of growth hormone (described below), abnormal filtration of glucose, metabolic acidosis, and dyslipidemia are proposed mechanisms of increased insulin resistance [21]. Insulin resistance has also been identified as a result to different types of cancers, particularly pancreatic cancer [22]. Factors involving homeostatic imbalance, include abnormal hormone levels, especially excess hormone levels such as hyperinsulinism - an abnormally high level of insulin secretion a classic indicator of insulin resistance [23].

Growth hormone also acts in many levels to block insulin activity, including inhibiting the phosphorylation of the insulin receptor and its principal signaling molecule, IRS-1, in response to insulin administration [20]. This results in a reduced sensitivity of insulin in both glucose uptake and suppressing glucose production. In addition, insulin resistance induced by abnormal nutrient intake, such as a high glucose diet causing hyperglycemica (glucose toxicity) or increased levels of circulating free fatty acids, is a common feature of insulin resistance and type II diabetes [24]. Poor diet paired with poor levels of exercise have linked the onset of type II diabetes with obesity - a significant factor common among type II diabetics, especially when the disease's strong inheritance pattern was discussed. 


\subsubsection{Obesity}

Obesity is an important factor in the pathogenesis of insulin resistance and the increased risk of type II diabetes [20]. In most patients, obesity is associated with inflammation of white adipose tissue resulting from chronic activation of the innate immune system, which can lead to insulin resistance, impaired glucose tolerance and type II diabetes [25]. Although BMI accounts for only a part of the variance in insulin sensitivity, obesity and insulin resistance are related; however, insulin resistance generally is directly proportional to body-mass [26].

Adipose tissue influences insulin action in the whole body through the release of free fatty acids and adipose-derived proteins (proinflammatory peptides that cause inflammation of adipose tissue, such as adiponectin, leptin, and resistin), and has dramatic effects on glucose metabolism and insulin effectiveness [27, 28, 29]. These proinflammatory molecules induce insulin resistance and can contribute to complications in obese diabetic patients, including atherosclerosis [30]. It is still argued whether insulin resistance is the primary defect that precedes $\beta$-cell failure in the development of hyperglycemia and type II diabetes, or vice versa. However, in the end, there is a linear decrease in first-phase insulin release and insulin sensitivity in patients who are excessively in a hyperglycemic state [31]. Along with obesity, abnormal fat distribution and physical inactivity suffer the consequences of insulin resistance that include hyperglycemia, high triglycerides, hypertension, atherosclerosis (just to name a few) - all having shown to be predictors of type II diabetes [31]. 


\subsubsection{Phases of Insulin Secretion}

In non-diabetics, about $50 \%$ of the total daily insulin is secreted during basal periods (periods in between meals) to suppress lipids, proteins, and glucose. The remainder of insulin is rapidly secreted postprandial in response to a meal. This rapid release, or bolus, of insulin from the pancreatic $\beta$-cells is called the "first phase" of insulin secretion. The first phase promotes utilization of the prandial nutrients, suppresses liver glycogen-to-glucose production, and limits the elevation of blood glucose levels. First-phase insulin secretion occurs within two minutes of food ingestion and continues for approximately ten minutes. The second phase of insulin secretion follows and is sustained until normal blood glucose levels are restored. The effects of hyperglycemia on insulin secretion will be discussed further in the following chapter.

\subsection{4 $\beta$-cell Dysfunction of Diabetes Mellitus}

Pancreatic $\beta$-cell dysfunction is an early and extremely significant step in the progression of the pathophysiology of type II diabetes [32]; opposed to full degeneration of $\beta$ cells in type I diabetes. Functioning pancreatic $\beta$-cells accomplish proper regulation and storage of glucose through a negative feedback loop where elevated glycemic concentrations upregulate insulin secretion from the pancreatic $\beta$-cells, which in turn lowers glycemic concentrations by inhibiting hepatic glucose production and increasing glucose uptake by cells on target organs [33]. Assessments have shown that $\beta$-cell dysfunction affects both the firstphase and second-phase insulin response to elevated blood glucose. Thus, the decline in $\beta$-cell function precedes the development of hyperglycemia and continues after the clinical diagnosis of type II diabetes [34]. This characteristic of type II diabetes - the loss of first-phase insulin response to a meal - is the main cause of postprandial hyperglycemia seen in diabetics [33]. The 
defects of insulin resulting from type II diabetes are likely due to both the cellular dysfunction and reduction of the $\beta$-cell mass [34]. The mechanism and etiology of $\beta$-cell dysfunction is still being studied, but proposed hypotheses include glucose toxicity: chronic hyperglycemia causing hyperstimulation and overwork of the $\beta$-cells; and $\beta$-cell exhaustion: hyperstimulation of insulin secretion due to chronic hyperglycemia. The dramatic involvement of $\beta$-cell function in the development of type II diabetes will result in pancreatic $\beta$-cell-directed therapies for the treatment of the disease.

\subsubsection{Major Complications of Diabetes Mellitus}

There are numerous major complications that result from diabetes mellitus. As discussed earlier, type I diabetes is characterized by complete $\beta$-cell degeneration, and type II diabetes is characterized by insulin resistance and $\beta$-cell dysfunction. This eventually leads to chronic hyperglycemia, and a constant exposure to high blood glucose levels leads to significant complications. Of the extensive list of complications, the most significant resulting from hyperglycemia includes hypertension, ketoacidosis, nephropathy, neuropathy, and most notably heart disease.

\subsubsection{Hypertension}

Hypertension is a very common condition in diabetics, and especially common in patients with type II diabetes. Normal blood pressure is commonly defined as $120 / 80 \mathrm{mmHg}$ for people without diabetes, and 130/80 $\mathrm{mmHg}$ for people with diabetes; however, stage one hypertension begins with a consistent blood pressure reading of 140-160/90-100 mmHg. Hypertension increases the risk of macrovascular and microvascualr complications, specifically cardiovascular 
diseases including stroke, coronary artery disease, peripheral vascular disease, retinopathy, nephropathy, and even neuropathy [35]. In fact, when hypertension coexists with diabetes, the risk of stroke or cardiovascular disease is doubled, and the risk of developing renal disease increases five to six times compared to hypertensive patients without diabetes [36]. In type I diabetics, hypertension is usually a mark of diabetic nephropathy, and these two disorders aggravate each other. In type II diabetics, hypertension is associated with complications such as microalbuminuria, obesity, insulin resistance, dyslipidemia, hypercoagulation, and hyperuricemia [36]. Basic treatment of hypertension involves dietary and lifestyle modifications such as weight loss, exercise, reduced sodium intake, and an improved balanced diet. Pharmacologic therapy is another method with the use of drugs, such as ACE-inhibitors, diuretics, and $\beta$-blockers [37].

\subsubsection{Ketoacidosis}

Diabetic ketoacidosis is the most common hyperglycemic complication in patients with diabetes mellitus [38]. It occurs when there are dangerously high levels of ketone acids in the blood. The combination of uncontrolled hyperglycemia, metabolic acidosis, and increased body ketone concentration characterize diabetic ketoacidosis. This is a serious condition more commonly found in type I diabetics than type II, and can lead to a diabetic coma or even death if not treated properly. Diabetic ketoacidosis occurs when glucose is not available as a fuel source for the body; thus, fat is used instead. The byproducts of fat breakdown are ketones and acid, dropping the blood $\mathrm{pH}$ and making it more acidic than usual. The basic mechanism behind ketoacidosis is mostly insulin deficiency along with elevated levels of counter-regulatory hormones, such as glucagon, cortisol, and growth hormone [39]. The treatment of diabetic 
ketoacidosis involves correcting the fluid and electrolyte imbalances with saline, water, and potassium, normalizing the blood glucose levels with insulin, and correcting the acid-base disturbances using insulin or bicarbonate [40].

\subsubsection{Nephropathy}

Renal dysfunction in diabetes, known as diabetic nephropathy, is a progressive disease that occurs as a result of both direct and indirect effects of hyperglycemia and hypertension [41]. It is a multifactorial disorder that results from the interaction of both environmental and genetic factors [42]. The risk of developing diabetic nephropathy is only partially determined by glycemic control and is highly influenced by genetic susceptibility [43]. It is a progressive renal disease caused by angiopathy of capillaries in the kidney glomeruli, resulting in the leakage of albumin protein into the urine. It remains the leading cause of end-stage renal disease and develops in 20-25\% of type I diabetic patients [44]. Although the risk of diabetic nephropathy in type I diabetes is similar to that of type II diabetes, it is most commonly associated with type II diabetics [45] due to the low level of glycemic control and the high level of hypertension associated with type II diabetes. Diabetic nephropathy occurs first with glomerular hyperflitration (due to the thickening of the glomerular basement membrane) followed by increased urinary albumin excretion known as microalbuminuria - the leakage of albumin proteins into the urine [46]. Once HA1C values pass the threshold value of $8.0 \%$, there is a significant increase in the chance of microalbuminuria [47]. Treatment of diabetic nephropathy involves control of blood glucose levels to prevent chronic hyperglycemia, plus the management of hypertension that is known to aggravate the disorder even further. Pharmacological therapy, 
with the use of ACE-inhibitors, is another option to reduce the hypertension and also reduce microalbuminuria to control proteinuria in both type I and type II diabetics.

\subsubsection{Neuropathy}

A common complication of diabetes, diabetic neuropathy occurs due to damaged nerves as a result of hyperglycemia. Diabetic neuropathy affects both type I and type II diabetics, but progresses more rapidly and has more severe consequences in type I diabetics [48]. There are two common types of nerve damage; the first is peripheral diabetic neuropathy (PDN) that has devastating complications to the cranial nerves and spinal nerves causing tingling, pain, numbness, or weakness to the hands and feet and is the leading cause of foot amputations. PDN occurs due to complex interactions among multiple hyperglycemia-initiated mechanisms, impaired insulin signaling, inflammation, and disturbances of fatty acid and lipid metabolism [49]. The second common type of nerve damage is called autonomic neuropathy that significantly influences the nerves that regulate heart, gastrointestinal, and genitourinary system. It also affects the regulatory function of microcirculation that may lead to different late diabetic complications such as gastroparesis, bladder dysfunction, erectile dysfunction, and retinopathy [48]. Parasympathetic nerve fibers are affected first, leading to a relative increase in sympathetic tone resulting in the increase in resting tachycardia. This decrease in parasympathetic tone may also be responsible for worsening ischemia in diabetics due to the exaggerated coronary vasoconstriction [50]. Despite numerous drug trials and studies, other than strict glycemic control, there are no other methods of treatment to slow the progression of diabetic neuropathy [51]. 


\subsubsection{Cardiovascular Disease}

Diabetes is the seventh leading cause of death in the United States, and much of that is a result of cardiovascular disease. Type I and type II diabetes are independent risk factors for atherosclerotic diseases, stroke, and peripheral arterial disease (PAD) [52]. These macrovascular complications are coupled with microvascular complications such as retinopathy, neuropathy, and nephropathy making diabetes mellitus, according to the American Heart Association, a cardiovascular disease on its own. Chronic high blood pressure (hypertension) and abnormalities in glucose metabolism are the mechanisms by which diabetes contributes to adverse heart dysfunction and failure [53]. There is evidence that adequate glycemic control may improve cardiac metabolism and myocardial function in diabetics with a high risk of heart disease; therefore, there is a direct relationship between hyperglycemia and cardiovascular disease in type I and type II diabetic patients [54].

\subsubsection{Coronary Artery Disease}

Out of all cardiovascular diseases, diabetes mellitus is a major contributor to the development and the manifestations of coronary artery disease (CAD). Patients with CAD and diabetes mellitus represent a particularly high-risk population because of the specific characteristics associated with the atherosclerotic disease [55]. CAD occurs late in the third or fourth decade of a diabetic's life, regardless of whether diabetes developed early in childhood or during late adolescence. While men are generally more susceptible to CAD than women, studies have shown that women with diabetes are at especially high risk for CAD, relatively more so than men with diabetes [56]. The disease results in the narrowing of coronary arteries due to atherosclerosis - the build-up of atheromatous plaques (Figure 7). 


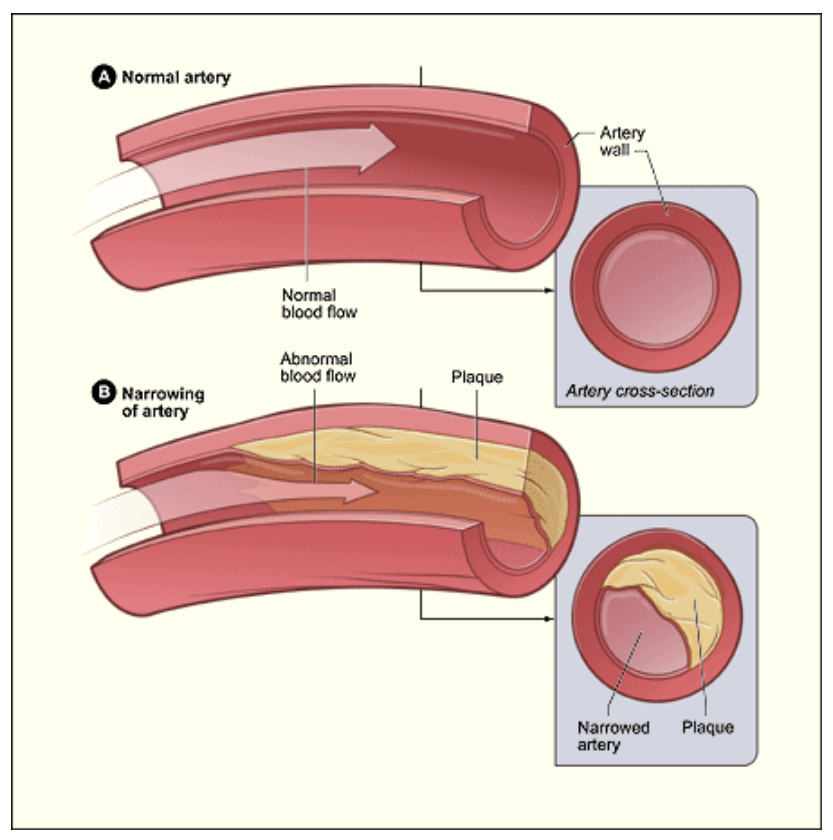

Figure 7 - Coronary artery disease results when a normal healthy coronary vessel, (a) narrows due to atherosclerosis and plaque build-up, and (b) leads to inadequate blood flow through the vessel (Permission granted from the National Heart, Lung, and Blood Institue as a part of the National institute f Health and the US Department of Health and Human Services).

Risk factors that emerge as predictors for coronary artery disease in type I diabetics include nephropathy and autonomic neuropathy [57]. Nephropathy has always emerged as a major predictor [58], but coronary artery disease is still a risk even if the patient does not have nephropathy. Diabetic nephropathy is associated with an atherogenic lipoprotein profile that includes elevated levels of low-density lipoprotein (LDL) and very-low-density lipoprotein (VLDL), and decreased levels of high-density lipoprotein (HDL) [59]. Several mechanisms contribute to the atherosclerotic process, including hypertension, lipid abnormalities, fibrinolysis, and coagulation alterations [60]. As discussed before, hypertension is frequently present in patients with nephropathy and can intensify coronary artery disease in patients with type 1 diabetes. As for patients with type II diabetes, hypertension, hyperglycemia and increased concentrations of LDL along with decreased concentrations of HDL are independent risk factors that contribute to the development of coronary artery disease [61]. 
Medical therapies for treating coronary artery disease in patients with diabetes include insulin therapy, aspirins, $\beta$-blockers and ACE inhibitors. Lifestyle modifications should be taken as well by reducing cholesterol levels, treating chronic hypertension, and controlling glycemic concentrations. With the increase of endothelial dysfunction in the coronary blood vessels due to hyperglycemic conditions, diabetics are more susceptible to CAD, which commonly manifests itself into unstable angina or worse - myocardial infarction. CAD is a significant complication related to diabetes and is the leading cause of death in the world. Due to the significant and dominance of the heart disease, this thesis will focus on the involvement of CAD with diabetes mellitus.

\subsection{Treatments for Diabetics with Coronary Artery Disease}

Regardless of whether a patient has diabetes or not, revascularization is a common treatment for patients with coronary artery disease. Treatment procedures include coronary angioplasty, coronary bypass surgery, and coronary stent placement. Numerous studies show that patients with diabetes experience a greater need for repeat revascularization (angioplasty, bypass, or stent replacement), a higher rate of cardiac events, and lower overall survival following the coronary therapy procedures compared to their non-diabetic counterparts [62]. Diabetic patients commonly have more pretentious coronary artery involvement than non-diabetics, which probably contributes to their less favorable outcome following revascularization [62]. Each of these treatments will be summarized below, along with a description of the additional impact of diabetes on the treatment's success. 


\subsubsection{Angioplasty}

Coronary angioplasty (Figure 8) is a medical procedure where a balloon catheter is deployed to open a coronary artery that has been narrowed by plaque build-up from atherosclerosis. Angioplasty provides effective relief of angina - chest pain that occurs when a part of the heart is not receiving enough oxygenated blood - and is usually successful in patients with or without diabetes mellitus. However, the great long-term effects of angioplasty are greatly limited by the increased rates of restenosis in patients with diabetes (Figure 9) [63]. New angioplasty devices have been used (laser angioplasty and directional coronary atherectomy), but have failed to show any major improvement on the high rate of restenosis among patients with diabetes. 


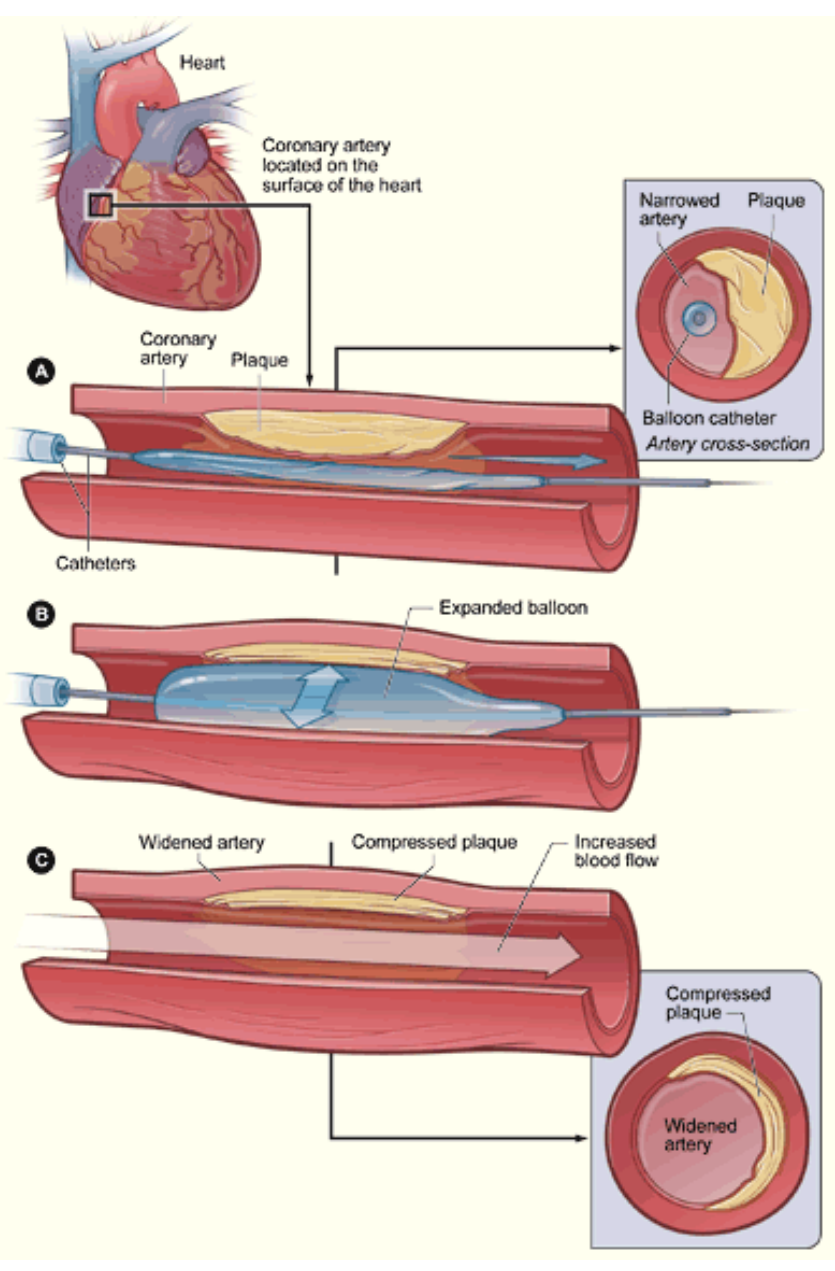

Figure 8 - With the build-up of plaque in a coronary artery, coronary angioplasty is a medical procedure where, (a) a balloon catheter is inserted to the site of plaque buildup, (b) the balloon is inflated and pushes the plaque against the blood vessel wall, and (c) proper blood flow is restored to the artery (Permission granted from the National Heart, Lung, and Blood Institue as a part of the National institute f Health and the US Department of Health and Human Services). 


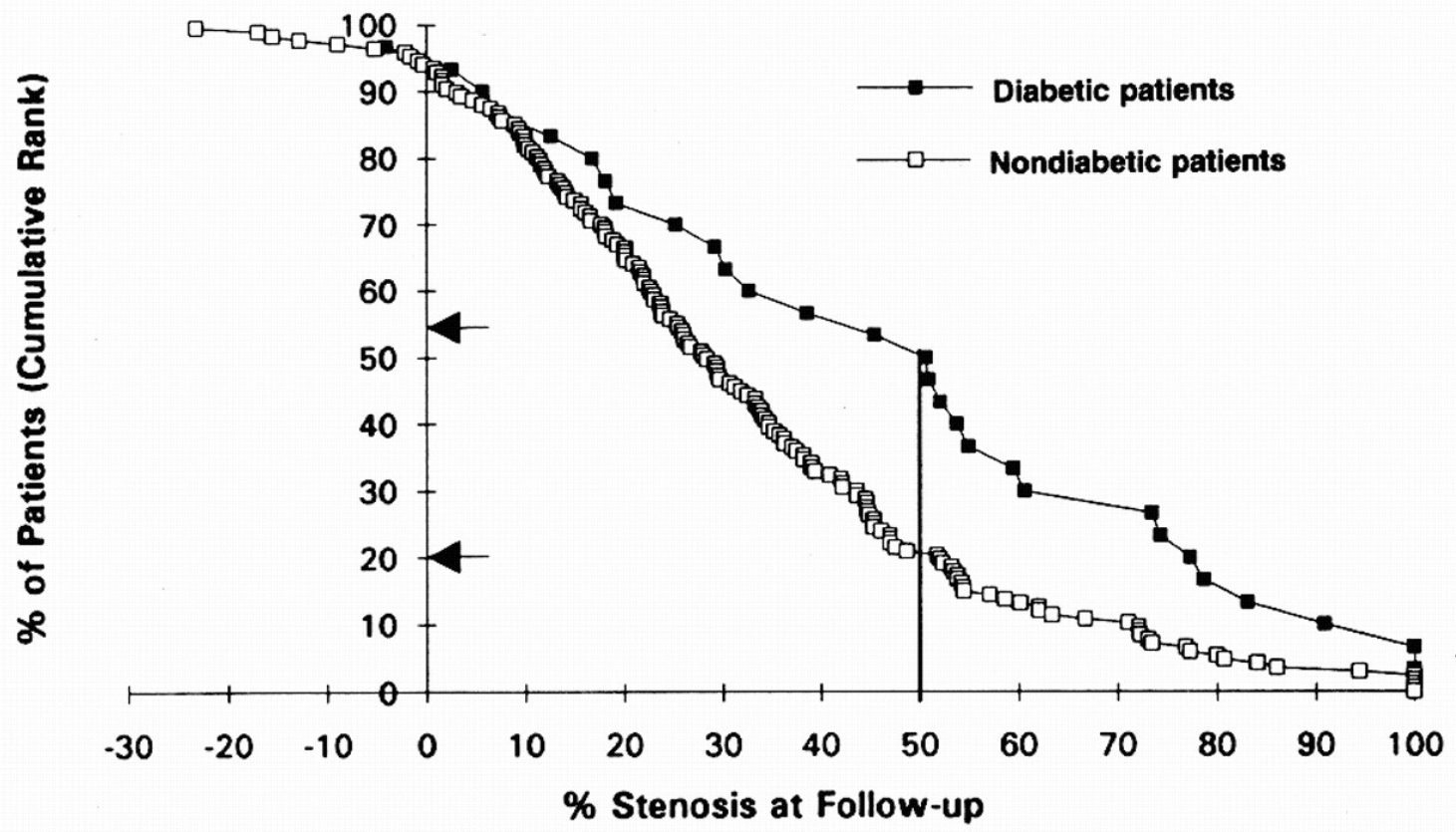

Figure 9 - The graph above displays that angiographic restenosis (50\% diameter stenosis) was significantly greater in diabetic patients than non-diabetic patients (Permission to reprint by [63]).

Diabetics may exhibit an accelerated form of intimal hyperplasia and atherosclerosis in response to the vascular injury caused by balloon deployment. A study using intravascular ultrasound found exaggerated intimal proliferation in patients with diabetes at the site of arterial injury caused by angioplasty, and this response was striking in restenosis lesions [64].

Hyperinsulinemia, common in type II diabetics with insulin resistance, may contribute to the atherogenic process since insulin may directly promote smooth muscle cell proliferation, and the procoagulant state in diabetics may contribute directly to the progression of restenosis [65]. In summary, while there are some similarities in the angiographic outcomes after successful angioplasty in diabetics and non-diabetics, the vascular injury caused by treatment of balloon angioplasty contributes to the increased risk of restenosis in the treated blood vessel. 


\subsubsection{Coronary Artery Bypass Surgery}

Coronary artery bypass surgery involves rerouting blood around a clogged artery to reinstate proper blood flow to the heart. This is an extremely invasive procedure where surgeons take a segment of a healthy blood vessel (preferably a portion of the great saphenous vein) and make a new path around the blocked region on the coronary artery for blood to flow freely to the heart muscle. Coronary artery bypass surgery is effective in relieving angina symptoms in patients with and without diabetes, but with respect to vein grafts, several reports have noted decreased survival of vein grafts in patients with diabetes [66]. Intimal proliferation, that causes luminal loss in the first years following bypass surgery with venous vessels, may be accelerated in patients with diabetes mellitus [67]. Studies have shown that restenosis after coronary artery bypass grafting is more common among diabetics versus non-diabetics at 1-year angiography mainly because of more graft failures in diabetics [68]. It is believed that the decreased success of coronary artery bypass grafts in diabetics and the increased short-term mortality in diabetics is due to metabolic abnormalities, such as hyperglycemia, and cardiac complications (especially in type II diabetics), which include hypertension and a possible history of peripheral vascular disease or stroke [74]. Thus, with reduced availability of healthy vein graft replacements within diabetic patients, coronary artery bypass surgery is becoming a less common method of treatment for CAD patients with diabetes mellitus.

\subsubsection{Coronary Stent Placement}

Coronary stent placement involves the placement of a man-made wire mesh tube during angioplasty that expands and props open the coronary artery that is narrowed or blocked by atherosclerotic plaque build-up. During the percutaneous coronary intervention, the same steps 
as other angioplasty procedures are performed as the stent expands and locks into place when the balloon inflates. Although bare metal stents have improved outcomes among patients undergoing percutaneous coronary interventions, their efficacy has been limited by the development of in-stent restenosis [69] (Figure 10). Studies have shown that the rate of restenosis increases in patients with both type I and especially type II diabetes, and specific angiographic characteristics, such as vessel size and stent type (bare metal vs. drug-eluting), are predictive of a higher rate of restenosis and a need for repeated revascularization procedures after the initial stent implantation [70]. Although vessel recoil may also contribute to decrease of lumen size, the increased restenosis rate in patients with diabetes, following stent placement, is due primarily to the enhanced proliferation of smooth muscle cells, because coronary artery "stenting" decreases the elastic recoil and vascular spasm at the treated site [63].

Restenosis is the healing response of the arterial wall to mechanical injury (stent implantation in this case) and is composed of two main processes - neointimal hyperplasia (i.e., smooth muscle migration/proliferation, extra cellular deposition) and vessel remodeling [71]. From a few animal study observations, neointimal hyperplasia is a general wound-healing response - platelet aggregation, inflammatory cell infiltration, release of growth factors, medial smooth muscle cell modulation and proliferation, proteoglycan deposition, and extracellular matrix remodeling as major milestones in the temporal sequence of the response [72]. Further research added that thrombosis often occurred as a cause of smooth muscle cell proliferation [73]. The adhesion of circulating leukocytes to endothelial cells plays an important role in the initiation of CAD and certainly in the occurrences of restenosis in stent-implanted diabetic CAD patients [74]. Levels of cellular adhesion molecules, particularly intracellular adhesion molecule (ICAM)-1 and vascular cellular adhesion molecule (VCAM)-1, have been found at increased 
levels in diabetic patients [75, 76]. Notably, hyperglycemia was shown to condition the concentration of these cellular adhesion molecules acutely [77, 78]. In support of this, recent studies have shown that levels of VCAM-1 and ICAM-1 increase in high glucose environments; thus, the hyperglycemic environment in diabetics is a significant contributing factor to the high rate of in-stent restenosis $[77,79]$.

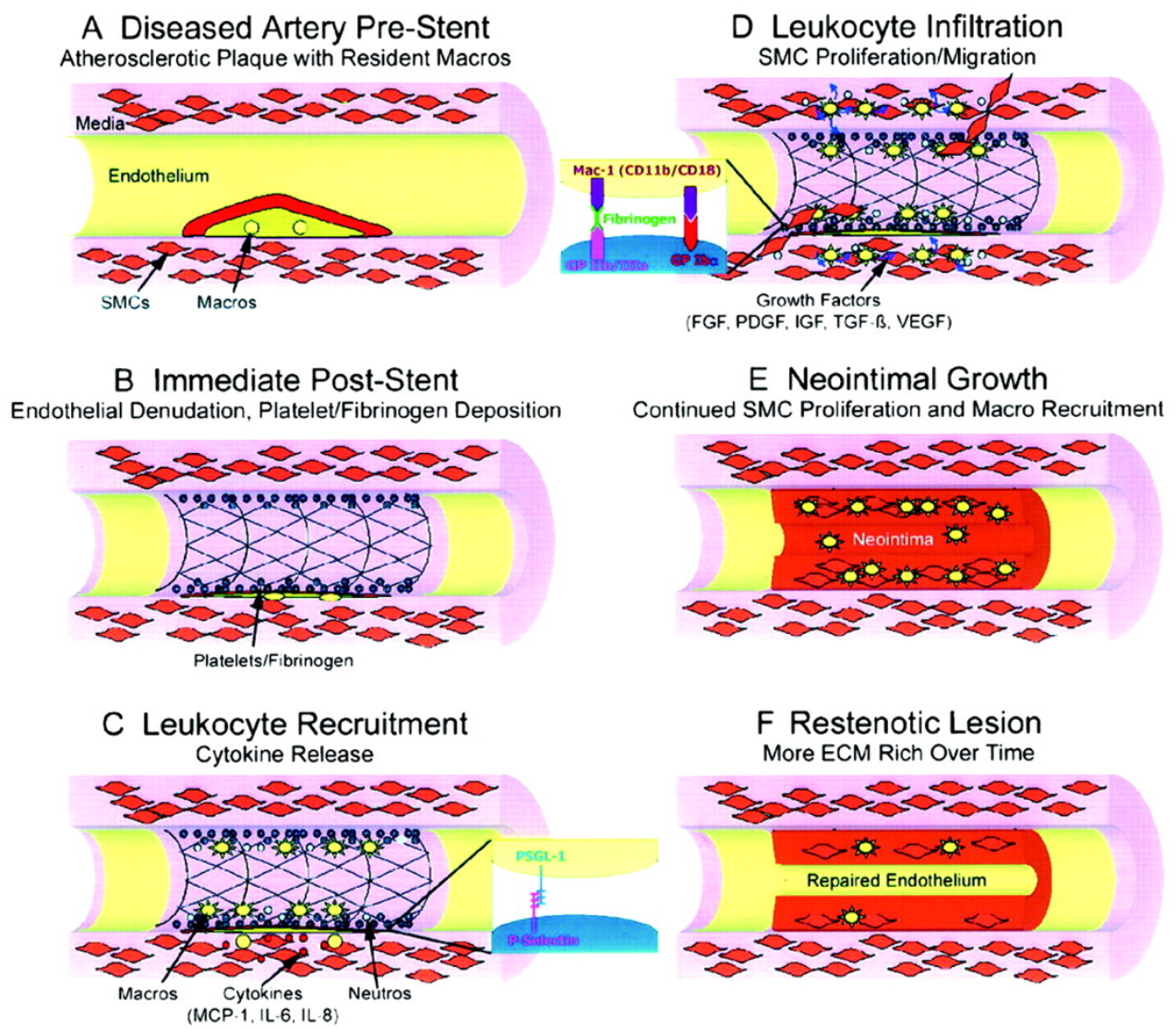

Figure 10 - The diagram shows the progression of in-stent restenosis as a vessel injury response resulting in the proliferation of smooth muscle cells, the expression of vessel adhesion molecules, and the adhesion of leukocytes (Permission to reprint from [71]).

The development and use of drug-eluting stents reduced the risk of restenosis, target lesion revascularization, and myocardial infarction as compared to bare-metal stents; however, the risk of very late thrombosis is higher with drug-eluting stents than with bare metal stents [80]. There are two primary types of drug-eluting stents used in the market - sirolimus-eluting 
stents and paclitaxel-eluting stents. Recent studies have compared the use of sirolimus-eluting stents and paclitaxel-eluting stents in diabetics and suggest that the sirolimus-eluting stent is associated with a decrease in late lumen loss and a reduced risk of restenosis [81]. Implantation of drug-eluting stents has emerged as the predominant revascularization strategy in diabetic patients, especially with the improved reduction of in-stent restenosis [82].

\subsection{Coronary Stent Treatment in the Diabetic Population}

\subsubsection{Restenosis in In Vivo Diabetic Studies}

The increased atherothrombotic risk in patients with diabetes is related to their proinflammatory and pro-thrombotic status [162]. Coronary artery plaques of diabetic patients with acute myocardial infarction had significantly higher necrotic core percentages and lower fibrofatty tissue percentages than non-diabetics $[163,164]$. In addition, diabetic patients with stable angina and significantly larger dense calcium in culprit lesions [164, 165]. The pathogenic mechanism of restenosis after drug-eluting stent implantation under hyperglycemia is still not completely understood, and most reports have been conducted in a clinical setting, with little research done on animals. However, a recent rat study conducted showed increased thrombus, inflammatory cell infiltration, and neointimal hyperplasia (NIH) without change of smooth muscle cell number after drug-eluting stent implantation in diabetic rats $(493.3 \pm 85.8 \mathrm{mg} / \mathrm{dL}) \mathrm{vs}$. non-diabetic rats $(140.0 \pm 8.5 \mathrm{mg} / \mathrm{dL})$ (Figure 11) [162]. This discovery supported that the main mechanism of restenosis after drug-eluting stent implantation under hyperglycemic conditions was initial thrombus and inflammatory cell accumulation - causing a delay of 
reendothelialization - with changes of extracellular matrix rather than smooth muscle cell proliferation [162].
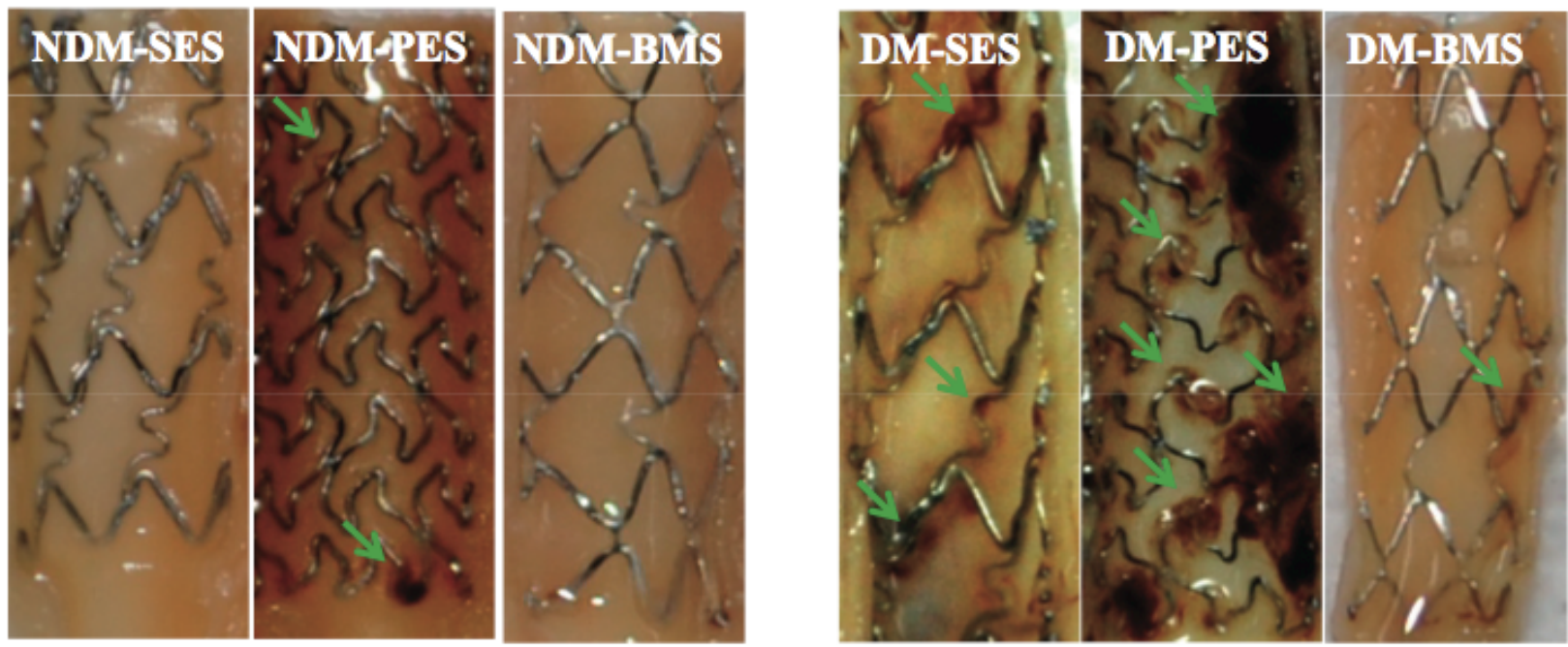

Figure 11 - Increased thrombus was located in the surrounding region of the stent strut (green arrows) of rats with diabetes vs. rats without diabetes (Permission to reprint from [162]).

In another recent diabetic rat model study showed the development of in-stent restenosis enhanced by long-term type I diabetes in BBDP rats [166]. Quantitative analysis supported that long-term diabetes resulted in a $32 \%$ increase in surface neointima compared to non-diabetic rats BBDP rats (Figure 12) [166]. Though the underlying mechanism(s) were not studied, this study, however, suggested that long-term diabetes increases the proliferative and migratory capacity of medial and neointimal smooth muscle cells [166].

Future studies aimed at identification of cellular and molecular mechanisms involved with increased restenosis in diabetic animal models are warranted to improve therapeutic interventions. 


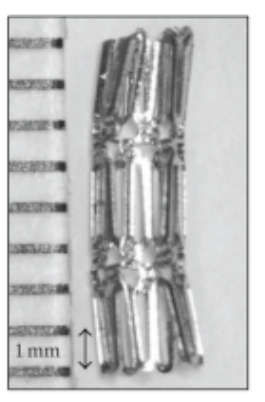

(a)
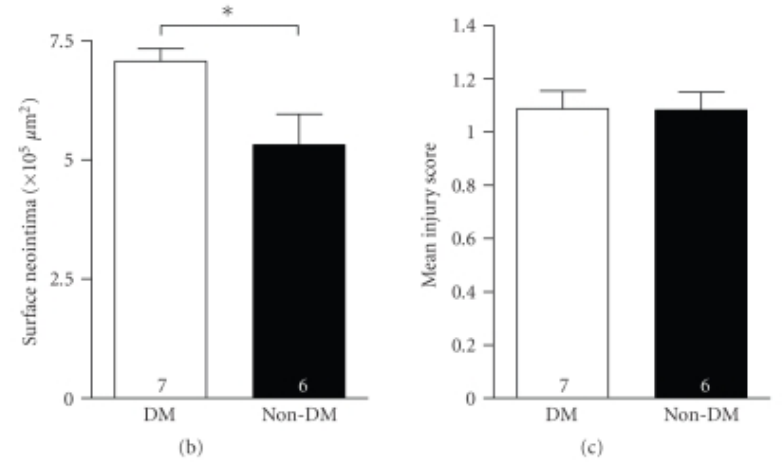

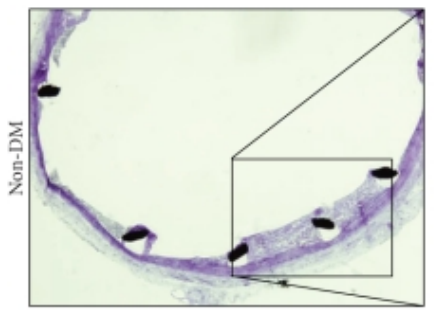

(d)

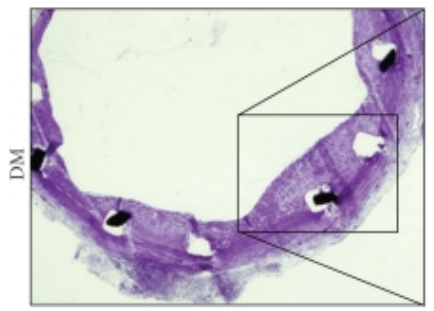

(f)

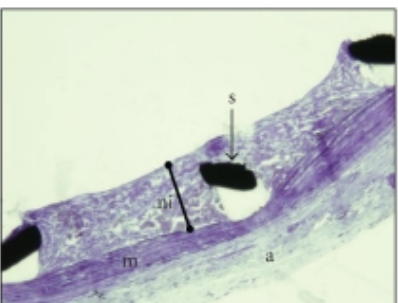

(e)

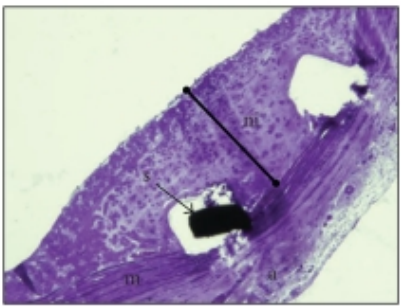

(g)

Figure 12 - Long-term type I diabetes in BBDP rats is associated with enhanced in-stent restenosis four weeks after stenting the abdominal aorta (Permission to reprint by [166]).

\subsubsection{Clinical Results of Diabetic Coronary Stent Implantation}

\subsubsection{Type I Diabetics vs. Type 2 Diabetics}

In-stent restenosis is common occurrence in CAD patients, and is even greater in CAD patients with diabetes. However, is there a difference in the rate of restenosis between type I and type II diabetics? After reviewing the literature, no significant data has been reported showing any difference between the rates of in-stent restenosis between type I and type II diabetics. Studies do report that a chronic hyperglycemic state in the coronary vessels of diabetics 
contributes to greater progression of $\mathrm{CAD}$ and an increased rate of restenosis, and since type I diabetics have much more control over their levels of hyperglycemia compared to type II diabetics, it would make sense that the occurrence of in-stent restenosis is greater in type II diabetics than in type I diabetics. This also supports the fact that type II diabetics are much more susceptible to cardiovascular complications - thus, needing more revascularization treatments which would produce higher cases of restenosis in that population compared to the other.

\subsubsection{Bare Metal Stents vs. Drug-Eluting Stents in the Diabetic Population}

The next studied comparison involved researching whether or not any significant difference existed in the rate of restenosis between diabetics with implanted bare metal stents and diabetics with implanted drug-eluting stents. Recent studies compared and reported the reduced rate of in-stent restenosis for both types of drug-eluting stents (sirolimus and paclitaxel) compared to bare metal stents. The results of a follow-up study concurred with the previous results in that drug-eluting stents lowered the restenosis rate by $50 \%$ in diabetic patients with one implanted drug-eluting stent, compared to diabetic patients with one implanted bare metal stent [83]. Although, on average, sirolimus-eluting stents and paclitaxel-eluting stents are associated with a significant reduction in target lesion revascularization compared to bare metal stents, the overall mortality and cardiac mortality of all three stents are quite similar [84]. However, as mentioned before in 1.3.3, when comparing paclitaxel-eluting against sirolimus-eluting stents in patients with diabetes mellitus, the use of sirolimus-eluting stents is associated with a decrease in late luminal loss and a reduced rate of restenosis compared to the use of paclitaxel-eluting stent $[85,86]$. 


\subsubsection{Summary of Clinical Results}

To summarize, diabetic patients (mostly type II diabetics) with coronary artery disease are highly susceptible to in-stent restenosis after stent implantation, due to the vascular aggravation induced by the chronic hyperglycemic environment. There is an effort to improve stent revascularization therapy for the diabetic population, but there is limited information from clinical trials to guide manufacturers in the right direction for better treatment. Thus, the following section will reveal the purpose and goal of this thesis, and also touch upon the overall perspective on how this study supports the development of improved in vitro testing of coronary stent treatments.

\subsection{Summary and Aims of the Thesis}

Diabetes mellitus is an amazing disease to study - from its severe, deadly nature before the dramatic discovery of insulin, to its variability as distinct types, it is a disease that seems to produce new obstacles in treatment. However, its treatment coincides with the treatments of so many other severe complications. The chronic hyperglycemic state exhibited by type II diabetics is the root cause of the numerous complications associated with the disease - the most severe complication being coronary artery disease; the leading cause of death in the world. The vessel damage caused by hyperglycemia can lead to atherosclerotic plaque build-up in the coronary vessels of the heart, and stent implantation is the most popular treatment to eliminate the blockage. However, patients with diabetes experience a high rate of in-stent restenosis - the reformation of the atherosclerotic blockage - due to the vascular injury caused by the deployment of the stent. 
Stent designs are constantly modified and improved to meet the demands of cardiovascular treatment, and it is important for stent producers to be able to evaluate the practicality and mechanics of the designs in a time and cost efficient manner. While in vivo animal models and clinical testing are vital for product evaluation, these are extremely expensive and time consuming; thus, it would be beneficial to be able to test and evaluate stents beforehand in a controlled in vitro setting that accurately replicates the environment of a coronary blood vessel. A blood vessel model that accurately replicates the anatomy and physiology of a native blood vessel - a blood vessel mimic (BVM) - could meet this need

The primary purpose of BVM research has been to evaluate the endothelialization of bare metal and coated stents [87]. In the case of diabetes mellitus, an in vitro model of a diabetic coronary blood vessel would be extremely useful to evaluate the failure behind the implantation of bare metal and drug-eluting stents in the diabetic population with coronary artery disease, and aid the improvement of the vascular treatment for the ailing population.

The goals of this thesis focused on the feasibility of creating and monitoring a hyperglycemic environment that would help develop a diabetic/hyperglycemic BVM. Hyperglycemia is the most significant root cause of the major complications in diabetics; thus, it is no surprise that it was determined to be the basis of a diabetic BVM. However, before diving into the creation of a diabetic BVM, it was necessary to test whether or not an induced hyperglycemic environment could be created and monitored in vitro, and what the effects on cells in culture would be. 
Therefore, this thesis was driven by the hypothesis that a high concentration of glucose can be created and monitored in vitro to replicate the chronic state of hyperglycemia in a diabetic environment. In order to test this hypothesis, the thesis was broken down into three major aims:

1. Establish a protocol to create high glucose solutions in standard cell culture media

2. Establish a protocol to accurately monitor the concentrations of the high glucose solutions

3. Test this monitoring protocol in cell culture over time and determine how the high glucose solutions affect endothelial cells

These aims served the purpose of determining whether or not a feasible hyperglycemic environment can be induced into an in vitro BVM; thus, ultimately allowing the creation of a diabetic blood vessel environment. The potential success of this study would progress towards an improved evaluation of bare metal and drug-eluting stents that currently experience a high rate of in-stent restenosis in diabetic patients with coronary artery disease. 


\section{Chapter 2: Establishment \& Evaluation of High}

\section{Glucose Solutions in Order to Create Hyperglycemic}

\section{Environments}

\subsection{Introduction}

As discussed in Chapter 1, diabetes mellitus is one of the most common and deadliest diseases among Americans. According to the American Diabetes Association, about 23.6 million adults and children in the United States are diagnosed with diabetes - which is $7.8 \%$ of the population [88]. Diabetes is most commonly seen in two forms, type I and type II. Type I diabetes, also known as insulin deficient (or dependent) diabetes, is an autoimmune disease that degrades pancreatic $\beta$-cells; thus, preventing the secretion of insulin. Patients suffering from this type of diabetes are usually treated with subcutaneous insulin injections. Type II diabetes, also known as insulin resistant (or independent) diabetes, occurs due to genetics, poor diet, and poor health. The result is complete dysfunction of pancreatic $\beta$-cells; thus, the body no longer responds to the physiological effects of insulin and results in a chronic hyperglycemic state. Patients suffering from type II diabetes may be treated with insulin along with oral medication; however, significant lifestyle changes involving improved diet and increased exercise are necessary. Out of the $7.8 \%$ of Americans diagnosed with diabetes, about $95 \%$ have type II diabetes making it the more significant and deadly form of the disease [88].

The high death rate associated with diabetes mellitus is due primarily to the complications caused by hyperglycemia - high blood glucose concentration - in the body. The 
body's inability to adequately control its blood glucose levels induces this hyperglycemic environment and can lead to the severe complications that were discussed in section 1.2.5. Due to its severity and significance, replicating a hyperglycemic environment is the desired approach to accurately replicating a diabetic environment for a blood vessel mimic (BVM). The focus of this chapter will revolve around studying the glucose concentrations of a hyperglycemic environment, and the most practical method of replicating this environment for incorporation with a BVM.

\subsubsection{Significance of Hyperglycemia \& Common Complications in Diabetic Patients}

As described in detail in Chapter 1, hyperglycemia is a condition seen in type I and type II diabetics. If not treated properly, the condition can result in a range of complications. The condition usually occurs in type I diabetics if an insufficient amount of insulin has been delivered, but is more prevalent in type II diabetics since the metabolic effect of insulin is ineffective. Miscalculations in carbohydrate counting when adjusting insulin dosages, or daily stresses from life can worsen the hyperglycemic condition. For patients, it is important to constantly monitor their own glucose concentrations and avoid drastic hyperglycemic episodes. It is also important for diabetic patients (especially type I diabetics) to avoid hypoglycemic episodes - a state of extremely low blood sugar - as it can lead to a diabetic coma and even death. Since this study focuses on the effects of hyperglycemia in CAD patients with diabetes, the state of hypoglycemia will not be discussed.

It is extremely important for diabetic patients to treat a severe case of hyperglycemia as soon as it is detected. Failing to do so can first lead to a complication called ketoacidosis, which 
can also lead to a diabetic coma. The human body usually relies on glucose as its natural fuel. Without insulin working effectively, the body is unable to breakdown glucose properly and is required to break down fats as an energy source instead. Once the body breaks down fats, waste products called ketones are produced that the body cannot tolerate at high levels. This results in the body's attempt to rid of the ketones through urine but is usually unable to; thus, leaving an excess that eventually ends up in the bloodstream causing ketoacidosis [89]. This condition is life threatening and requires immediate attention (usual symptoms are shortness of breath, nausea, vomiting, dry mouth, and even a fruity smelling breath) [89].

While lower states of hyperglycemia do not cause severe complications rapidly, a prolonged hyperglycemic condition usually leads to more long-term complications including hypertension, neuropathy, nephropathy, eye complications (retinopathy, cataracts, glaucoma), and most significantly heart disease - notably, coronary artery disease (CAD) [88]. Table 2 lists the most significant complications of hyperglycemia and provides brief descriptions. 
Table 2 - Common complications associated with diabetes mellitus resulting from hyperglycemia

\begin{tabular}{|c|c|}
\hline Complication & Description \\
\hline Ketoacidosis & $\begin{array}{l}\text { Diabetic coma; increased blood ketone } \\
\text { concentration \& metabolic acidosis }\end{array}$ \\
\hline Hypertension & Increased blood pressure \\
\hline Neuropathy & Impaired nervous system signaling \\
\hline Nephropathy & $\begin{array}{l}\text { Kidney disease leading to protein leakage } \\
\text { into urine }\end{array}$ \\
\hline Retinopathy & $\begin{array}{l}\text { Decreased vision or blindness due to } \\
\text { damages to retinal vasculature }\end{array}$ \\
\hline Glaucoma & $\begin{array}{l}\text { Disease affecting optic nerve and loss of } \\
\text { retinal ganglion cells }\end{array}$ \\
\hline Heart Disease/Coronary Artery Disease & $\begin{array}{l}\text { Disease of the coronary artery involving } \\
\text { accumulation of atherosclerotic plaques } \\
\text { within blood vessel walls }\end{array}$ \\
\hline
\end{tabular}

\subsubsection{Vascular Damage Induced by Hyperglycemia}

As noted before, microvascular diseases are extremely prevalent in diabetic patients [91], and these alterations to the vasculature are the most common causes of mortality in diabetics [90]. Hyperglycemia is associated with endothelial cell dysfunction and reduced neovascularization - essential steps for wound healing and prevention of cardiovascular ischemia [92]. Studies have demonstrated that in patients with cardiovascular risk factors, such as the complications associated with diabetes, the number of endothelial progenitor cells is reduced and their functions are impaired [93]. For example, several animal models of diabetes showed 
increased vascular permeability [94], alterations in erythrocyte velocity [95], entrapment of leukocytes in microcirculation [96, 97], and morphological alterations, such as changes in vascular density [95]. All these alterations are mainly described as a result of hyperglycemia $[98,99]$. One study has illustrated that in type I diabetes, endothelial progenitor cell numbers are inversely correlated with HbA1C levels, demonstrating that a high degree of blood glucose concentration directly affects endothelial cell proliferation and differentiation [100].

Further studies with diabetic mice showed a decrease in vascular density; however, there was also an increase in vessel diameter (Figure 13) [90]. The increase in vessel diameter resulted in the combination of an unchanged blood flow rate and a lower vascular density, therefore, leading to decreased tissue perfusion in subcutaneous tissue and an impaired supply of oxygen and nutrients [90]. This explains the impaired wound healing seen in diabetics as mentioned previously. The increase in vessel diameter and the reduction in vessel density correlated with the levels of hyperglycemia represented by the blood glucose levels and body weight of the diabetic mice. Additionally, these correlations support the hypothesis that impaired glycemic control progressively leads to functional and morphologic alteration in the vasculature of early-stage diabetes [90]. 

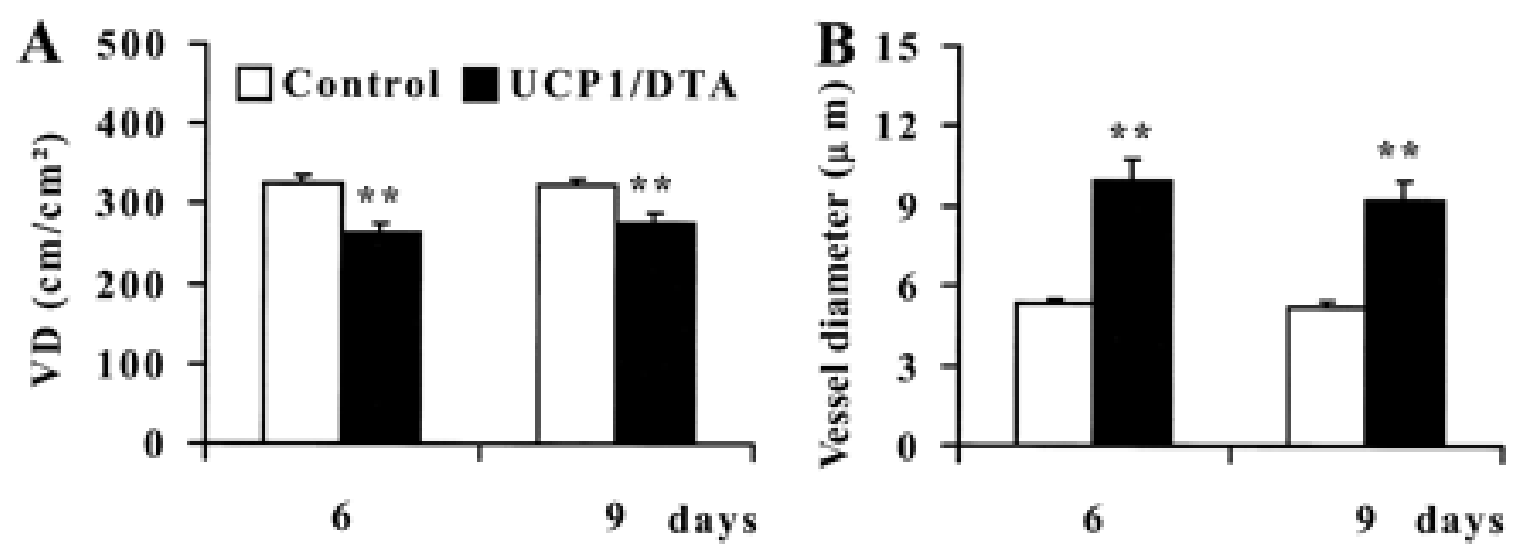

Figure 13 - A study by Algenstaedt et al. showed that diabetic mice experienced a decrease in vascular density, but an increase is vascular diameter with an increase in glycemic concentration (Permission to reprint from [90]).

Despite the drastic impact of hyperglycemia on endothelial dysfunction and microcirculatory properties, more needs to be understood about the alterations of endothelial cells during the development of diabetes. Recall, diabetics with CAD have a high rate of in-stent restenosis after implantation. With all of the supporting information, it is reasonable to propose that the high rate of restenosis in diabetics is the result of the chronic hyperglycemic environment of their blood vessels.

\subsubsection{Molecular Mechanisms of Glucose-Mediated Vascular Damage}

Four main mechanisms have been hypothesized on how hyperglycemia causes diabetic complications. These four mechanisms are: increased polyol pathway flux; increased advanced glycation end-product (AGE) formation; activation of protein kinase $\mathrm{C}$ (PKC); and increased hexosamine pathway flux (Figure 14) [167]. 


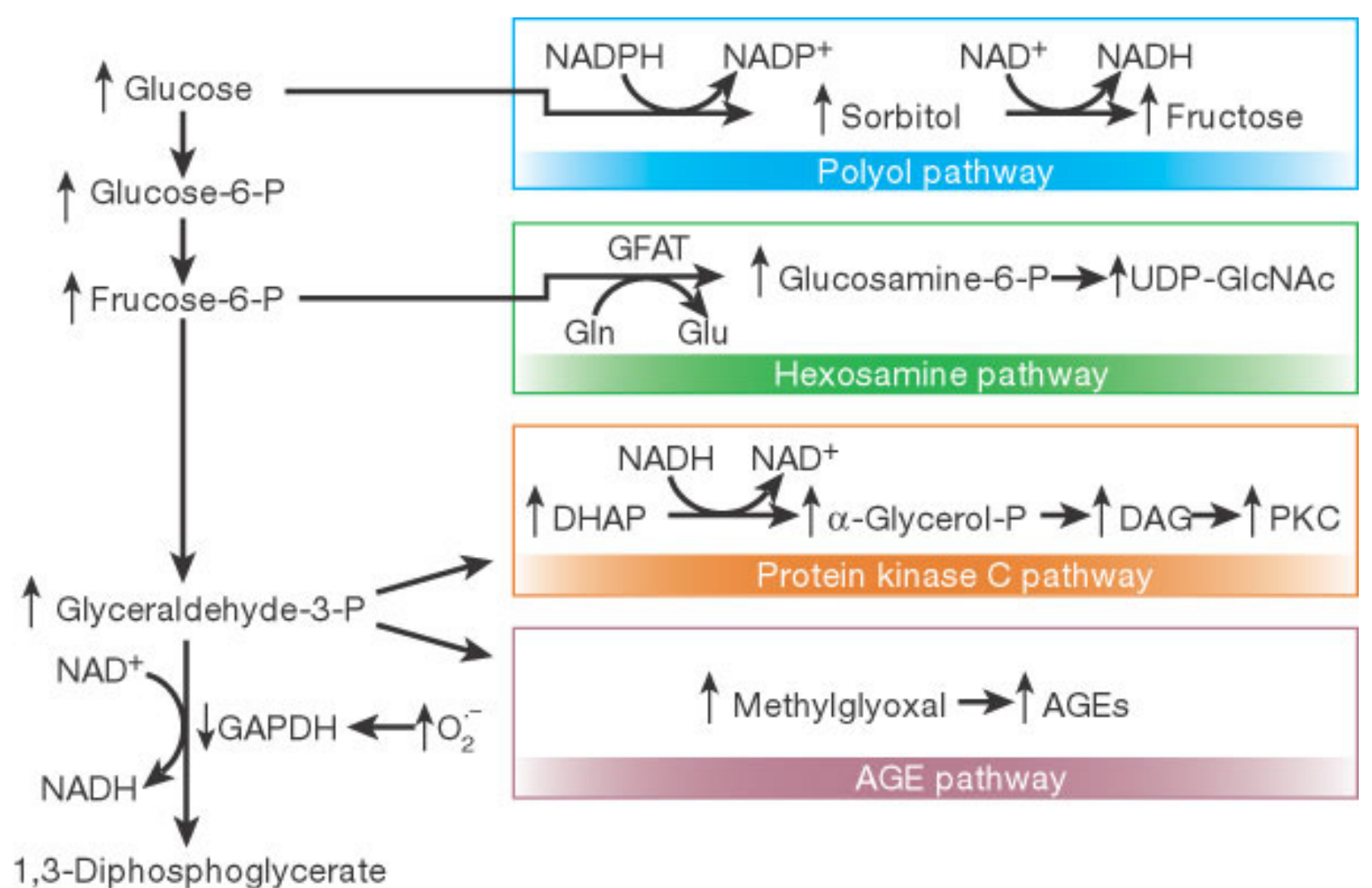

Figure 14 - Potential mechanisms by which hyperglycemia-induced mitochondrial superoxide overproduction activates four pathways of hyperglycemic damage (Permission to reprint by [167]).

For the polyol pathway, aldose reductase is the first enzyme that is responsible for catalyzing glucose. In normal glucose concentrations, a very small percentage of glucose is metabolized via this pathway; however, in a hyperglycemic environment, the increased intracellular glucose concentration results in the conversion of glucose to the polyalcohol sorbitol and a coinciding decrease in NADPH [167]. Mechanisms have been proposed as the root cause of the negative effects induced by a hyperglycemic environment. These mechanisms include osmotic stress induced by elevated sorbitol concentrations; decreased $(\mathrm{Na}++\mathrm{K}+)$ ATPase activity; and an increase in cytosolic NADH/NAD+ and a decrease in cytosolic NADPH [167]. The reduction of glucose to sorbitol by NADPH consumes NADPH, which is required for regenerating reduced glutathione (GSH). Decreased levels of GSH have been found in 
transgenic mice that overexpress aldose reductase; thus, is most likely the mechanisms that results in detrimental consequences via the increased flux through the polyol pathway [168]. However, in vivo studies of inhibition of the polyol pathway have yielded inconsistent results - a five-year study in dogs showed a prevention of diabetic neuropathy but failure to prevent retinopathy or thickening of capillary basement membrane in the retina and kidney, with the inhibition of aldose reductase [169].

Advanced glycation end-products (AGEs) have been found at excessive concentrations in diabetic retinal vessels [170] and renal glomeruli [171]. Additionally, it now seems likely that intracellular hyperglycemia initiates the formation of intracellular and extracellular AGEs [172]. Intracellular auto-oxidation of glucose to glyoxal [173], decomposition of glucose-derived 1amino-1-deoxyfructose lysine adducts to 3-deoxyglucosone, and fragmentation of glyceraldehyde -3-phosphate and dihydroxyacetone phosphate to methylglyoxal [174] can cause an acrease in AGEs. The production of intracellular AGE precursors damage target cells by three general mechanisms. First, intracellular proteins modified by AGEs have altered function; second, extracellular matrix components modified by AGE precursors interact abnormally with other matrix components and receptors for matrix proteins on cells; and third, plasma proteins modified by AGE precursors bind to AGE receptors on endothelial cells, mesangial cells and macrophages, inducing receptor-mediated production of reactive oxygen species [167]. For endothelial cells specifically, fibroblast growth factor is one of the main AGE-modified proteins [175]. Additionally, AGE formation alters the functional properties of several matrix molecules - intermolecular AGE crosslinking induces an expansion of the molecular packing on type I collagen [176], which has been shown to decrease elasticity in large vessels from diabetic rats [177]. Lastly, AGE formation on extracellular matrix also interferes with matrix-cell interaction 
[167]. For example, type IV collagen's cell-binding domains are modified by AGE causing a decrease in endothelial cell adhesion [178].

Increased protein kinase $\mathrm{C}(\mathrm{PKC})$ activity is induced by hyperglycemia since hyperglycemia increased diacylglyceral (DAG) concentration; DAG is responsible for the activation of protein kinase $\mathrm{C}$. Activation of $\mathrm{PKC}$ has numerous detrimental consequences. Early studies have shown that the activation of the $\beta$ PKC isoform mediated blood flow abnormalities by depressing nitric oxide production and/or increasing endothelin- 1 activity [167]. Activation of PKC also causes an increased expression of VEGF, which enhances vascular permeability of smooth muscle cells and also induces angiogenesis [167]. Additionally, PKC activity contributes to increased microvascular matrix protein accumulation by inducing the expression of TGF- $\beta 1$, fibronectin, the fibrinolytic inhibitor PAI-1, and type IV collagen, which contributes to capillary and overall vascular occlusion $[179,180]$.

Lastly, excessive intracellular glucose into the hexosamine pathway may also induce several diabetic complications [181]. In this pathway, when glucose is converted to glucose-6phosphate and then converted to fructose-6-phosphate, instead of entering glycolysis, fructose-6phosphate is used to provide substrates for reactions that require UDP- $N$ - acetylglucosamine, such as proteoglycan synthesis and the formation of $O$-linked glycoproteins [167]. Conversion of fructose-6-phosphate to glucosamine with the rate-limiting enzyme GFAT increases hyperglycemia-induced increased in the transcription of TGF- $\beta 1$ and PAI-1; thus, this pathway is also plays an important role in hyperglycemia-induced and fat-induced insulin resistance [182, 183]. Though it is not entirely known how the mechanisms by which increased activity through the hexosamine pathway initiates hyperglycemic-induced increases in gene transcription, there has been an observation that binding sites for the transcription factor $\mathrm{Sp} 1$ regulate the 
hyperglycemia-induced activation of PAI-1 promoter in vascular smooth muscle cells [184].

This suggested that modification of $\mathrm{Sp} 1$ by $N$-acetylglucosamine (G1cNAc) might explain the link between the activation of the hexosamine pathway and the hyperglycemic-induced changes in the transcription of the gene for PAI-1 [167]. Additionally, recent studies have shown hyperglycemia increasing activity of the hexosamine pathway 2.4 -fold in aortic endothelial cells [185]. In addition to transcription factors, many other nuclear and cytoplasmic proteins are modified by $O$-linked G1cNAc, such as the inhibition of eNOS activity by hyperglycemicinduced $O$-acetylglucosaminylation at the Akt site of the eNOS protein [186]. Thus, activation of the hexosamine pathway by hyperglycemia can result in changes to gene expression and protein function, contributing to the pathogenesis of diabetic complications.

Since hyperglycemia is one of the most significant factors in diabetes, high glucose solutions were proposed as the most feasible method of replicating a diabetic environment in a BVM. However, before exploring the creation and evaluation of the hyperglycemic environments, it is essential to first understand the fundamental physiology of blood glucose in the human body, and the basics behind the metabolic dysfunction that results in hyperglycemia.

\subsubsection{Blood Glucose}

\subsubsection{Properties of Glucose}

Glucose is an extremely important monosaccharide - a simple sugar - used as a source of energy and metabolic intermediate for living cells. The name "Glucose" comes from the Greek word glukus, meaning "sweet." The suffix “-ose” denotes sugar [101]. 
The chemical formula for glucose is $\mathrm{C}_{6} \mathrm{H}_{12} \mathrm{O}_{6}$, and its structure makes it an aldohexose, that is, a hexose with an aldehyde at the end (Figure 15) [102]. A hexose is a monosaccharide with six carbon atoms and usually has the chemical formula $\mathrm{C}_{6} \mathrm{H}_{12} \mathrm{O}_{6}$ [102]. An aldehyde is an organic compound containing a terminal carbonyl group (Figure 16) [103]. This is a functional group that contains a carbon atom bonded to a hydrogen atom and double-bonded to an oxygen atom (chemical formula $\mathrm{O}=\mathrm{CH}$ ). In solution, the glucose molecule can exist in an acyclic (openchain) and a cyclic (ring) form. The cyclic form, also referred to as glucopyranose, is a result of the aldehyde carbon atom double-bonded to the hydroxyl group (oxygen bonded to a hydrogen) on the fifth carbon of the open chain - making a six-member ring (Figure 17) [102]. At a neutral $\mathrm{pH}$ of 7 and in solid form, glucose takes its cyclic form.

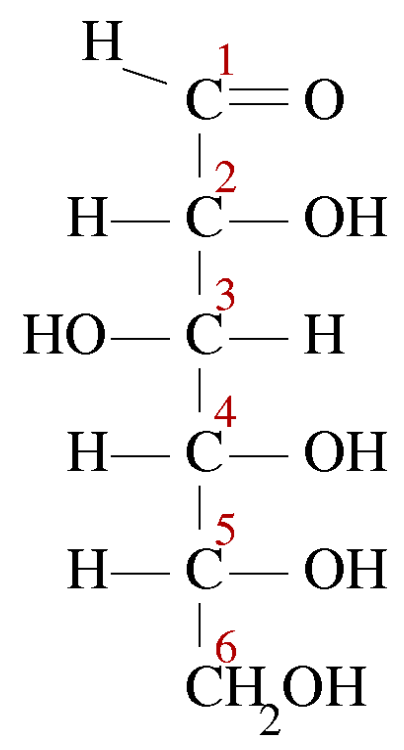

Figure 15 - Glucose is an aldohexose; a hexose with an aldehyde attached at the end (Permission to reprint from [102]). 


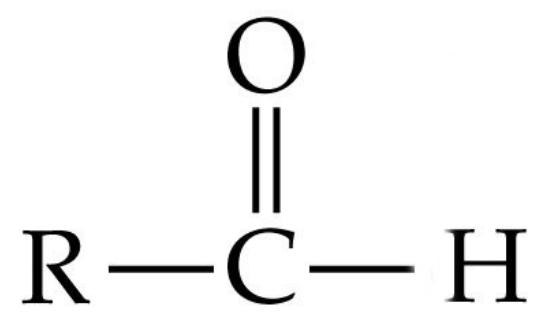

Figure 16 - An aldehyde is an organic compound containing a terminal carbonyl group (Permission to reprint by [103]).
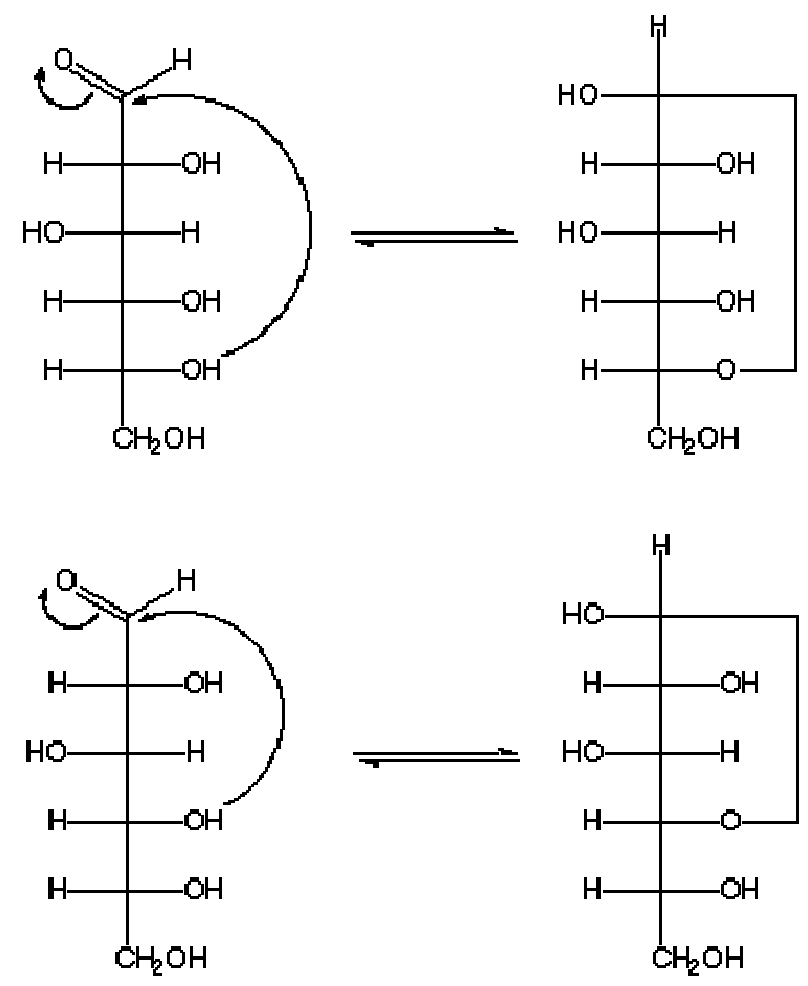

Figure 17 - The cyclic form of glucose, glucopyranose, results from the aldehyde carbon atom double-bonded to the hydroxyl group on the fifth carbon of the open chain (Permission to reprint by [102]).

There are two stereoisomers of the aldohexose sugars referred to as glucose: L-glucose and D- glucose, but only D-glucose is biologically active (Figure 18) [102]. Stereoisomers are isomeric molecules with the same molecular formula and sequence of bonded atoms, but differ only in 3-D orientation. The orientation of L-glucose (pretty much a mirror-image of D-glucose) disables it from being metabolized by cells via glycolysis; however, L-glucose is not entirely 
unnecessary [102]. While it is less sweet than D-glucose and it cannot be used as a source of energy, L-glucose can be converted into a low-calorie sweetener. In addition, L-glucose has been found to be a laxative and has been proposed as a colon-cleansing agent [104].

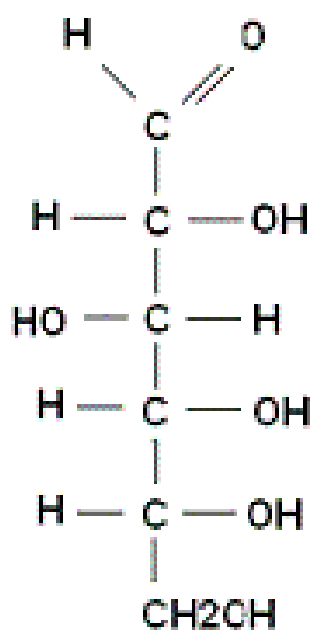

D-Glucose<smiles>O=CC(O)C(O)C(O)C(O)C(O)CO</smiles>

L-Glucose

Figure 18 - D-glucose and L-glucose are two stereoisomers of the aldohexose sugars. However, D-glucose is the biologically active form (Permission to reprint by [102]).

\subsubsection{Normal Blood Glucose Levels}

Most mammals normally maintain a blood glucose level within the range of $3.6 \mathrm{mmol} / \mathrm{L}$ and $5.8 \mathrm{mmol} / \mathrm{L}$ (about $80 \mathrm{mg} / \mathrm{dL}$ to $120 \mathrm{mg} / \mathrm{dL}$ ). The average normal blood glucose level in a human is $5 \mathrm{mmol} / \mathrm{L}$ (approximately $90 \mathrm{mg} / \mathrm{dL}$ ). Thus, the total amount of glucose circulating in blood, assuming the blood volume in an adult human is $5 \mathrm{~L}$, is about 3.3 to 7.0 grams [105]. According to the American Diabetes Association, after a person consumes an average size meal, the post-meal glucose levels for a non-diabetic person usually reach $140 \mathrm{mg} / \mathrm{dL}(7-8 \mathrm{mmol} / \mathrm{L})$ [88]. Diabetics, however, have a pre-meal glucose level of $90 \mathrm{mg} / \mathrm{dL}-130 \mathrm{mg} / \mathrm{dL}$ and can reach a post-meal glucose level of $180 \mathrm{mg} / \mathrm{dL}$ [106]. 
While this may seem like a substantial difference to some, it is actually quite surprising that such a small amount of glucose is circulating in the blood. Assuming that the average adult male is $165 \mathrm{lbs}$. ( $75 \mathrm{~kg})$ and contains a blood volume of $5 \mathrm{~L}$ (1.3 gal), a normal blood glucose level of $100 \mathrm{mg} / \mathrm{dL}(5.5 \mathrm{mmol} / \mathrm{L})$ is equivalent to only $5 \mathrm{~g}$ of glucose in the entire volume of blood, and about $45 \mathrm{~g}$ in the total volume of body water [105]! To visualize this, 5 grams of sugar is about the mass of a small packet of Splenda sugar. It is astonishing that such an insignificant amount of glucose can differentiate a healthy patient from a diabetic patient.

Knowing the various blood sugar ranges is useful when diagnosing diabetes mellitus. A type I diabetic will usually have very low plasma insulin levels during fasting and after a meal. This is contrary to type II diabetics who have abnormally high levels of plasma insulin during fasting and after a meal. This level increases even more after ingesting a "load" of glucose during a glucose tolerance test [107]. A glucose tolerance test helps establish the diagnosis of diabetes and also the type. As seen in the "glucose tolerance curve" (Figure 19), when a normal fasting person ingests 1 gram of glucose per kilogram of body weight, the blood glucose level increases from about $90-100 \mathrm{mg} / \mathrm{dL}$ to about $120-140 \mathrm{mg} / \mathrm{dL}$, and falls back to its normal values after 2 hours [107]. Recall that in a person with diabetes, the starting fasting blood glucose level is usually around $120-130 \mathrm{mg} / \mathrm{dL}$ (it can even be above $140 \mathrm{mg} / \mathrm{dL}$ depending on the severity). After ingesting 1 gram of glucose, these patients exhibit a much greater rise in blood glucose concentration and take about 4-6 hours to return to normal levels, sometimes not even reaching normal levels and never dipping below it [107]. The slow fall of the curve and the failure to fall below healthy glucose levels demonstrates that either normal insulin secretion after glucose ingestion does not occur, or there is a decreased sensitivity to insulin. Distinguishing between type I and type II diabetes in these patients comes down to analyzing the plasma insulin levels - 
again, with plasma insulin being low or undetectable in type I diabetics, and high in type II diabetics.

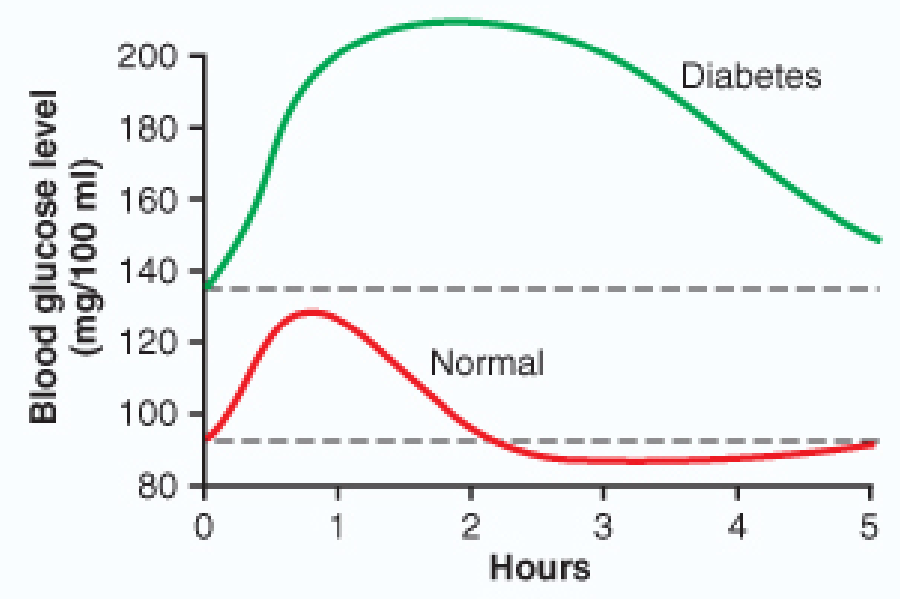

\section{(C) Elsevier. Guyton \& Hall: Textbook of Medical Physiology 11e - www.studentconsult.com}

Figure 19 - A glucose tolerance test illustrates that a normal patient who has consumed glucose will return to homeostasis after about 2 hours. A diabetic patient, however, will take 4-6 hours and sometimes not even reach normal glycemic levels. Distinguishing between type I and type II diabetes comes down to analyzing the plasma insulin levels - low levels in type I diabetics, and high levels in type II diabetics (Permission to reprint by [107]).

\subsubsection{Metabolism \& Regulation of Glucose}

Most of the mechanics and characteristics of the human body's physiologic functions are based on one common goal - achieving and maintaining homeostasis. Homeostasis is a property of an open or closed system, in this case the human body, which regulates its internal environment and tries to maintain a stable, constant condition. Vitals such as blood pressure, heart rate, calcium-release, hormonal secretions, and especially blood glucose levels try to achieve and maintain a homeostatic state. When it comes to maintaining a stable blood glucose level, carbohydrate metabolism with the help of insulin plays a key role. The final products of 
carbohydrate digestion are usually glucose, fructose, and galactose - glucose usually representing about $80 \%$ of the end products [108]. After absorption through the intestinal tract, most of the fructose and almost all of the galactose is converted to glucose in the liver; thus, leaving little fructose and galactose circulating in the blood [108]. Therefore, more than 95\% of all monosaccharides circulating in the blood is glucose, and when carbohydrates transport through cell membranes, it is common for carbohydrates to convert into glucose first [109].

The maximum molecular weight for a particle to diffuse readily through a cell membrane is usually 100 atomic mass units (amu). Although glucose has a molecular weight of $180 \mathrm{amu}$, it still passes into the interior of cells with a reasonable degree of freedom via facilitated diffusion $[109,110]$. Facilitated diffusion is the transport of a particle, in this case glucose, with the help of a carrier enzyme. Penetrating through the lipid matrix of the cell membrane are large numbers of protein carrier molecules that bind with glucose, cause glucose to undergo a conformational change, and easily transport the newly structured glucose to the other side of the cell membrane. This process is usually initiated by an existing concentration gradient that promotes more glucose to follow into the cell [110].

Insulin drastically increases the rate of glucose transport - nearly ten times more than the rate of transport when insulin is not present. To initiate the process of glucose consumption on a target cell, insulin first binds to the $\alpha$-subunits of its cell membrane receptor protein outside of the cell, which causes the autophosphorylization of the receptor's $\beta$-subunits inside the cell; thus, making the insulin receptor protein an enzyme-linked receptor[107] (Figure 20). Enzyme-linked receptors are proteins that pass through the cell membrane only once and have a hormone binding site on the outside of cell and the enzyme-binding site inside the cell [110]. Once activated by the hormone binding to the receptor, the enzyme inside the cell is immediately 
activated. The autophosphorylization of the $\beta$-subunits activates tyrosine kinase, which causes the phosphorylation of insulin-receptor substrates (IRS) [107]. IRS control glucose metabolism cause glucose transporters to be moved to the cell membrane in order to facilitate glucose entry into the cell [107].

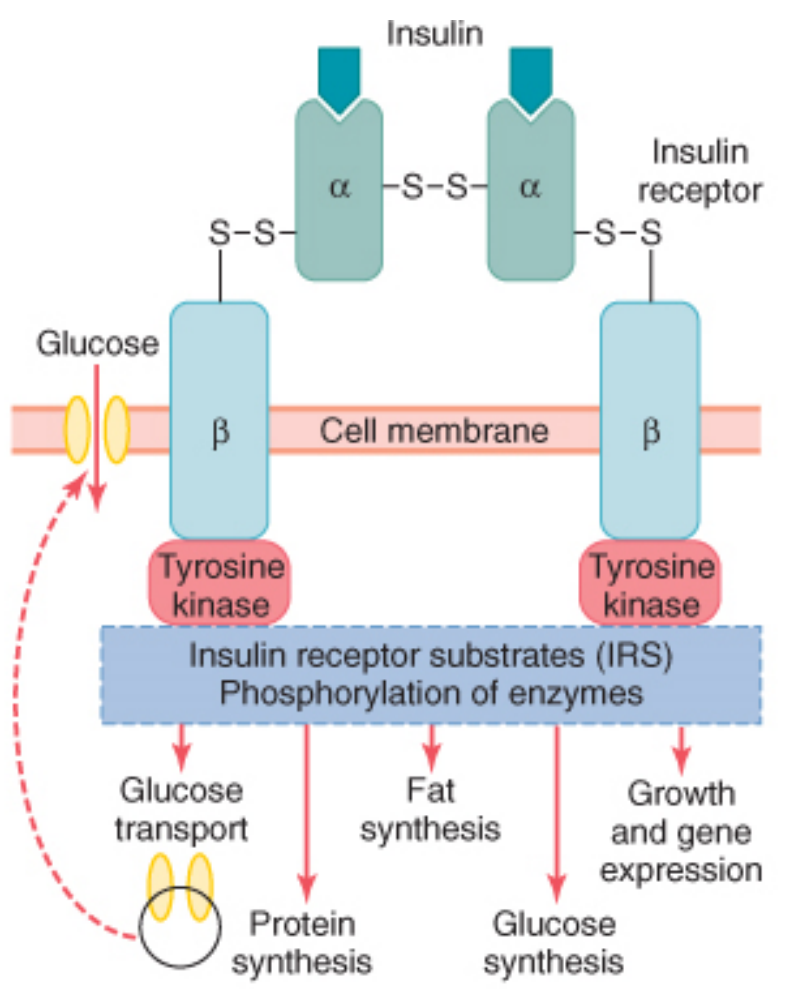

(C) Elsevier. Guyton \& Hall: Textbook of Medical Physiology 11e - www.studentconsult.com

Figure 20 - The binding of insulin to its cell membrane receptor induces a series of subsequent events, which cause the facilitated diffusion of glucose into the cell (Permission to reprint from [107]).

Insulin secretion is associated with energy abundance, that is, when there is a great abundance of energy-giving foods in a person's diet (high carbohydrate diet), large quantities of insulin are secreted due to the high concentration of blood glucose [111]. Therefore, carbohydrate metabolism is a significant factor for diabetes. Insulin also plays a key role in storing excess energy in liver cells in the form of glycogen - a large polymer of glucose through the process of glycogenesis. Between meals, when food is not available and blood 
glucose concentration begins to fall, insulin secretion decreases rapidly and the glycogen in the liver is converted back into glucose and secreted back into the blood to maintain homeostasis. As blood glucose concentrations rise above $100 \mathrm{mg} / \mathrm{dL}$, the rate of insulin secretion increases rapidly [107] (Figure 21).

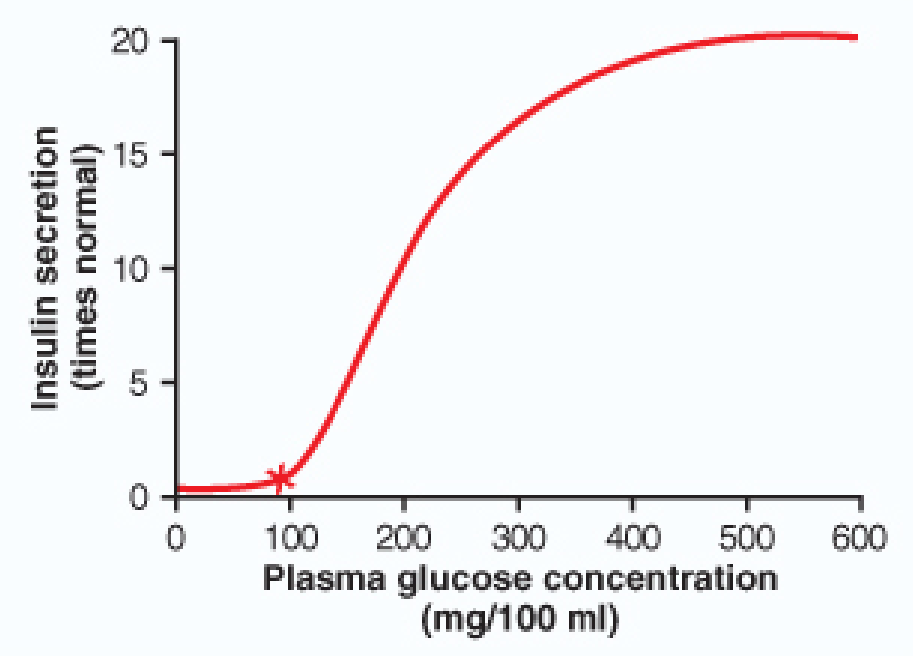

\section{(C) Elsevier. Guyton \& Hall: Textbook of Medical Physiology 11e - www.studentconsult.com}

Figure 21 - As blood glucose concentration rises, the rate of insulin secretion rises at a rapid rate. This increase in insulin secretion is non-existent in type I diabetics, and ineffective in type II diabetics - thus, resulting in hyperglycemia (Permission to reprint by [107]).

The rate of insulin secretion can reach nearly 20-25 times the basal level at extremely high blood glucose concentrations. The cessation of insulin secretion is almost equally as rapid, occurring within 3-5 minutes after blood glucose levels return back to the normal fasting level [107]. This association is an important feedback mechanism for regulating blood glucose concentration; however, in type I diabetics, this feedback mechanism is non-existent [112]. While this mechanism is present in type II diabetics, a dysfunction in the insulin-signaling cascade results in insulin resistance. 


\subsubsection{Dysfunction of Glucose Metabolism \& Insulin Signaling}

The dysfunction in the mechanism of insulin secretion in diabetic patients starts with the destruction of pancreatic $\beta$-cells. The $\beta$-cells have a large number of glucose transporters, GLUT-2 and GLUT-4 for example, that permit a rate of glucose influx that is correlated to the blood glucose concentration [113]. Glucose is then phosphorylated inside the B-cells into glucose-6-phosphate by an enzyme called glucokinase. This step senses and adjusts the amount of insulin that will be secreted. Glucose-6-phosphate is oxidized to form adenosine triphosphate (ATP), which inhibits ATP-sensitive potassium channels. This inhibition of potassium entering the cell causes drastic depolarization, which in turn, activates voltage-gated calcium channels. The entrance of calcium stimulates vesicles, containing insulin, to fuse with the cell membrane, and secrete insulin into the blood via exocytosis [114] (Figure 22).

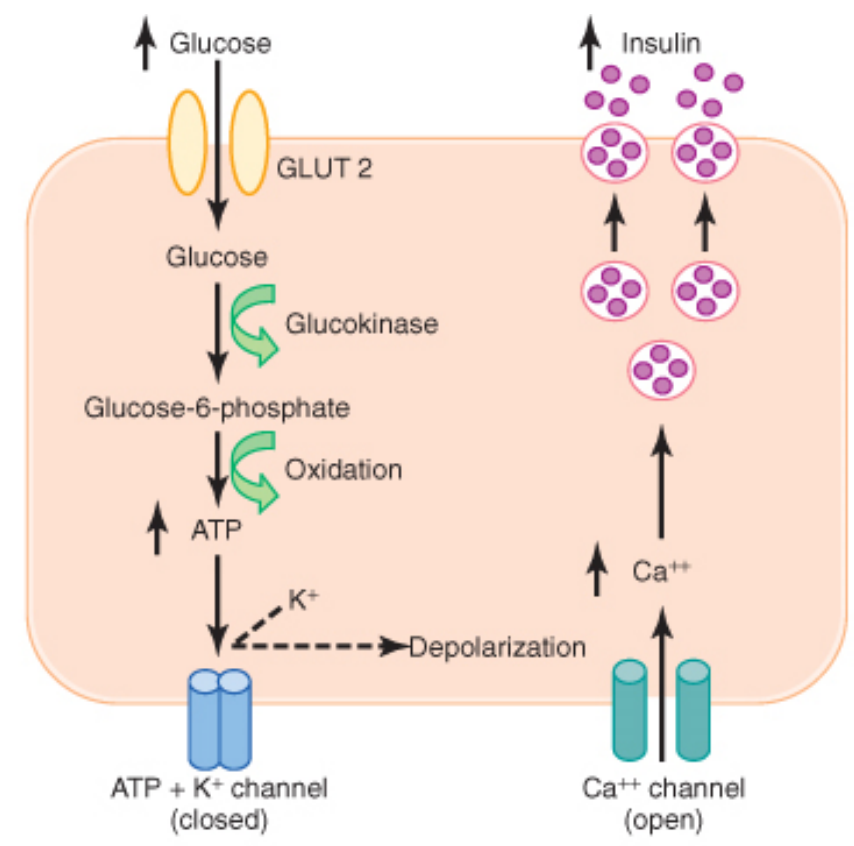

(C) Elsevier. Guyton \& Hall: Textbook of Medical Physiology 11e - www.studentconsult.com

Figure 22 - The influx of glucose into the pancreatic $\beta$-cells is controlled by glucose transporters (GLUT-2 and GLUT-4) depending on the blood glucose concentration (Permission to reprint from [107]. 
With the destruction of $\beta$-cells in type I diabetics, there is a lack of glucose transporters and glucokinase to phosphorylate the glucose into glucose-6-phosphate; thus, resulting in a lack of insulin secretion to compensate for the increase in blood glucose concentration.

Insulin resistance in type II diabetes is caused by abnormalities of the signaling pathway that link the activation of the cell membrane insulin receptor with the multiple subsequent cellular mechanisms that result in glucose uptake [115]. Although many of the proximal steps in insulin signaling have been identified [116], the downstream pathways that are important for insulin to maintain glucose homeostasis are less well defined [117]. Different IRSs are expressed in different tissues, but produce the desired effects on insulin signaling [107, 115]. Some animal studies associated insulin resistance with the defects and inactivity of phosphoinositide 3-kinase - an enzyme responsible for intracellular trafficking - resulting in decreased activation of glucose transport proteins $[117,118]$. Other animal studies claim that the defect in signaling occurs between the insulin receptors and the phosphorylation of IRS and other enzymes within the cell [119]. The exact dysfunction in the signaling cascade is still under investigation, but proposed mechanisms, if correct, offer new therapeutic targets for insulinsensitizing agents, especially for type II diabetics.

\subsubsection{Summary}

Hyperglycemia is the major consequence of diabetes since it is the root cause of so many complications in diabetic patients. Hyperglycemia is characterized as high concentration of blood glucose. The aim of this thesis is to create a diabetic BVM, and from the research that was conducted, the most practical method of inducing a diabetic environment was to induce a state of hyperglycemia. Therefore, the first set of experiments conducted and explained in this chapter 
involved the creation of a hyperglycemic environment, which was hypothesized as a high glucose cell media solution. In addition, an established method of monitoring the glycemic cell media solutions was necessary. Thus, the goals of the studies in this chapter were:

1. To explore and identify practical methods of measuring levels of glucose in different glycemic cell media solutions;

2. To develop and establish a procedure to create hyperglycemic cell media solutions with the addition of glucose into base cell media; and

3. To use the hyperglycemic cell media solutions in order to evaluate the different methods of monitoring glucose concentrations and determine the most feasible method.

The following section begins by describing various methods available for measuring blood glucose concentrations, followed by methods of creating different hyperglycemic solutions to test the reliability of various glucose monitoring methods.

\subsection{Methods}

There are a variety of methods to measure blood glucose concentrations, ranging from bio-implants and continuous monitoring systems, to blood glucose meters and visual blood glucose test strips. Since the glycemic solutions in these experiments would be produced using cell culture media instead of blood, it was essential to assess not only the feasibility of each method, but also the method that would provide the most accurate and precise results in cell culture solutions. A range of methods was first reviewed, as will be summarized below, and then the top two methods were selected for experimental testing. 


\subsubsection{Methods of Measuring and Monitoring Blood Glucose}

In this section, three methods for measuring blood glucose are described: blood glucose meters, continuous glucose monitoring systems, and visual blood glucose strips. In addition to these methods, a look into future methods of monitoring, such as glucose sensing bio-implants, and other non-invasive technologies, are briefly discussed.

\subsubsection{Blood Glucose Meter}

A blood glucose meter is a medical device used to measure blood glucose concentrations. The meter requires only a small drop of blood $(3 \mu \mathrm{L}-10 \mu \mathrm{L})$ that is obtained by pricking the tip of a finger with a small lancet, and a disposable test strip consumes the drop of blood, via capillary action and measures the blood glucose level in units of $\mathrm{mg} / \mathrm{dL}$ or $\mathrm{mmol} / \mathrm{L}$. This device is a key addition to "home blood glucose monitoring" and helps patients avoid hyperglycemic/hypoglycemic episodes by testing several times a day.

The blood glucose meter functions via the oxidation of glucose to gluconolactone that is catalyzed by glucose oxidase (GOD), or some meters use a similar reaction catalyzed by glucose dehydrogenase (GDH) instead [120]. Most meters use an electrochemical method with the mentioned reagents. The disposable test strips contain a capillary that sucks up the drop of blood and the glucose within the blood reacts with an enzyme electrode containing either GOD or GDH [121]. The enzyme is reoxidized with an excess of ferricyanide ion, a mediator reagent, and this mediator in turn is reoxidized by the reaction at the electrode that generates an electric current. The total charge that passes through the electrode is proportional to glucose concentration in the drop of blood that reacted with the electrode [122]. This method is an 
example of the coulometric method - very much equivalent to determining how hard a ball was thrown by measuring the distance it traveled in a certain amount of time.

The first two models of the blood glucose meters to dominate North America were the Glucometer (owned by Bayer) and the Accu-Chek (by Roche, previously known as Boehirnger Mannheim) (Figure 23) [123]. Compared to visual glucose test strips, which were more popular before the arrival of the blood glucose meter, blood glucose meters are preferred by patients mostly for the ease of use. However, the accuracy of glucose meters is a common clinical concern $[123,124]$.

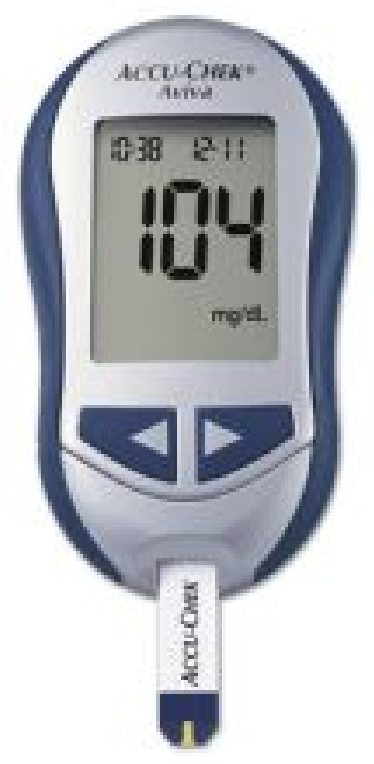

Figure 23 - The Aviva Accu-Chek blood glucose meter is the most popular meter used among diabetics to test blood sugar levels (Permission to reprint by Accu-Chek Blood Glucose Monitoring Systems).

Most blood glucose meters have the same level of accuracy (+/- 10\%-15\%); however, factors such as calibration of the meter, ambient temperature, size and quality of the blood sample, contamination of the meter, humidity, and aging of the associated test strips can affect 
the accuracy of each test [125]. To limit the inaccuracy of test results, the Clarke Error Grid Analysis (EGA) (Figure 24) was a common way of analyzing and displaying accuracy of readings [126]. The EGA was developed in 1987 to quantify the clinical accuracy of a patient's estimate of their blood glucose levels compared to the value obtained on the meter [127]. The EGA became accepted as the key reference for determining the accuracy of blood glucose meters. The grid was broken down as a scatter plot comparing a reference glucose meter and an evaluated glucose meter into five regions:

Region A - values within $20 \%$ of the reference glucose meter

Region B - values outside of $20 \%$ but do not lead to inappropriate treatment

Region C - values leading to unnecessary treatment

Region D - values indicating potentially dangerous failure to detect glycemic imbalance Region E - values that confuse treatment of hypoglycemia or hyperglycemia 


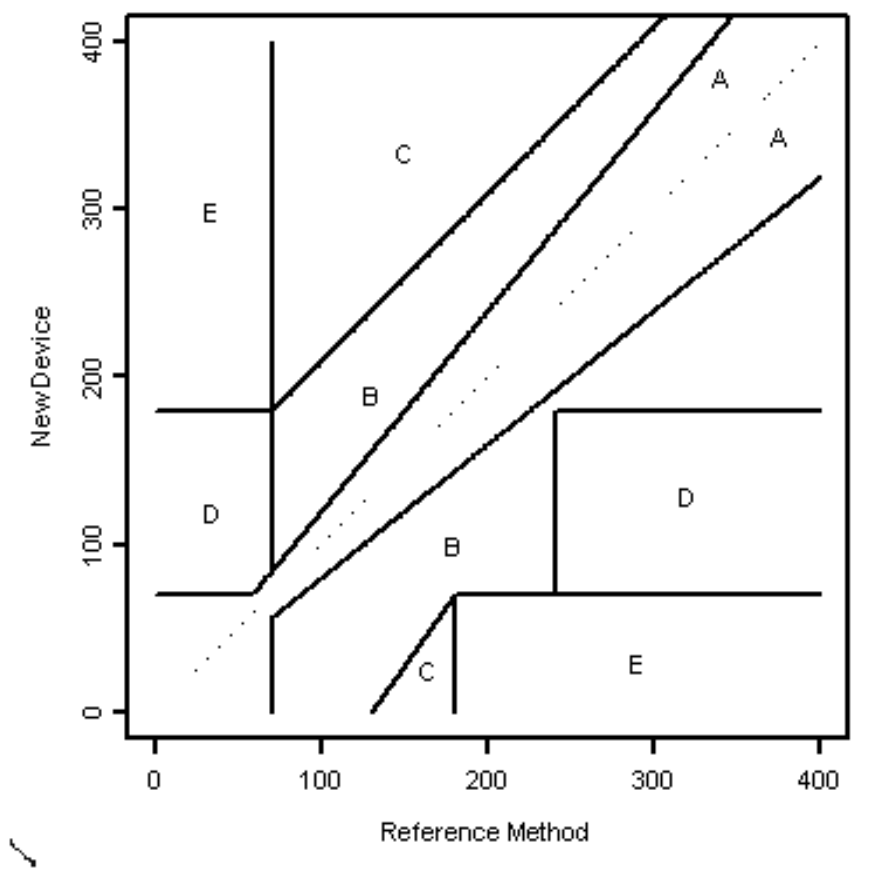

Figure 24 - The Clarke Error Grid Analysis (EGA) was developed to quantify the clinical accuracy of a patient's estimate of their blood glucose compared to the value obtained on the meter. Patients could estimate their blood glucose levels by another blood glucose meter or visual test strips (Permission to reprint by [127]).

The analysis takes into account the value of the evaluated blood glucose meter, the value of the reference blood glucose meter, the relative difference between the two values, and the clinical significance of the difference [127]. Although there is a level of inaccuracy in blood glucose meters, animal and clinical studies monitoring diabetic patients use multiple blood glucose meters to ensure that an accurate blood glucose level is being measured [128].

\subsubsection{Continuous Glucose Monitoring}

A continuous glucose monitoring system (CGMS) is a device that monitors and records blood glucose concentrations twenty-four hours a day (Figure 25). The idea behind this device is to measure blood sugars continuously for 3-5 days while the diabetic patient conducts daily routines and activities. The "sensor" of the CGMS is inserted subcutaneously just under the skin 
of the abdomen where it measures glucose levels in the tissue every 10 seconds. Information is transmitted from the sensor to the associated insulin pump that reads and plots the changes in glucose concentration. The entire system usually records an average glucose value every 5 minutes and plots it on a curve for the patient to see. While standard blood glucose meter measurements are still taken and entered into the insulin pump for calibration, the CGMS continuously measures the glucose concentrations during those critical times, such as before and after a meal, during and after exercise, and during the night when the patient is asleep. After the 3-5 day period, the sensor is removed and stored information in the insulin pump is downloaded to a computer where the plots of recorded glucose concentrations are printed in order to evaluate fluctuations and patterns.

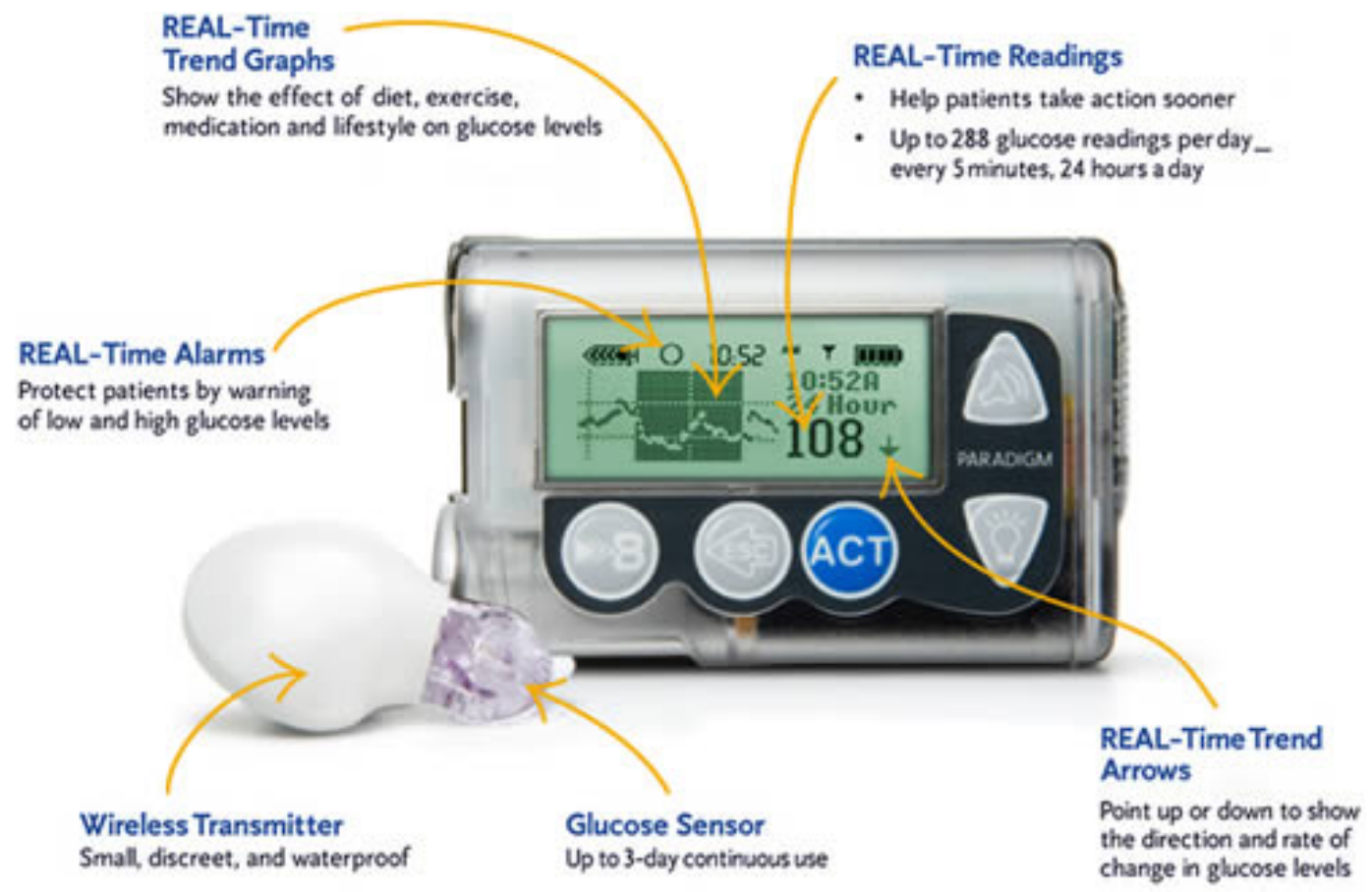

Figure 25 - The MiniMed Paradigm REAL-Time System uses a glucose sensor attached to a wireless transmitter that records blood glucose levels and graphs it on the incorporated insulin pump (Permission to reprint by Medtronic Diabetes). 
This system is not intended for long-term self-care or as a replacement for the blood glucose meter; the CGMS is mostly used to study patterns of a patient's glucose levels, especially at those critical times mentioned above. For example, a pattern or trend can indicate that a patient's blood glucose level is dangerously low during the night and is at risk of a hypoglycemic episode while fast asleep. Or, a pattern revealing an elevated blood glucose level during the afternoon can expose a patient's tendency to eat a high carbohydrate lunch at work. Though studies have shown that with the information given from the CGMS, patient HA1c levels have decreased with adjustments to their therapies, the CGMS still cannot replace frequent testing with blood glucose meters [129]. Animal tests are continuously conducted to ensure CGMS are accurately monitoring glucose concentration. Testing on diabetic cats showed that there was a good correlation between the CGMS values and blood glucose concentration measured using a blood glucose meter [130]. Numerous clinical studies have shown that using CGMS provides adequate information, and treatment changes would not have been made on the basis of glucose meter data alone. The improvement of a patient's health, however, is only achieved if the information supplied by the system is used properly and effectively towards improved insulin therapy $[131,132]$.

There are three main CGMS manufacturers - Medtronic, DexCom (Animus/J\&J), and Abbott. Medtronic currently has two FDA-approved CGM systems in the market - the Guardian REAL-Time CGMS, and the MiniMed Paradigm REAL-Time System. DexCom has the DexCom Seven, and Abbott has the FreeStyle Navigator. Though these systems are produced by different manufactures, the overall concepts among the products are very similar with no significant advantage of one over another. 


\subsubsection{Visual Blood Glucose Test Strips}

Visual blood glucose test strips are a convenient and simple alternative for diabetic patients who occasionally monitor blood glucose levels (usually type II diabetic patients) and patients who cannot afford the disposable test strips used for blood glucose meters. Visual blood glucose test strips that change color (not to be confused with disposable test strips that are associated with the blood glucose meter) were widely used in the 1980s and had a slight advantage over the blood glucose meter since they could be cut longitudinally to save money. However, once the accuracy and insurance coverage improved for the blood glucose meter, visual blood glucose test strips lost popularity [124].

There are various types of visual blood glucose strips, usually taking measurements using blood or urine. Blood glucose dipsticks react with blood glucose and display a color that correlates with a specific glucose concentration. Unfortunately, urine glucose dipsticks cannot reflect simultaneous blood glucose concentrations but do have incredible reproducibility and can answer the question of whether or not a stable glucose concentration is being tested.

The type of visual blood glucose strip this chapter will focus on is the Betacheck Glucoflex-R that uses a color comparison for accurate and clear readings with only a drop of blood (10-20 $\mu \mathrm{L})$ from the fingertip placed on the droplet zone of the test strip. The test zone of the Glucoflex-R consists of two test pads that undergo a reaction with glucose via the glucose oxidase/peroxidase reaction. This reaction causes a color change in direct proportion to the amount of glucose in the blood. Typically, visual blood glucose test strips (including the Glucoflex-R) will have approximately ten color assortments that correlate with glucose levels from $20 \mathrm{mg} / \mathrm{dL}$ all the way to $800 \mathrm{mg} / \mathrm{dL}$. 
One advantage of visual blood glucose strips over blood glucose meters is accuracy. Glucose meters have to go through constant maintenance and calibration to ensure accurate readings. The accuracy of chemical visual test strips (+/-5\%) outdoes the accuracy of blood glucose meters (+/- 10\%-15\%). The quality check for Glucoflex-R visual strips, however, only involves comparing a new test strip against the " 0 " color reference on the container.

The most obvious limitation of these test strips, however, is the limited quantitative result that is given along with the potential variance of the results. While blood glucose meters are able to give an exact measurement, visual glucose strips can either give a range of values or an estimation of a close value. In a comparative study, for blood glucose values less than or equal to $180 \mathrm{mg} / \mathrm{dL}$, blood glucose meters had the best correlation. For glucose values of 181-400 $\mathrm{mg} / \mathrm{dL}$, visual strips were just as accurate as meters, but not as precise [133].

\subsubsection{Glucose sensing bio-implants}

To ease the burden of continuous blood glucose monitoring on patients, the development of long-lasting glucose sensing bio-implants is under development.

One particular product under development is the SMSI Glucose Sensor. The sensor is implanted under the skin in a short outpatient procedure and is designed to automatically measure interstitial glucose every few minutes. With a small external reader, the sensor will communicate wirelessly allowing the user to monitor levels on demand [134]. The implanted sensor's targeted operational life is 6-12 months, after which it would be replaced [135]. Currently, this product is in the process of completing animal studies; however, results and conclusions are not yet available for public viewing. 


\subsubsection{Non-Invasive Technologies}

One major burden diabetics continuously complain about is the constant finger pricking that is necessary for blood glucose testing. Manufacturers of diabetic devices are researching and developing new technologies that will not require direct contact with blood to measure glucose levels. These new non-invasive technologies range from near IR detection, to ultrasound, to dielectric spectroscopy $[136,137]$.

Although a thorough review of these emerging technologies is beyond the scope of this thesis, one intriguing product under development is the Sensy Glucose Tracking System. Sensy Medical is developing the new method of glucose monitoring using near-infrared (NIR) diffuse reflectance spectroscopy (DRS) to measure glucose levels in a non-invasive manner [138]. NIR light ranges from 750-2500nm and interacts differently with various molecules (fat, glucose, protein, water, etc.) by absorbing different amounts of NIR light. The amount of light absorbed is proportional to the concentration of the particular molecule [138]. Thus, the blood glucose concentration will be measured in light absorbance units that can be converted to the usual blood glucose units of $\mathrm{mg} / \mathrm{dL}$.

\subsubsection{Summary}

After researching and discussing the different practical methods available to measure blood glucose concentrations, the next step was to determine the most feasible method(s) for the laboratory and apply that method of measurement to the measurements of various glycemic solutions. Based on the information from the above section, the most feasible methods to monitor the glycemic solutions were the blood glucose meter and visual blood glucose test strips, 
mostly due to their quick return of results, availability, and affordability. Thus, the next step in the study was to develop the procedure to create cell media solutions of various glucose concentrations, in hopes that a solution with elevated glucose concentrations properly replicates a hyperglycemic environment, and can be accurately measured by either the blood glucose meter or the visual blood glucose test strips.

The following section discusses the experimentation conducted to develop and establish various cell media solutions of specific glucose concentrations, and whether the tested methods of measuring these glycemic solutions - the blood glucose meter and visual blood glucose test strips - accurately and precisely measured the expected glucose concentrations.

\subsubsection{Methods of Producing and Monitoring Different Glycemic Solutions}

\subsubsection{Levels of Glycemia}

The first step taken when developing the proper procedure for creating glycemic cell media solutions was to determine what glucose concentrations to achieve. Based on the discussion regarding the different levels of hyperglycemia seen in diabetics, three different glucose concentrations were specified, created, and monitored over time. The different glucose concentrations were organized as follows:

Normal glycemic level: $100 \mathrm{mg} / \mathrm{dL}$

Hyperglycemic level: $250 \mathrm{mg} / \mathrm{dL}$

Extreme Hyperglycemic level: $400 \mathrm{mg} / \mathrm{dL}$ 
Four levels of hyperglycemia were initially attempted, but after preliminary testing, it was difficult to maintain a degree of separation among the three glycemic levels without crossing over into another level of hyperglycemia (see Appendix A for summary of preliminary testing). Recall that only 5 grams of glucose are within the entire volume of blood in an average human male (5 L); thus, there is an extremely small margin of error when adding glucose to small volumes of cell culture media.

\subsubsection{Cell Media with D-(+)-Glucose}

To create the different glycemic cell media solutions, Sigma G6152 D-(+)-glucose (Figure 26) was added to Gibco Medium 199 (+: Earle's salts, L-Glutamine, 2.2 g/L Sodium bicarbonate) (Figure 27) by Invitrogen. An important note to consider is that cell media does not contain the same properties as blood. Blood contains many constituents (plasma, albumin, hemoglobin, uric acid, etc.); thus, the viscosity of blood is much greater than the viscosity of just cell media with added glucose. In addition, blood contains functioning and circulating cells that are not present in cell media; however, for the purposes of creating an in vitro environment, the use of cell culture media was appropriate. 


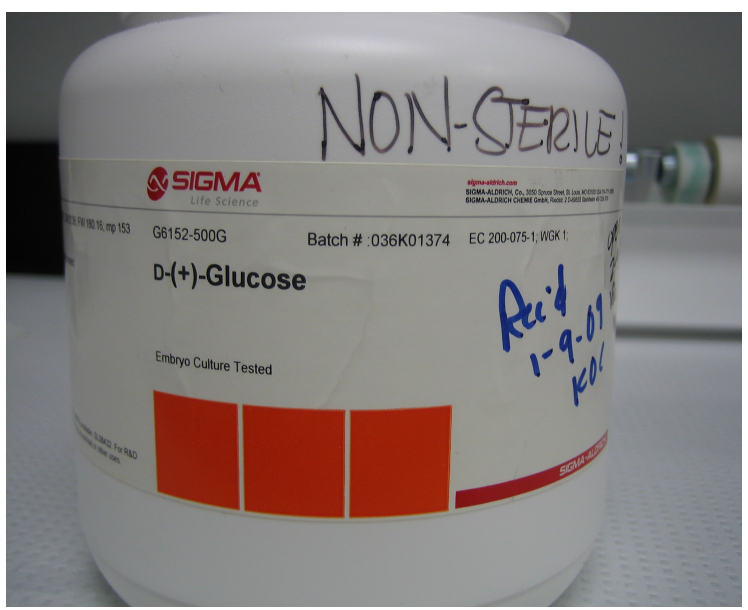

Figure 26 - Sigma G6512 D-(+)-Glucose was added to Gibco Medium 199 in the attempt to create glycemic cell media solutions.

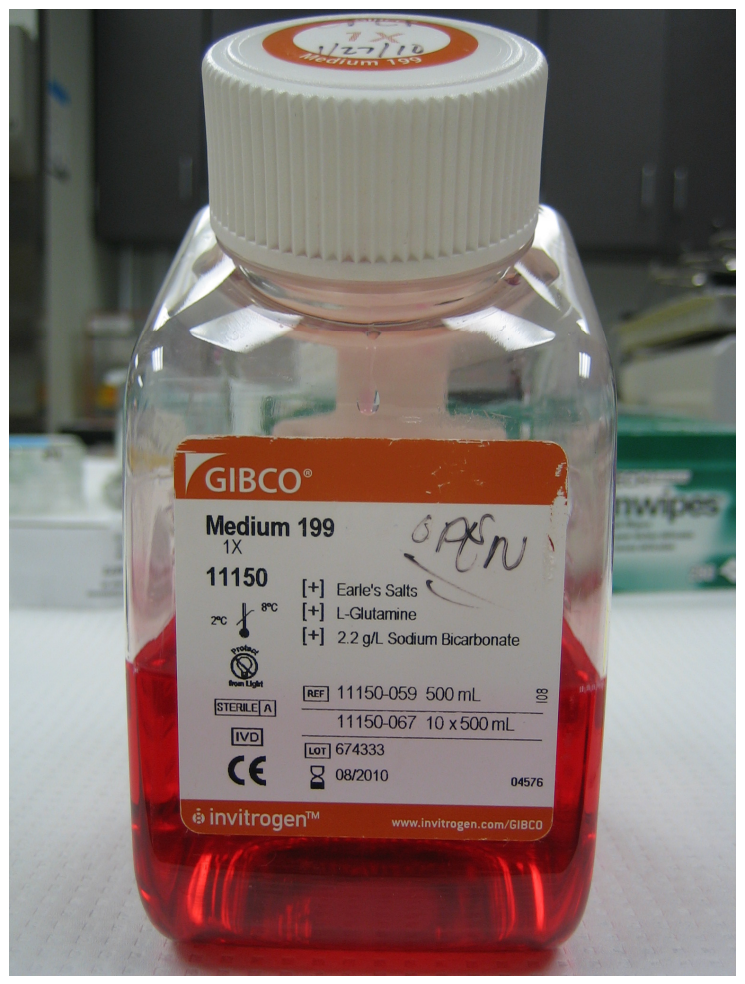

Figure 27 - Gibco Medium 199 by Invitrogen was used as the base cell media for the experimentation of different glycemic cell media solutions. This media was also used for later experimentation when developing glycemic cell media solutions for use with human umbilical vein endothelial cells (HUVECs).

Three separate solutions were created for each level of glycemia. In order to produce the different solutions, the following recipes were used: 
- For the $100 \mathrm{mg} / \mathrm{dL}$ normal glycemic solution:

$0.100 \mathrm{~g}$ of Sigma G6152 D-(+)-Glucose was weighed and added to 100 $\mathrm{mL}$ of Gibco Medium 199 in a $150 \mathrm{~mL}$ beaker

- For a $250 \mathrm{mg} / \mathrm{dL}$ hyperglycemic solution:

$0.250 \mathrm{~g}$ of Sigma G6152 D-(+)-Glucose was weighed and added to 100 $\mathrm{mL}$ of Gibco Medium 199 in a $150 \mathrm{~mL}$ beaker

- For a $400 \mathrm{mg} / \mathrm{dL}$ extremely hyperglycemic solution:

$0.400 \mathrm{~g}$ of G6152 D-(+)-Glucose was weighed and added to $100 \mathrm{~mL}$ of Gibco Medium 199 in a $150 \mathrm{~mL}$ beaker

After the glucose was weighed and added to the cell media, each solution was placed on a stir plate with a magnetic stir bar, and stirred for 30 minutes at $25^{\circ} \mathrm{C}$ to promote uniform mixing.

\subsubsection{Monitoring Hyperglycemic Levels}

The blood glucose meter and the visual blood glucose test strips were the selected methods of measurement to be tested. For this experiment, the glucose concentration of the developed glycemic cell media solutions were then taken at $\mathrm{t}=1,2,3,4,5,6,12,24,36$, and 48 hours after mixing began.

Two blood glucose meters were used to monitor glucose concentration - the Aviva AccuChek blood glucose meter, and the Bayer Contour blood glucose meter. These meters were chosen because the meters and the associated test strips were easily obtainable. Also, from personal experience as a Medical Assistant in an Endocrinology office, these meters were the favorite among doctors and patients due to their accuracy and reliability. 
The type of visual blood glucose strips used was the Glucoflex-R Blood Glucose Strips (Figure 28). These strips were purchased from Ambe Medical Group in England and were at $\$ 16.11$ (9.99 GBP) for a set of 50 strips.

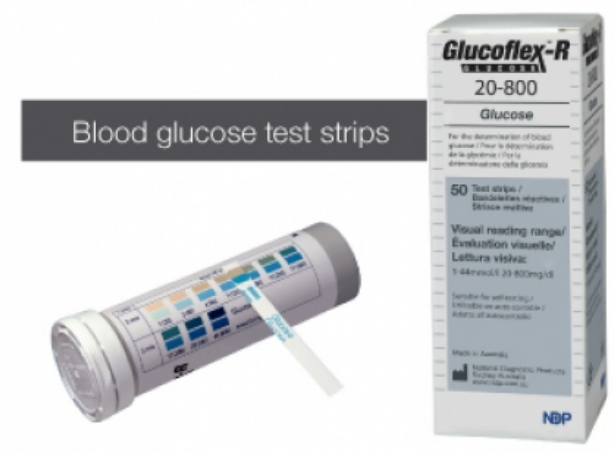

Figure 28 - Glucoflex-R Blood Glucose Strips by Ambe Medical Group in England (Permission to reprint by Ambe Medical Group).

Before taking measurements for each solution at the specific time point, both meters and the visual test strips were “calibrated” using Hyclone Dulbecco’s Modified Eagle Medium (DMEM) / High Glucose (+: 4.00 mM L-Glutamine, 4500 mg/L Glucose, Sodium pyruvate) $4500 \mathrm{mg} / \mathrm{L}$ high glucose cell media. This was manufactured high-glucose cell media that was already calibrated to accurately resemble a glucose concentration of $450 \mathrm{mg} / \mathrm{dL}$; thus, it was used to ensure each monitoring device was functioning properly.

The intention of using multiple blood glucose meters was to accumulate more data in hopes of obtaining normalized data, while also assessing which method was most consistent and appropriate for this application. The intention of the visual blood glucose strips was to ensure that the tested cell media was at least within the correct glucose range, and also confirm an 
accurate and stable glucose environment. Considering the levels of inaccuracy for both glucose meters and visual blood glucose strips, it was necessary to use both methods in order to identify any variance in the results. Plus, it would also expose whether or not one of the methods was significantly different from another.

After the glucose concentrations were recorded for the three solutions of each glycemic level, an average was taken along with the standard deviation, and a percent error was calculated based off of the targeted glucose level. Lastly, a series of two-tailed T-tests were conducted between each solution based on method of measurement, and an ANOVA test was conducted on all three solutions to ensure there was no statistical significant difference among the solutions within the same method of measurement. The following section discusses the accumulated results and analysis.

\subsection{Results}

\subsubsection{Results of Preliminary Testing}

Preliminary testing was conducted in order to establish the proper procedure of creating the different glycemic solutions. Once established, the glycemic cell media solutions were tested over a period of 48 hours in order to see which method of measurement was best for the glycemic solution study. The Bayer Contour meter and its test strips were unable to give consistent readings as an "error message" would continuously appear on the meter at random time intervals. Therefore, only the results from the Accu-Chek blood glucose meter and the Glucoflex-R visual blood glucose strips were used to draw conclusions. The following sections analyze and discuss the results accumulated for each method of measurement for each developed 
glycemic cell media solutions. However, one note must be mentioned before discussing the results, as it plays a significant role in the analysis of the results.

\subsubsection{Glycemic Level of Gibco Medium 199}

After testing, a significant attribution was discovered regarding the composition of the Gibco Medium 199. The recorded glucose concentrations were higher than the expected; thus, the chemical composition of the cell media was researched on the Invitrogen website. What was discovered was that the Gibco Medium 199 was a "low glucose" cell media that already contained of $1000 \mathrm{mg} / \mathrm{L}(100 \mathrm{mg} / \mathrm{dL})$ of D-glucose (Figure 29).

\begin{tabular}{|l|r|r|r|}
\hline \hline Thiamine hydrochloride & 337 & 4 & 0.0119 \\
\hline i-Inositol & 180 & 7.2 & 0.04 \\
\hline Inorganic Salts & 147 & 264 & 1.8 \\
\hline Calcium Chloride (CaCl2-2H2O) & 404 & 0.1 & 0.000248 \\
\hline Ferric Nitrate (Fe(NO3)3"9H20) & 246 & 200 & 0.813 \\
\hline Magnesium Sulfate (MgSO4-7H2O) & 75 & 400 & 5.33 \\
\hline Potassium Chloride (KCl) & 84 & 3700 & 44.05 \\
\hline Sodium Bicarbonate (NaHCO3) & 58 & 6400 & 110.34 \\
\hline Sodium Chloride (NaCl) & 156 & 141 & 0.904 \\
\hline Sodium Phosphate monobasic (NaH2PO4- & 110 & 110 & 1 \\
\hline 2H2O) & 180 & 1000 & 5.56 \\
\hline Other Components & 376.4 & 0.0399 \\
\hline D-Glucose (Dextrose) & & & \\
\hline Phenol Red & & 15 & \\
\hline Sodium Pyruvate & & & \\
\hline
\end{tabular}

Figure 29 - The Invitrogen website confirmed that the composition of the Gibco Medium 199 contained $1000 \mathrm{mg} / \mathrm{L}(100 \mathrm{mg} / \mathrm{dL})$ of D-glucose. 
To confirm, measurements were taken via the Accu-Chek blood glucose meter as well as the Glucoflex-R visual blood glucose strips, and both confirmed a glucose concentration of 100 $\mathrm{mg} / \mathrm{dL}$. Therefore, to compensate for the additional $100 \mathrm{mg} / \mathrm{dL}$ already within the cell media, the expected glucose concentrations for each solution were adjusted to the following levels:

Adjusted Normal glycemic level: $200 \mathrm{mg} / \mathrm{dL}$

Adjusted Hyperglycemic level: $350 \mathrm{mg} / \mathrm{dL}$

Adjusted Extreme Hyperglycemic level: $500 \mathrm{mg} / \mathrm{dL}$

Using the adjusted glucose concentrations, the accumulated data was analyzed.

\subsubsection{Normal Glycemic Solutions (200 mg/dL)}

A glycemic cell media solution of $200 \mathrm{mg} / \mathrm{dL}$ was developed to replicate a normal glycemic environment. Recall, three solutions composed of $0.100 \mathrm{~g}$ of Sigma G6152 D-(+)Glucose and $100 \mathrm{~mL}$ of Gibco Medium 199 were created. Three solutions gave an adequate data sample size for practical and repeatable data analysis. Table 3 displays the exact amounts of glucose added to each volume of cell media.

Table 3 - Amount of Sigma G6152 D-(+)-Glucose added to $100 \mathrm{~mL}$ of Gibco Medium 199 for 200 mg/dL Normal Glycemic Solutions

\begin{tabular}{|c|c|c|}
\hline Solution Sample & $\begin{array}{c}\text { Amount of Glucose Added } \\
(\mathrm{g})\end{array}$ & Volume of Cell Media (mL) \\
\hline $\mathbf{1}$ & 0.1009 & 100 \\
\hline $\mathbf{2}$ & 0.1003 & 100 \\
\hline $\mathbf{3}$ & 0.1004 & 100 \\
\hline
\end{tabular}


The purpose of the additional glucose was to compensate for any glucose lost during the transfer from the weigh boat to the beaker containing the cell media. After taking glucose concentrations at the specific time intervals for 48 hours, the accumulated results matched the expected glucose concentration of $200 \mathrm{mg} / \mathrm{dL}$.

Figure 30 is a plot showing the recorded glucose concentrations using the Aviva AccuChek blood glucose meter. This plot shows that the blood glucose meter recorded values very close to the expected concentration, and took approximately until $\mathrm{t}=2$ hours to reach the expected glucose concentration of $200 \mathrm{mg} / \mathrm{dL}$.

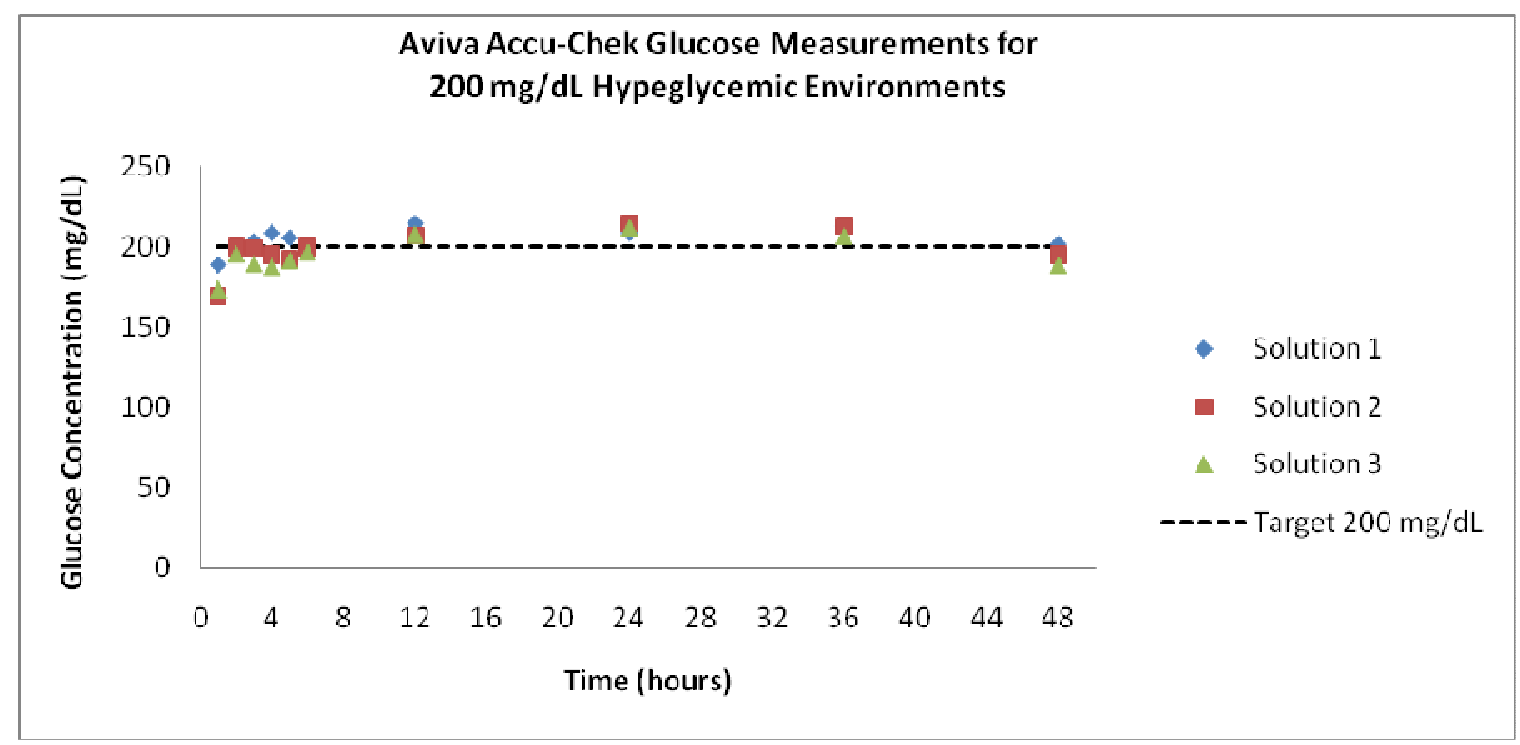

Figure 30 - The plot shows that the accumulated glucose concentrations by the Aviva AccuChek blood glucose meter matched the expected glucose concentration of $200 \mathrm{mg} / \mathrm{dL}$ for each solution. The glucose concentration rose rapidly, but plateaued at the expected value after roughly 2 hours of mixing.

As for the accumulated results by the Glucoflex-R visual blood glucose test strips, Figure 31 shows that, it too, was able to consistently measure a glucose concentration throughout the entire test, and the results were approximately equivalent to the expected $200 \mathrm{mg} / \mathrm{dL}$. However, the recorded measurements were not nearly as precise as those of the Aviva Accu-Chek blood 
glucose meter, as outliers within the Glucoflex-R measurements are obviously apparent. The Glucoflex-R visual strips also took until approximately $\mathrm{t}=2$ hours to record a consistent measurement of roughly $200 \mathrm{mg} / \mathrm{dL}$.

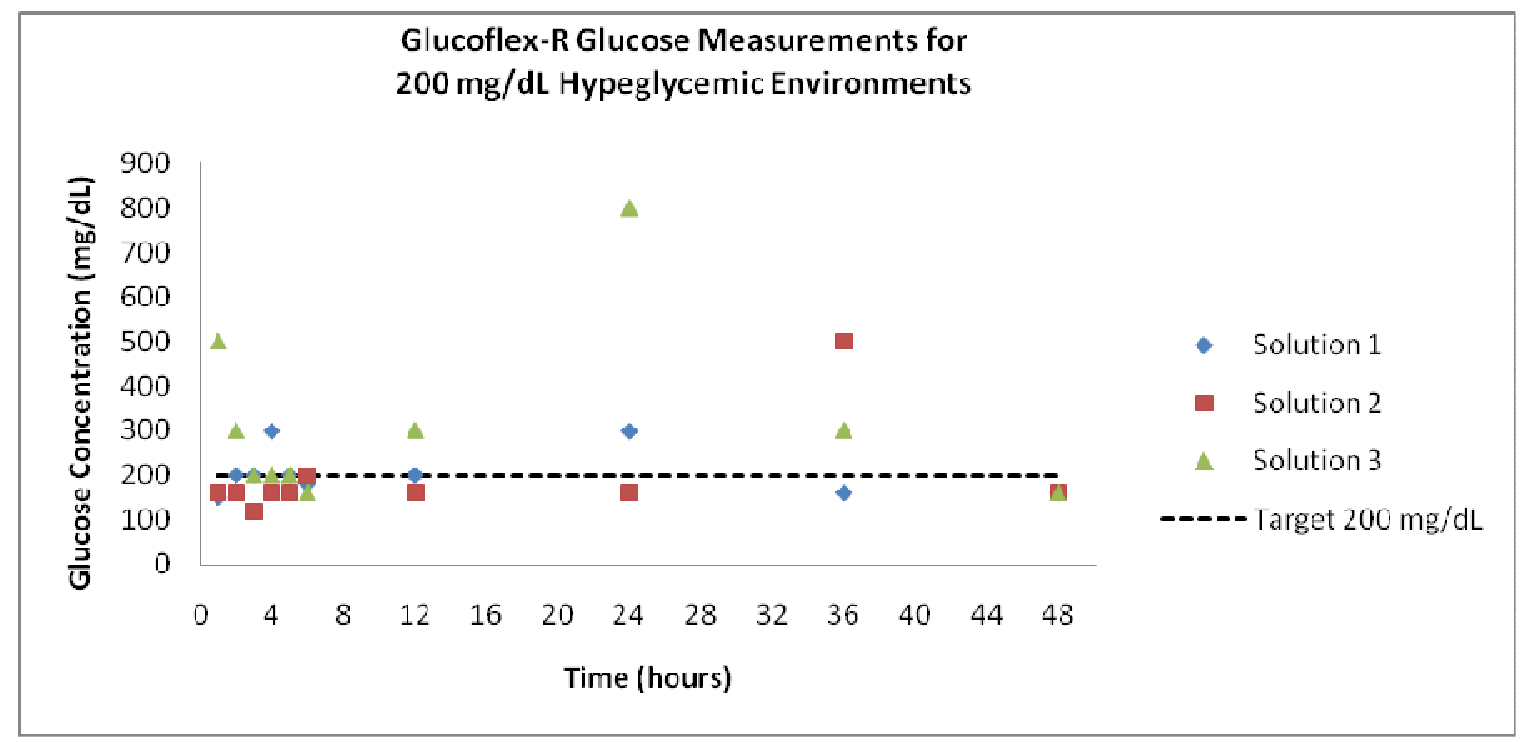

Figure 31 - The recorded glucose levels, using the Glucoflex-R visual test strips, were within the expected range of $200 \mathrm{mg} / \mathrm{dL}$. However, the variance of the recorded results was quite high, and is not an ideal characteristic for a sensitive hyperglycemic environment.

Error! Reference source not found.Table 4 shows the average glucose concentrations of each solution using the Aviva Accu-Chek blood glucose meter and the Glucoflex-R visual test strips, as well as the percent error in relation to the expected $200 \mathrm{mg} / \mathrm{dL}$ glucose concentration. Both methods of measurement had average glucose concentration close to the expected value (except for solution 3, which consisted of the outlier), considering they had low percent errors. However, the key factor to take into consideration is the low variance for the Aviva Accu-Chek blood glucose meter compared to the high variance for the Glucoflex-R visual test strips. It can be seen on Figure 30 and Figure 31 that the variance is much greater for the Glucoflex-R visual test strips compared to the Aviva Accu-Chek meter. This level of variance is not ideal for 
obtaining an accurate and precise glucose concentration, especially given the sensitivity of any hyperglycemic environment.

Table 4 - Average Glucose Concentrations Recorded with Accu-Chek Blood Glucose Meter \& Glucoflex-R Visual Blood Glucose Strips for 200 mg/dL Glycemic Solutions

\begin{tabular}{|c|c|c|c|c|}
\hline & Solution 1 & Solution 2 & Solution 3 & $\begin{array}{l}\text { Hyclone High Glucose } \\
450 \mathrm{mg} / \mathrm{dL} \text { Calibration }\end{array}$ \\
\hline $\begin{array}{c}\text { Average } \\
\text { Glucose } \\
\text { Concentration } \\
(\mathbf{m g} / \mathbf{d L})\end{array}$ & 204.70 & 198.50 & 195.30 & 446.56 \\
\hline $\begin{array}{l}\text { Standard } \\
\text { Deviation }\end{array}$ & 6.55 & 7.54 & 12.89 & 11.383 \\
\hline$\%$ Error & $2.35 \%$ & $-0.75 \%$ & $-2.35 \%$ & $-0.765 \%$ \\
\hline \multicolumn{5}{|c|}{ Glucoflex-R Visual Blood Glucose Strips } \\
\hline & Solution 1 & Solution 2 & Solution 3 & $\begin{array}{l}\text { Hyclone High Glucose } \\
450 \text { mg/dL Calibration }\end{array}$ \\
\hline $\begin{array}{c}\text { Average } \\
\text { Glucose } \\
\text { Concentration } \\
(\mathbf{m g} / \mathbf{d L})\end{array}$ & 205.00 & 194.00 & 312.00 & N/A \\
\hline $\begin{array}{l}\text { Standard } \\
\text { Deviation }\end{array}$ & 53.59 & 109.16 & 199.38 & N/A \\
\hline$\%$ Error & $2.50 \%$ & $-3.00 \%$ & $56.00 \%$ & N/A \\
\hline
\end{tabular}

Lastly, a two-tailed T-test was conducted to compare the mean glucose concentrations of each solution based on the method of measurement. The null hypothesis was that the mean glucose concentration accumulated by the Aviva Accu-Chek blood glucose meter was equal to the mean glucose concentration accumulated by the Glucoflex-R visual test strips $\left(\mathrm{H}_{\mathrm{o}}: \chi_{\text {Accu-chek }}=\right.$ $\left.\chi_{\text {Glucoflex-R }}\right)$. Thus, the alternative hypothesis was that these two means were not equal $\left(\mathrm{H}_{\mathrm{a}}: \chi_{\text {Accu- }}\right.$ Chek $\left.\neq \chi_{\text {Glucoflex-R }}\right)$. With a 95\% confidence interval $(\alpha=0.05)$, all $p$-values were greater than 0.05 ; 
thus, not rejecting the null hypothesis, and confirming that there was no statistical significant difference.

In addition, an ANOVA test was conducted to compare the mean glucose concentrations of all three solutions within each method of measurement. The null hypotheses were that the mean glucose concentration of each solution measured by the Aviva Accu-Chek blood glucose meter were equal $\left(\mathrm{H}_{\mathrm{o}}: \chi_{\text {Solution } 1}=\chi_{\text {Solution } 2}=\chi_{\text {Solution } 3}\right)$, and that the mean glucose concentration of each solution measured by the Glucoflex-R visual test strips were equal as well $\left(\mathrm{H}_{\mathrm{o}}: \chi_{\text {Solution1 }}=\right.$

$\left.\chi_{\text {Solution2 }}=\chi_{\text {Solution } 3}\right)$. Once again, a 95\% confidence interval was used $(\alpha=0.05)$, and the resulting $p$-values were greater than 0.05 ; thus, confirming no statistical significant difference among the average glucose concentrations of each solution within the specific method of measurement. Both the two-tailed T-tests and ANOVA tests indicated that the accumulated glucose concentrations were statistically similar, and recording a concentration consistently close to the expected concentration of $200 \mathrm{mg} / \mathrm{dl}$. However, the high variance of the Glucoflex-R visual strips was not an ideal attribute for the purposes of further experimentation.

\subsubsection{Hyperglycemic Solution (350 $\mathrm{mg} / \mathrm{dL})$}

A hyperglycemic solution of $350 \mathrm{mg} / \mathrm{dL}$ was created to replicate a hyperglycemic environment. The established protocol called for $0.250 \mathrm{~g}$ of glucose to be weighed and added to $100 \mathrm{~mL}$ of media for each solution. Table 5 displays the exact amount of glucose added to each volume of cell media.

Table 5 - Amount of Sigma G6152 D-(+)-Glucose added to $100 \mathrm{~mL}$ of Gibco Medium 199 for the $350 \mathrm{mg} / \mathrm{dL}$ hyperglycemic solutions

\section{\begin{tabular}{|l|l|l|}
\hline Solution Sample & Amount of Glucose Added & Volume of Cell Media (mL) \\
\hline
\end{tabular}}




\begin{tabular}{|c|c|c|}
\hline & $(\mathrm{g})$ & 100 \\
\hline $\mathbf{1}$ & 0.2506 & 100 \\
\hline $\mathbf{2}$ & 0.2501 & 100 \\
\hline
\end{tabular}

As with the $200 \mathrm{mg} / \mathrm{dL}$ solutions, after recording glucose concentrations for $\mathrm{t}=48$ hours, the accumulated results matched closely with the expected concentration of $350 \mathrm{mg} / \mathrm{dL}$.

Figure 32 shows that the recorded glucose concentrations for each solution, using the Aviva Accu-Chek blood glucose meter, were very close to expected concentration of $350 \mathrm{mg} / \mathrm{dL}$. While it took approximately two hours for the $200 \mathrm{mg} / \mathrm{dL}$ solutions to settle at a consistent concentration, the concentrations of the $350 \mathrm{mg} / \mathrm{dL}$ solutions gradually increased for the first few hours and finally settled after approximately four hours of mixing.

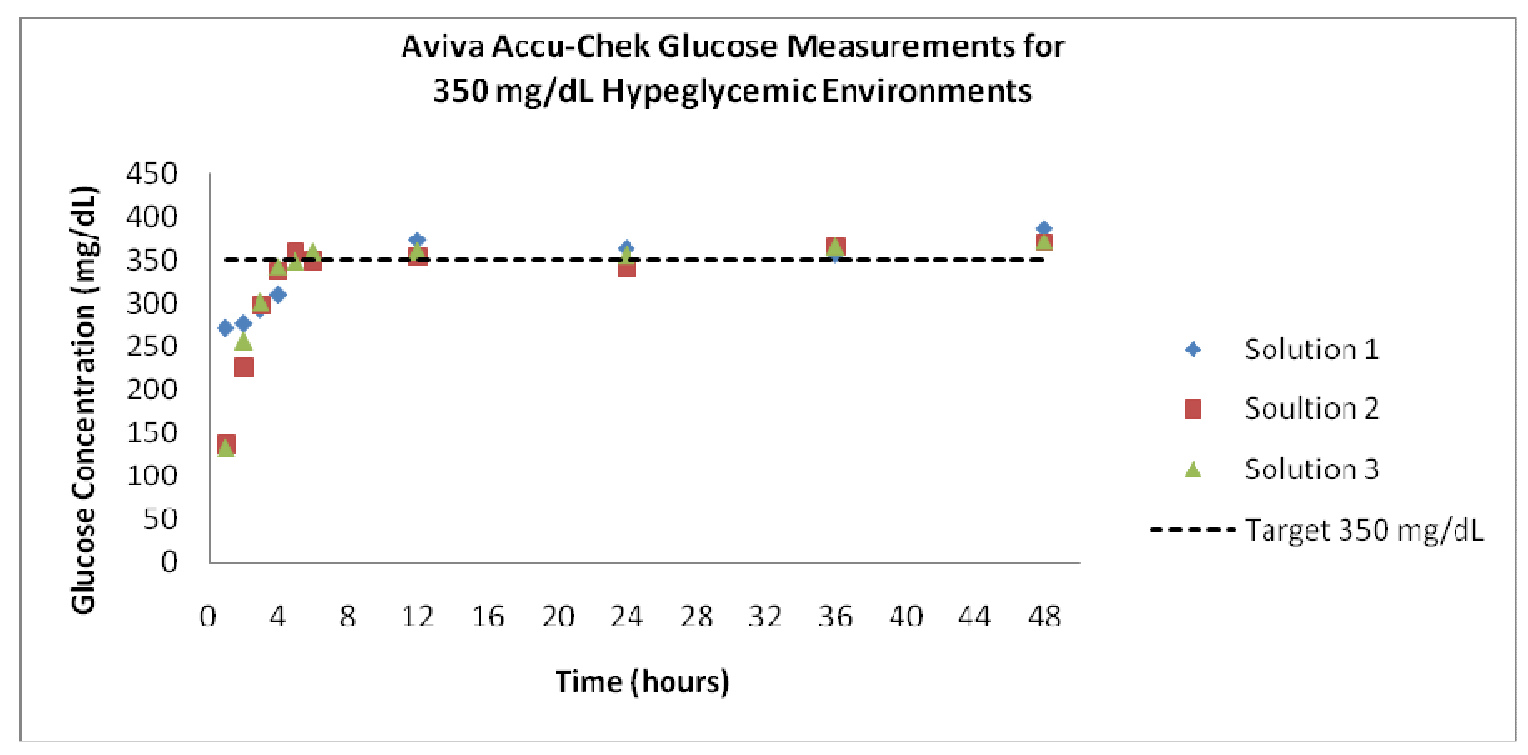

Figure 32 - The plot shows that the glucose concentrations recorded by the Aviva Accu-Chek blood glucose meter were similar to the expected glucose concentration of $350 \mathrm{mg} / \mathrm{dL}$ for each solution. The glucose concentration rose rapidly, but settled after approximately four hours of mixing.

As for the Glucoflex-R visual test strips, Figure 33 shows results similar to that of the $200 \mathrm{mg} / \mathrm{dL}$ solutions. The visual test strips were able to consistently record a glucose 
concentration, throughout the 72 hour experimentation time, that was within range of the expected $350 \mathrm{mg} / \mathrm{dL}$ concentration. However, the reoccurrence of high variance of the recorded glucose measurements continued to question the precision of the visual test strips. Recall, a high variance is not an ideal characteristic for a method of measurement glucose concentrations considering the sensitivity of any glucose environments.

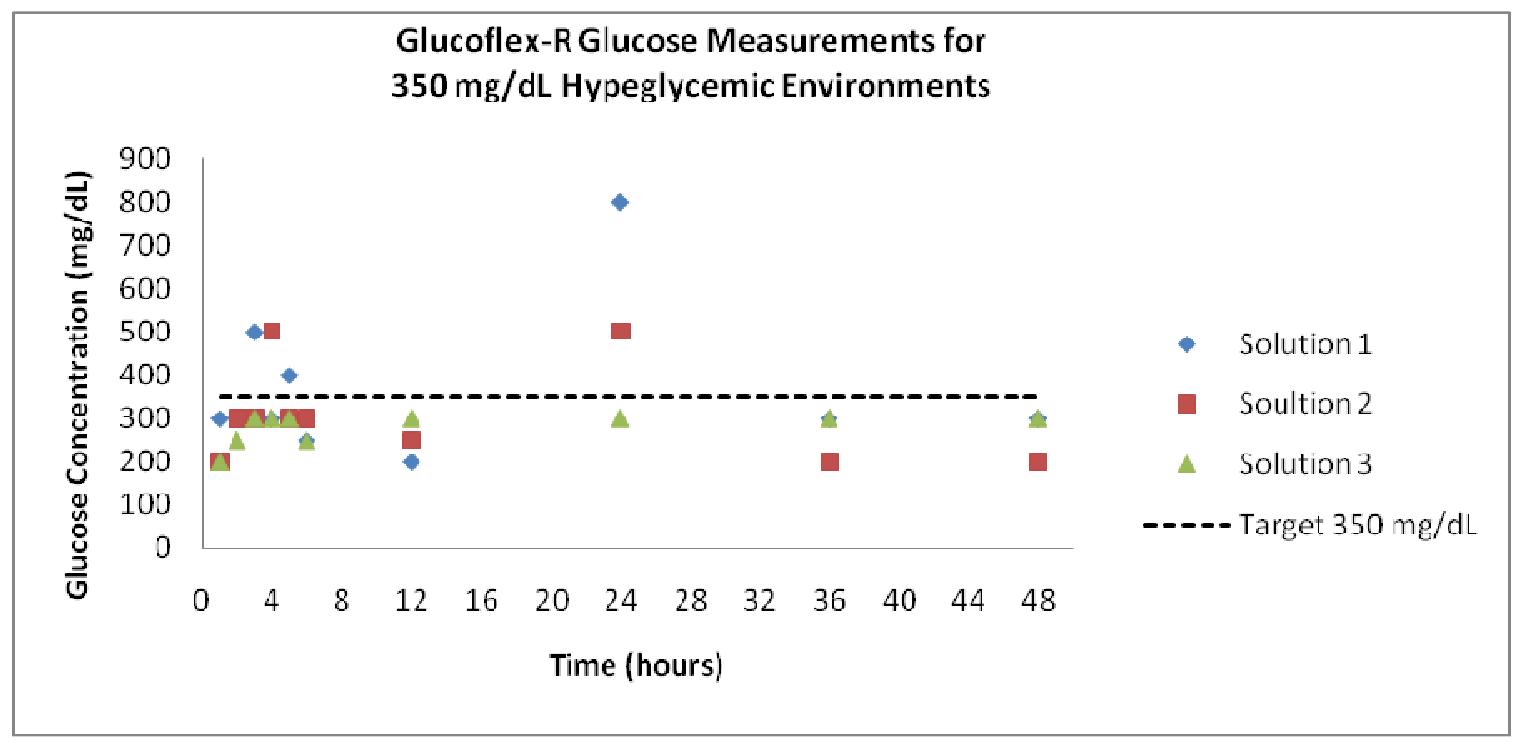

Figure 33 - The recorded glucose concentrations, using the Glucoflex-R visual test strips, for the $350 \mathrm{mg} / \mathrm{dL}$ solutions, were within range of the expected glucose concentration. However, the extremely high variance further proved the lack of precision of the visual test strips.

Table 6 shows the average glucose concentrations for each solution recorded by the Aviva Accu-Chek blood glucose meter and the Glucoflex-R visual test strips, along with the standard deviation and percent error from the expected glucose concentration of $350 \mathrm{mg} / \mathrm{dL}$. Since it took approximately four hours for the solutions to mix uniformly, the average glucose concentration for each solution was calculated from $t=4$ hours to $t=48$ hours. This limited the amount of variance in the data since the glucose concentrations recorded from $t=1$ to $t=3$ hours did not properly represent the actual glucose concentration of each solution. Looking at Error! Reference source not found.Table 6, the Aviva Accu-Chek blood glucose meter recorded 
concentrations close to the expected concentration of $350 \mathrm{mg} / \mathrm{dL}$ with limited variance. The glucose concentrations recorded by the Glucoflex-R visual test strips, however, once again had extremely high variance and were not as precise as the Aviva Accu-Chek blood glucose meter.

Table 6 - Average Glucose Concentrations Recorded with Accu-Chek Blood Glucose Meter \& Glucoflex-R Visual Blood Glucose Strips for 350 mg/dL Hyperglycemic Solutions

\begin{tabular}{|c|c|c|c|c|}
\hline \multicolumn{5}{|c|}{ A viva Accu-Chek Blood Glucose Meter } \\
\hline & Solution 1 & Solution 2 & Solution 3 & $\begin{array}{l}\text { Hyclone High Glucose } \\
450 \mathrm{mg} / \mathrm{dL} \text { Calibration }\end{array}$ \\
\hline $\begin{array}{c}\text { Average } \\
\text { Glucose } \\
\text { Concentration } \\
(\mathbf{m g} / \mathbf{d L}) \\
(\mathrm{t}=\mathbf{4})\end{array}$ & 356.71 & 354.00 & 357.57 & 449.00 \\
\hline $\begin{array}{c}\text { Standard } \\
\text { Deviation } \\
(\text { from } \mathbf{t}=\mathbf{4}) \\
\end{array}$ & 23.91 & 11.54 & 9.91 & 13.06 \\
\hline$\%$ Error & $1.92 \%$ & $1.14 \%$ & $2.16 \%$ & $-0.222 \%$ \\
\hline \multicolumn{5}{|c|}{ Glucoflex-R Visual Blood Glucose Strips } \\
\hline & Solution 1 & Solution 2 & Solution 3 & $\begin{array}{l}\text { Hyclone High Glucose } \\
450 \mathrm{mg} / \mathrm{dL} \text { Calibration }\end{array}$ \\
\hline $\begin{array}{c}\text { Average } \\
\text { Glucose } \\
\text { Concentration } \\
(\mathbf{m g} / \mathbf{d L}) \\
(\mathbf{t}=4)\end{array}$ & 364.29 & 321.43 & 292.86 & N/A \\
\hline $\begin{array}{c}\text { Standard } \\
\text { Deviation } \\
(t=4) \\
\end{array}$ & 201.48 & 128.64 & 18.90 & N/A \\
\hline$\%$ Error & $4.08 \%$ & $-8.16 \%$ & $-16.33 \%$ & N/A \\
\hline
\end{tabular}

Again, to finalize the analysis, a two-tailed T-test was conducted to compare the mean glucose concentrations of each solution based on the method of measurement. The null hypothesis was that the mean glucose concentration accumulated by the Aviva Accu-Chek blood 
glucose meter was equal to the mean glucose concentration accumulated by the Glucoflex-R visual test strips $\left(\mathrm{H}_{\mathrm{o}}=\chi_{\text {Accu-Chek }}=\chi_{\text {Glucoflex-R }}\right)$. Thus, the alternative hypothesis was that these two means were not equal $\left(\mathrm{H}_{\mathrm{a}}=\chi_{\text {Accu-Chek }} \neq \chi_{\text {Glucoflex-R }}\right)$. With a $95 \%$ confidence interval $(\alpha=0.05)$, all $p$-values were greater than 0.05 , therefore, not rejecting the null hypothesis and confirming that there was no statistical significant difference between the means of the Aviva Accu-Chek blood glucose meter and the Glucoflex-R visual test strips.

Lastly, an ANOVA test was conducted to compare the mean glucose concentrations of each solution within each method of measurement. The null hypotheses were that the mean glucose concentration of each solution measured by the Aviva Accu-Chek meter were equal $\left(\mathrm{H}_{\mathrm{o}}\right.$ $\left.=\chi_{\text {Solution } 1}=\chi_{\text {Solution } 2}=\chi_{\text {Solution } 3}\right)$, and that the mean glucose concentration of each solution measured by the Glucoflex-R visual test strips were equal as well $\left(\mathrm{H}_{\mathrm{o}}=\chi_{\text {Solution } 1}=\chi_{\text {Solution2 }}=\right.$ $\left.\chi_{\text {Solution3 }}\right)$. Once again, a 95\% confidence interval was used $(\alpha=0.05)$, and both resulting $p$ values were greater than 0.05 , therefore, not rejecting the null hypothesis and confirming that there was no statistical significant difference between the means of each solution within the method of measurement.

Although statistical analysis confirmed that the glucose concentrations recorded by the Glucoflex-R visual test strips were not statistically different from the glucose concentrations recorded by the Aviva Accu-Chek blood glucose meter, the variance of the data accumulated with the Glucoflex-R visual test strips was even higher than the variance from the $200 \mathrm{mg} / \mathrm{dL}$ solutions. It was possible that the accuracy of the Glucoflex-R visual test strips decreased as the glucose concentrations of the tested solutions increased. 


\subsubsection{Extreme Hyperglycemic Solution (500 $\mathrm{mg} / \mathrm{dL})$}

A hyperglycemic solution of $500 \mathrm{mg} / \mathrm{dL}$ was created to replicate an extremely hyperglycemic environment. The established protocol called for $0.400 \mathrm{~g}$ of glucose to be weighed and added to $100 \mathrm{~mL}$ of media for each solution. Table 7 displays the exact amounts of glucose added to each volume of cell media.

Table 7 - Amount of Sigma G6152 D-(+)-Glucose added to $100 \mathrm{~mL}$ of Gibco Medium 199 for $500 \mathrm{mg} / \mathrm{dL}$ extreme hyperglycemic solutions

\begin{tabular}{|c|c|c|}
\hline Solution Sample & $\begin{array}{c}\text { Amount of Glucose Added } \\
(\mathrm{g})\end{array}$ & Volume of Cell Media (mL) \\
\hline 1 & 0.4006 & 100 \\
\hline 2 & 0.4002 & 100 \\
\hline 3 & 0.4003 & 100 \\
\hline
\end{tabular}

After accumulating the data, the results were very similar to those of the $200 \mathrm{mg} / \mathrm{dL}$ and $350 \mathrm{mg} / \mathrm{dL}$ solutions.

Figure 34 shows the plot of measured glucose concentrations for each $500 \mathrm{mg} / \mathrm{dL}$ solution using the Aviva Accu-Chek blood glucose meter. The plot shows that the measured glucose concentrations were very close to the expected concentration of $500 \mathrm{mg} / \mathrm{dL}$. In addition, the plot shows that it took approximately five hours for the solutions to mix uniformly. This was not a surprise since it took approximately four hours for the $350 \mathrm{mg} / \mathrm{dL}$ solutions to mix completely. 


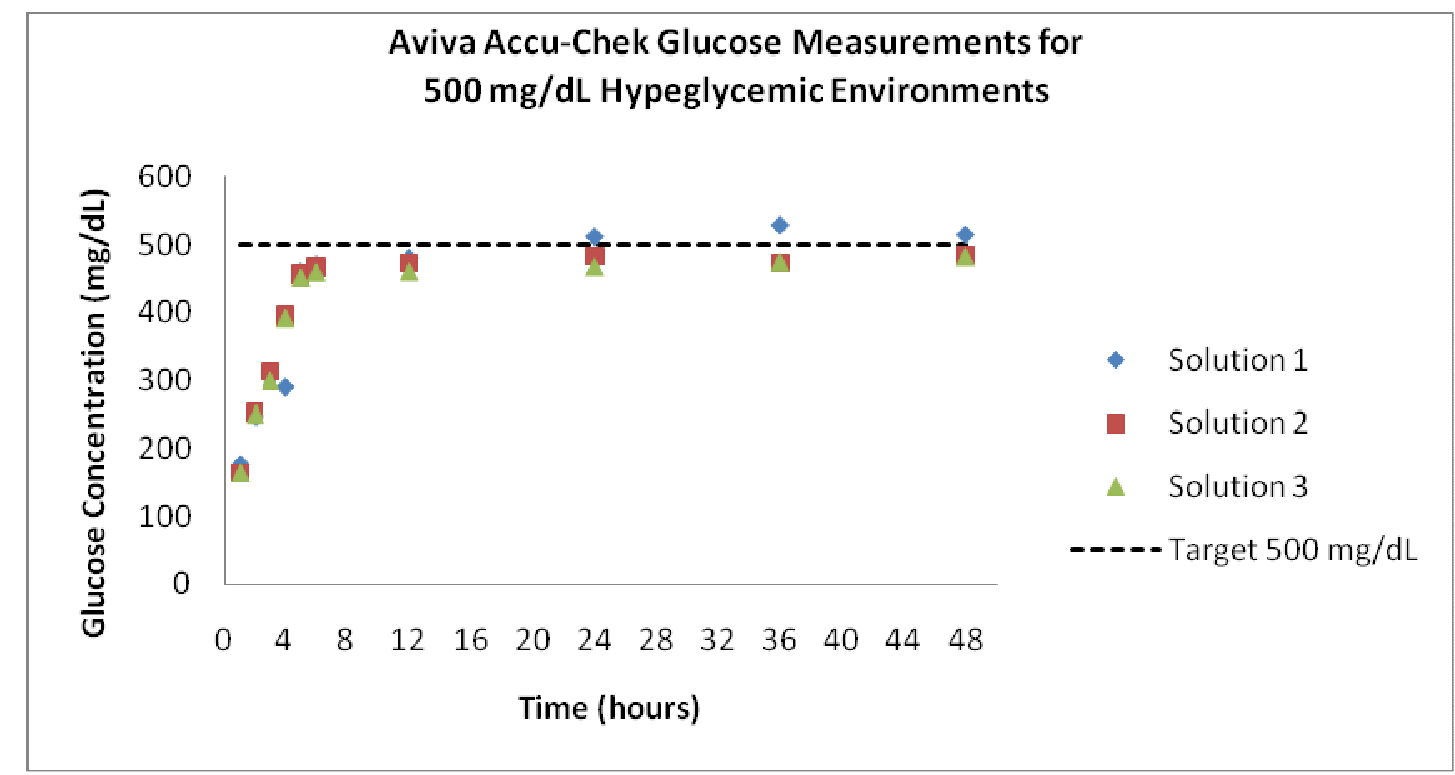

Figure 34 - Even though the Accu-Chek glucose meter continued to measure glucose concentrations higher than the expected value of $400 \mathrm{mg} / \mathrm{dL}$, the average measurements were much more accurate compared to the average measurements for the other glycemic environments.

Figure 35 shows a plot of measured glucose concentrations for each $500 \mathrm{mg} / \mathrm{dL}$ solution using the Glucoflex-R visual test strips. As with the results from the $200 \mathrm{mg} / \mathrm{dL}$ and $350 \mathrm{mg} / \mathrm{dL}$ solutions, the visual test strips were able to consistently measure a glucose concentration for each solution throughout the test, but the variation of the measurements was again very high relative to the variation of the Aviva Accu-Chek blood glucose meter. 


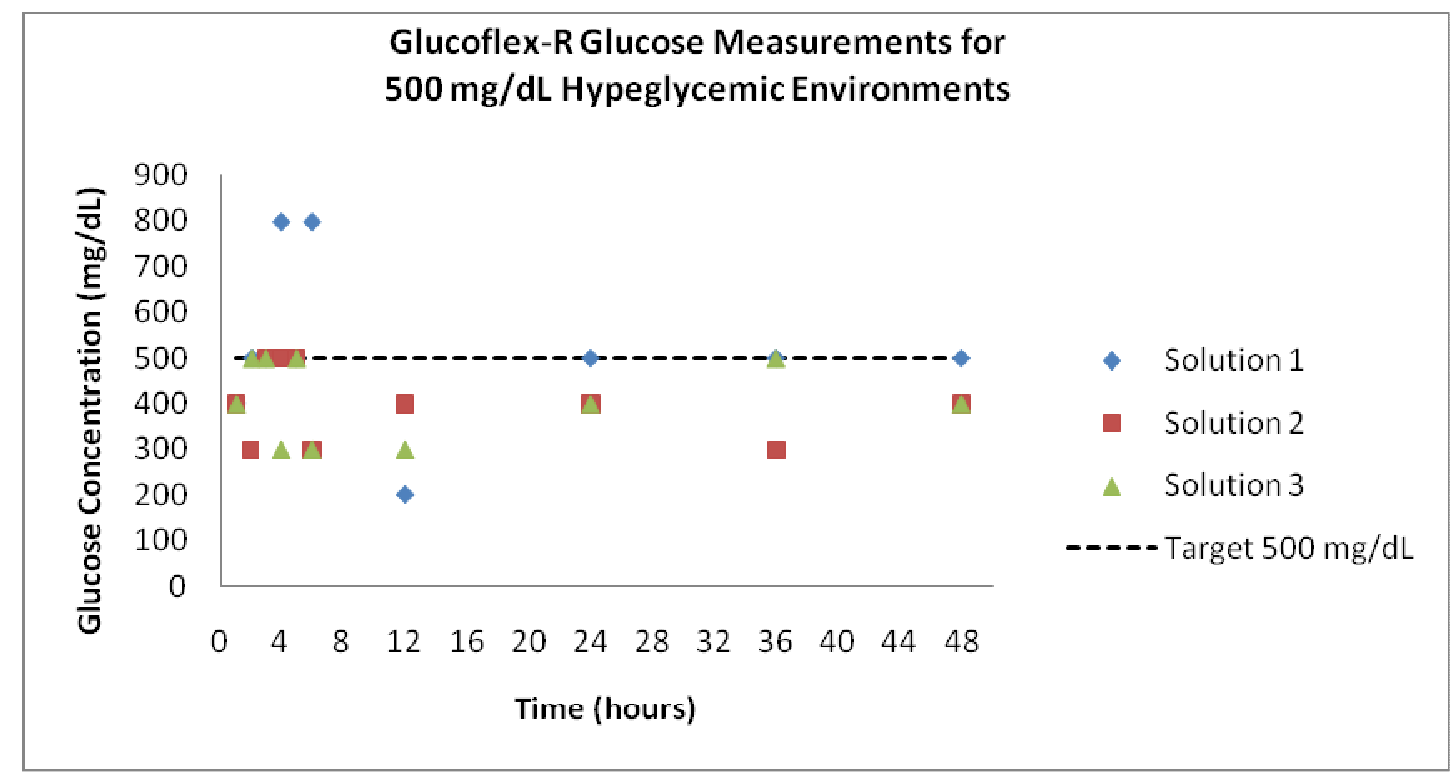

Figure 35 - The Glucoflex-R strips were able to monitor the glucose concentrations of the extremely hyperglycemic $400 \mathrm{mg} / \mathrm{dL}$ solutions much more accurately than the other glycemic environments.

Error! Reference source not found.Table 8 shows that the calculated average glucose concentrations obtained from the Aviva Accu-Chek blood glucose meter and the Glucoflex-R visual test strips. Recall, it took approximately five hours for the solution to mix uniformly; thus, the averages were taken from $t=5$ hours to $t=48$ hours. The calculated averages from the Aviva Accu-Chek blood glucose meter were extremely close to the targeted value of $500 \mathrm{mg} / \mathrm{dL}$, resulting in a very low percent error for each solution. In addition, the Aviva Accu-Chek blood glucose meter was able to measure glucose concentrations with limited variance (higher variance than $200 \mathrm{mg} / \mathrm{dL}$ and $350 \mathrm{mg} / \mathrm{dL}$ solutions, but expected with the increased amount of glucose added to the solutions). While the calculated average glucose concentrations obtained from the Glucoflex-R visual test strips were close to the expected glucose concentration as well (solution 1 even hit $500 \mathrm{mg} / \mathrm{dL}$ exactly), the significant factor once again was the relatively high variance of the Glucoflex-R data, especially in relation to the low variance seen with the data accumulated by the Aviva Accu-Chek blood glucose meter. This confirms that, while the visual test strips are 
able to consistently measure the glucose concentrations throughout the test, the precision of the blood glucose meter is much higher than that of the visual test strips in all three tested levels of glucose concentration.

Table 8 - Average Glucose Concentrations Recorded with Accu-Chek Blood Glucose Meter \& Glucoflex-R Visual Blood Glucose Strips for $500 \mathrm{mg} / \mathrm{dL}$ extreme hyperglycemic solutions

\begin{tabular}{|c|c|c|c|c|}
\hline \multicolumn{5}{|c|}{ Aviva Accu-Chek Blood Glucose Meter } \\
\hline & Solution 1 & Solution 2 & Solution 3 & $\begin{array}{l}\text { Hyclone High Glucose } \\
450 \text { mg/dL Calibration }\end{array}$ \\
\hline $\begin{array}{c}\text { Average } \\
\text { Glucose } \\
\text { Concentration } \\
(\mathbf{m g} / \mathbf{d L}) \\
(\mathbf{t}=\mathbf{5})\end{array}$ & 493.33 & 472.67 & 466.50 & 447.33 \\
\hline $\begin{array}{l}\text { Standard } \\
\text { Deviation } \\
(\text { from } \mathbf{t}=\mathbf{5})\end{array}$ & 27.990 & 10.482 & 11.221 & 6.250 \\
\hline$\%$ Error & -1.33 & -5.48 & -6.70 & N/A \\
\hline \multicolumn{5}{|c|}{ Glucoflex-R Visual Blood Glucose Strips } \\
\hline & Solution 1 & Solution 2 & Solution 3 & $\begin{array}{l}\text { Hyclone High Glucose } \\
450 \text { mg/dL Calibration }\end{array}$ \\
\hline $\begin{array}{c}\text { Average } \\
\text { Glucose } \\
\text { Concentration } \\
(\mathbf{m g} / \mathbf{d L}) \\
(\mathbf{t}=\mathbf{5})\end{array}$ & 500.00 & 383.33 & 400.00 & N/A \\
\hline $\begin{array}{l}\text { Standard } \\
\text { Deviation } \\
(t=5)\end{array}$ & 189.737 & 75.277 & 89.443 & N/A \\
\hline$\%$ Error & 0.00 & -23.33 & -20.00 & N/A \\
\hline
\end{tabular}


Lastly, a two-tailed t-Test was conducted to compare the mean glucose concentrations of each solution based on the method of measurement. The null hypothesis was that the mean glucose concentration accumulated by the Aviva Accu-Chek blood glucose meter was equal to the mean glucose concentration accumulated by the Glucoflex-R visual test strips $\left(\mathrm{H}_{\mathrm{o}}=\chi_{\text {Accu-Chek }}\right.$ $\left.=\chi_{G \text { Glucoflex-R }}\right)$. Thus, the alternative hypothesis was that these two means were not equal $\left(\mathrm{H}_{\mathrm{a}}=\right.$ $\left.\chi_{\text {Accu-Chek }} \neq \chi_{\text {Glucoflex-R }}\right)$. With a 95\% confidence interval $(\alpha=0.05)$, all $p$-values were greater than 0.05 , therefore, not rejecting the null hypothesis and confirming that there was no statistical significant difference between the means of the Aviva Accu-Chek blood glucose meter and the Glucoflex-R visual test strips.

Lastly, an ANOVA test was conducted to compare the mean glucose concentrations of each solution within each method of measurement. The null hypotheses were that the mean glucose concentration of each solution measured by the Aviva Accu-Chek meter were equal $\left(\mathrm{H}_{\mathrm{o}}\right.$ $=\chi_{\text {Solution } 1}=\chi_{\text {Solution } 2}=\chi_{\text {Solution3 } 3}$, and that the mean glucose concentration of each solution measured by the Glucoflex-R visual test strips were equal as well $\left(\mathrm{H}_{\mathrm{o}}=\chi_{\text {Solution } 1}=\chi_{\text {Solution } 2}=\right.$ $\left.\chi_{\text {Solution3 } 3}\right)$. Once again, a $95 \%$ confidence interval was used $(\alpha=0.05)$, and both resulting $p$ values were greater than 0.05 (though, the $p$-value for the ANOVA test conducted on the Glucoflex-R solutions was very close to 0.05 , further confirming the troubles of high variance), therefore, not rejecting the null hypothesis and confirming that there was no statistical significant difference between the means of each solution within the method of measurement.

\subsection{Discussion and Conclusions}

Recall, the goals of this chapter were the following: 
1. To explore and identify practical methods of measuring levels of glucose in different glycemic cell media solutions;

2. To develop and establish a procedure to create hyperglycemic cell media solutions with the addition of glucose into base cell media; and

3. To use the hyperglycemic cell media solutions in order to evaluate the different methods of monitoring glucose concentrations and determine the most feasible method.

Reviewing the literature confirmed that the most practical methods of monitoring glucose concentrations of cell media solutions were the blood glucose meter and the visual blood glucose test strip. Additionally, preliminary tests showed that the Aviva Accu-Chek glucose meter and Glucoflex-R visual blood glucose strips gave accurate and reliable measurements. Therefore, the Aviva Accu-Chek blood glucose meter and the Glucoflex-R visual blood glucose test strips were used to measure glucose concentrations of three glycemic cell media solutions $200 \mathrm{mg} / \mathrm{dL}, 350 \mathrm{mg} / \mathrm{dL}$, and $500 \mathrm{mg} / \mathrm{dL}$.

The results from the experiments in this chapter indicated that a high glucose concentrated solution, with Sigma G6152 D-(+)-Glucose and Gibco Medium 199, can properly represent a hyperglycemic environment. In addition, the Aviva Accu-Chek blood glucose meter, over a 48 hour time period, was able to accurately and precisely monitor and record glucose concentrations very close to the expected concentration of each glycemic cell media solution. Though the Glucoflex-R visual test strips were able to consistently record an average glucose concentration close to the expected concentration, the variability of the visual test strips was very high - a characteristic that would be extremely detrimental to the data acquisition of further experiments. In addition, although t-tests confirmed that there was not a statistical significant 
difference between the averages accumulated by the Aviva Accu-Chek blood glucose meter and averages accumulated with the Glucoflex-R visual test strips, the combination of a low percent error with a low standard deviation made the Aviva Accu-Chek blood glucose meter the better option for evaluation of glucose concentration for glycemic cell media solutions.

One key observation was the amount of time necessary to ensure that all the added glucose completely mixed into the cell media. While the $200 \mathrm{mg} / \mathrm{dL}$ media took relatively little time to uniformly mix, the $350 \mathrm{mg} / \mathrm{dL}$ and $500 \mathrm{mg} / \mathrm{dL}$ solutions needed approximately four to five hours of mixing before the solution mixed to its expected concentration. This time was taken into consideration when establishing the protocol for developing glycemic cell media solutions (Appendix D).

The greatest challenge of the experiments in this chapter was adding an accurate amount of Sigma G6152 D-(+)-Glucose to the $100 \mathrm{~mL}$ of cell media. Even in a large volume of media, an extremely small amount of glucose can cause a great fluctuation in the glucose concentration. This error was limited by ensuring that a very small amount of additional glucose was weighed to compensate for any lost glucose during transfer from the weigh boat to the solution. The calculated percent errors of the results confirmed that this test procedure was executed correctly. A possible way to avoid this complication, however, would be to use an even greater volume of cell media. Adding glucose to $100 \mathrm{~mL}$ of cell media to achieve $200 \mathrm{mg} / \mathrm{dL}$ leaves an extremely small margin of error; thus, it would be beneficial to increase the cell media volume to $500 \mathrm{~mL}$ or even $1000 \mathrm{~mL}$ if necessary.

In addition, the concentrations that were used were slightly higher than the initial target values, due to the existing glucose in M199. If cell media with $1000 \mathrm{mg} / \mathrm{L}(100 \mathrm{mg} / \mathrm{dL})$ of D- 
glucose is used in the future, here are the recipes that should be used for achieving $100 \mathrm{mg} / \mathrm{dl}$, 250mg/dl, and 400mg/dl as initially intended:

- $100 \mathrm{mg} / \mathrm{dL}$

○ $100 \mathrm{~mL}$ of M199 only

*Gibco Medium 199 already contains 1000 mg/L (100 mg/dL) of Dglucose; thus, no additional modifications are necessary

- $250 \mathrm{mg} / \mathrm{dL}$

○ Add 0.15 g of Sigma G6152 D-(+)-Glucose to $100 \mathrm{~mL}$ of M199

- $400 \mathrm{mg} / \mathrm{dL}$

○ Add $0.30 \mathrm{~g}$ of Sigma G6152 D-(+)-Glucose to $100 \mathrm{~mL}$ of M199

One last note was that the test initially called for two blood glucose meters to be used to measure the glucose concentrations of the glycemic cell media solutions (the Bayer Contour blood glucose meter along with the Aviva Accu-Chek blood glucose meter). Unfortunately, the Bayer blood glucose meter was unable to give any recording at all as it consistently gave an "error" message. One reason for this was probably due to the meter's inability to analyze glucose when it is in its cyclic structure. Recall, that at a neutral $\mathrm{pH}$ of $7.0, \mathrm{D}$-glucose is in its cyclic form. The human body is not always at a $\mathrm{pH}$ of 7.0 ; thus, it is possible that glucose circulating in blood is not in cyclic form and glucose meters accurately measure glucose concentrations of blood only when it is in its straight chain form. The Gibco Medium 199 has a $\mathrm{pH}$ of about 7.4 meaning that the tested glycemic solutions probably contained glucose in its cyclic form.

The series of experiments in this chapter both established a procedure of creating glycemic cell media solutions with the addition of Sigma G6152 D-(+)-Glucose to Gibco 
Medium 199, as well as a method of monitoring the glucose concentration of these solutions with the Aviva Accu-Chek blood glucose meter. The next step of this thesis study involved the incorporation of the developed glycemic solutions with endothelial cells, in an attempt to determine whether or not the glycemic cell media solutions evoked a negative response by endothelial cells. This work will be described in the next chapter. 


\section{Chapter 3: Evaluation of Endothelial Cells in High}

\section{Glucose Solutions}

\subsection{Introduction}

In Chapter 2, hyperglycemia - the major dysfunction of diabetes mellitus - was discussed. It was determined that hyperglycemia was the most practical attribute to take into consideration when trying to replicate a diabetic environment. Thus, in order to create and monitor a hyperglycemic environment, a protocol was established to develop high glucose concentrated solutions using Sigma G6152 D-(+)-Glucose and Gibco Medium 199, and different methods of measuring glucose concentrations were discussed. After reviewing the literature, the Aviva Accu-Chek blood glucose meter and the Glucoflex-R visual blood glucose test strips were the most feasible methods of measuring the glucose concentration of various glycemic cell media solutions. Three different glycemic cell media solutions were developed to resemble three glycemic environments:

1. Normal glycemic level: $100 \mathrm{mg} / \mathrm{dL}$

2. Hyperglycemic level: $250 \mathrm{mg} / \mathrm{dL}$

3. Extreme Hyperglycemic level: $400 \mathrm{mg} / \mathrm{dL}$

It was determined that the Aviva Accu-Chek blood glucose meter was the best method to measure the glucose concentrations of glycemic cell media solutions because of its ability to record very accurate and precise result,. In addition, it was uncovered that the Gibco Medium 199 was a "low glucose" solution and already contained $1000 \mathrm{mg} / \mathrm{L}(100 \mathrm{mg} / \mathrm{dL})$ of glucose. 
Therefore, it was necessary to compensate and adjust the amount of glucose added to the cell media when creating the glycemic cell media solutions for further experiments.

The next step then was the incorporation of the developed glycemic cell media solutions with endothelial cells in the attempt to determine whether or not the hyperglycemic cell media solutions evoked a noticeable negative response in endothelial cells. Specifically, bovine aortic endothelial cells (BAECs) and human umbilical vein endothelial cells (HUVECs) were used to evaluate the effect of hyperglycemic cell media recipes. The BAECs and HUVECs in this study were exposed to two different glycemic environments - a normal glycemic environment of 100 $\mathrm{mg} / \mathrm{dL}$, and a hyperglycemic environment of $400 \mathrm{mg} / \mathrm{dL}$. The development of these recipes will be discussed in the proceeding "Methods" section.

Remember, a very small amount of glucose is circulating in the body (roughly 5 grams of glucose in the average human body). Thus, the chance of error due to a minor miscalculation in the amount of glucose added to the cell media is relatively high. Therefore, the hyperglycemic environment of $350 \mathrm{mg} / \mathrm{dL}$, which was tested in the previous chapter, was removed from experiments in this chapter to avoid any overlapping. Therefore, experimentation with BAECs and HUVECs focused entirely on a $100 \mathrm{mg} / \mathrm{dL}$ normal glycemic environment, and a $400 \mathrm{mg} / \mathrm{dL}$ hyperglycemic environment.

\subsubsection{Bovine Aortic Endothelial Cells (BAECs)}

Bovine aortic endothelial cells are vascular cells from the aortic valve of USDA-cattle. These cells are usually preserved at second passage and can be cultured and passed for at least sixteen population doublings [139]. These cells play a critical role in cardiac homeostasis; thus, studies have linked changes in structure and function of these endothelial cells to vascular 
diseases such as thrombosis, atherosclerosis, and hypertension - all significant complications of diabetes mellitus [139]. Therefore, these endothelial cells are a suitable and economical option to study endothelial function and metabolism.

Researchers have used BAECs to study the effects of elevated glucose levels on cultured endothelial cells [140]. One study showed that hyperglycemia inhibits endothelial nitric oxide activity in BAECs; nitric oxide synthase 3 (eNOS) plays an important role in preventing vascular diseases [141]. Figure 36 shows how increased glucose concentration hindered eNOS activity. BAECs exposed to a $5 \mathrm{mM}$ glucose environment $(90 \mathrm{mg} / \mathrm{dL})$ had much higher eNOS activity than BAECs exposed to a $30 \mathrm{mM}$ environment $(540 \mathrm{mg} / \mathrm{dL})$.
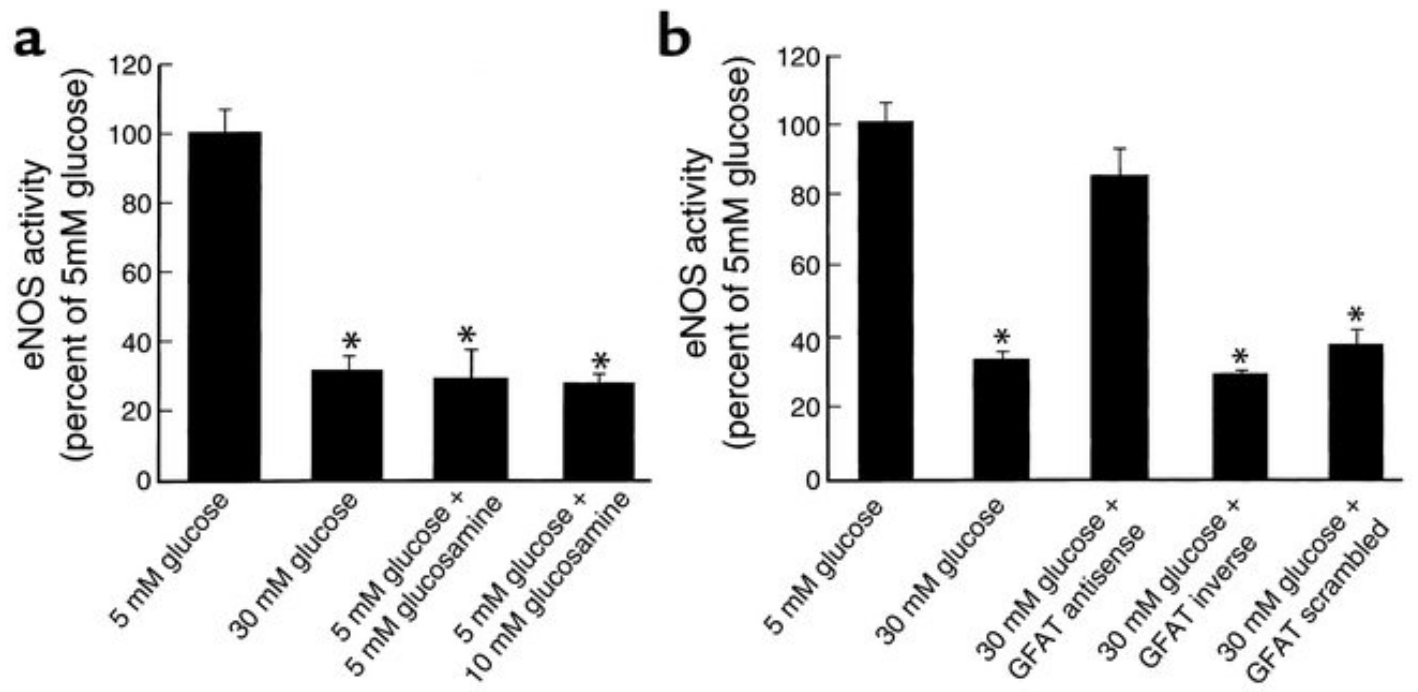

Figure 36 - This study showed how exposure of BAECs to a hyperglycemic environment hindered nitric oxide (eNOS) activity. BAECs exposed to a $5 \mathrm{mM}(90 \mathrm{mg} / \mathrm{dL})$ normal glycemic environment had higher eNOS activity than BAECs exposed to the $30 \mathrm{mM}(540 \mathrm{mg} / \mathrm{dL})$ hyperglycemic environment. eNOS plays an important role in preventing many vascular diseases (permission to reprint from [141]).

Another study showed that hyperglycemia increased intracellular reactive oxygen species (ROS) production in cultured BAECs [142]. The study showed that ROS activates mechanisms that link hyperglycemia to several functional pathways that are responsible for the microvascular 
damage induced by diabetes [142]. These mechanisms include an altered cellular redox state, an increase in diacylglycerol (DAG), which in turn adds the subsequent activation of protein kinase $\mathrm{C}(\mathrm{PKC})$ isoforms [143]. Recall from the beginning of Chapter 2 that the activation of the DAGPKC pathway has been associated with many vascular abnormalities in the retinal, renal, neural and cardiovascular tissues in diabetes mellitus. The DAG-PKC pathway affects the regulation of endothelial cell permeability, vasoconstriction, extracellular matrix (ECM) synthesis, endothelial cell growth, and angiogenesis [143]. These studies are a few of the examples that support the use of BAECs in a hyperglycemic environment as a suitable method to study the function and metabolism of endothelial cells in vitro.

\subsubsection{Human Umbilical Vein Endothelial Cells (HUVECs)}

Human umbilical vein endothelial cells (HUVECs) are isolated from human umbilical veins. These cells are responsive to cytokine stimulation in the expression of cell adhesion molecules; thus, these cells are primarily used to study physiological and pharmacological functions, such as macromolecule transport, blood coagulation, and fibrinolysis [144].

A previous study had shown that incubation of HUVECs in high glucose environments (roughly $30 \mathrm{mmol} / \mathrm{L}$ or $540 \mathrm{mg} / \mathrm{dL}$ ) for 72 hours caused a significant increase in apoptosis, possibly related to increased oxidative stress $[145,146]$. The study showed that the hyperglycemic environment altered fatty-acid metabolism, which in turn hindered Akt activation by insulin (Akt phosphorylation usually occurs in the presence of insulin that induces optimum metabolism of glucose). In addition, there was an increase in caspase- 3 activity preceding HUVEC apoptosis in the hyperglycemic environment (Figure 37). 
A

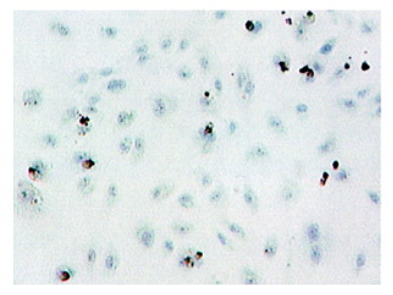

$5 \mathrm{mmol} / \mathrm{l} \mathrm{glucose}$

B

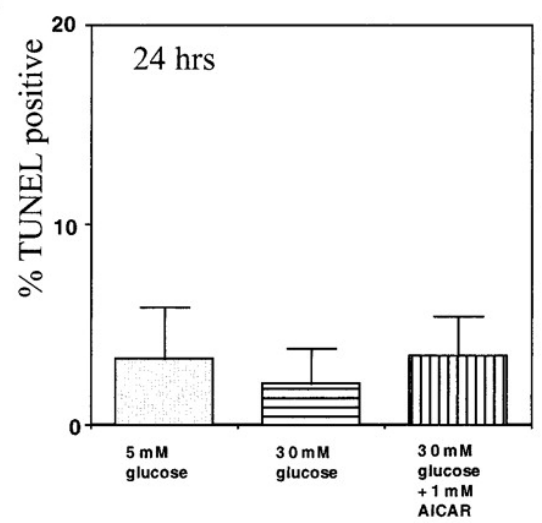

$\mathrm{C}$

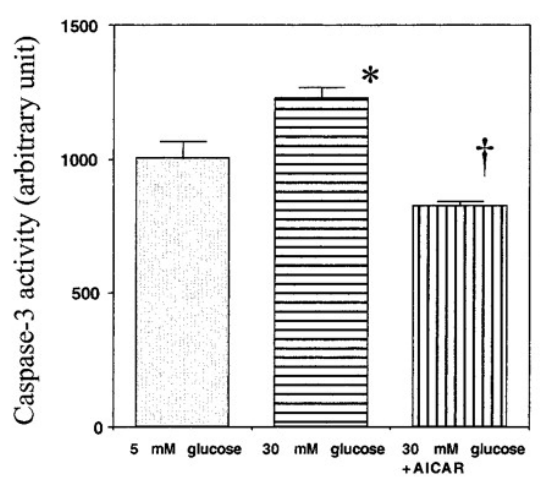

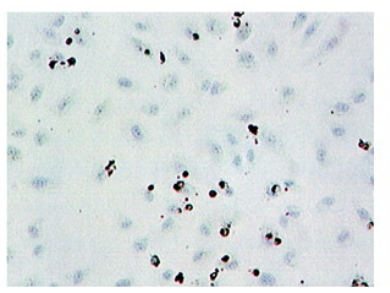

$30 \mathrm{mmol} / \mathrm{l}$ glucose

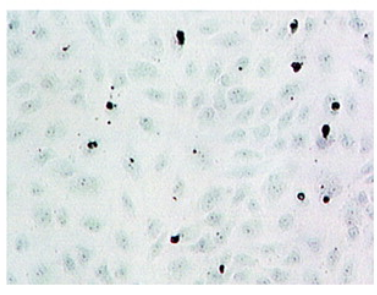

$30 \mathrm{mmol} / \mathrm{l}$ glucose $+1 \mathrm{mmol} / 1$ AICAR
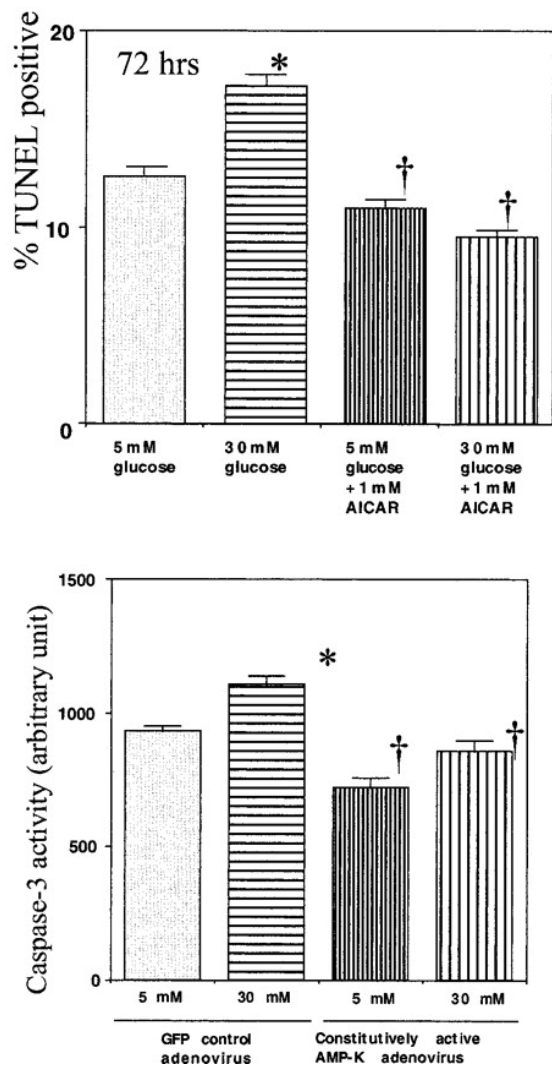

Figure 37 - TUNEL staining will typically show apoptotic configuration among the HUVECs with shrinkage and fragmentation of the nuclei. This figure shows how exposure of HUVECs in a $30 \mathrm{mM}$ hyperglycemic environment caused a significant amount of cell death after 72 hours compared to after only 24 hours. Supporting pictures are also shown, which support greater apoptosis in the $30 \mathrm{mM}$ hyperglycemic environment (permission to reprint from [145]).

Figure 37 also shows the apoptotic effect of 5-aminoimidazole-4-carboxamide-riboside (AICAR) on the HUVECs exposed to the $30 \mathrm{mM}$ hyperglycemic environment. Earlier studies showed that HUVEC incubation with AICAR, which activates AMPK (an enzyme that promotes cellular uptake of glucose), enhanced fatty-acid oxidation in many of the endothelial cells and 
inhibited apoptosis [147, 148, 149]. This indicates that exposure of HUVECs to a hyperglycemic environment induces a negative response by the endothelial cells, which is expected in the experiments of this chapter.

In addition, an earlier study showed that a high oxidative stress level may play a significant role in the pathogenesis of diabetic complications via a hyperglycemic environment causing an increase in oxygen-derived free radicals [150]. Evidence has supported that these reactive molecules play a prominent role as mediators of endothelial cell dysfunction in diabetes [150]. Specifically, the expression of the antioxidant enzymes CuZn-superoxide dismutase, Mnsuperoxide-dismutase, catalase, and glutathione peroxidase were all evaluated after 14 days of culture in a high glucose environment (superoxide dismutase is a class of enzymes that catalyze the separation of superoxide, $\mathrm{O}_{2}^{-}$, into oxygen and hydrogen peroxide [151]). The study demonstrated that a hyperglycemic environment induced an overexpression of these antioxidant enzymes, suggesting that high levels of glucose produce an oxidative stress level in endothelial cells - specifically, HUVECs.

The studies mentioned above are just a small sample of the number of studies researchers have conducted involving the culturing of HUVECs in a high glucose environment. Thus, along with BAECs, researchers have shown the use of HUVECs as a suitable method to study the physiological effects of a hyperglycemic environment on endothelial cells.

\subsubsection{Purpose of This Study}

The purpose of this study was to evaluate BAECs and HUVECs in a normal glycemic (100 mg/dL) and hyperglycemic (400 mg/dL) environment. The hypothesis of this study was that the $400 \mathrm{mg} / \mathrm{dL}$ hyperglycemic cell media solution would evoke a cellular response similar to 
cells exposed to an actual hyperglycemic environment. Therefore, the BAECs and HUVECs exposed to the $400 \mathrm{mg} / \mathrm{dL}$ glycemic cell media solution should exhibit greater glucose consumption over a 72 hour time period than the BAECs and HUVECs exposed to the 100 $\mathrm{mg} / \mathrm{dL}$ normal glycemic cell media solution. In order to measure glucose consumption, the glucose concentration of the glycemic cell media solutions was taken, with an expectation of a decreased concentration over time.

Recall, cells try to maintain a homeostatic balance, and a $100 \mathrm{mg} / \mathrm{dL}$ glycemic environment is considered state of homeostasis. Thus, it was expected that relatively minimal glucose consumption would occur in the normal glycemic environment. Additionally, a 400 $\mathrm{mg} / \mathrm{dL}$ hyperglycemic environment is a state of homeostatic imbalance. Thus, cells should consume higher amounts of glucose to the state of glucose toxicity. The following sections discuss the methods of creating the experiments and the analysis of the accumulated results.

\subsection{Methods}

Three experiments were conducted per cell type - three experiments using BAECs and three experiments using HUVECs. Thus, a total of six experiments were conducted. For each experiment, the endothelial cells were thawed and cultured into 6 wells of a six-well plate using standard cell culture technique, and incubated at $37^{\circ} \mathrm{C}$. During the culture time for the endothelial cells, the two glycemic cell media solutions were prepared and stored at $4{ }^{\circ} \mathrm{C}$ inside the lab refrigerator. Thus, before adding the glycemic media to the cells, it was necessary to warm the solutions in a $37^{\circ} \mathrm{C}$ water bath. Also, since the $400 \mathrm{mg} / \mathrm{dL}$ cell media solutions were made with the unsterile Sigma G6152 D-(+)-Glucose, it was necessary to sterilize it via syringefilter sterilization. The following sections describe the development of each glycemic cell media 
solution, the sterilization of the $400 \mathrm{mg} / \mathrm{dL}$ hyperglycemic cell media solutions, and the incorporation of the glycemic cell media solutions with the endothelial cells.

\subsubsection{Preparation of Glycemic Media Solutions}

The initial preparation for the study involved developing the $400 \mathrm{mg} / \mathrm{dL}$ hyperglycemic cell media solution. For the experiments with BAECs, Sigma G6152 D-(+)-Glucose was added to Lonza Endothelial Cell Basal Media (EBM) (Figure 38). For the HUVEC experiments, Sigma G6152 D-(+)-Glucose was added to Gibco Medium 199.

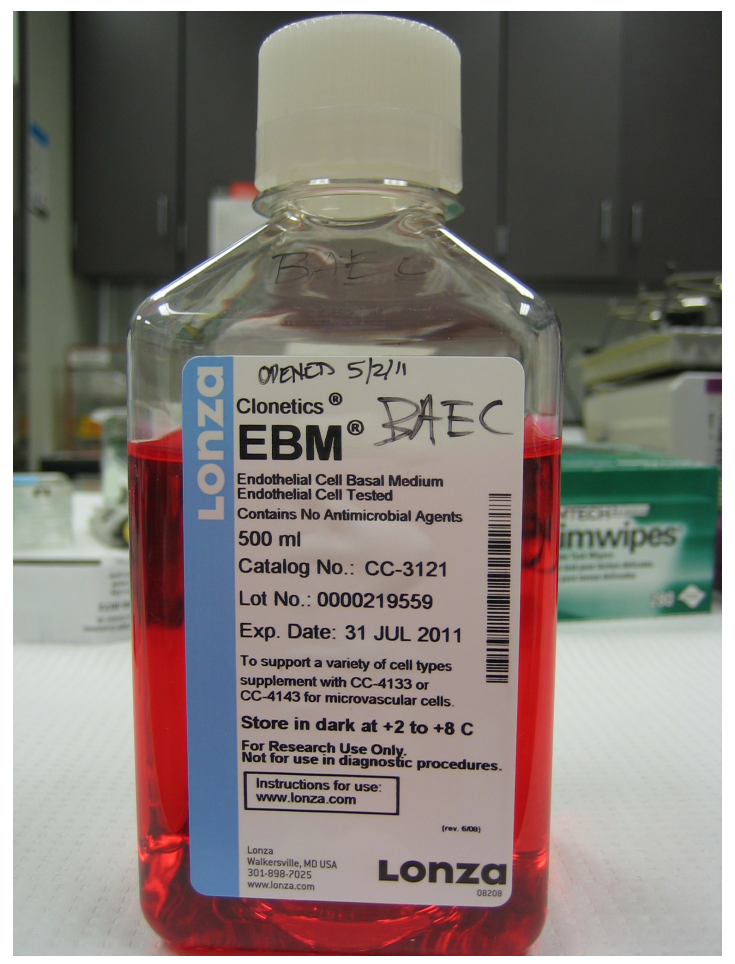

Figure 38 - For experiments with BAECs, the glycemic cell media solutions were made using Lonza Endothelial Cell Basal Medium (EBM).

Recall from Chapter 2, the Gibco Medium 199 was a "low glucose" solution; thus, it already contained $1000 \mathrm{mg} / \mathrm{L}$ of D-glucose $(100 \mathrm{mg} / \mathrm{dL})$. This was also the case for the Lonza EBM. Therefore, neither further modification nor sterilization was necessary for the $100 \mathrm{mg} / \mathrm{dL}$ 
normal glycemic cell media solutions. The following section describes the method used to develop the $400 \mathrm{mg} / \mathrm{dL}$ hyperglycemia cell media solutions for the BAEC and HUVEC experiments.

\subsubsection{Preparation of the $400 \mathrm{mg} / \mathrm{dL}$ Hyperglycemic Cell Media Solution}

The procedure established in Chapter 2 was used to create the $400 \mathrm{mg} / \mathrm{dL}$ hyperglycemic cell media solution for the BAEC and HUVEC experiments. Recall, the method involved adding $x$ g of Sigma G6152 D-(+)-Glucose to be added to $y \mathrm{~mL}$ of cell media. Additionally, it took approximately five hours for the $500 \mathrm{mg} / \mathrm{dL}$ glycemic cell media solution to uniformly mix. Thus, the $400 \mathrm{mg} / \mathrm{dL}$ hyperglycemic cell media solutions for these experiments were mixed for at least six hours on a magnetic hot plate with a magnetic stir bar at $25^{\circ} \mathrm{C}$ and then stored at approximately $4^{\circ} \mathrm{C}$ inside the laboratory refrigerator (Figure 39).

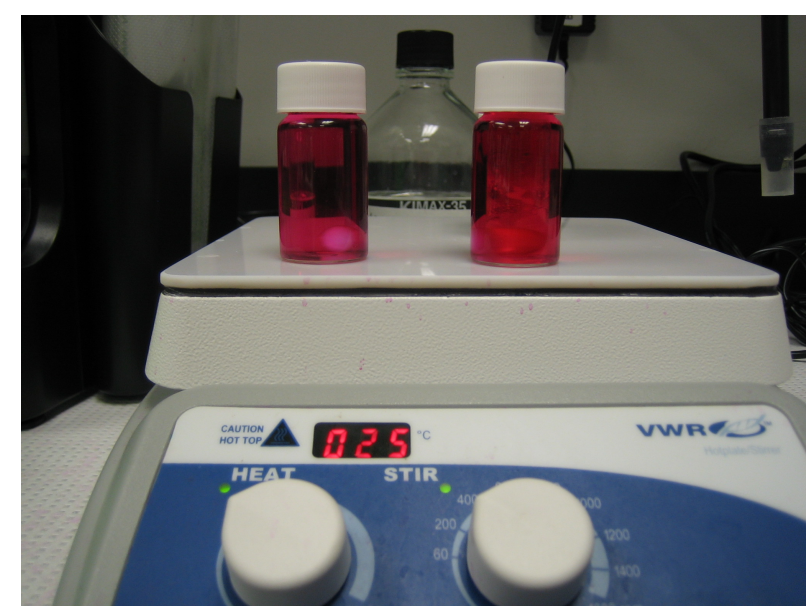

Figure 39 - After adding the Sigma G6152 D-(+)-Glucose to the cell media, the solution was stirred with a magnetic stir plate and bar for at least six hours at $25^{\circ} \mathrm{C}$ to ensure uniform mixing.

Error! Reference source not found.Table 9 below summarizes the recipes used to create the glycemic solutions for the initial four experiments. Since a substantial volume of the $400 \mathrm{mg} / \mathrm{dL}$ hyperglycemic cell media solution was created for the first HUVEC experiment, 
much of it was left over and available for the second experiment with HUVECs. The last two experiments will be discussed later.

Table 9 - Recipes to Create the $400 \mathrm{mg} / \mathrm{dL}$ Hyperglycemic Cell Media Solutions for Initial BAEC \& HUVEC Experiments

\begin{tabular}{|c|c|c|c|c|} 
Experiment & $\begin{array}{c}\text { Volume of Cell } \\
\text { Media }(\mathrm{mL})\end{array}$ & $\begin{array}{c}\text { Amt Glucose } \\
\text { Added (g) }\end{array}$ & $\begin{array}{c}\text { Mixing } \\
\text { Parameters }\end{array}$ & $\begin{array}{c}\text { Recorded } \\
\text { Glucose } \\
\text { Concentration } \\
\text { (mg/dL) }\end{array}$ \\
\hline BAECs 1 & 30 & 0.0906 & $\begin{array}{c}12 \text { hours } \\
25^{\circ} \mathrm{C}\end{array}$ & 432 \\
\hline BAECs 2 & 20 & 0.0613 & $\begin{array}{c}12 \text { hours } \\
25^{\circ} \mathrm{C}\end{array}$ & 435 \\
\hline HUVECs 1 & 40 & 0.1214 & $\begin{array}{c}12 \text { hours } \\
25^{\circ} \mathrm{C}\end{array}$ & 465 \\
\hline HUVECs 2 & 40 & 0.1214 & $\begin{array}{c}12 \text { hours } \\
25^{\circ} \mathrm{C}\end{array}$ & 465 \\
\hline
\end{tabular}

\subsubsection{Sterilization of the $400 \mathrm{mg} / \mathrm{dL}$ Hyperglycemic Cell Media Solutions}

Before adding the $400 \mathrm{mg} / \mathrm{dL}$ hyperglycemic cell media solution to the endothelial cells, it was necessary to sterilize it first to prevent any contamination to the sterile environment of the cells. The method of sterilization used was syringe-filter sterilization with $10 \mathrm{~mL}$ syringes and $0.22 \mu \mathrm{m}$ syringe filters.

Sterilization was conducted in an aseptic environment under a laminar flow hood.

During sterilization, the $10 \mathrm{~mL}$ syringe took up $10 \mathrm{~mL}$ of the hyperglycemic cell media solution (Figure 40a). The $0.22 \mu \mathrm{m}$ syringe filter was placed on the end of the syringe and the $10 \mathrm{~mL}$ of cell media solution was slowly transferred through the filter and into a brand new sterile $50 \mathrm{~mL}$ conical (Figure 40b). A brand new filter was used for every $10 \mathrm{~mL}$ of glycemic media solution being sterilized. The solution in the new $50 \mathrm{~mL}$ conicals was now sterile $400 \mathrm{mg} / \mathrm{dL}$ 
hyperglycemic cell media solution that was ready to be warmed and added to the endothelial cells.
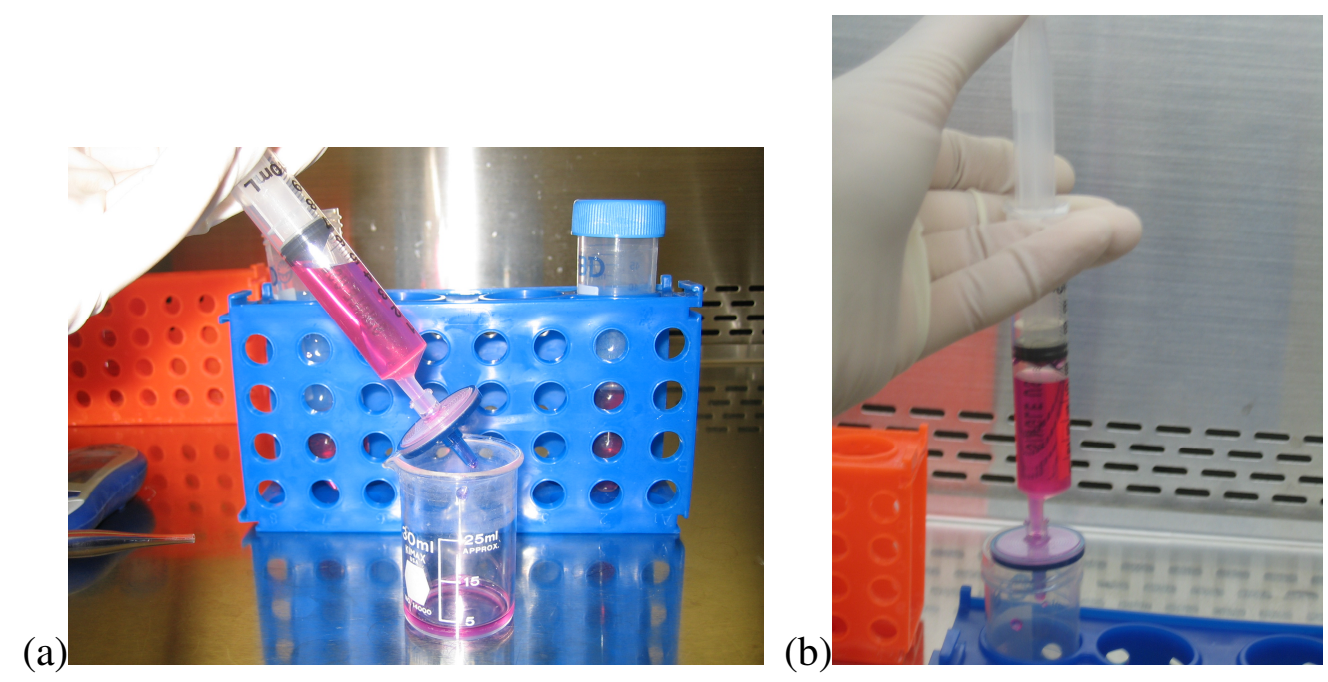

Figure 40 - Syringe-filter sterilization was conducted to sterilize the $400 \mathrm{mg} / \mathrm{dL}$ hyperglycemic cell media solutions. (a) $10 \mathrm{~mL}$ of the solution was taken up by a $10 \mathrm{~mL}$ syringe and the 0.22 $\mu \mathrm{m}$ syringe filter was added onto the end of the syringe. (b) The $10 \mathrm{~mL}$ of solution was slowly transferred into a sterile $50 \mathrm{~mL}$ conical. This entire sterilization was conducted in a laminar flow hood.

\subsubsection{Applying the Glycemic Media Solutions to the Endothelial Cells}

Once sterilization of the $400 \mathrm{mg} / \mathrm{dL}$ hyperglycemic cell media solution was complete, both glycemic solutions were ready to be added to the endothelial cells. The most important factor taken into consideration when working with the endothelial cells was sterility. It was crucial to ensure that, along with the glycemic cell media solutions, the surrounding work environment, the tools, and the hands managing all the equipment were properly sterilized or made aseptic with 70\% isopropyl alcohol (IPA). Therefore, the first step of every experiment was to wash hands with soap and warm water, wear properly fitted latex gloves, and spray the gloves with the 70\% IPA. Next, it was critical to spray the entire inside of the sterile hood with 70\% IPA both before and after the experiment. 
To introduce the glycemic cell media solutions to the cultured cells, an electronic pipette was used with $10 \mathrm{~mL}$ sterile tubes. For each experiment, the $100 \mathrm{mg} / \mathrm{dL}$ normal glycemic cell media solutions were introduced to the cells in the bottom three wells, and the $400 \mathrm{mg} / \mathrm{dL}$ hyperglycemic cell media solutions were introduced to the cells in the top three wells (Figure $41)$.
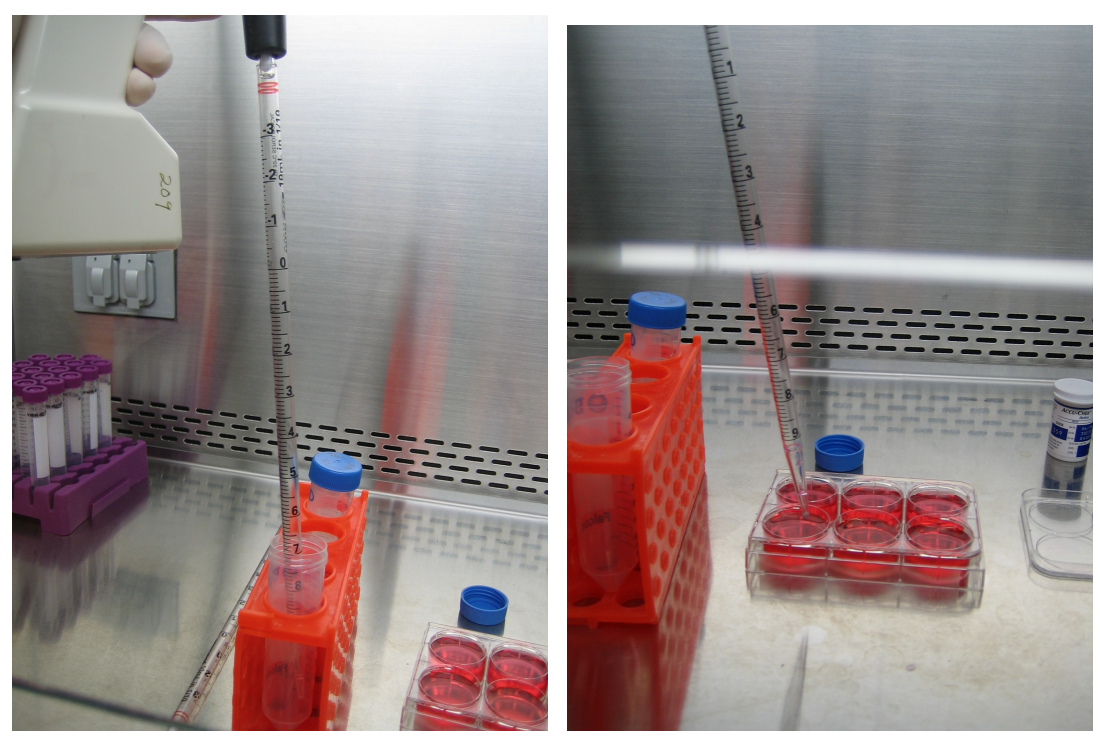

Figure 41 - To introduce the glycemic cell media solutions to the endothelial cells, an electronic pipette was used. The $100 \mathrm{mg} / \mathrm{dL}$ solution was added to the bottom three wells, and the 400 $\mathrm{mg} / \mathrm{dL}$ solution was added to the top three wells.

For the first BAEC experiment, $10 \mathrm{~mL}$ of the $100 \mathrm{mg} / \mathrm{dL}$ and $400 \mathrm{mg} / \mathrm{dL}$ cell media solutions was introduced to each well (Figure 42a). For the second BAEC experiment, and the two HUVEC experiments, the volume of media solution introduced to each well was reduced from $10 \mathrm{~mL}$ to $3 \mathrm{~mL}$ (Figure $42 \mathrm{~b}$ ). The reason for this reduction was to prevent exaggerated glucose toxicity for the endothelial cells and to ensure the media did not overflow from the well. As mentioned before, the method for the third BAEC and HUVEC experiments will be discussed later. 

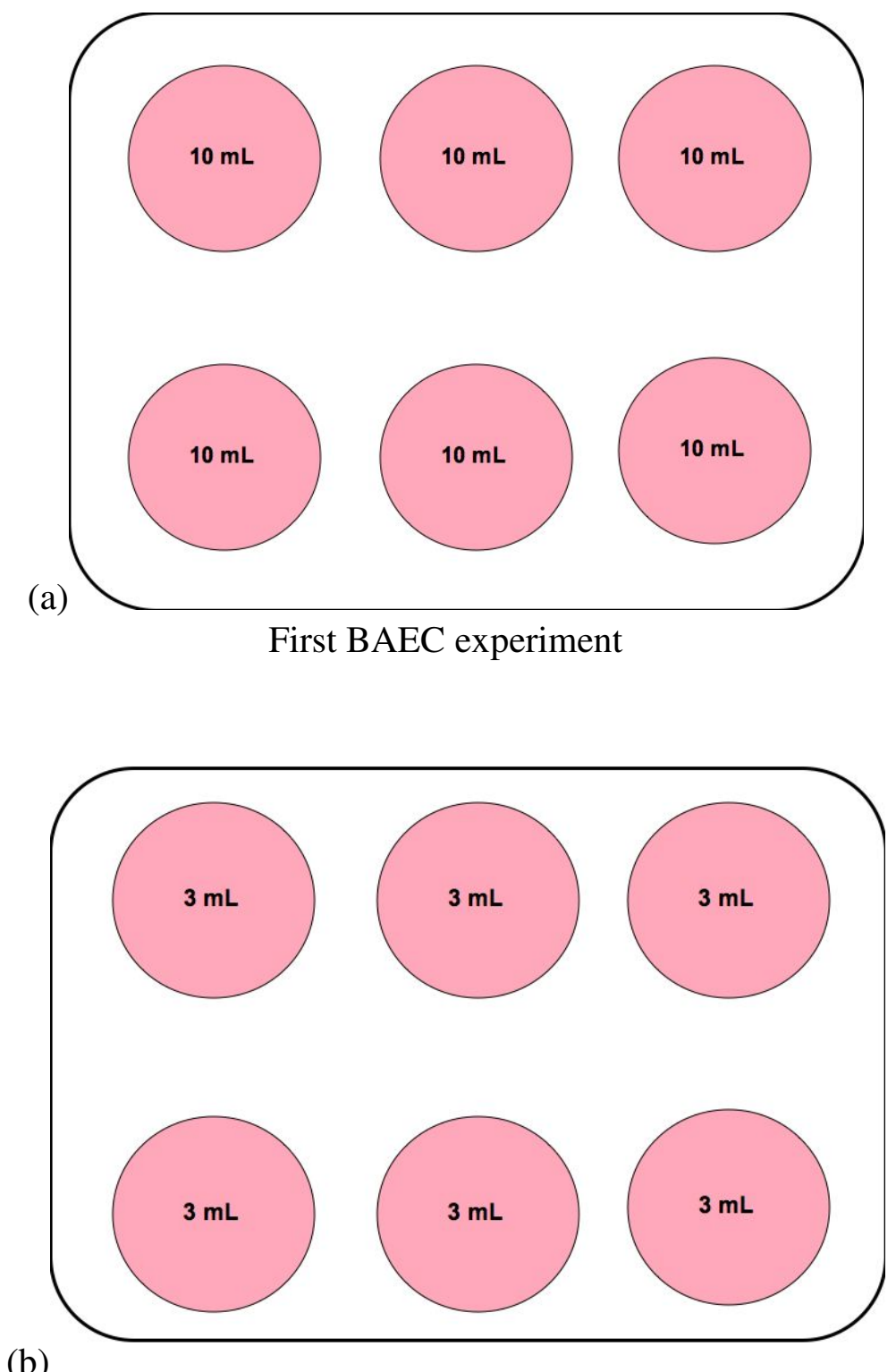

Second BAEC experiment \& first two HUVEC experiments

Figure 42 - (a) For the first BAEC experiment, $10 \mathrm{~mL}$ of the glycemic cell media solutions was added to each well. The $400 \mathrm{mg} / \mathrm{dL}$ cell media was introduced to the cells in the top three wells, and the $100 \mathrm{mg} / \mathrm{dL}$ cell media was introduced to the cells in the bottom three wells. (b) For the second BAEC experiment and the first two HUVEC experiments, the volume of media introduced to each well was reduced to $3 \mathrm{~mL}$. Again, the $400 \mathrm{mg} / \mathrm{dL}$ cell media was introduced to the cells in top three wells, and $100 \mathrm{mg} / \mathrm{dL}$ cell media was introduced to the cells in the bottom three wells. 


\subsubsection{Evaluation of BAECs and HUVECs}

The purpose of this study was to compare and evaluate the glucose consumption and cellular death of BAECs and HUVECs in the hyperglycemic environment. In order to evaluate the glucose consumption, the glucose concentration of the introduced glycemic cell media solution was monitored over the seventy-two hour experimentation time. The glucose concentration of each well was recorded at $\mathrm{t}=0,24,48$, and 72 hours.

In addition to glucose consumption, it was ideal to evaluate cellular morphology, especially of the cells in the $400 \mathrm{mg} / \mathrm{dL}$ hyperglycemic cell media solution to determine if cell death was occurring. Cells in $100 \mathrm{mg} / \mathrm{dL}$ could serve as a control. However, the cells of only the third and final BAEC and HUVEC experiments were evaluated and recorded under a microscope with pictures. It was expected that the wells with the $400 \mathrm{mg} / \mathrm{dL}$ hyperglycemic cell media solutions would have much greater cell death than the wells with the $100 \mathrm{mg} / \mathrm{dL}$ normal glycemic cell media solution, as indicated by a rounded, ball-like morphology.

Once the glycemic cell media solutions were properly introduced to the BAECs and HUVECs, the glucose concentration of each well was recorded with the Aviva Accu-Chek blood glucose meter (the blood glucose meter was sprayed with 70\% IPA before being appended to the aseptic environment under the laminar flow hood). Once the initial $\mathrm{t}=0$ evaluation was complete, the cells were stored in the incubator. Again at $\mathrm{t}=24,48$, and 72 hours, the cells of each well were re-evaluated. The following section explains the recorded results for each of the first two BAEC and HUVEC experiments. In addition, the following section will lead into the reason and results of the third and final BAEC and HUVEC experiments. 


\subsection{Results}

This section displays the accumulated data of the first two BAEC and HUVEC experiments and is formatted to assess the results between the cells exposed to the $100 \mathrm{mg} / \mathrm{dL}$ normal glycemic cell media solutions and the cells exposed to the $400 \mathrm{mg} / \mathrm{dL}$ hyperglycemic cell media solutions.

\subsection{1 $100 \mathrm{mg} / \mathrm{dL}$ Normal Glycemic Environment}

\subsubsection{Glucose Consumption}

Recall from Chapter 1, the average blood glucose level of a healthy individual falls within the $80-120 \mathrm{mg} / \mathrm{dL}$ range. The initial expectation for the endothelial cells exposed to the $100 \mathrm{mg} / \mathrm{dL}$ solution was for there to be minimal glucose consumption since the cells are in a state of homeostasis. Therefore, the results should show minimal decrease in glucose concentration throughout the entire 72 hour test period.

Figure 43 shows the average glucose concentrations of all wells exposed to the 100 $\mathrm{mg} / \mathrm{dL}$ normal glycemic cell media solution at each time of evaluation. Additionally, Table 10 lists the average glucose concentrations for each experiment, as well as the calculated difference in glucose concentration between $\mathrm{t}=0$ and $\mathrm{t}=72$ hours. 


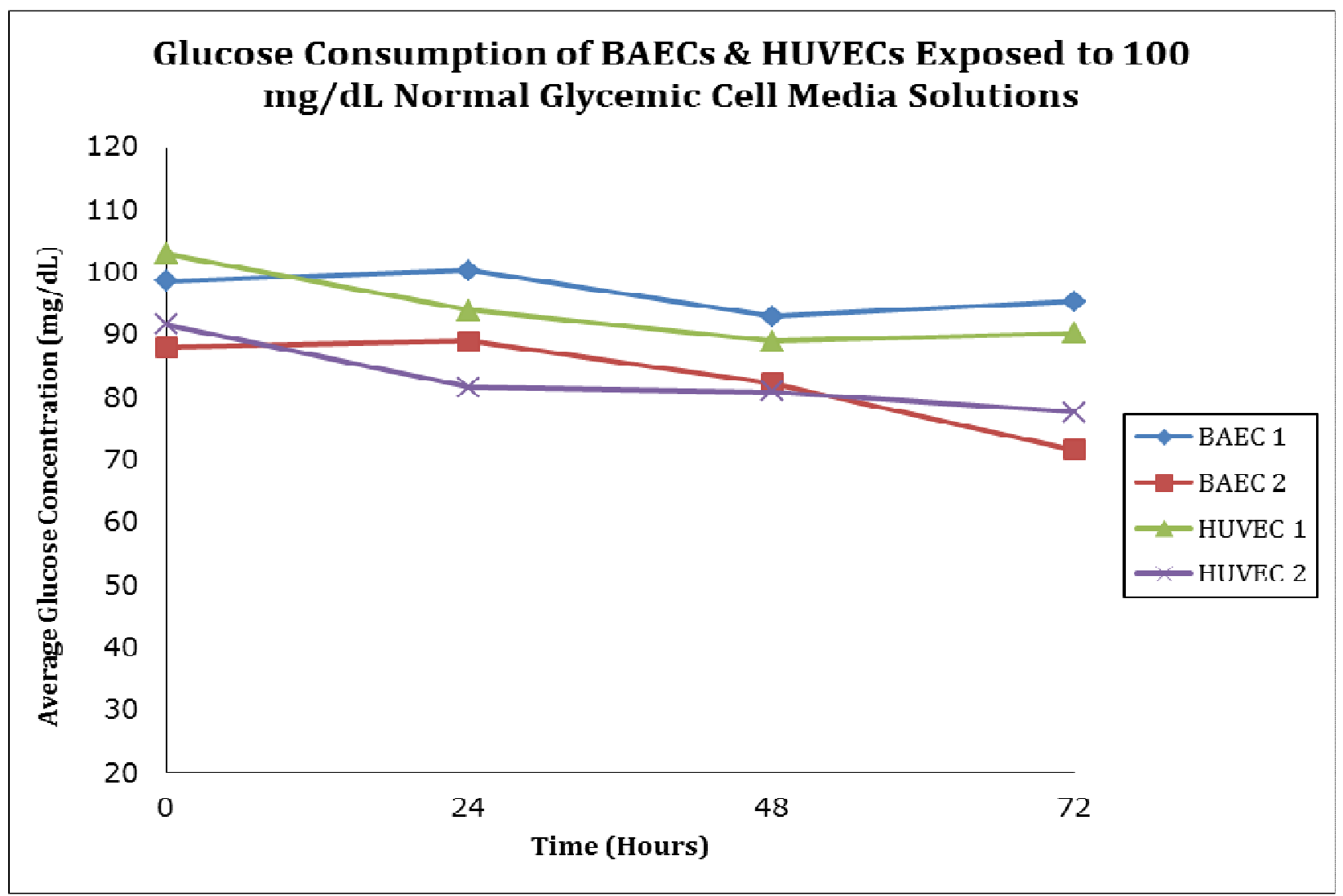

Figure 43 - There was minimal decrease in glucose concentration throughout the 72 hour test period; therefore, the glucose consumption for the BAEC and HUVEC studies was minimal.

Table 10 - Average Glucose Concentrations of 100 mg/dL Normal Glycemic Cell Media Solutions Exposed to BAECs \& HUVECs

\begin{tabular}{|c|c|c|c|c|c|c|}
\hline \multicolumn{6}{|c|}{ Average Glucose Concentrations of the 100 mg/dL Normal Glycemic Cell Media } \\
Solutions Exposed to BAECs \& HUVECs \\
\hline \multirow{2}{*}{$\begin{array}{c}\text { Time } \\
\text { (Hours) }\end{array}$} & \multicolumn{5}{|c|}{ Average Glucose Concentration of Three 100 mg/dL Wells } \\
\cline { 2 - 7 } & BAECs 1 & BAECs 2 & HUVECs 1 & HUVECs 2 & Average & St. Dev. \\
\hline $\mathbf{0}$ & 98.67 & 88.00 & 103.00 & 91.67 & 95.33 & 6.76 \\
\hline $\mathbf{2 4}$ & 100.33 & 89.00 & 94.00 & 81.67 & 91.25 & 6.83 \\
\hline $\mathbf{4 8}$ & 93 & 82.33 & 89 & 81 & 86.33 & 4.90 \\
\hline $\mathbf{7 2}$ & 95.33 & 71.67 & 90.33 & 77.67 & 83.75 & 9.49 \\
\hline$\Delta(\mathbf{t 0 - t 7 2 )}$ & 3.33 & 16.33 & 12.67 & 14.00 & 11.58 & 5.70 \\
\hline
\end{tabular}


Looking at Figure 43, the glucose consumption for the BAEC and HUVEC trials looked minimal; thus, showing that there was little glucose consumption by the endothelial cells exposed to the normal glycemic solution. The values recorded in Table 10 were calculated by taking the average of the recorded glucose concentrations for all three wells containing the 100 $\mathrm{mg} / \mathrm{dL}$ normal glycemic cell media solution at each evaluation period (these values can be seen in Appendix B). In addition, Table 10 gives the average of these averages, as well as the standard deviation, in order to illustrate the low degree of variance in the accumulated data.

In order to analyze whether an average $\Delta_{(\mathrm{t} 0-\mathrm{t} 72)}$ of $11.58 \mathrm{mg} / \mathrm{dL}$ was a significant reduction in glucose concentration, a series of two-tailed T-tests were conducted on the recorded glucose concentrations of each well for each experiment between each time of evaluation. The ttests were conducted assuming that the recorded data was normally distributed. Table 11 shows the calculated $p$-values for each T-test with the null hypothesis $H_{0}: \mu_{1}=\mu_{2}$, the alternative hypothesis $H_{a}: \mu_{1} \neq \mu_{2}$, and $\alpha=0.05$.

Table 11 - Two-Tailed T-Tests Results for BAECs \& HUVECs Exposed to $100 \mathrm{mg} / \mathrm{dL}$ Normal Glycemic Cell Media Solutions

\begin{tabular}{|c|c|c|}
\hline \multicolumn{3}{|c|}{$H_{0}: \mu_{1}=\mu_{2} ; H_{a}: \mu_{1} \neq \mu_{2} ; \alpha=0.05$} \\
\hline Array 1 (hours) & Array 2 (hours) & $p$-Value \\
\hline 0 & 24 & 0.165750622 \\
\hline 0 & 48 & 0.00129592 \\
\hline $\mathbf{0}$ & 72 & 0.002858474 \\
\hline 24 & 48 & 0.080847457 \\
\hline 24 & 72 & 0.050738757 \\
\hline 48 & 72 & 0.444784919 \\
\hline
\end{tabular}


Table 11 shows a statistically significant difference between the average glucose concentrations for $\mathrm{t}=0$ and $\mathrm{t}=72$ hours. The question turned to how this difference compared with the difference seen in the $400 \mathrm{mg} / \mathrm{dL}$ hyperglycemic environment. Therefore, it was essential to review the data recorded from the endothelial cells exposed to the $400 \mathrm{mg} / \mathrm{dL}$ hyperglycemic cell media solutions, and compare the amount of glucose consumption between the two environments.

\subsection{2 $400 \mathrm{mg} / \mathrm{dL}$ Hyperglycemic Environment}

\subsubsection{Glucose Consumption}

Figure 44 shows the average glucose concentrations of all wells exposed to the 400 $\mathrm{mg} / \mathrm{dL}$ hyperglycemic cell media solution at each time of evaluation. (Note: the reason that $\mathrm{t}=0$ does not closely correspond to $400 \mathrm{mg} / \mathrm{dL}$ will be discussed later.) Additionally, Table 12 lists the average glucose concentrations for each experiment, as well as the calculated difference in glucose concentration between $\mathrm{t}=0$ and $\mathrm{t}=72$ hours. 


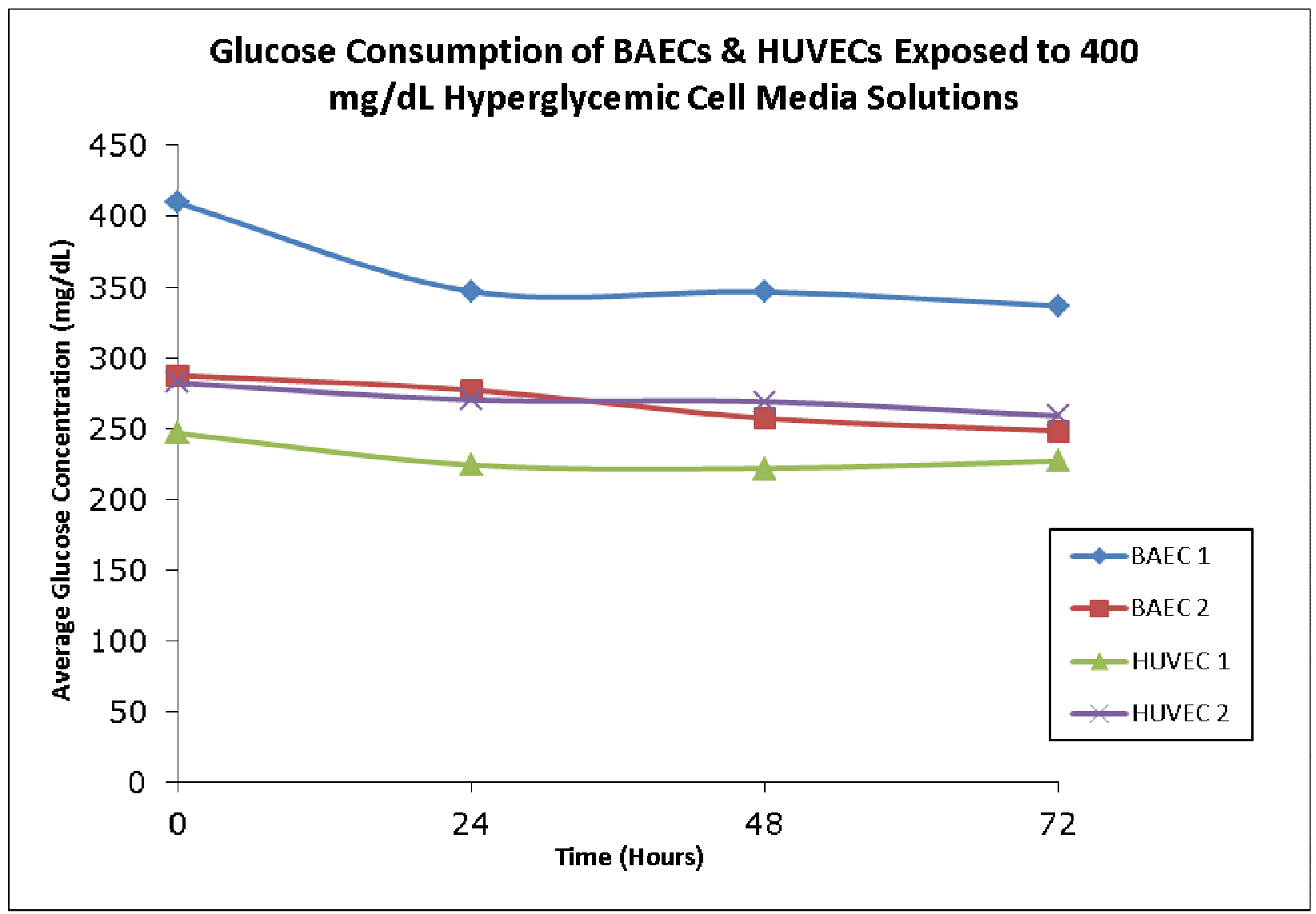

Figure 44 - There was a greater decrease in glucose concentration throughout the 72 hour test period. The data also shows that there was a substantial decrease in glucose concentration for the second BAEC experiment, as well as the two HUVEC experiments starting at $\mathrm{t}=0$. 
Table 12 - Average Glucose Concentrations of 400 mg/dL Hyperglycemic Cell Media Solutions Exposed to BAECs \& HUVECs

\begin{tabular}{|c|c|c|c|c|c|c|}
\hline \multicolumn{6}{|c|}{ Average Glucose Concentrations of the 400 mg/dL Hyperglycemic Cell Media } \\
Solutions Exposed to BAECs \& HUVECs \\
\hline \multirow{2}{*}{$\begin{array}{c}\text { Time } \\
\text { Hours) }\end{array}$} & \multicolumn{6}{|c|}{ Average Glucose Concentration of Three 400 mg/dL Wells } \\
\cline { 2 - 7 } & BAECs 1 & BAECs 2 & HUVECs 1 & HUVECs 2 & Average & St. Dev. \\
\hline $\mathbf{0}$ & 409.33 & 287.67 & 246.67 & 282.33 & 306.50 & 70.93 \\
\hline $\mathbf{2 4}$ & 347.00 & 277.33 & 224.33 & 270.33 & 279.75 & 50.62 \\
\hline $\mathbf{4 8}$ & 346.67 & 257.33 & 222.00 & 269.00 & 273.75 & 52.56 \\
\hline $\mathbf{7 2}$ & 336.67 & 248.33 & 227.00 & 259.00 & 267.75 & 47.83 \\
\hline$\Delta$ (t0-t72) & 72.67 & 39.33 & 19.67 & 23.33 & 38.75 & 24.17 \\
\hline
\end{tabular}

The initial expectation for the BAECs and HUVECs exposed to the $400 \mathrm{mg} / \mathrm{dL}$

hyperglycemic cell media solutions was for a high level of glucose consumption over a 72 hour period. Looking at Figure 44, there was a greater decrease in glucose concentration over the 72 hour time period. The values in Table 12 were calculated by taking the average of the recorded glucose concentrations for all three wells containing the $400 \mathrm{mg} / \mathrm{dL}$ hyperglycemic cell media solution at each evaluation period (these values can also be seen in Appendix B).

The average $\Delta_{(\mathrm{t} 0-\mathrm{t} 72)}$ of the glucose concentration in the cells was $38.75 \mathrm{mg} / \mathrm{dL}$ - a substantial amount of glucose consumption. In order to analyze whether the average $\Delta$ (t0-t72) $_{\text {of }}$ $38.75 \mathrm{mg} / \mathrm{dL}$ was a significant reduction in glucose concentration, a series of two-tailed t-tests was conducted on the recorded glucose concentrations of each well for each experiment between each time of evaluation. These tests were conducted assuming that the recorded data was normally distributed. Table 13 shows the calculated $p$-values for each t-test with a null hypothesis $H_{0}: \mu_{1}=\mu_{2}$, an alternative hypothesis $H_{a}: \mu_{1} \neq \mu_{2}$, and $\alpha=0.05$. 
Table 13 - Two-Tailed T-Tests Results for BAECs \& HUVECs Exposed to $400 \mathrm{mg} / \mathrm{dL}$ Hyperglycemic Cell Media Solutions

\begin{tabular}{|c|c|c|}
\hline \multicolumn{3}{|c|}{$H_{0}: \mu_{1}=\mu_{2} ; H_{a}: \mu_{1} \neq \mu_{2} ; \alpha=0.05$} \\
\hline Array 1 (hours) & Array 2 (hours) & $p$-Value \\
\hline 0 & 24 & 0.264684404 \\
\hline 0 & 48 & 0.176583635 \\
\hline $\mathbf{0}$ & 72 & 0.102422521 \\
\hline 24 & 48 & 0.761585943 \\
\hline 24 & 72 & 0.526862047 \\
\hline 48 & 72 & 0.752230261 \\
\hline
\end{tabular}

Although the average $\Delta_{(\mathrm{t} 0-\mathrm{t} 72)}$ was approximately $38.75 \mathrm{mg} / \mathrm{dL}$, the calculated $p$-values showed no statistical significant difference between any average glucose concentrations at any evaluation points - especially between $\mathrm{t}=0$ and $\mathrm{t}=72$ hours.

Additionally, there was cause for concern in the results. The expected $t=0$ glucose concentration of approximately $400 \mathrm{mg} / \mathrm{dL}$ was recorded for the first BAEC experiment. However, the data shows a substantial decrease in glucose concentration at $t=0$ for the second BAEC experiment, and the two HUVEC experiments. This observation will be discussed and explored in the following sub-sections. 


\subsection{Investigating the Decreased Glucose Concentration at $\mathbf{t}=\mathbf{0}$ for the $400 \mathrm{mg} / \mathrm{dL}$ hyperglycemic cell media solutions}

\subsubsection{Testing the Loss of Glucose during Syringe-Filter Sterilization}

Recall from Section 3.2.3, sterilization of the $400 \mathrm{mg} / \mathrm{dL}$ hyperglycemic cell media solutions was necessary before introducing the solutions to the endothelial cells. The method of sterilization used was syringe-filter sterilization with $10 \mathrm{~mL}$ syringes and $0.22 \mu \mathrm{m}$ syringe filters. A significant step missed in the procedure was that the glucose concentration of the solutions was not taken right after sterilization. This would have been useful, since the glucose concentration of the solution was going to be recorded after it was added to the cells.

A potential cause for the initial loss of glucose was thought to be the syringe-filter sterilization. Remember, an extremely small amount of glucose can differentiate a hyperglycemic glucose concentration from a normal glycemic concentration; therefore, even a minuscule amount of glucose lost in the $0.22 \mu \mathrm{m}$ filters could cause the glucose concentration of the $400 \mathrm{mg} / \mathrm{dL}$ hyperglycemic solutions to drop to roughly $250 \mathrm{mg} / \mathrm{dL}$ - as seen in the second BAEC experiment and the two HUVEC experiments.

In order to test this hypothesis, four sets of $400 \mathrm{mg} / \mathrm{dL}$ hyperglycemic cell media solutions were created and sterilized into three separate $10 \mathrm{~mL}$ samples per solution (a total of twelve test samples). From previously derived calculations, approximately $0.12 \mathrm{~g}$ of Sigma G6152 D-(+)-Glucose was added to $40 \mathrm{~mL}$ of Gibco Cell Medium 199. Table 14 displays the exact amounts of glucose added to the volume of cell media. 
Table 14 - Recipes to Create 400 mg/dL Hyperglycemic Solutions to Test Glucose Loss during Syringe-Filter Sterilization

\begin{tabular}{|c|c|c|}
\hline Solution Sample & Glucose Added $(\mathrm{g})$ & Volume of Cell Media $(\mathrm{mL})$ \\
\hline $\mathbf{1}$ & 0.1213 & 40 \\
\hline $\mathbf{2}$ & 0.1208 & 40 \\
\hline $\mathbf{3}$ & 0.1209 & 40 \\
\hline $\mathbf{4}$ & 0.1211 & 40 \\
\hline
\end{tabular}

Each solution was mixed on a magnetic hot plate with a magnetic stir bar at $25^{\circ} \mathrm{C}$ for at least six hours. Once the solutions were uniformly mixed, the glucose concentration was taken with the Aviva Accu-Chek blood glucose meter. Next, the solutions were sterilized and transferred into twelve different sterile $50 \mathrm{~mL}$ conicals. The glucose concentration was measured again for each sample and the amount of glucose loss was calculated and recorded in Table 15. The calculations were conducted by converting the post sterilization glucose concentration into grams per volume of cell media. Additionally, the calculated glucose loss was based off the initial amount of glucose added to the solutions, not the initial glucose concentration of each solution; thus, if the calculation theoretically indicated no glucose loss, then it was not included in the data. 
Table 15 - Effect of Syringe-Filter Sterilization on 400 mg/dL Hyperglycemic Cell Media Solutions

\begin{tabular}{|c|c|c|c|c|c|}
\hline \multicolumn{6}{|c|}{$\begin{array}{c}\text { Effect of Syringe-Filter Sterilization on } 400 \mathrm{mg} / \mathrm{dL} \text { Hyperglycemic Cell Media } \\
\text { Solutions }\end{array}$} \\
\hline Solution \# & $\begin{array}{l}\text { Amount of } \\
\text { Glucose in } \\
\text { Solution (g) }\end{array}$ & $\begin{array}{l}\text { Initial Glucose } \\
\text { Concentration } \\
\quad(\mathbf{m g} / \mathbf{d L})\end{array}$ & $\begin{array}{c}\text { Test } \\
\text { Sample \# }\end{array}$ & $\begin{array}{c}\text { Post- } \\
\text { Sterilization } \\
\text { Glucose } \\
\text { Concentration } \\
\text { (mg/dL) }\end{array}$ & $\begin{array}{l}\text { Glucose } \\
\text { Lost (g) }\end{array}$ \\
\hline \multirow{3}{*}{1} & \multirow{3}{*}{0.1613} & \multirow{3}{*}{416} & 1 & 387 & 0.0065 \\
\hline & & & 2 & 412 & N/A \\
\hline & & & 3 & 402 & 0.0005 \\
\hline \multirow{3}{*}{2} & \multirow{3}{*}{0.1608} & \multirow{3}{*}{411} & 1 & 403 & N/A \\
\hline & & & 2 & 392 & 0.004 \\
\hline & & & 3 & 406 & N/A \\
\hline \multirow{3}{*}{3} & \multirow{3}{*}{0.1609} & \multirow{3}{*}{424} & 1 & 403 & N/A \\
\hline & & & 2 & 390 & 0.0049 \\
\hline & & & 3 & 396 & 0.0025 \\
\hline \multirow{3}{*}{4} & \multirow{3}{*}{0.1611} & \multirow{3}{*}{429} & 1 & 395 & 0.0031 \\
\hline & & & 2 & 374 & 0.0015 \\
\hline & & & 3 & 405 & N/A \\
\hline \multicolumn{4}{|c|}{ Average } & 397.08 & 0.0033 \\
\hline \multicolumn{4}{|c|}{ Standard Deviation } & 10.33 & 0.002 \\
\hline
\end{tabular}

Table 15 shows that all four solutions experienced a very small loss of glucose after syringe-filter sterilization with an average glucose loss of $0.0033 \mathrm{~g}$. Thus, the experiment suggested that the root cause of the low glucose concentrations recorded at $t=0$ was not the sterilization process. However, a third set of experiments with BAECs and HUVECs was conducted with an additional amount of glucose added to the initial calculated mass of glucose for the $400 \mathrm{mg} / \mathrm{dL}$ hyperglycemic solution recipe. This compensation was conducted in the attempt to limit the degree of glucose loss in the hyperglycemic solutions. The following section describes these experiments. 


\subsubsection{Third BAEC \& HUVEC Experiment}

\subsubsection{Preparation and Application of Glycemic Cell Media Solutions}

For the $400 \mathrm{mg} / \mathrm{dL}$ hyperglycemic solutions of the third BAEC and HUVEC experiments, an additional $0.020 \mathrm{~g}$ of glucose was added to the calculated amount of Sigma G6152 D-(+)Glucose to compensate for any lost glucose during syringe-filter sterilization. Recall, Lonza EBM was used for the BAEC experiment and Gibco Medium 199 was used for the HUVEC experiment. Table 16 summarizes the recipes of the hyperglycemic solutions for each experiment.

Table 16 - Recipes to Create 400 mg/dL Hyperglycemic Solutions for Final BAEC \& HUVEC Experiments

\begin{tabular}{|c|c|c|c|c|} 
Experiment & $\begin{array}{c}\text { Volume of Cell } \\
\text { Media }(\mathrm{mL})\end{array}$ & $\begin{array}{c}\text { Amt Glucose } \\
\text { Added }(\mathrm{g})\end{array}$ & $\begin{array}{c}\text { Mixing } \\
\text { Parameters }\end{array}$ & $\begin{array}{c}\text { Recorded } \\
\text { Glucose } \\
\text { Concentration } \\
\text { (mg/dL) }\end{array}$ \\
\hline BAECs 3 & 20 & 0.0813 & $\begin{array}{c}12 \text { hours } \\
25^{\circ} \mathrm{C}\end{array}$ & 461 \\
\hline HUVECs 3 & 20 & 0.0807 & $\begin{array}{c}12 \text { hours } \\
25^{\circ} \mathrm{C}\end{array}$ & 519 \\
\hline
\end{tabular}

In addition, the amount of glycemic cell media solution added to each well was increased from $3 \mathrm{~mL}$ to $6 \mathrm{~mL}$ per well (Figure 45). The results of these experiments are discussed in the following section. 


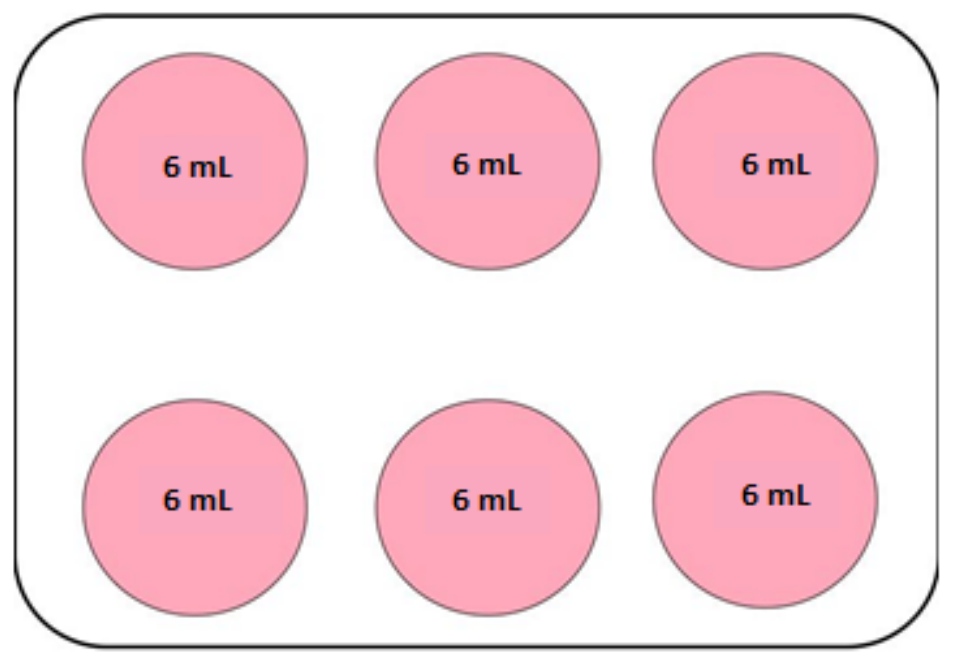

Figure 45- Amount of glycemic cell media solution added to each well for the third BAEC \& HUVEC experiments

\subsection{Results of Third BAEC \& HUVEC Experiments}

\subsection{1 $100 \mathrm{mg} / \mathrm{dL}$ Normal Glycemic Environment}

\subsubsection{Glucose Consumption}

Figure 46 shows the average glucose concentrations of all wells exposed to the 100 mg/dL normal glycemic cell media solution at each time of evaluation. Additionally, Table 17 lists the average glucose concentrations for each experiment, as well as the calculated difference in glucose concentration between $\mathrm{t}=0$ and $\mathrm{t}=72$ hours. 


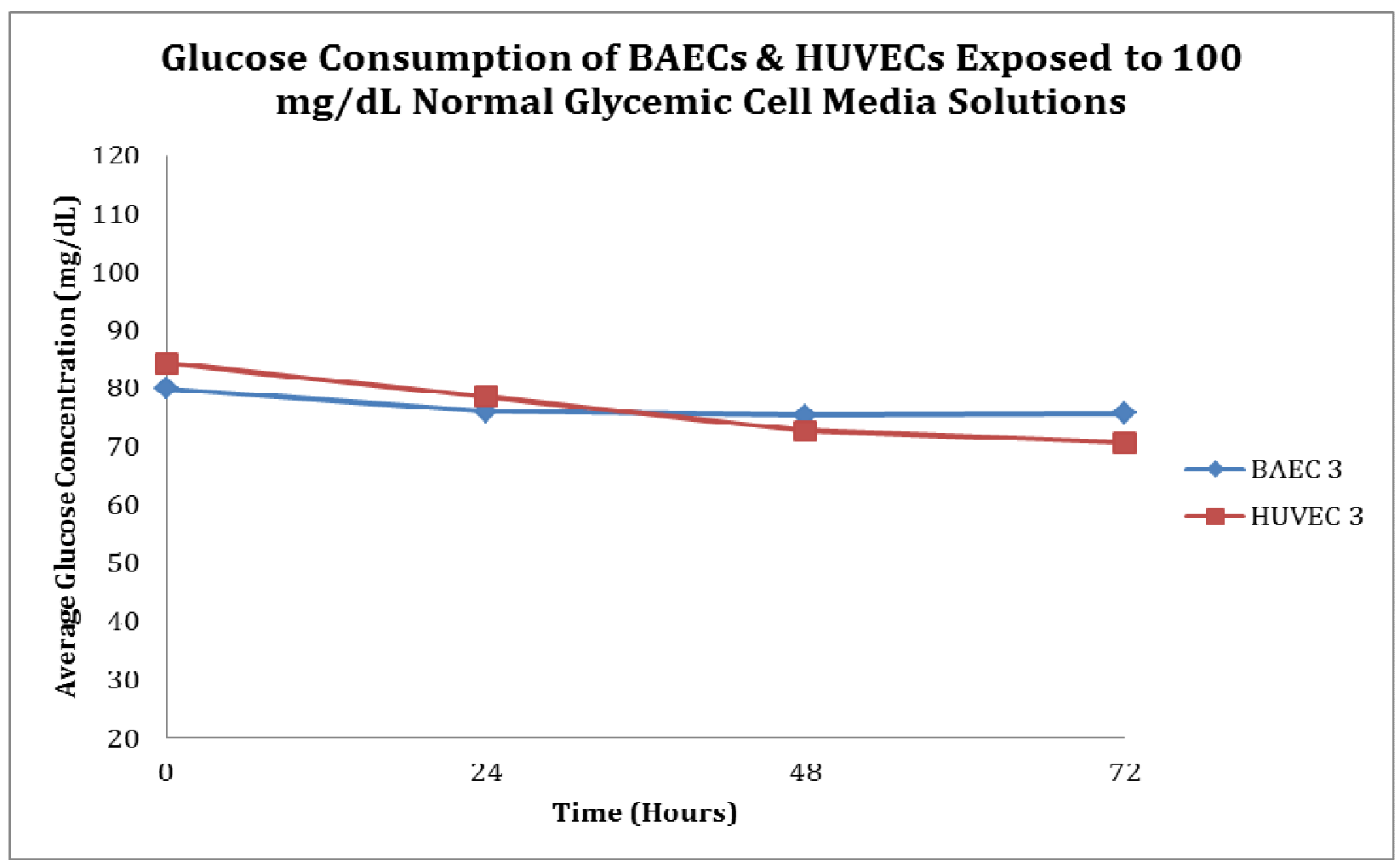

Figure 46 - The BAECs and HUVECs exposed to the $100 \mathrm{mg} / \mathrm{dL}$ normal glycemic cell media solutions in the third experiment exhibited similar decrease in glucose concentration.

Table 17 - Average Glucose Concentrations of the $100 \mathrm{mg} / \mathrm{dL}$ Normal Glycemic Cell Media Solutions Exposed to BAECs \& HUVECs of Final Experiments

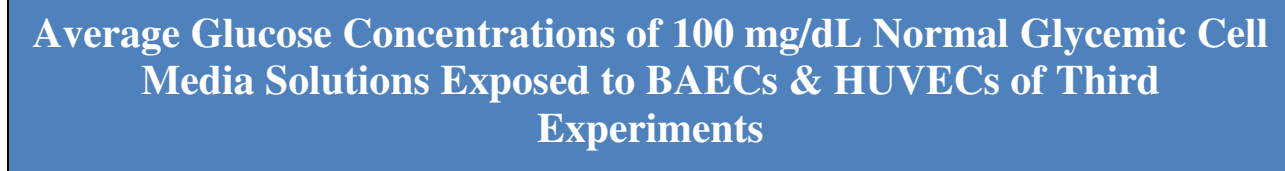

\begin{tabular}{|c|c|}
\hline & Average Glucose Concentration of Three $100 \mathrm{mg} / \mathrm{dL}$ \\
Wells
\end{tabular}

\begin{tabular}{|c|c|c|c|c|}
\hline \multirow{2}{*}{ Time (Hours) } & \multicolumn{4}{|c|}{ Cells } \\
\cline { 2 - 5 } & BAEC 3 & HUVEC 3 & Average & St. Dev. \\
\hline $\mathbf{0}$ & 80.00 & 84.33 & 82.17 & 3.06 \\
\hline $\mathbf{4 4}$ & 76.00 & 78.67 & 77.33 & 1.88 \\
\hline $\mathbf{4 2}$ & 75.33 & 72.67 & 74 & 1.88 \\
\hline$\Delta_{(\mathbf{t 0 - t 7 2 )}}$ & 75.67 & 70.67 & 73.17 & 3.53 \\
\hline & 4.33 & 13.67 & 9.00 & 6.599 \\
\hline
\end{tabular}


As expected, Figure 46 shows the same decrease in glucose concentration that the previous experiments exhibited for the endothelial cells exposed to the $100 \mathrm{mg} / \mathrm{dL}$ normal glycemic cell media solutions. In addition, Table 17 shows the little change in glucose concentration from $\mathrm{t}=0$ hours to $\mathrm{t}=72$ hours $-9.00 \mathrm{mg} / \mathrm{dL}$.

The series of two-tailed T-tests were conducted in order to analyze whether or not there was a statistically significant difference between the average glucose concentration of the wells at $\mathrm{t}=0$ and $\mathrm{t}=72$ hours (Table 18).

Table 18 - Two-Tailed T-Test Results for the Final BAEC \& HUVEC Experiments Exposed to $100 \mathrm{mg} / \mathrm{dL}$ Normal Glycemic Cell Media Solutions

\begin{tabular}{|c|c|c|}
\hline $\begin{array}{c}\text { Two-Tailed T-Tests Results for the Third BAEC \& HUVEC Experiments Exposed } \\
\text { to } 100 \mathrm{mg} / \mathrm{dL} \text { Normal Glycemic Cell Media Solutions }\end{array}$ \\
\hline \multicolumn{3}{|c|}{$\mathbf{H}_{\mathbf{0}}: \boldsymbol{\mu}_{1}=\boldsymbol{\mu}_{2} ; \mathrm{H}_{\mathrm{a}}: \boldsymbol{\mu}_{1} \neq \boldsymbol{\mu}_{2} ; \boldsymbol{\alpha}=\mathbf{0 . 0 5}$} \\
\hline Array $\mathbf{1}$ (hours) & Array 2 (hours) & $\boldsymbol{p}$-Value \\
\hline 0 & 24 & 0.097796746 \\
\hline 0 & 48 & 0.010774561 \\
\hline $\mathbf{0}$ & $\mathbf{7 2}$ & $\mathbf{0 . 0 0 6 7 2 1 3 9 3}$ \\
\hline 24 & 48 & 0.255720974 \\
\hline 24 & 72 & 0.166718562 \\
\hline 48 & 72 & 0.769038752 \\
\hline
\end{tabular}

The statistical analysis shows a statistically significant difference between the average glucose concentration at $t=0$ hours and $t=72$ hours. As before, this difference was compared to the statistical difference seen between the average glucose concentration at $\mathrm{t}=0$ and $\mathrm{t}=72$ hours for the endothelial cells exposed to the $400 \mathrm{mg} / \mathrm{dL}$ hyperglycemic cell media solutions. 


\subsubsection{Cell Confluency}

For the third BAEC \& HUVEC experiments, cell morphology was observed at each time of evaluation. Studies have shown that hyperglycemia can induce cellular apoptosis in HUVECs and BAECs $[145,152]$; thus, the BAECs and HUVECs were observed under a microscope to see if cell confluency had decreased over the 72 hour experimentation time. Since the BAECs and HUVECs exposed to the $100 \mathrm{mg} / \mathrm{dL}$ normal glycemic cell media solutions were generally in a state of homeostasis, a significant decrease in cell confluency was not expected.

Figure 47 shows that the HUVEC (Figure 47a) and BAEC (Figure 47b) cell confluency did not decrease from $t=0$ hours to $t=72$ hours (HUVEC confluency was also initially much better than BAEC confluency). 
(a)

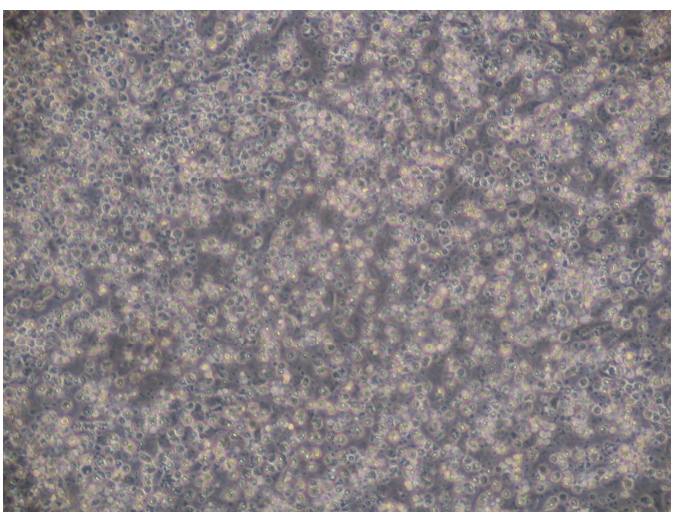

$\mathrm{t}=0$

(b)

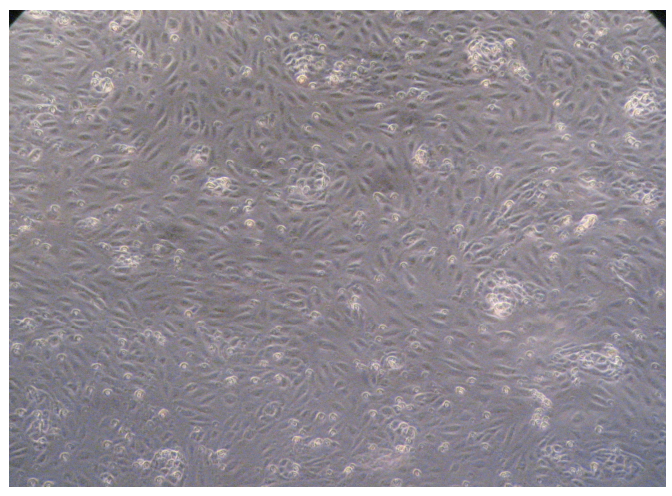

$\mathrm{t}=0$

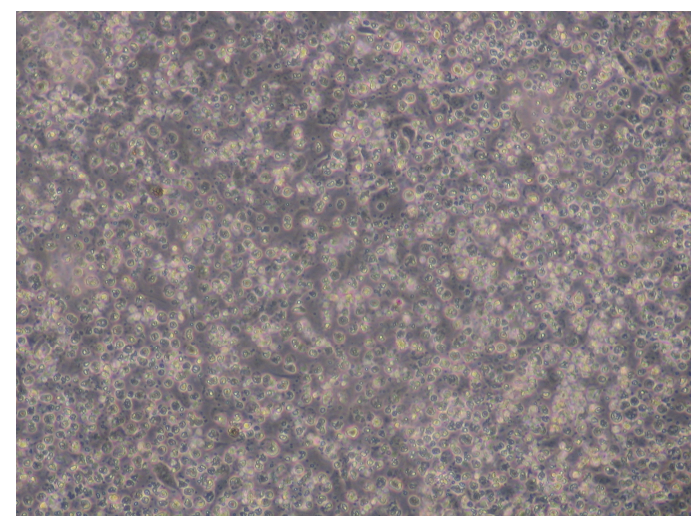

$\mathrm{t}=72$

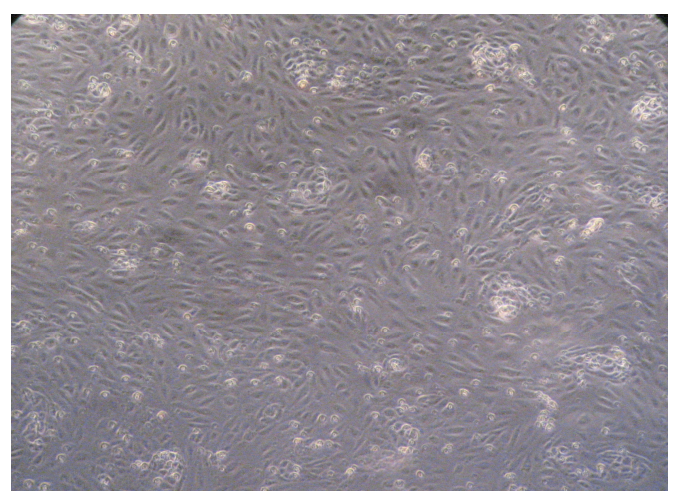

$\mathrm{t}=72$

Figure 47 - (a) The cell confluency of the HUVECs exposed to the $100 \mathrm{mg} / \mathrm{dL}$ normal glycemic cell media solution did not change from $t=0$ to $t=72$ hours. (b) Additionally, the cell confluency of the BAECs exposed to the $100 \mathrm{mg} / \mathrm{dL}$ normal glycemic cell media solution did not change from $t=0$ to $t=72$ hours either.

The evaluation of the endothelial cells under the microscope confirmed little change in cell morphology in the $100 \mathrm{mg} / \mathrm{dL}$ normal glycemic cell media solutions over the 72 hour time period.

\subsection{2 $400 \mathrm{mg} / \mathrm{dL}$ Hyperglycemic Environment}

\subsubsection{Glucose Consumption}

Figure 48 shows the recorded glucose concentrations at each time of evaluation.

Additionally, 
Table 19 lists the average glucose concentrations of each experiment, as well as the calculated difference in glucosee concentration between $\mathrm{t}=0$ and $\mathrm{t}=72$ hours.

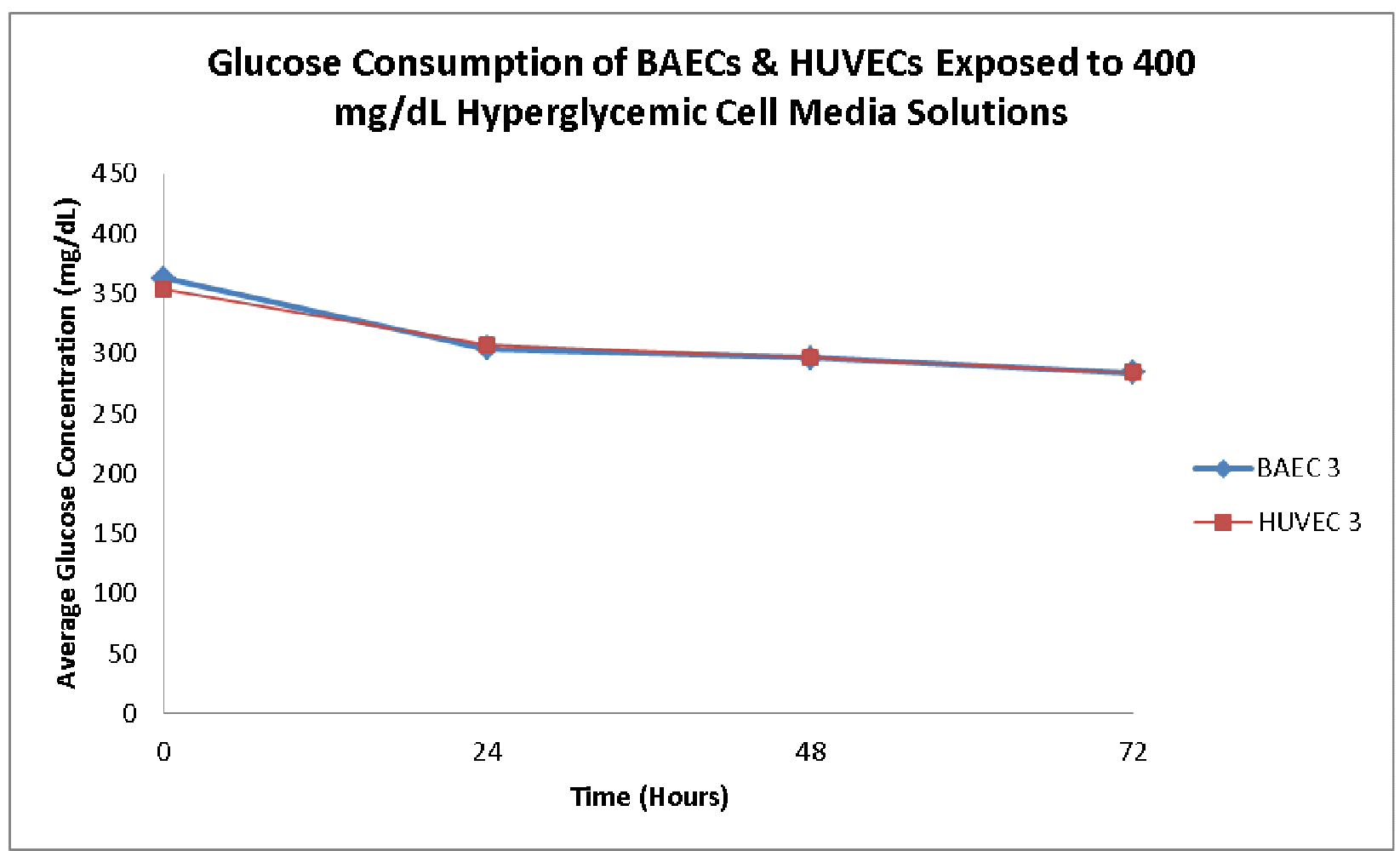

Figure 48 - The BAECs and HUVECs exposed to the $400 \mathrm{mg} / \mathrm{dL}$ hyperglycemic cell media solutions for the third experiment exhibited a decrease in glucose concentration over the 72 hour test time; thus, exhibiting glucose consumption.

Table 19 - Average Glucose Concentrations of the 400 mg/dL Hyperglycemic Cell Media Solutions Exposed to BAECs \& HUVECs of Third Experiments

\begin{tabular}{|c|c|c|c|c|}
\hline \multicolumn{4}{|c|}{$\begin{array}{c}\text { Average Glucose Concentrations of the } 400 \mathrm{mg} / \mathrm{dL} \text { Hyperglycemic } \\
\text { Cell Media Solutions Exposed to BAECs \& HUVECs of Third } \\
\text { Experiments }\end{array}$} \\
\hline \multirow{2}{*}{ Time (Hours) } & \multicolumn{4}{|c|}{$\begin{array}{c}\text { Average Glucose Concentration of Three } 400 \text { mg/dL } \\
\text { Wells }\end{array}$} \\
\cline { 2 - 5 } & BAEC 3 & HUVEC 3 & Average & St. Dev. \\
\hline 0 & 362.67 & 352.67 & 357.67 & 7.07 \\
\hline
\end{tabular}




\begin{tabular}{|c|c|c|c|c|}
\hline $\mathbf{2 4}$ & 303.67 & 306.67 & 305.17 & 2.12 \\
\hline $\mathbf{4 8}$ & 296.00 & 296.00 & 296.00 & 0.00 \\
\hline $\mathbf{7 2}$ & 283.67 & 283.67 & 283.67 & 0.00 \\
\hline$\Delta_{(\mathbf{t 0 - 7 2 )}}$ & 79.00 & 69.00 & 74.00 & 7.07 \\
\hline
\end{tabular}

The data of Figure 48 shows greater decrease in glucose concentration over the 72 hour test period. Additionally, the values recorded in

Table 19 were calculated by taking the average of the recorded glucose concentrations for all three wells containing the $400 \mathrm{mg} / \mathrm{dL}$ hyperglycemic cell media solution at each evaluation period. This table shows a significantly greater average change in glucose concentration from $t=$ 0 to $\mathrm{t}=72$ hours $-74.00 \mathrm{mg} / \mathrm{dL}$.

Again, a series of two-tailed T-tests were conducted in order to analyze whether or not there was any statistical significant difference between the average glucose concentration of the wells at $\mathrm{t}=0$ and $\mathrm{t}=72$ hours (Table 20).

Table 20 - Two-Tailed T-Tests for Final BAEC \& HUVEC Experiments Exposed to the 400 $\mathrm{mg} / \mathrm{dL}$ Hyperglycemic Cell Media Solutions

\begin{tabular}{|c|c|c|}
\hline $\begin{array}{c}\text { Two-Tailed T-Tests for Final BAEC \& HUVEC Experiments Exposed to the } 400 \\
\text { mg/dL Hyperglycemic Cell Media Solutions }\end{array}$ \\
\hline \multicolumn{3}{|c|}{$\mathrm{H}_{0}: \mu_{1}=\mu_{2} ; \mathrm{H}_{\mathrm{a}}: \mu_{1} \neq \mu_{2} ; \alpha=0.05$} \\
\hline Array 1 (hours) & Array 2 (hours) & $\boldsymbol{p}$-Value \\
\hline 0 & 24 & 0.001406033 \\
\hline
\end{tabular}




\begin{tabular}{|c|c|c|}
\hline 0 & 48 & 0.000591077 \\
\hline $\mathbf{0}$ & $\mathbf{7 2}$ & $4.39249 \mathrm{E}-05$ \\
\hline 24 & 48 & 0.452472321 \\
\hline 24 & 72 & 0.054662491 \\
\hline 48 & 72 & 0.265310104 \\
\hline
\end{tabular}

The resulting $p$-values showed that there was statistical significant difference between the average glucose concentration of the well at $\mathrm{t}=0$ and $\mathrm{t}=72$ hours.

\subsubsection{Cell Confluency}

The BAECs and HUVECs exposed to the $400 \mathrm{mg} / \mathrm{dL}$ hyperglycemic cell media solution were in a hoemstatic imbalanced environment with high glucose toxicity. Thus, the evaluation of the cells under the microscope from $t=0$ hours to $t=72$ hours should have exhibited greater decrease in cell confluncy.

Figure 49 shows that the HUVECs (Figure 49a) and the BAECs (Figure 49b) both did not exhibit significant decrease in cell conflunecy from $t=0$ hours to $t=72$ hours.

(a)

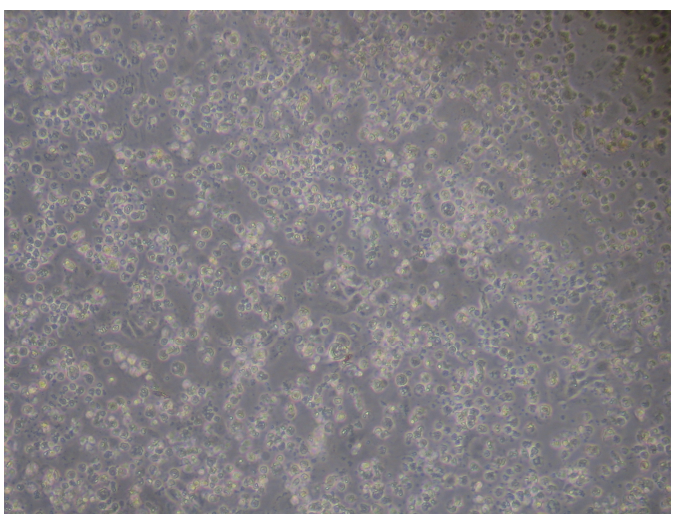

$$
\mathrm{t}=0
$$

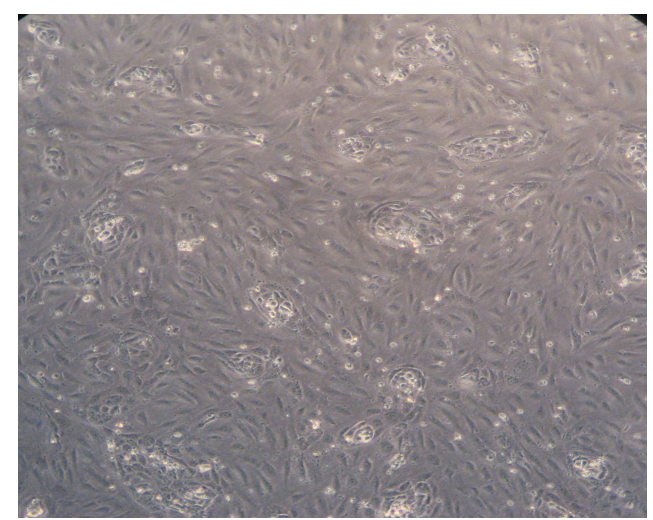

$\mathrm{t}=72$ 
(b)

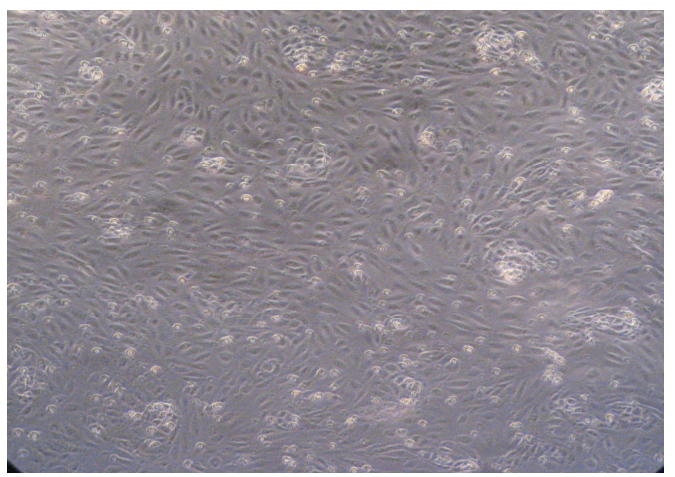

$\mathrm{t}=0$

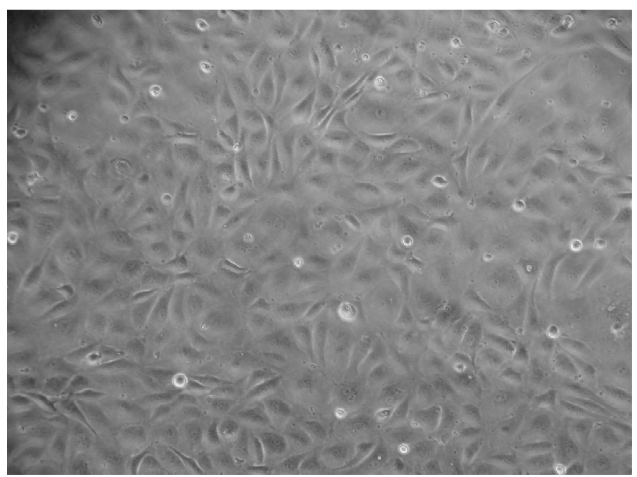

$\mathrm{t}=72$

Figure 49 - The cell confluency of the HUVECs exposed to the $400 \mathrm{mg} / \mathrm{dL}$ hyperglycemic cell media solution did not significantly decrease from $t=0$ to $t=72$ hours. (b) Additionally, the cell confluency of the BAECs exposed to the $400 \mathrm{mg} / \mathrm{dL}$ hyperglycemic cell media solution did not decrease from $t=0$ to $t=72$ hour.

The evaluation of the endothelial cells under the microscope confirmed insignificant levels of cell death in the $400 \mathrm{mg} / \mathrm{dL}$ hyperglycemic cell media solutions over the 72 hour time period.

Above all, there was still cause for concern due to the continuous low glucose concentration of the $400 \mathrm{mg} / \mathrm{dL}$ hyperglycemic cell media solution at $\mathrm{t}=0$. During this third experiment; however, it was realized that the decreased glucose concentration of the $400 \mathrm{mg} / \mathrm{dL}$ solutions in the second BAEC experiment, first two HUVEC experiments, and the third BAEC and HUVEC experiments was due to a missed step in the established procedure during the implementation of the glycemic cell media solutions to the wells containing the endothelial cells. This discovery and evaluation is discussed in Section 3.6.3 of the discussion section below.

\subsection{Discussion and Conclusion}

The purpose of the experiments in this chapter was to determine whether or not the developed $400 \mathrm{mg} / \mathrm{dL}$ hyperglycemic cell media solutions and $100 \mathrm{mg} / \mathrm{dL}$ normal glycemic cell 
media solutions would evoke a cellular response similar to cells exposed to an actual hyperglycemic environment. This was done in the attempt to confirm that a solution with an elevated glucose concentration resembles a hyperglycemic environment.

The two glycemic cell media solutions were introduced to the endothelial cells in sterile 6-well plates. The hypothesis was tested by monitoring the glucose concentration, with the Aviva Accu-Chek blood glucose meter, over a 72 hour time period at 24-hour time intervals in order to observe any glucose consumption. Additionally, the last experiment observed cell confluency of the cells under a microscope to observe any cell death.

The $400 \mathrm{mg} / \mathrm{dL}$ hyperglycemic cell media solutions were developed with the protocol established in Chapter 2. Sigma G6152 D-(+)-Glucose was added to either Lonza EBM (for experimentation with BAECs), or Gibco Medium 199 (for experimentation with HUVECs). Since the Sigma G6152 D-(+)-Glucose was an unsterile substance, the composed $400 \mathrm{mg} / \mathrm{dL}$ hyperglycemic cell media solutions had to be sterilized via syringe-filter sterilization before being introduced to the endothelial cells. The sterilization was conducted in the laminar flow hood with $10 \mathrm{~mL}$ syringes and $0.22 \mu \mathrm{m}$ syringe filters. The $100 \mathrm{mg} / \mathrm{dL}$ normal glycemic cell media solutions were already sterile; thus, did not require sterilization before application to the endothelial cells. The experiments began right when the glycemic cell media solutions were introduced to the endothelial cells and the initial evaluation at $\mathrm{t}=0$ was conducted.

\subsection{1 $100 \mathrm{mg} / \mathrm{dL}$ Normal Glycemic Environment}

According to the $p$-value calculated in Table 11, the endothelial cells exposed to the 100 $\mathrm{mg} / \mathrm{dL}$ normal glycemic cell media solutions exhibited a statistical significant difference in average glucose concentration from $t=0$ to $t=72$ hours. Although these cells were in a 
balanced glycemic environment, there was still a good amount of glucose consumption. To see a lower glucose values at $t=72$ hours compared with $t=0$ was actually not completely surprising as the cells were alive; thus, they would be consuming glucose for up to at least 72 hours. Additionally, this is why it is standard procedure in cell culture to change out the media every three days.

Although statistical analysis did show a statistical significant difference in glucose concentrations, the evaluation of the cells under the microscope showed hardly any decrease in cell confluency - meaning the $100 \mathrm{mg} / \mathrm{dL}$ normal glycemic environment did not evoke any unexpected cell death. While the initial cell morphology for the HUVECs was excellent (and remained excellent throughout the experiment), the initial cell morphology of the BAECs was poor, but was constant throughout the experiment.

\subsection{2 $400 \mathrm{mg} / \mathrm{dL}$ Hyperglycemic Environment}

The accumulated results for the initial experiments with HUVECs and BAECs indicate a greater amount of glucose consumption in the $400 \mathrm{mg} / \mathrm{dL}$ hyperglycemic environment. Additionally, the average $\Delta_{(\mathrm{t} 0-\mathrm{t} 72)}$ of the glucose concentration in the cells was $38.75 \mathrm{mg} / \mathrm{dL}$, which is greater than the glucose consumption of the cells in the $100 \mathrm{mg} / \mathrm{dL}$ normal glycemic cell media solutions that only had an average $\Delta_{(\mathrm{t} 0-\mathrm{t} 72)}$ of $11.58 \mathrm{mg} / \mathrm{dL}$. However, although there was a high number of consumption when comparing the two environments, relative to one another, the consumption was not drastically greater in the $400 \mathrm{mg} / \mathrm{dL}$ wells than the $100 \mathrm{mg} / \mathrm{dL}$ wells (12.14\% vs. $12.64 \%$ for BAEC \& HUVEC experiments $1 \& 2)$. The average change in glucose concentration would most likely have been even greater for the $400 \mathrm{mg} / \mathrm{dL}$ environment 
had the error not occurred with the three experiments that had a substantially lower glucose concentration than planned at $\mathrm{t}=0$ hours.

However, statistical analysis confirmed no statistical significant difference in the average glucose concentrations between any of the evaluation periods. Obviously, the analysis was biased due to the unexpected low glucose concentrations recorded at $\mathrm{t}=0$ hours for the second BAEC experiment and the first two HUVEC experiments. Additionally, the analyzed sample size was small; therefore, the calculated $p$-values may not have given a fair evaluation of the endothelial cells exposed to the $400 \mathrm{mg} / \mathrm{dL}$ hyperglycemic cell media solutions.

Since the first BAEC experiment ran as expected, the same two-tailed T-tests were conducted on only the data accumulated from the first BAEC experiment. Table 21 shows the calculated $p$-values.

Table 21 - Two-Tailed T-Test Results for First BAEC Experiment Exposed to $400 \mathrm{mg} / \mathrm{dL}$ Hyperglycemic Cell Media Solutions

\begin{tabular}{|c|c|c|}
\hline \multicolumn{3}{|c|}{$\begin{array}{l}\text { Two-Tailed T-Test Results for the First BAEC Experiment Exposed to the } 4 \\
\text { mg/dL Hyperglycemic Cell Media Solutions }\end{array}$} \\
\hline \multicolumn{3}{|c|}{$H_{0}: \mu_{1}=\mu_{2} ; H_{a}: \mu_{1}=\mu_{2} ; \alpha=0.05$} \\
\hline Array 1 (hours) & Array 2 (hours) & $p$-Value \\
\hline 0 & 24 & 0.004460281 \\
\hline 0 & 48 & 0.003424838 \\
\hline $\mathbf{0}$ & 72 & 0.001345804 \\
\hline 24 & 48 & 0.979821034 \\
\hline 24 & 72 & 0.423717128 \\
\hline 48 & 72 & 0.413348961 \\
\hline
\end{tabular}


The results of these t-tests confirmed a statistical significant difference between the average glucose concentrations at $\mathrm{t}=0$ and $\mathrm{t}=72$ hours. In addition, the calculated $p$-value was less than the calculated $p$-value comparing the average glucose concentrations at $t=0$ and 72 hours for the $100 \mathrm{mg} / \mathrm{dL}$ wells; therefore, supporting the test's original hypothesis that the endothelial cells exposed to the $400 \mathrm{mg} / \mathrm{dL}$ hyperglycemic cell media solutions would experience greater glucose consumption than the endothelial cells exposed to the $100 \mathrm{mg} / \mathrm{dL}$ cell media. Though this was the case for the first BAEC experiment, recall, the same outcome did not occur for all experiments.

The final BAEC and HUVEC experiments accumulated increased results with an average $\Delta_{\text {(t0-t72) }}$ of $74.00 \mathrm{mg} / \mathrm{dL}$. Thus, greater glucose consumption was observed in the third experiment by the BAECs and HUVECs exposed to the $400 \mathrm{mg} / \mathrm{dL}$ hyperglycemic cell media solutions compared to the cells exposed to the $100 \mathrm{mg} / \mathrm{dL}$ normal glycemic cell media solutions, which had an average $\Delta_{(\mathrm{t} 0-\mathrm{t} 72)}$ of $9.00 \mathrm{mg} / \mathrm{dL}$. Also, the $\Delta_{(\mathrm{t} 0-\mathrm{t} 72)}$ observed in the $400 \mathrm{mg} / \mathrm{dL}$ wells greater than the $\Delta_{(\mathrm{t} 0-\mathrm{t} 72)}$ of the initial BAEC \& HUVEC experiments. Additionally, the calculated $p$-value was smaller than the $p$-value recorded comparing the average glucose concentration of the wells exposed to the $100 \mathrm{mg} / \mathrm{dL}$ normal glycemic cell media solution between $\mathrm{t}=0$ hours and $\mathrm{t}=72$ hours. The greater statistical difference confirms that there was greater glucose consumption by the endothelial cells exposed to the hyperglycemic environment in these particular experiments.

There was little evidence of any cell death of the BAECs and HUVECs exposed to the $400 \mathrm{mg} / \mathrm{dL}$ hyperglycemic cell media solutions. At first this was discouraging; however, the unsubstantial amount of cell death in the $400 \mathrm{mg} / \mathrm{dL}$ environment is a preferable outcome as this 
hyperglycemic environment can now be an adequate environment to evaluate endothelial cells in a hyperglycemic BVM.

However, even though additional glucose was added to the original recipe for the 400 $\mathrm{mg} / \mathrm{dL}$ hyperglycemic solution, to compensate for any lost glucose during the sterilization, the results still showed slightly low glucose concentrations at $\mathrm{t}=0-$ roughly $357.67 \mathrm{mg} / \mathrm{dL}$. Thus, something other than the sterilization process was diluting the hyperglycemic solution. The following section discusses the resolution.

\subsubsection{Removal Complete Cell Media during Application of Glycemic Cell Media Solutions}

The initial part of the experiment required the BAECs and HUVECs to be cultured into 6-well plates and routinely fed with approximately $2-3 \mathrm{~mL}$ of complete cell media (base media plus all the aliquots). This media was also a "low glucose" cell media like the Gibco Medium 199 and Lonza EBM; thus, it already contained $100 \mathrm{mg} / \mathrm{dL}$ of glucose. In addition, the procedure for the first BAEC experiment was conducted by a trained lab technician; therefore, there was no error in the procedure and the results of this experiment came out as expected. Unfortunately, one significant step in the procedure was missed when observing and developing the protocol. Before introducing the glycemic cell media to the endothelial cells, it was necessary to remove the complete cell media feeding the cultured endothelial cells first. It seems like an obvious step in the protocol; however, throughout the strict maintenance of a sterile environment during these experiments, it can be an obvious but easy step to miss. 
Recall, approximately $3 \mathrm{~mL}$ of glycemic cell media solution was added to each well during the second BAEC experiment and the first two HUVEC experiments. This volume of was increased to $6 \mathrm{~mL}$ for the third BAEC and HUVEC experiments. Assuming that the wells originally contained $2 \mathrm{~mL}$ of the homogenous cell media, the amount of glucose in the small volume of added glycemic cell media solution $(3 \mathrm{~mL}$ or $6 \mathrm{~mL}$ ) was calculated using the amount of glucose weighed and added to create the solutions, and then adjusted to compensate for the additional $2 \mathrm{~mL}$ of complete "low glucose" cell media. Table 22 shows the results of these calculations.

Table 22 - Calculated Glucose Concentrations Compensating for Additional $2 \mathrm{~mL}$ of Complete Cell Media that was mistakenly not removed

\begin{tabular}{|c|c|c|c|c|c|c|}
\hline Experiment & $\begin{array}{c}\text { Glucose } \\
\text { Added (g) }\end{array}$ & $\begin{array}{l}\text { Cell Media } \\
\text { Volume } \\
\text { (mL) }\end{array}$ & $\begin{array}{l}\text { Assumed } \\
\text { Volume of } \\
\text { Complete } \\
\text { "Low } \\
\text { Glucose" Cell } \\
\text { Media (mL) }\end{array}$ & $\begin{array}{l}\text { Volume of } \\
\text { Applied } \\
\text { Glycemic } \\
\text { Cell Media } \\
\text { Solution } \\
\text { (mL) }\end{array}$ & $\begin{array}{l}\text { Average } \\
\text { Glucose } \\
\text { Concentration } \\
\text { of Wells } \\
(\mathrm{mg} / \mathrm{dL})\end{array}$ & $\begin{array}{c}\text { Adjusted } \\
\text { Glucose } \\
\text { Concentration } \\
\text { of Wells } \\
\text { (mg/dL) }\end{array}$ \\
\hline BAEC 2 & 0.0613 & 20 & 2 & 3 & 287.67 & 223.90 \\
\hline $\begin{array}{l}\text { HUVEC } 1 \\
\text { HUVEC } 2\end{array}$ & 0.1214 & 40 & 2 & 3 & 246.67 & 222.10 \\
\hline BAEC 3 & 0.0813 & 20 & 2 & 6 & 362.67 & 328.75 \\
\hline
\end{tabular}




\begin{tabular}{|l|l|l|l|l|l|l|}
\hline HUVEC 3 & 0.0807 & 20 & 2 & 6 & 352.67 & 327.63 \\
\hline
\end{tabular}

For example, the adjusted glucose concentration of the BAECs from the second experiment was calculated as follows:

1. Find the amount of glucose in the volume of applied glycemic cell media solution.

$$
\begin{aligned}
& 0.0613 \mathrm{~g} / 20 \mathrm{~mL}=\mathrm{x} \mathrm{g} / 3 \mathrm{~mL} \\
& \mathrm{x}=0.009195 \mathrm{~g} / 3 \mathrm{~mL}
\end{aligned}
$$

2. Compensate the result by adding the addition $2 \mathrm{~mL}$ of "low glucose" media.

$$
0.009195 \mathrm{~g} / 3 \mathrm{~mL}+0.002 \mathrm{~g} / 2 \mathrm{~mL}=0.011195 \mathrm{~g} / 5 \mathrm{~mL}
$$

3. Convert $\mathrm{g} / \mathrm{mL}$ to $\mathrm{mg} / \mathrm{dL}$.

$$
0.011195 \mathrm{~g} / 5 \mathrm{~mL} \times 1000 \mathrm{mg} / 1 \mathrm{~g} \times 100 \mathrm{~mL} / 1 \mathrm{dL}=223.90 \mathrm{mg} / \mathrm{dL}
$$

The calculated adjustments are slightly smaller than the recorded average glucose concentrations; however, this calculation was conducted assuming $2 \mathrm{~mL}$ of homogenous media was already in the wells. Nonetheless, the adjustments confirmed that this incorrect step in the protocol was the most likely root cause for the decreased glucose concentration of the $400 \mathrm{mg} / \mathrm{dL}$ hyperglycemic cell media solutions at the $t=0$ evaluation time. In the future, similar studies should be contucted by removing all normoglycemic complete media before adding the hyperglycemic media, so that the composition of media exposed to the cells is well controlled. 


\subsubsection{Summary}

A total of six studies were conducted for this chapter - three studies with BAECs and three studies with HUVECs. There were four key factors that differed among the experiments:

1. Adding Glycemic Cell Media Solutions

a. $10 \mathrm{~mL}$ of the glycemic cell media solution was added to each well for the first BAEC experiment.

b. $3 \mathrm{~mL}$ of glycemic cell media solution was added to each well for the second BAEC experiment and first two HUVEC experiments.

c. $6 \mathrm{~mL}$ of glycemic cell media solution was added each well for the third BAEC and HUVEC experiments.

2. The first and second HUVEC experiments used the same $400 \mathrm{mg} / \mathrm{dL}$ hyperglycemic cell media solution.

3. The third BAEC and HUVEC experiments had additional glucose added to compensate for any lost glucose during the sterilization process.

4. Cell morphology was evaluated and recorded for only the third BAEC and HUVEC experiments.

In addition, Table 23 summarizes the conclusions derived from the results of the experiments of this chapter.

Table 23 - Summary of Conclusions from Experimentation with BAECs \& HUVECs in 100 $\mathrm{mg} / \mathrm{dL} \& 400 \mathrm{mg} / \mathrm{dL}$ Environments 


\begin{tabular}{|c|c|c|c|c|c|c|}
\hline \multirow{2}{*}{$\begin{array}{c}\text { Glycemic } \\
\text { Environment }\end{array}$} & \multicolumn{2}{|c|}{ Hypothesis } & \multicolumn{2}{|c|}{ Results } & \multicolumn{2}{|c|}{ Conclusion } \\
\hline & $\begin{array}{c}\text { Glucose } \\
\text { Consumption }\end{array}$ & $\begin{array}{c}\text { Cell } \\
\text { Morphology }\end{array}$ & $\begin{array}{c}\text { Glucose } \\
\text { Consumption }\end{array}$ & $\begin{array}{c}\text { Cell } \\
\text { Confluency }\end{array}$ & $\begin{array}{c}\text { Glucose } \\
\text { Consumption }\end{array}$ & $\begin{array}{c}\text { Cell } \\
\text { Confluency }\end{array}$ \\
\hline $\begin{array}{c}100 \mathrm{mg} / \mathrm{dL} \\
\text { Normal } \\
\text { Glycemic Cell } \\
\text { Media } \\
\text { Solutions }\end{array}$ & $\begin{array}{l}\text { Minimal } \\
\text { decrease in } \\
\text { glucose } \\
\text { concentration } \\
\text { = minimal } \\
\text { glucose } \\
\text { consumption }\end{array}$ & $\begin{array}{c}\text { Minimal } \\
\text { decrease in } \\
\text { cell } \\
\text { confluency = } \\
\text { minimal cell } \\
\text { death }\end{array}$ & $\begin{array}{l}\text { Statistically } \\
\text { significant } \\
\text { difference in } \\
\text { average } \\
\text { glucose } \\
\text { concentration } \\
\text { between } \mathrm{t}=0 \\
\& \mathrm{t}=72 \\
\text { hours }\end{array}$ & $\begin{array}{l}\text { Little or no } \\
\text { decrease in } \\
\text { cell } \\
\text { confluency }\end{array}$ & $\begin{array}{c}\text { Cells are } \\
\text { active and } \\
\text { consuming } \\
\text { glucose; } \\
\text { however, not } \\
\text { as much as } \\
\text { cells exposed } \\
\text { to } \\
\text { hyperglycemic } \\
\text { environment. }\end{array}$ & $\begin{array}{c}\text { Cells are } \\
\text { active and } \\
\text { healthy in } 100 \\
\mathrm{mg} / \mathrm{dL} \text { normal } \\
\text { glycemic } \\
\text { environment. } \\
\text { Cell death is } \\
\text { not evoked. }\end{array}$ \\
\hline $\begin{array}{c}400 \mathrm{mg} / \mathrm{dL} \\
\text { Hyperglycemic } \\
\text { Cell Media } \\
\text { Solutions }\end{array}$ & $\begin{array}{l}\text { Significant } \\
\text { decrease in } \\
\text { glucose } \\
\text { concentration } \\
=\text { significant } \\
\text { glucose } \\
\text { consumption }\end{array}$ & $\begin{array}{c}\text { Significant } \\
\text { decrease in } \\
\text { cell } \\
\text { confluency }= \\
\text { significant } \\
\text { cell death }\end{array}$ & $\begin{array}{c}\text { Statistically } \\
\text { significant } \\
\text { difference in } \\
\text { average } \\
\text { glucose } \\
\text { concentration } \\
\text { between } \mathrm{t}=0 \\
\& \mathrm{t}=72 \\
\text { hours }\end{array}$ & $\begin{array}{c}\text { Little } \\
\text { decrease in } \\
\text { cell } \\
\text { confluency }\end{array}$ & $\begin{array}{l}\text { Cells are more } \\
\text { active and } \\
\text { consuming } \\
\text { higher } \\
\text { amounts of } \\
\text { glucose } \\
\text { compared to } \\
\text { normal } \\
\text { glycemic } \\
\text { environment. }\end{array}$ & $\begin{array}{c}\text { Cells are able } \\
\text { to survive } 400 \\
\text { mg/dL } \\
\text { hyperglycemic } \\
\text { environment. } \\
\text { Allows for } \\
\text { adequate } \\
\text { evaluation of } \\
\text { cells within } \\
\text { hyperglycemic } \\
\text { BVM. }\end{array}$ \\
\hline
\end{tabular}

Thus, pardoning the improper step conducted during the procedure of the experiments, in conclusion, the results of the BAEC and HUVEC experiments did in fact support the hypothesis that the developed $400 \mathrm{mg} / \mathrm{dL}$ hyperglycemic cell media evoked the expected glucose consumption, although cell morphology was not drastically affected. Although the results did not accumulate as planned, the constant inquiries contributed to an excellent learning experience.

Finally, the high glucose concentrated solutions developed in Chapter 2 evoked one of the expected characteristics that endothelial cells would exhibit in an actual hyperglycemic environment: they increased glucose consumption. Though this was not true for all the conducted experiments, the results did show that hyperglycemic solutions can be introduced to endothelial cells, the glucose concentration can be monitored over time (no matter what the initial concentration is), and endothelial cells are able to survive high glycemic environments, which would allow for adequate evaluation of BVMs introduced to various glycemic environments. The conclusions from this chapter open the opportunity to further evaluate the 
effects induced by various glycemic environments by analyzing the phenotype and expression of endothelial cells exposed to these environments.

\section{Chapter 4: Discussion \& Conclusion}




\subsection{Overview of Thesis}

\subsubsection{Diabetes Mellitus}

Diabetes Mellitus is a disease that affects the body's ability to properly produce and use insulin in order to metabolize blood glucose and maintain an adequate blood glucose level. At first, the disease was a slow and painful death. While years of research were conducted on attempting to cure the disease, the first breakthrough didn't occur until 1879 when Joseph Von Mering and Oscar Minkowski, of Germany, discovered that removing the pancreas of a dog caused the dog to develop diabetes as the dog excessively urinated and the urine contained high levels of glucose [1]. Thus, the anti-diabetic substance researchers were looking for was within the pancreas. While many tried to extract a purified sample of the substance for human use, it was an extremely challenging procedure - to the point where many scientists were giving up and losing hope. However, in 1921, Dr. Fredrick Banting, Dr. James M Macleod, and James B. Collip, from the University of Toronto, were able to obtain the first purified sample for human testing - insulin [3]. Insulin drastically improved the condition of diagnosed patients, and the discovery awarded Banting and Macleod the Nobel Prize in Medicine in 1923 [3]. Due to this discovery, what used to be a horrifying experience for physicians and patients became an open door for new research in quest of a cure for the disease.

There are two common types of diabetes mellitus - type 1 diabetes mellitus, and type II diabetes mellitus. Type I diabetes is an autoimmune that involves the degeneration of pancreatic $\beta$-cells - cells that produce and secrete insulin - thus, patients are unable to regulate blood glucose levels and require constant blood glucose monitoring and injections of insulin. Type II diabetes mellitus is different from Type $\mathrm{I}$ in in that that the pancreatic $\beta$-cells are impaired and 
cannot produce proper amount of insulin, and the body cells exhibit a resistance to the insulin. Thus, patients are unable to regulate blood glucose levels with only insulin injections and require an overall lifestyle adjustment with an improved diet and increased level of exercise, along with medications.

\subsubsection{Complications of Diabetes Mellitus}

Diabetes mellitus involves homeostatic imbalance in the body's blood sugar; therefore, hyperglycemia is a chronic condition associated with diabetes - especially type II diabetics. Chronic hyperglycemia can lead to significant complications, such as hypertension, ketoacidosis, nephropathy, neuropathy, and cardiovascular disease - particularly, coronary artery disease (CAD) [88]. Since patients with type I diabetes are able to manage their blood glucose levels well with continuous insulin injections, most complications are frequently seen in patients with type II diabetes. Out of all these major complications, this thesis focused on the condition of coronary artery disease, as it is the significant contributing factor to the high mortality rate seen with type II diabetics.

Coronary artery disease results in the narrowing of the coronary arteries due to atherosclerosis - the build-up of plaques within the arteries. The hyperglycemic environment in diabetics contributes to an increase of endothelial cell dysfunction in coronary blood vessels, making diabetics more susceptible to atherosclerotic build-up. Revascularization treatments are necessary for patients with $\mathrm{CAD}$, and the most common treatment is coronary stent implantation [69]. Numerous studies show that CAD patients with diabetes experience a greater need for coronary stent replacement procedures and have a lower overall survival rate following the coronary therapy procedure compared to non-diabetic patients [153]. CAD patients with 
diabetes especially experience a high rate of restenosis and a need for repeat revascularization with coronary stent replacement. In-stent restenosis is the reformation of atherosclerotic blockage due to the vascular injury caused by the implantation of the stent. Studies show that hyperglycemia is correlated with high levels of expression of vascular adhesion molecules throughout coronary blood vessels, leading to the high rate of in-stent restenosis [154]. Even with the introduction of drug-eluting stents, the rate of restenosis is still high among the CAD population with diabetes.

Due to this high failure rate of stents in diabetics, it would be beneficial to have a testing system that could be used to optimize stent configurations and coatings for diabetic patients. While in vivo animal models and clinical testing are vital for product evaluation, it would also be valuable to test and evaluate stent designs prior to in vivo environments with an in vitro test that resembles the physiologic environment of a native diabetic blood vessel - a diabetic blood vessel $\operatorname{mimic}(\mathrm{BVM})$ [155].

\subsubsection{Purpose \& Direction of Study}

The purpose of this thesis was to demonstrate the concept that a diabetic environment could be replicated by inducing a high glucose concentrated environment in the attempt to resemble a hyperglycemic environment. This thesis was driven by the notion that a high concentration of glucose would properly replicate the chronic state of hyperglycemia seen in a diabetic environment. Although the scope of this concept was beyond a single thesis, the goal of the present work was to take initial steps towards creating a hyperglycemic in vitro environment. In order to achieve this goal, the thesis was broken down into three major parts:

1. Establish a protocol to create high glucose solutions in standard cell culture media 
2. Establish a protocol to accurately monitor the concentrations of the high glucose solutions

3. Test this monitoring protocol in cell culture over time and determine how the high glucose solutions affect endothelial cells

This was done to serve the single purpose of determining whether or not the hyperglycemic solutions could be induced into an in vitro BVM.

\subsubsection{Method of Creating \& Monitoring Glycemic Cell Media Solutions}

The first stage of the thesis was to establish a protocol of creating glycemic cell media solutions at specific glucose concentrations. Preliminary tests were conducted and a feasible method was established using Sigma G6152 D-(+)-Glucose and Gibco Medium 199.

The next stage was to test and establish a method to accurately measure the glucose concentration of the glycemic solutions. After studying the literature, the most feasible methods that were tested were the blood glucose meter (specifically the Aviva Accu-Chek blood glucose meter) and the Glucoflex-R visual blood glucose test strips. The goal of these experiments was to determine whether or not the Aviva Accu-Chek blood glucose meter and Glucoflex-R visual blood glucose test strips could accurately and precisely record the glucose concentrations of developed $200 \mathrm{mg} / \mathrm{dL}, 350 \mathrm{mg} / \mathrm{dL}$ and $500 \mathrm{mg} / \mathrm{dL}$ glycemic cell media solutions. The results confirmed two things:

1. The Aviva Accu-Chek blood glucose meter was the best method of monitoring the developed glycemic solutions as it was the most accurate and precise method. 
2. The base Gibco Medium 199 was a "low glucose" solution that already contained $100 \mathrm{mg} / \mathrm{dL}$ of D-glucose.

\subsubsection{Monitoring Glucose Consumption of BAECs and HUVECs}

Once the method of creating and monitoring glycemic cell media solutions was established, the next stage was to assess whether the developed glycemic cell media solutions affected bovine aortic endothelial cells (BAECs) and human umbilical vein endothelial cells (HUVECs) in culture. Base cell medium was used as the control at $100 \mathrm{mg} / \mathrm{dL}$, and was compared to a developed $400 \mathrm{mg} / \mathrm{dL}$ hyperglycemic cell media solution. Glucose consumption was evaluated by monitoring the glucose concentration of the glycemic cell media solutions, and the cell death was evaluated, for only the third set of experiments, by monitoring the cell confluency of each well under a microscope.

The endothelial cells exposed to the $100 \mathrm{mg} / \mathrm{dL}$ normal glycemic cell media solution exhibited a statistically significant amount of glucose consumption. Additionally, there was little or no cell death observed in the $100 \mathrm{mg} / \mathrm{dL}$ wells for the final experiments. The endothelial cells exposed to the $400 \mathrm{mg} / \mathrm{dL}$ hyperglycemic cell media solution exhibited greater glucose consumption only during the final experiments; however, these cells did not exhibit significant cell death throughout the experiment. The results of the initial experiments did not exhibit the expected glucose consumption of the endothelial cells within the $400 \mathrm{mg} / \mathrm{dL}$ environment, and further investigation uncovered the cause of the obscure results. An important step in the protocol of these experiments was to first remove the complete cell media before adding the glycemic cell media. By not conducting this step, the glycemic solutions were diluted and resulted in low glucose concentrations at $\mathrm{t}=0$. 
Therefore, the results of the initial test showed no difference in glucose consumption between the cells exposed to the $100 \mathrm{mg} / \mathrm{dL}$ environment and the cells exposed to the $400 \mathrm{mg} / \mathrm{dL}$ environment. However, the results of the final experiments did exhibit greater glucose by the endothelial cells, especially in comparison to the initial experiments. Additionally, the endothelial cells in the $400 \mathrm{mg} / \mathrm{dL}$ environment did not exhibit significant cell death from $\mathrm{t}=0$ to $t=72$ hours. These results show that the composed glycemic solutions can be introduced to endothelial cells, the glucose concentration of the glycemic solutions can be monitored over time (no matter what the initial concentration) with an Aviva Accu-Chek blood glucose meter, and the endothelial cells are able to survive a high glucose concentrated solution up to $400 \mathrm{mg} / \mathrm{dL}$. All of these steps lead towards to development of a replicable hyperglycemic environment within a BVM.

Table 24 summarizes the experiments, results, and conclusions derived from this entire thesis.

Table 24 - Summary of Experimentation, Results, and Conclusions of Entire Thesis

\begin{tabular}{|c|c|c|c|}
\hline \multicolumn{3}{|c|}{ Summary of Conclusions from Experimentation of Thesis } \\
\hline Test & Hypothesis & Results & Conclusions \\
\hline $\begin{array}{c}\text { Development of Glycemic } \\
\text { Solution Recipes }\end{array}$ & $\begin{array}{c}\text { Adding } x \text { g of Sigma } \\
\text { G6152 D-(+)-Glucose to } y\end{array}$ & $\begin{array}{c}\text { Initial readings were high } \\
\text { by } 100 \mathrm{mg} / \mathrm{dL} \text {. Aviva }\end{array}$ & $\begin{array}{c}\text { 1. Base cell media is a } \\
\text { "low glucose" }\end{array}$ \\
\hline
\end{tabular}




\begin{tabular}{|c|c|c|c|}
\hline & $\begin{array}{c}\mathrm{mL} \text { of base cell media will } \\
\text { yield a solution with a } \\
\text { glucose concentration of } z \\
\mathrm{mg} / \mathrm{dL}\end{array}$ & \multirow{2}{*}{$\begin{array}{l}\text { Accu-Chek blood glucose } \\
\text { meter had precise } \\
\text { measurements of all } \\
\text { glucose concentration. } \\
\text { The Glucoflex-R visual } \\
\text { blood glucose strips } \\
\text { recorded imprecise results } \\
\text { with extremely high } \\
\text { variability. }\end{array}$} & \multirow{2}{*}{$\begin{array}{l}\text { solution that contains } \\
100 \mathrm{mg} / \mathrm{dL} \text { of D- } \\
\text { glucose } \\
\text { 2. Established protocol } \\
\text { to develop various } \\
\text { glycemic solutions is } \\
\text { a valid method. } \\
\text { 3. Aviva Accu-Chek } \\
\text { blood glucose meter } \\
\text { accurately and } \\
\text { precisely measured } \\
\text { glucose concentration } \\
\text { of developed } \\
\text { glycemic cell media } \\
\text { solutions }\end{array}$} \\
\hline $\begin{array}{c}\text { Method of Monitoring } \\
\text { Glucose Concentration of } \\
100 \mathrm{mg} / \mathrm{dL}, 250 \mathrm{mg} / \mathrm{dL} \text {, } \\
\text { and } 400 \mathrm{mg} / \mathrm{dL}\end{array}$ & $\begin{array}{l}\text { The Aviva Accu-Chek } \\
\text { blood glucose meter and } \\
\text { Glucoflex-R visual blood } \\
\text { glucose strips can } \\
\text { accurately and precisely } \\
\text { measure the glucose } \\
\text { concentration of various } \\
\text { glycemic solutions } \\
\text { developed using the } \\
\text { established protocol }\end{array}$ & & \\
\hline $\begin{array}{l}\text { Evaluation of Endothelial } \\
\text { Cells in } 100 \mathrm{mg} / \mathrm{dL} \text { and } \\
400 \mathrm{mg} / \mathrm{dL} \text { Environments }\end{array}$ & $\begin{array}{l}\text { BAECs and HUVECs } \\
\text { exposed to } 400 \mathrm{mg} / \mathrm{dL} \\
\text { hyperglycemic cell media } \\
\text { solution will exhibit } \\
\text { greater glucose } \\
\text { consumption and cell } \\
\text { death than ECs exposed to } \\
100 \mathrm{mg} / \mathrm{dL} \text { normal } \\
\text { glycemic cell media } \\
\text { solution }\end{array}$ & $\begin{array}{l}\text { 1. Initial experiments } \\
\text { showed statistically } \\
\text { significant decrease in } \\
\text { glucose concentration } \\
\text { in } 100 \mathrm{mg} / \mathrm{dL} \text { solutions, } \\
\text { but insignificant } \\
\text { decrease in glucose } \\
\text { concentration in } 400 \\
\text { mg/dL solutions. } \\
\text { Additionally, all } 400 \\
\text { mg/dL environments } \\
\text { (except for BAEC } 1) \\
\text { had low glucose } \\
\text { concentrations at } \mathrm{t}=0 . \\
\text { 2. Final experiments } \\
\text { showed significant } \\
\text { decrease in glucose } \\
\text { concentration in } 100 \\
\text { mg/dL solutions, and } \\
\text { even greater decrease } \\
\text { in glucose } \\
\text { concentration in } 400 \\
\text { mg/dL solutions. } \\
\text { 3. Final experiments } \\
\text { showed no significant } \\
\text { decrease in cell } \\
\text { confluency for neither } \\
\text { the cells in the } 100 \\
\text { mg/dL nor the } 400 \\
\text { mg/dL environments. }\end{array}$ & $\begin{array}{l}\text { 1. Unexpected decrease } \\
\text { in glucose } \\
\text { concentration for } \\
\text { initial experiments } \\
\text { was caused by not } \\
\text { removing "complete" } \\
\text { cell media already in } \\
\text { the wells. This } \\
\text { diluted the } 400 \mathrm{mg} / \mathrm{dL} \\
\text { solutions. } \\
\text { 2. Cells in both the } 100 \\
\text { mg/dL and } 400 \\
\text { mg/dL solutions } \\
\text { exhibited glucose } \\
\text { consumption; } \\
\text { however, final } \\
\text { experiments showed } \\
400 \mathrm{mg} / \mathrm{dL} \\
\text { environment to } \\
\text { promote greater } \\
\text { consumption. } \\
\text { 3. } 400 \mathrm{mg} / \mathrm{dL} \\
\text { environment did not } \\
\text { evoke greater cell } \\
\text { death than the } 100 \\
\text { mg/dL environment. } \\
\text { This is acceptable as } \\
\text { now } 400 \mathrm{mg} / \mathrm{dL} \\
\text { environment may be } \\
\text { used to evaluate } \\
\text { endothelial cells } \\
\text { within a diabetic } \\
\text { BVM. }\end{array}$ \\
\hline
\end{tabular}




\subsection{Discussion of Thesis}

\subsubsection{Improvements to Test Protocols}

The experiments conducted during this thesis made initial steps towards creating an environment that can resemble a hyperglycemic environment to potentially develop a diabetic blood vessel mimic for stent evaluation. With the completion of this thesis, a protocol is established for the development of hyperglycemic cell media solutions for incorporation with BVMs. In addition, the glucose concentration of these solutions can be accurately and precisely measured with an Accu-Chek blood glucose meter.

As with any set of experiments, there were steps in the procedure that caused trouble and likely caused obscurities in the data. For example, one significant observation was the inaccuracy of the balance used to weigh the initial amount of Sigma G6512 D-(+)-glucose. Although the balance read the desired mass, the reading frequently decreased as much as 0.02 $\mathrm{mg}$ in one minute. Although this seems to be an insignificant amount, remember that an extremely small amount of glucose can alter the glucose concentration of a glycemic cell media solution drastically. Thus, it wasn't uncommon for multiple measurements to ensure that the correct amount of glucose was used for a particular glycemic cell media solution.

The potential for error when measuring the mass of glucose was a continuous problem for the accuracy of the experiments. A small volume of cell media was used for many of the experiments (20-40 mL); thus, the amount of glucose added to each solution was extremely small as well. Therefore, the established protocol, for the development of the glycemic cell media solutions, calls for Sigma G6152 D-(+)-Glucose to be added to at least $100 \mathrm{~mL}$ of cell 
media. For example, in order to make $20 \mathrm{~mL}$ of $400 \mathrm{mg} / \mathrm{dL}$ hyperglycemic cell media solution, approximately $0.060 \mathrm{~g}$ of glucose was added to $20 \mathrm{~mL}$ of cell media solution (this was done via calculations and compensation of the $100 \mathrm{mg} / \mathrm{dL}$ of glucose already in the base cell media).. In order to make $20 \mathrm{~mL}$ of $200 \mathrm{mg} / \mathrm{dL}$ hyperglycemic cell media solution, approximately $0.030 \mathrm{~g}$ of glucose needs to be added to the $20 \mathrm{~mL}$ of cell media. Thus, only a difference of $0.030 \mathrm{~g}$ of glucose differentiates $200 \mathrm{mg} / \mathrm{dL}$ from $400 \mathrm{mg} / \mathrm{dL}$. However, if the volume of media was increased to as much as $1000 \mathrm{~mL}$, then a $400 \mathrm{mg} / \mathrm{dL}$ hyperglycemic solution would require approximately $3.0 \mathrm{~g}$ of glucose, while a $250 \mathrm{mg} / \mathrm{dL}$ hyperglycemic solution would require approximately $1.5 \mathrm{~g}$ of glucose - a difference of $1.5 \mathrm{~g}$ is a much more practical margin of error than a difference of $0.03 \mathrm{~g}$.

Another error that occurred was the use of the incorrect media during test with the BAECs and HUVECs. For the initial measurements (tests conducted for Chapter 2), the Gibco Medium 199 was the correct media to use. However, the normal complete BAEC media and the normal complete HUVEC media should have been used for the experiments of Chapter 3. This is BAEC \& HUVEC base media with all the necessary aliquots. Using the Gibco Medium 199 for the HUVECs and the Lonza EBM did not hamper the experiments, but it should be noted that any further experiments should be conducted using the cell type's normal, complete media.

Lastly, using the Aviva Accu-Chek blood glucose meter was a practical and extremely convenient method of measuring the glucose concentration of glycemic cell media solutions. However, it was crucial to maintain the sterility of the test strips. The strips are sterile; thus, must be handled in a sterile environment only to avoid any contamination - especially when used with the endothelial cells. One consistent problem was remembering to not expose the strips outside of the laminar hood, which was necessary since many glucose concentrations were 
recorded outside of the sterile environment. One solution for this is obtaining a second batch of test strips that is used for the sterile experiments only. Therefore, one set of test strips may be used for preliminary glucose measurements, while the other may be used for glucose concentrations of solutions interacting with endothelial cells.

\subsubsection{Future Work in Support of this Thesis}

As pointed out by the literature researched for this thesis, there is a vast amount of research being conducted on the physiological mechanisms occurring within diabetic vasculature. These studies are focusing on primarily the expression and aggravation of particular genes or molecules caused by endothelial cell exposure to a hyperglycemic environment. As stated in a mentioned study, TUNEL staining can be conducted in order to evaluate apoptosis of endothelial cells exposed to a hyperglycemic environment for an extended period of time [145]. In addition to that, a study showed that exposure of HUVECs to high levels of glucose (which, according to this study, mimicked hyperglycemia), induced the expression of microRNA 221 (miR-221) - a short RNA structure that controls the expression of other genes [156]. This in turn, triggers the inhibition of the proto-oncogene c-Kit (or tyrosineprotein kinase Kit) - a protein receptor that is expressed on the surface of hematopoietic stem cells, which plays a crucial role in endothelial cell proliferation, differentiation, and migration [157]. This study, along with others, support the development of high glucose concentrated solutions in attempt to replicate a hyperglycemic environment, as well as experimentation with BAECs and HUVECs as reliable methods of evaluating endothelial cells dysfunction in a hyperglycemic environment. 
Additionally, researchers are evaluating the expression of intercellular adhesion molecule (ICAM) - 1, as well as the expression of vascular cellular adhesion molecule (VCAM) - 1 within endothelial cells exposed to a hyperglycemic environment. ICAM1 and VCAM 1 are both responsible for the adhesion of leukocytes to endothelial cells when they are activated [158, 159]. Studies have concluded that the hyperglycemic environment induced by diabetes promotes the expression of ICAM-1 and VCAM-1 along endothelial cells [160, 161]. This high expression of these adhesion molecules is most likely the cause of the high rate of in-stent restenosis in diabetics with CAD. Therefore, the next significant step of this thesis will be to evaluate whether the glycemic media solutions developed in this thesis affect cellular adhesion and proliferation within a BVM, and whether the solutions induce a similar expression of VCAM-1 and ICAM-1 on the endothelial cells within the BVM. The BVM can be analyzed by the following:

- Cellular morphology with a scanning electron microscope (SEM)

- Endothelial cell confluency and density with bisbenzimide (BBI) nucleus staining

- Blood vessel wall cellular thickness and cell confluency with hematoxylin and eosin (H\&E) staining

- Cellular apoptosis with TUNEL staining

- VCAM-1 \& ICAM-1 expression with VCAM-1 antibody and ICAM-1 antibody staining

While this entire thesis revolved around the affects hyperglycemia has on the cellular level, it is worth noting that hyperglycemia is not the only significant contributor to the detrimental vascular complications associated with diabetes. Recall, obesity is a pandemic 
caused by a combination of genetics and a modern environment where calorie-dense foods are widely available and inexpensive [187]. Coinciding with the obesity epidemic, type II diabetes mellitus is a related disorder with insulin resistance playing a crucial part in the pathogenesis of the disorder [188]. The cellular mechanisms contributing to the progression are poorly understood; however, previous studies by Randle et al. have hypothesized that the high availability of lipids as a source of fuel generate metabolic signals that impair the use of glucose through inhibition of key glycolytic enzymes [189]. Testing of this hypothesis has shown that lipid infusion raised plasma muscle lipids [190], and impaired oral glucose tolerance [191] and insulin-stimulated glucose disposal in healthy individuals [192, 193]. Thus, in healthy individuals, exposure to high concentrations of plasma fatty acids caused insulin resistance associated with an induced defect in either glucose transport or phosphorylation activity, not impairment of glycolysis [188]. Along with parallel studies, the results suggest that accumulation of lipids within muscle might be the cause of insulin resistance [188]. In addition, it has also been hypothesized that insulin resistance develops with the accumulation of fatty-acid metabolites (diacylglycerols) within insulin-responsive tissues [194]. The model of diacylglycerol-induced insulin resistance accounts for the insulin resistance seen in obesity and type II diabetes; moreover, reductions in in intracellular diacylglycerol content account for the improvements in insulin sensitivity after weight loss [195]. This drastic effect that lipid infiltration has on the development of insulin sensitivity encourages further research in its effect in type II diabetes; thus, it would be additionally beneficial to test the development of a hyperlipidemic cell media solutions and see the effect on endothelial cells in culture. 
If the endothelial cells within a hyperglycemic and hyperlipidemic environment exhibit characteristics similar to endothelial cells within a native diabetic blood vessel, then the goal of creating an in vitro diabetic blood vessel mimic for stent evaluation will be achieved.

This thesis is only a small part of the larger amount of research supporting both the development of blood vessel mimics for in vitro medical device evaluation at Cal Poly, as well as the understanding of mechanisms inducing many of the complications within diabetic patients at hospitals and labs worldwide. The lifestyle - and life expectancy - of diabetic patients has improved immensely since the significant discovery of insulin. New technologies and medications are continuously being studied and evaluated to further improve current methods of treatment. While this thesis was only able to study a minor part incorporating diseased blood vessel mimics with medical technologies treating diagnosed patients, this thesis was a new "gateway" to help improve not only the vascular devices used to treat CAD patients with diabetes, but hopefully to improve devices treating other diseases as well. 


\section{List of References}

1. Pyke DA. Preamble: the History of Diabetes. International Textbook of Diabetes Mellitus, 2nd ed. Oxford:1999;10-18.

2. Medvei C. The history of endocrinology, 2nd ed. New York: Patheonon 1993.

3. Bliss, M. The discovery of insulin. Edinburgh: Paul Harris Publishing, 1983.

4. Resenfeld L. Four Centuries of Clinical Chemistry led. Taylor and Francis Publishing New York: 1999, 157-158.

5. Cernea, S. Pozzilli, P. New potential treatments for protection of pancreatic B-cell function in Type 1 diabetes. DiabeticMedicine 2008;25(11):1259-1267.

6. Dotta, Francesco. Eisenbarth, George S. Immunopathogenesis of Type 1 Diabetes in Western Society. International Textbook of Diabetes Mellitus 2nd ed. Volume 1 1999.

7. Steck, Andrea K. Rewers, Marian J. Epidemiology and Geography of Type 1 Diabetes Mellitus. International Textbook of Diabetes Mellitus 3rd ed. John Wiley \& Sons Ltd, England. 2004;1:15-31.

8. Gale EA. The rise of childhood Type 1 diabetes in the 20th century. Diabetes 2002;51:3353-3361.

9. Gale EA. Spring harvest? Reflections on the rise of Type 1 diabetes. Diabetologia 2005;48:2445-2450.

10. Peng H, Hagopian W. Environmental factors in the development of Type 1 diabetes. Rev Endocr Metab Disord 2006;7:149-162.

11. Onakamo P, Vaananen S, Karvonen M, Tuomilehto J. Worldwide increase in incidence of type 1 diabetes-the analysis of the data published incidence trends. Diabetologia 1999;42(12):1395-1403.

12. Bottazzo GF, Gorsuch AN, Dean BM, Cudworth AG, Doniach D. Complement fixing islet cell antibodies in type 1 diabetes:possible monitors of active beta cell damage. Lancet 1980;42:1202-1209.

13. Baekkeskov S, Aanstoot $\mathrm{H}$, Chirstgau $\mathrm{S}$ et al. Identification of the $64 \mathrm{~K}$ autoantigen in insulin dependent diabetes as the GABA-synthesizing enzyme glutamic acid decarboxylase. Nature 1990; 347:151-156.

14. Pietropaolo M, Castano L, Babu S, Powers A, Eisenbarth GS. Molecular cloning and characterization of a novel neuroendocrine autoantigen (PM-1) related to type 1 diabetes. Diabetes 1992; 41 (supp. 1): 98A (abstract). 
15. Rabin DU, Pleasic SM, Shapiro JA et al. Islet cell antigen 512 is a diabetes-specific islet autoantigen related to protein tryosine phosphates. J Immunol 1994;152: 31833187.

16. Bingley PJ, Bonifacio E, Williams AJ, Genovese S, Bottazzo GF, Gale EA. Prediction of IDDM in the general population: strategies based on the combination of autoantibody markers. Diabetes 1997; 46 (11):1701-1710.

17. Li L, Yi Z, Tisch R, Wang B. Review: Immunotherapy of type 1 diabetes. Arch Immuno Ther Exp (Warsz) 2008.56:227-236.

18. Bleich D, Jackson RA, Soeldner JS, Eisenbarth GS. Analysis of metabolic progression to type 1 diabetes in islet cell antibody positive relatives of parents with type 1 diabets. Diabetes Care 1990;13:111-118.

19. LeRoith D. Beta-cell dysfunction and insulin resistance in type 2 diabetes: role of metabolic and genetic abnormalities. Am J Med 2002;8:1376-1382.

20. Hawkins M, Rossetti L. Insulin Resistance and Its Role in the Pathogenesis of Type 2 Diabetes. Joslin's Diabetes Mellitus 14th ed. Joslin Diabetes Center, Boston 2005;425-448.

21. Schmitz O, Orskov L, Lund S, et al. Glucose metabolism in chronic renal failure with reference to GH treatment or uremic chidren. J Pediatr Endocrinol 1993;6:53-59.

22. Isaksson B, Strommer L, Friess H, et al. Impaired insulin action on phosphatidylinositol 3-kinase activity and glucose transport in skeletal muscle of pancreatic cancer patients. Pancreas 2003;26:173-177.

23. Koopmans SJ, Ohman L, Haywood JR, et al. Seven days of euglycemic hyperinsulinemia induces insulin resistance for glucose metabolism but not hypertension, elevated catecholamine levels, or increased sodium retention in conscious normal rats. Diabetes 1997;46:1572-1578.

24. Ruvikumar B, Gerrard J, Dalla Man C, Firbank J, et al. Pioglitazone Decreases Fasting and Postprandial Endogenous Glucose Production in Proportion to Decrease in Hepatic Triglyceride Content. Diabetes 2008;57(9):2288-2295.

25. Bastard JP, Maachi M, Lagathy C, Kim MJ. Recent advances in the relationship between obesity, inflammation, and insulin resistance. European Cytokine Network 2006;17:4-12.

26. Yki-Jarvinen $\mathrm{H}$. The Insulin Resistance Syndrome. International Textbook of Diabetes Mellitus 3rd Ed. John Wiley \& Sons Ltd, England. 2004;1:359-373.

27. Havel PJ, Control of energy homeostasis and insulin action by adipocyte hormones: leptin, acylation stimulating protein, and adiponectin. Curr Opin Lipidol 2002;13:5159. 
28. Rajala MW, Scherer PE. Minireview: the adipocyte: at the crossroads of energy homeostasis, inflammation, and atherosclerosis. Endocrinology 2003;144:3765-3773.

29. Grimble RF. Inflammatory status and insulin resistance. Curr Opin Clin Nutr Metab Care 2002;5:551-559.

30. Festa A, D'Angostino R Jr, Howard G, et al. Chronic subclinical inflammation as part of the insulin resistance syndrome: the Insulin Resistance Atherosclerosis Study (IRAS). Circulation 2000;102:42-47.

31. Yki-Jarvinen H. Predication and prevention of non-insulin-dependent diabetes mellitus. In Williams G, Pickups J (eds) Textbook of Diabetes. Oxford: Blackwell, 2001; p 83.1

32. Kahn SE. Clinical review 135: the importance of beta-cell failure in the development and progression of type 2 diabetes. Journal of Clinical Endocrinology Metabolism 2001;86:4047-4058.

33. Leahy JL. B-cell dysfunction in type 2 diabetes mellitus. Joslin's Diabetes Mellitus 14th ed. Joslin Diabetes Center. Boston 2005;449-461.

34. Utzschneider KM, Kahn SE. B-cell dysfuction in type 2 diabetes. International Textbook of Diabetes Mellitus 3rd Ed. Volume 1. John Wiley \& Sons Ltd, England. 2004;1:375-388.

35. Sowers JR, Epstein M, Frohlich ED. Diabetes, hypertension, and cardiovascular disease: an update. Hypertension 2001;37:1053-1059.

36. McFarlane SI, Castro J, Kirpichnikov D, Sowers JR. Hypertension in diabetes mellitus. Joslin's Diabetes Mellitus 14th ed. Joslin Diabetes Center Boston:2005;970974.

37. Arauz-Panceco C, Parrott MA, Raskin P. Review: Treatment of hypertension in adults with diabetes. Diabetes Care 2003;26[Suppl 1]:S80-S82.

38. Umpierrez GE, Kitabchi AE. Diabetic ketoacidosis: risk factors and management strategies. Treat Endocrinology 2003;2:95-108.

39. Eledrisi MS, Alshanti MS, Shah MF, Brolosy B, Jaha N. Review: Overview of the diagnosis and management of diabetic ketoacidosis. American Journal of Medical Science 2006;331:243-251.

40. Wyckoff J, Abrahmson MJ. Diabetic ketoacidosis and hyperosmolar hyperglycemic state. Joslin's Diabetes Mellitus 14th ed. Joslin Diabetes Center Boston:2005;888899.

41. Woredekal Y. Early detection and treatment of diabetic nephropathy. Pediatric Endocrinology Review 2008;5(4):999-1004. 
42. Gruden G, Viberti G. Pathogenesis of Diabetic Nephropathy. Joslin's Diabetes Mellitus 14th ed. Joslin Diabetes Center. Boston, 2005;854-866.

43. Caramori ML, Fioretto P, Mauer M. Review: The need for early predictors of diabetic nephropathy risl: is albumin excretion rate sufficient? Diabetes 2000;49:1399-1408.

44. Steinke JM, Mauer M. Lessons learned from studies of the natural history of diabetic nephropathy in young type 1 diabetic patients. Pediatric Endocrinology Review 2008;5(4):958-963.

45. Krolewki AS, Warram JH. Epidemiology of late complications of diabetes: a basis for the development and eveluation of preventive programs. Joslin's Diabetes Mellitus 14th ed. Joslin Diabetes Center. Boston 2005;795-808.

46. Ritz E. Diabetic nephropathy. Saudi J Kidney Dis Transpl 2006;17:481-490.

47. Bash LD, Selvin E, Steffes M, Coresh J, Astor BC. Review: Poor glycemic control in diabetes and the risk of incident chronic kidney disease even in the absence of albuminuria and retinopathy: atherosclerosis risk in communities (ARIC) study. Arch Intern Med 2008;168:2440-2447.

48. Otto-Buczkowska E, Kazibutowska Z, Soltyk J, Machnica L. Review: Neuropathy and type 1 diabetes mellitus. Endokrynol Diabetol Chor Przemiany Materli Wieku Rosw 2008;14:109-116.

49. Obrosova IG. Diabetes and the peripheral nerve. Biochim Biophys Acta 2008 [ahead of print].

50. Di Carli MF, Bianco-Batlles D, Landa ME, et al. Effects of automatic neuropathy on coronary blood flow in patients with diabetes mellitus. Circulation 1999;100:813819.

51. Harati Y. Review: Diabetic neuropathies: unanswered questions. Neurol Clin 2007;25:303-317.

52. Johnstone MT, Nesto R. Diabetes mellitus and heart disease. Joslin's Diabetes Melltitus 14th ed. Joslin Diabetes Center Boston: 2005;976-998.

53. Pieske B, Wachter R. Review: Impact of diabetes and hypertension on the heart. Curr Opin Cardiol 2008;23:340-349.

54. Ancion A, Lanchellotti P, Pierard LA. Review: Congestive heart failure and diabetes mellitus. Rev Med Liege 2005;60:536-540.

55. Silber S, Herdeg C. Review: Drug-eluting stents for diabetic patients. A critical appraisal of the currently available data from randomized trials. Herz. 2008;33:196205. 
56. Lee W, Cheung A, Deanna C, Zinman B. Impact of diabetes on coronary artery disease in women and men: a meta-analysis of prospective studies. Diabetes Care 2000;23:962-968.

57. May O, Arildsen H, Damsgaard EM, Mickley H: Cardiovascular autonomic neuropathy in insulin-dependent diabetes mellitus: prevalence and estimated risk of coronary heart disease in the general population. Journal of Internal Medicine 2000;248:483-491.

58. Torffvit O, Lövestam-Adrian M, Agardh E, Agardh C-D: Nephropathy, but not retinopathy, is associated with the development of heart disease in type 1 diabetes: as 12-year observation study of 462 patients. Diabetes Med 2005 22:723-729.

59. Winocour PH, Durrington PN, Bhatnagar D, et al. Influence of early diabetic nephropathy on very low density lipoprotein (VLDL), intermediate density lipoprotein (IDL), and low density lipoprotein (LDL) composition. Atherosclerosis 1991;89:49-57.

60. Deckert T, Kofoed-Enevoldsen A, Norgaard K, et al. Microalbuminuria. Implications for micro- and macrovascular disease. Diabetes Care 1992;15:1181-1191.

61. Rewers M, Zaccaro D, D'Agostino R, Savage P, et al. Insulin sensitivity, insulinemia, and coronary artery disease. Diabetes Care 2004;27:781-787.

62. Daemen J, Kuck KH, Macaya C, LeGrand V, et al. Multivessel coronary revascularization in patients with and without diabetes mellitus: 3-year follow-up on the AFTS-II (Arterial revascularization therapies study-part II) trial. Journal of the American College of Cardiology 2008;52:1957-1967.

63. Carrozza J, Kuntz RE, Fishman RF, et al. Restenosis after arterial injury caused by coronary stenting in patients with diabetes mellitus. Ann Intern Med 1993;118:344349.

64. Kornowski R, Mintz GS, Kent KM, et al. Increased restenosis in diabetes mellitus after coronary interventions is due to exaggerated intimal hyperplasia. A serial intravascular ultrasound study. Circulation 1997;95:1366-1369.

65. Serrano-Rios M, Perez A, Saban-Ruiz J. Cardiac Complications in Diabetes: World Book of Diabetes in Practice. Princeton, NJ: Elsevier 1986;2:169-178.

66. Hirotani T, Kameda T, Kumamoto T, et al. Effects of coronary artery bypass grafting using internal mammary arteries for diabetic patients. Journal of the American College of Cardiology 1999;34:534-538.

67. Davies M, Kim JH, Klychkin ML, et al. Diabetes mellitus and experimental vein graft structure and function. Journal of Vascular Surgery 1994;19:1031-1043.

68. Singh SK, Desai ND, Petroff SD, Fremes SE, et al. The impact of diabetic status on coronary artery bypass graft patency. Circulation 2008;118:S222-S225. 
69. Kastrari A, Dibra A, Mehilli J, Mayer S, et al. Predictive factors of restenosis after coronary implantation of sirolimus or paclitaxel-eluting stents. Circulation 2006;113:2293-2300.

70. Cutlip DE, Chauhan MS, Baim DS, Ho KK, Popma JJ, Carrozza JP, Cohen DJ, Kuntz RE. Clinical restenosis after coronary stenting: perspectives from multicenter clinical trials. Journal of the American College of Cardiology 2002; 40: 2082-2089.

71. Costa MA, Simon DI. Molecular basis of restenosis and drug-eluting stents. Circulation 2005;111:2257-2273.

72. Forrester JS, Fishbein M, Helfant R, Fagin J. A paradigm for restenosis based on cell biology: clues for the development of new preventive therapies. Journal of the American College of Cardiology 1991;17:758-769.

73. Libby P, Shwartz D, Brogi E, Tanaka H, Clinton SK. A cascade model for restenosis: a special case of atherosclerosis progression. Circulation1992;86:47-52.

74. Ross R. The pathogenesis of atherosclerosis: a perspective for 1990s. Nature 1993;326:801-809.

75. Ceriello A, Falleti E, Bortolotti N, Motz E, Cavarape A, Russo A, Gonano F, Bartoli E. Increased circulating intracellular adhesion molecule-1 levels in type II diabetic patients: the possible role of metabolic control and oxidative stress. Metabolism 1996;45:498-501.

76. Otsuki M, Hashimoto K, Morimoto Y, Kishimoto T, Kasayama S. Ciculating vascular cell adhesion molecule-1 (VCAM-1) in atherosclerotic NIDM patients. Diabetes 1997;46:2096-2101.

77. Ceriello A, Falleti E, Motz E, Taboga C, Tonutti L, Ezsol Z, Gonano F, Bartoli E. Hyperglycemia-induced circulating ICAM-1 increase in diabetes mellitus: the possible role of oxidative stress. Horm Metab Res 1998;30:146-149.

78. Marfella R, Esposito K, Glunta R, Coppola G, De Angelis L, Farzati B, Paolisso G, Glugliano D. Circulating adhesion molecules in humans: role of hyperglycemia and hyperinsulinemia. Circulation 2000;101:2247-2251.

79. Quagliaro L, Piconi L, Assaloni R, Da Ros R, Maier A, Zuodar G, Ceriello A. Intermittent high glucose enhances ICAM-1, VCAM-1 and E-selectin expression in human umbilical vein endothelial cells in culture: The distinct role of protein kinase $\mathrm{C}$ and mitochondrial superoxide production. Atherosclerosis 2005;183:259-267.

80. Nyssen A, Legrand V, Scheen AJ. Review: Coronary drug-eluting stents in diabetic patients. Rev Med Suisse 2008;4:1806-1810. 
81. Dibra A, Kastrati A, Mehilli J, Pache J, Schuhlen H, Bechkerath N, Ulm K, Wessely R, Dirschinger J, Schomig A. Paclitaxel-eluting or sirolimus-eluting stents to prevent restenosis in diabetic patients. The New England Journal of Medicine 2005;353:663670 .

82. Kumbhani DJ, Bavry AA, Kamdar AR, Helton TJ, Bhatt DL. Review: The effect of drug-eluting stents on intermediate angiographic and clinical outcomes in diabetic patients: insghts from randomized clinical trials. American Heart Journal 2008; 155:640-647.

83. Stenestrand U, James SK, Lindback J, Frobert O, Carlsson J, Schersten F, Nilsson T, Lagerqvist B. Safety and efficacy of drug-eluting vs. bare metal stents in patients with diabetes mellitus: long-term of follow-up in the Swedish Coronary Angiography Registry (SCAAR). European Heart Journal 2009; [Epub ahead of print].

84. Carson J, Scholz P, Chen A, Peterson E, Gold J, Schneider S. Diabetes mellitus increases short-term mortality and morbidity in patients undergoing coronary artery bypass graft surgery. Journal of the American College of Cardiology 2002;40:418423.

85. Stettler C, Allemann S, Wandel S, Kastrati A, Morice C, Juni P et al. Drug eluting and bare metal stents in people with and without diabetes: collaborative network meta-analysis. British Medical Journal 2008;337:a1331.

86. Dibra A, Kastrati A, Mehilli J, Schomig A, et al. Paclitaxel-eluting or sirolimuseluting stents to prevent restenosis in diabetic patients. The New England Journal of Medicine 2005; 353:663-670.

87. Cardinal $\mathrm{K}$, et al. Tissue-engineered vascular grafts as in vitro blood vessel mimics for the evaluation of endothelialization of intravascular devices. Tissue Engineering 2006; 12:3431-3438.

88. American Diabetes Association www.diabetes.org

89. Eledrisi MS, Alshanti MS, Shah MF, Brolosy B, Jaha N. Review: Overview of the diagnosis and management of diabetic ketoacidosis. American Journal of Medical Science 2006;331:243-251.

90. Algenstaedt P, Schaefer C, Biermann T, Hamann A, Schwarzloh B, Greten H, Ruther W, Hansen-Algenstaedt N. Microvascular Alterations in Diabetic Mice Correlate With Level of Hyperglycemia. Diabetes 2003;52:542-549.

91. Haffner SM, Lehto S, Ronnemaa T, Pyorala K, Laakso M. Mortality from coronary heart disease in subjects with type 2 diabetes and in nondiabetic subjects with and without prior myocardial infarction. New England Journal of Medicine 1998;339:229-234.

92. Sheetz MJ, King GL. Molecular understanding of hyperglycemia's adverse effects for diabetic complications. JAMA 2002;288:2579-2588. 
93. Tepper OM, Galiano RD, Capla JM, Kalka C, Gagne PJ, Jacobowitz GR, Levine JP, Gurtner GC. Human endothelial progenitor cells from type II diabetics exhibit impaired proliferation, adhesion, and incorporation into vascular structures. Circulation 2002;106:2781-2786.

94. Antonetti DA, Lieth E, Barber AJ, Gardner TW. Molecular mechanisms of vascular permeability in diabetic retinopathy. Semin Ophthalmology 1999;14:240-248.

95. Panes J, Kurose I, Rodriguez-Vaca D, Anderson DC, Miyasaka M, Tso P, Granger DN. Diabetes exacerbates inflammatory responses to ischemia-reperfusion. Circulation 1996; 93:161-167.

96. Lutty GA, Cao J, McLeod DS. Relationship of polymorphonuclear leukocytes to capillary dropout in the human diabetic choroid. American Journal of Pathology 1997; 151:707-714.

97. Miyamoto K, Hiroshiba N, Tsujikawa A, Ogura Y. In vivo demonstration of increased leukocyte entrapment in retinal microcirculation of diabetic rats. Invest Ophthalmol Visual Science 1998;39:2190-2194.

98. Chiarelli F, Santilli F, Mohn A. Role of growth factors in the development of diabetic complications. Horm Res 2000;53:53-67.

99. Koya D, King GL: Protein kinase C activation and the development of diabetic complications. Diabetes 1998;47:859 -866.

100. Loomans CJM, de Koning EJP, Stall FJT, Rookmaaker MB, van Zonneveld A, et al. Endothelial progenitor cell dysfunction: a novel concept in the pathogenesis of vascular complications of type 1 diabetes. Diabetes 2004;53:195-199.

101. McMurry J. Organic Chemistry. Brooks/Cole, 1988. pp. 866.

102. Wade Jr. LG. Carbohydrates and Nucleic Acids. Organic Chemistry $6^{\text {th }}$ Ed. Pearson Prentice Hall, 2006. pp 1097-1152.

103. Wade Jr. LG. Ketones and Aldehydes. Organic Chemistry $6^{\text {th }}$ Ed. Pearson Prentice Hall, 2006. pp 805-869.

104. Ferraris RP. Dietary and developmental regulation of intestinal sugar transport. Biochemical Journal 2001;360: 265-276.

105. Tirosh A, Shai I, Tekes-Manova D, Rudich A, et al. Normal Fasting Plasma Glucose Levels and Type 2 Diabetics in Young Men. New England Journal of Medicine 2005; 353:1454-1462.

106. Besser GM, Thorner MO. Comprehensive Clinical Endocrinology, $3^{\text {rd }}$ ed. Philidelphia: Mosby, Elsevier Science Limited, 2002. 
107. Guyton AC, Hall JE. Insulin, glucagons, and diabetes mellitus. Textbook of Medical Physiology, $11^{\text {th }}$ ed. Elsevier Saunders, 2006. pp 968-969.

108. Roden M, Bernroider E. Hepatic glucose metabolism in humans - its role in health and disease. Best Pract Res Clin Endorinol Metab 2003:317-365.

109. Jiang G, Zhang BB. Glucagon and regulation of glucose metabolism. American Journal of Physiology of Endocrinology Metabolism 2003:284-E671.

110. Pilkis SJ, Granner DK. Molecular physiology of the regulation of hepatic glucogenesis and glycolysis. Annual Review of Physiology 1992:854-885.

111. Barrett EJ. Insulin's effect on glucose production: direct or indirect? Journal of Clinical Investigation 2003:111-434.

112. Kowluru A. Regulatory roles for small $\mathrm{G}$ proteins in pancreatic beta-cell: lessons from models of impaired insulin secretion. American Journal of Physiology of Endocrinology Metabolism 2003;285-E669.

113. Bryant NJ, Govers R, James DE. Regulated transport of the glucose transporter GLUT4. National Review of Molecular Cellular Biology 2002;3:267.

114. Efrat S. Regulation of insulin secretion: insights from engineered beta-cell lines. Ann NY Acad Sci 2004;1014-1088.

115. Pessin JE, Saltiel AR. Signaling pathways in insulin action: molecular targets of insulin resistance. Journal of Clinical Investigation 2000;106:165.

116. Cheathem B, Kahm CR. Insulin action and the insulin signaling network. Endocrinology Review 1995;16:117-142.

117. Kim YB, Nikoulina SE, Ciaraldi TP, Hentry RR, Kahn BB. Normal insulindependent activation of Akt/protein kniase B, witch diminished activation of phosphoinositide 3-kinase, in muscle in type 2 diabetes. Journal of Clinical Investigation 1999;104:733-741.

118. Shulman GI. Cellular mechanisms of insulin resistance. Journal of Clinical Investigation 2000;106:171-176.

119. Stenbit A, Tsao TS, Li J, Burcelin R, Greenen DL, Factor SM, Houseknecht K, Katz EB, Charron MJ. GLUT4 heterozygous knockout mice develop muscle insulin resistance and diabetes. Nature Medicine 1997;3:1096-1101.

120. Kadish AH, Litle RL, Sternberg JC. A new and rapid method for determination of glucose by measurement of rate of oxygen consumption. Clinical Chemistry 1968;14:116-131. 
121. Trajanoski Z, Brunner GA, Gferer RJ, Wach P, Pieber TR. Accuracy of home glucose meters during hypoglycemia. Diabetes Care 1996;19:1412-1415.

122. Weitgasser R, Gappmayer B, Pichler M. New portable glucose meters - analystical improvement compared with previous generation devices? Clinical Chemistry 1999;45:1821-1825.

123. Gibbs L. Visual test strips for glucose monitoring. www.betacheck.com

124. Marshall SM, Alberti KG. Assessment of a new visusal test strip for glucose monitoring. Diabetes Care 1983;6:543-547.

125. Brunner GA, Ellmerer M, Sendlhofer G, Wutte A, Piber TR, et al. Validation of home blood glucose meters with respect to clinical and analytical approaches. Diabetes Care 1998;21:585-590.

126. Poirier JY, Prieur NL, Campion L, Guilhem I, Allannic H, Maugendre D. Clinical and statistical evaluation of self-monitoring blood glucose meteres. Daibetes Care 1998;21:1919-1924.

127. Clarke WL, Cox D, Gonder-Frederick LA, Carter W, Pohl SL. Evaluating clinical accuracy of systems for self-monitoring of blood glucose. Diabetes Care 1987; 10:622-628.

128. Solnica B, Naskalski JW, Sieradzki J. Analytical performance of glucometeres used for routine glucose self-monitoring of diabetic patients. Clinica Chimica Acta 2003;331:29-35.

129. Chico A, Vidal-Rios P, Subira M, Novials A. The continuous glucose monitoring system is useful for detecting unrecognized hypoglycemias in patients with type 1 and type 2 diabetes but is not better than frequent capillary glucose measurements for improving metabolic control. Diabetes Care 2003;26:1153-1157.

130. Ristic JME, Herrtage ME, Walti-Lauger SMM, Slater LA, Church DB, Davison LJ, Catchpole B. Evaluation of a continuous glucose monitoring system in cats with diabetes mellitus. Journal of Feline Medicine \& Surgery 2005;7:153-162.

131. Bode BW, Gross TM, Thornton KR, Mastrototaro JJ. Continuous glucose monitoring used to adjust diabetes therapy improves glycosylated hemoglobin: a pilot study. Diabetes Research and Clinical Practice 1999;46:183-190.

132. Kaufman FR, Gibson LC, Halvorson M, Carpenter S, Fisher LK, Pitukcheewanont P. A pilot study of the continuous glucose monitoring system: clinical decisions and glycemic control after its use in pediatric type 1 diabetic subjects. Diabetes Care 2001;24:2030-2034. 
133. Aziz S, Jsiang YH. Comparative study of home blood glucose monitoring devices: Visidex, Chemstrip bG, Glucometer, and Accu-Check bG. Diabetes Care 1983;6:529-532.

134. Sensors for Medicine and Science, Inc. ${ }^{\circledR}$ Copyright $\odot 2009$.

135. Bequette W. Closed-loop regulation of blood glucose based on subcutaneous measurements. Diabetes Technology \& Therapeutics 2002;4:543-549.

136. Samann A, Fischbacher CH, Jagemann KU, Danzer K, Schuler J, Papenkordt L, Muller UA. Non-invasive blood glucose monitoring by means of near infrared spectroscopy: investivation of long-term accuracy and stability. Exp Clin Endocrinol Diabetes 2000;108:406-413.

137. Arnold MA. Non-invasive glucose monitoring. Current Opinion in Biotechnology 1996;7:46-49.

138. Sensys Medical. All Rights Reserved. Copyright @ 2005.

139. BAOEC: Bovine Aortic Endothelial Cells, n.d., Cell Applications, Inc., $<$ http://www.cellapplications.com/product_desc.php?id=89>.

140. Yamauchi T, Ohnaka K, Takayanagi R, Umeda F, Nawata H. Enhanced secretion of endothelin-1 by elevated glucose levels from cultured bovine aortic endothelial cells. FEBS Letters, Volume 267, Issue 1, 2 July 1990, Pages 16-18.

141. Du XL, Edelstein D, Dimmeler S, Sui Q, Brownlee M. Hyperglycemica inhibits endothelial nitric oxide synthase activity by posttranslational modification at the Akt site. J Clin. Invest. 2001; 108(9):1341.

142. Nishikawa T, Edelstein D, Brownlee M. Indirect Effects of High Glucose - The missing link: A single unifying mechanism for diabetic complications. Kidney International. 2000; 58:S26-S30.

143. Das EN, King GL. The role of protein kinase $\mathrm{C}$ activation and the vascular complications of diabetes. Pharmacol Res. 2007; 55: 498-510.

144. Human Umbilical Vein Endothelial Cells: HUVEC, n.d., Cell Applications, Inc. $<$ http://www.cellapplications.com/product_desc.php?id=82>

145. Yasuo I, Carling D, Ruderman N. Hyperglycemia-Induced Apoptosis in Human Umbilical Vein Endothelial Cells. Diabetes 2002; 51: 159-167.

146. Zhang Y, Shi H, Sun G, Li S, Xu X, Ye C, Li X, Wang S. High glucose induces dysfunction and apoptosis in endothelial cells: is the effect of high glucose persistence more important than concentration? Exp Clin Endocinol Diabetes. 2010 [Epub ahead of print]. 
147. Hardie DG, Carling D. The AMP-activated protein kinase - fuel gauge of the mammalian cell? Eur J Biochem. 1997; 246: 259-273.

148. Stefanelli C, Stanic I, Bonavita F, Flamigni F, Pignatti C, Guarnieri C, Caldarera CM. Inhibition of glucocorticoid-induced apoptosis with 5-aminoimidazole-4-carboxamide ribonucleoside, a cell-permeable activator of AMP-activated protein kinase. Biochem Biophys Res Commun. 1998; 243: 821-826.

149. Durante P, Gueuning MA, Darville MI, Hue L, Rousseau GG. Apoptosis induced by growth factor withdrawl in fibroblasts overproducing fructose 2,6-bisphosphate. FEBS Lett. 1999; 448: 239-243.

150. Ceriello A, Russo Pd, Amstad P, Cerutti P. High glucose induces antioxidant enzymes in human endothelial cells in culture. Evidence linking hyperglycemia and oxidative stress. Diabetes. 1996; 45: 471-477.

151. McCord JM, Fridovich I. Superoxide dismutase: the twenty years (1968-1988). Free Radic Biol Med. 1988; 5: 363-369.

152. Du Y, Kowluru A, Kern TS. PP2A contributes to endothelial death in high glucose: inhibition by benfotiamine. American Journal of Physiology 2010; 299: 610-617.

153. Daemen J, Kuck KH, Macaya C, LeGrand V, et al. Multivessel coronary revascularization in patients with and without diabetes mellitus: 3-year follow-up on the AFTS-II (Arterial revascularization therapies study-part II) trial. Journal of the American College of Cardiology 2008;52:1957-1967.

154. Marfella R, Esposito K, Glunta R, Coppola G, De Angelis L, Farzati B, Paolisso G, Glugliano D. Circulating adhesion molecules in himans: role of hyperglycemia and hyperinsulinemia. Circulation 2000;101:2247-2251.

155. Cardinal K, et al. Tissue-engineered vascular grafts as in vitro blood vessel mimics for the evaluation of endothelialization of intravascular devices. Tissue Engineering 2006; 12:3431-3438.

156. Li Y, Song YH, Li F, Yang T, Lu YW, Geng YJ. MicroRNA-221 regulates high glucose-induced endothelial dysfunction. Biochem Biophys Res Commun. 2009; 381: 81-83.

157. Andre C, Hampe A, Lachaume P, Martin E, Wang XP, Manus V, Hu WX, Galibert F. Sequence analysis of two genomic regions containing the KIT and the FMS receptor tyrosine kinase genes. Genomics 1997; 39: 216-226.

158. Yang L, Froio RM, Sciuto TE, Dvorak AM, Alon R, Luscinskas FW. ICAM-1 regulates neutrophil adhesion and transcellular migration of TNF-alpha-activated vascular endothelium under flow. Blood 2005; 106: 584-592.

159. Barreiro O, Yanez-Mo M, Serrador JM, Montoya MC, Vicente-Manzanares M, Tejedor R, Futhmayr H, Sanchez-Madrid F. Dynamic interaction of VCAM-1 and 
ICAM-1 with moesin and ezrin in a novel endothelial docking structure for adherent leukocytes. Journal of Cellular Biology 2002; 157: 1233-1245.

160. Bacun T, Glavas-Obrovac L, Belovari T, Mihaljević I, Hanich T, Belaj VG, Vcev A. Insulin administration in the mild hyperglycemia changes expression of proinflammatory adhesion molecules on human aortic endothelial cells. Coll Antropol. 2010; 34: 911-015.

161. Gustavsson C, Agardh CD, Zetterqvist AV, Nilsson J, Agardh E, Gomez MF. Vascular cellular adhesion molecule-1 (VCAM-1) expression in mice retinal vessels is affected by both hyperglycemia and hyperlipidemia.

162. Kwon JI, Kim YS, Cho AS, Kim JS, Jeong YS, et al. Origin of restenosis after drugeluting stent implantation in hyperglycemia is inflammatory cells and thrombus. Journal of Atherscelrosis and Thrombosis. Advanced publication accepted for publication January 28, 2011. Published online June 1, 2011.

163. Abdel-Wahab M, Khattab AA, Toelg R, Geist V, Liska B, Richardt G. Plaque characteristics of nonobstructive coronary lesions in diabetic patients: an intravascular ultrasound virtual histology analysis. Journal of Cardiovascular Medicine (Hagerstown) 2010; 11: 345-351.

164. Ogita M, Funayama H, Nakamura T, Sakakura K, Sugawara Y, Kubo N, Ako J, Ishikawa SE, Momomura S. Plaque characterization of non-culprit lesions by virtual histology intravascular ultrasound in diabetic patients: impact of renal function. Journal of Cardiology 2009; 54: 59-65.

165. Iakovou I, Schmidt T, Bonizzoni E, Ge L, Sangiorgi GM, Stankovic G, Airoldi F, Chieffo A, Monotorfano M, Carlino M, Michev I, Corvaja N, Briguori C, Gerckens $\mathrm{U}$, Grube E. Incidence, predictors, and outcome of thrombosis after successful implantation of drug-eluting stents. JAMA 2005; 293: 2126-2130.

166. Onuta G, Groenewegen HC, Klatter FA, Walther BM, Goris M, van Goor H, Roks AJ, Rozing J, de Smet BJ, Hillebrands JL. Long-term type 1 diabetes enhances instent restenosis after aortic stenting in diabetic-prone BB rats. Journal of Biomedical Biotechnology 2011; 396734.

167. Brownlee $\mathrm{M}$ et al. Biochemistry and molecular cell biology of diabetic complications. Nature 2001; 414: 813-820.

168. Lee AY, Chung SS. Contributions of polyol pathway to oxidative stress in diabetic cataract. FASEB J, 1999; 13: 23-30.

169. Engerman RL, Kern TS, Larson ME. Nerve conduction and aldose reductase inhibition during 5 years of diabetes or galactosaemia in dogs. Diabetologia 1994; 37: 141-144. 
170. Stitt AW et al. Advanced glycation end products (AGEs) co-localize with AGE receptors in the retinal vasculature of diabetic and of AGE-infused rats. American Journal of Pathology 1997; 150: 523-528.

171. Horie $\mathrm{K}$ et al. Immunohistochemical colocalization of glycoxidation products and lipid peroxidation products in diabetic renal glomerular lesions. Implications of glycooxidative stress in the pathogenesis of diabetic nephropathy. Journal of Clinical Investigation 1997; 100: 2995-2999.

172. Degenhardt TP, Thorpe SR, Baynes JW. Chemical modification of proteins by methylglyoxal. Cell Mol. Biol. 1998; 44: 1139-1145.

173. Wells-Knecht KJ et al. Mechanisms of autoxidative glycosylation: identification of glyoxal and arabinose as intermediates in the autoxidative modification of proteins by glucose. Biochemistry 1995; 34: 3702-3709.

174. Thornalley PJ. The glyoxalase system: new developments towards functional characterization of a metabolic pathway fundamental to biological life. Biochem J. 1990; 269: 1-11.

175. Giardino I, Edelstein D, Brownlee M. Nonenzymatic glycosylation in vitro and in bovine endothelial cells alters basic fibroblast growth factor activity. A model for intracellular glycosylation in diabetes. Journal of Clinical Investigation 1994; 94 : $110-117$.

176. Tanaka S, Avlgad G, Brodsky B, Elkenberry EF. Glycation induces expansion of the molecular packing of collagen. Journal of Molecular Biology 1988; 203: 495-505.

177. Huijberts MSP et al. Aminoguandidine treatment increses elasticity and decreases fluid filtration of large arteries from diabetic rats. Journal of Clinical Investigation 1993; 92: 1407-1411.

178. Haitoglou CS, Tsilibary EC, Brownlee M, Charonis AS. Altered cellular interactions between endothelial cells and nonenzymatically glucosylated laminin/type IV collagen. J. Biol. Chem. 1992; 267: 12404-12407.

179. Koya $\mathrm{D}$ et al. Characterization of protein kinase $\mathrm{C}$ beta isoform activation on the gene expression of transforming growth factor-beta, extracellular matrix components, and prostanoids in the glomeruli of diabetic rats. Journal of Clinical Investigation 1997; 100: 115-126.

180. Feener EP et al. Role of protein kinase $\mathrm{C}$ in glucose and angiotensin II-induced plasminogen activator inhibitor expression. Contrib. Nephrol. 1996; 118: 180-187.

181. Kolm-Litty V, Sauer U, Nerlich A, Lehmann R, Schleircher ED. High glucoseinduced transforming growth factor beta1 production is mediated by the hexosamine pathway in porcine glomerular mesangial cells. Journal of Clinical Investigation 1998; 101: 160-169. 
182. Marshall S, Bacote V, Traxinger RR. Discovery of a metabolic pathway mediating glucose-induced desensitization of the glucose transport system. Role of hexosamine biosynthesis in the induction of insulin resistance. J. Biol. Chem. 1991; 266: 47064712.

183. Hawkins $\mathrm{M}$ et al. Role of the glucosamine pathway in fat-induced insulin resistance. Journal of Clinical Investigation 1997; 99: 2173-2182.

184. Chen YQ et al. Sp1 sites mediate activation of the plasminogen activator inhibitor-1 promoter by glucose in vascular smooth muscle cells. J. Biol. Chem. 1998; 273: 8225-8231.

185. Du X et al. Hyperglycemia-induced mitochondrial superoxide overproduction activates the hexosamine pathway and induces plasminogen activator inhibitor-1 expression by increasing Sp1 glycosulation. Proc. Natl Acad. Sci. USA 2000; 97: 12222-12226.

186. Du X et al. Hyperglycemia inhibits endothelial nitric oxide synthase activity by posttranslational modification at the AKT site. Journal of Clinical Investigation (in the press).

187. Harris JL, Pomeranz JL, Lobstein T, Brownell KD. A crisis in the marketplace: how food marketing contributes to childhood obesity and what can be done. Annual Review of Public Health 2009; 30:211-225.

188. Samiel VT, Kitt FP, Shulman GI. Lipid-induced insulin resistance: unraveling the mechanism. Lancet 2010; 375: 2267-2277.

189. Randle PJ, Garland PB, Hales CN, Newsholme EA. The glucose fatty-acid cycle. Its role in insulin sensitivity and metabolic disturbances of diabetes mellitus. Lancet 1963; 1: 785-789.

190. Brechtel K, Dahl DB, Machann J et al. Fast elevation of the intramyocellular lipid content in the presence of circulating free fatty acids and hyperinsulinemia: a dynamic H-MRS study. Magn Reson Med 2001; 45: 179-183.

191. Felber JP, Golay A. Pathways from obesity to diabetes. Int J Obes Relat Metab Disord 2002; 26 (suppl 2): S39-S45.

192. Roden M, Price TB, Perseghin G et al. Mechanism of free fatty acid-induced insulin resistance in humans. Journal of Clinical Investigation 1996; 97: 2859-2865.

193. Boden G. Role of fatty acids in the pathogenesis of insulin resistance in NIDDM. Diabetes 1997; 46: 3-10.

194. Schulman G. Cellular mechanisms of insulin resistance. Journal of Clinical Investigation 2000; 106: 171-176. 
195. Petersen KF, Dufour S, Befroy D, Lehrke M, Hendler RE, Shulman GI. Reversal of nonalcoholic hepatic steatosis, hepatic insulin resistance, and hyperglycemia of

moderate weight reduction in patients with type 2 diabetes. Diabetes 2005; 54: 603608. 


\section{Appendix A: Results for Preliminary Testing to}

\section{Establish Method of Developing Glycemic Cell Media}

\section{Solutions}

Preliminary testing was conducted in order to analyze the simple procedure of adding specified amounts of Sigma G6152 D-(+)-Glucose to distilled water, and eventually base cell media. These experiments also helped determine the feasibility of certain methods of monitoring glucose concentrations, such as the blood glucose meter and visual blood glucose strips. The following tables show the results of these experiments.

Table 25 - Development of $100 \mathrm{mg} / \mathrm{dL}$ Solution Trial \#1

\begin{tabular}{|c|c|c|c|c|}
\hline \multicolumn{5}{|c|}{ Development of Hyperglycemic Solution Trial \#1 } \\
\hline Tube \# & $\begin{array}{c}\text { Expected } \\
\text { Glucose } \\
\text { Concentration } \\
(\mathbf{m g} / \mathbf{d L})\end{array}$ & $\begin{array}{c}\text { Volume of } \\
\text { Distilled } \mathrm{H}_{2} \mathrm{O} \\
\quad(\mathrm{mL})\end{array}$ & $\begin{array}{c}\text { Amount of } \\
\text { Sigma G6152 } \\
\text { D-(+)- Glucose } \\
\text { (g) }\end{array}$ & $\begin{array}{c}\text { Aviva Accu- } \\
\text { Chek Blood } \\
\text { Glucose Meter } \\
\text { Measurement } \\
\text { (mg/dL) }\end{array}$ \\
\hline 1 & \multirow{4}{*}{$100 \mathrm{mg} / \mathrm{dL}$} & 20 & 0.2004 & 76 \\
\hline 2 & & 20 & 0.2002 & 129 \\
\hline 3 & & 20 & 0.2001 & 162 \\
\hline 4 & & 20 & 0.206 & 35 \\
\hline
\end{tabular}


Table 26 - Development of $100 \mathrm{mg} / \mathrm{dL} \& 180 \mathrm{mg} / \mathrm{dL}$ Solutions Trial \#2

\begin{tabular}{|c|c|c|c|c|}
\hline \multicolumn{5}{|c|}{ Development of $100 \mathrm{mg} / \mathrm{dL} \& 180 \mathrm{mg} / \mathrm{dL}$ Solutions Trial \#2 } \\
\hline $\begin{array}{c}\text { Expected } \\
\text { Glucose } \\
\text { Concentration } \\
(\mathbf{m g} / \mathbf{d L})\end{array}$ & $\begin{array}{c}\text { Volume of } \\
\text { Distilled } \\
\mathrm{H}_{2} \mathrm{O}(\mathrm{mL})\end{array}$ & $\begin{array}{c}\text { Amount of } \\
\text { Sigma } \\
\text { G6152 } \\
\text { D-(+)- } \\
\text { Glucose } \\
\text { (g) }\end{array}$ & Time (min) & $\begin{array}{c}\text { Aviva Accu- } \\
\text { Chek Blood } \\
\text { Glucose Meter } \\
\text { Measurement } \\
\text { (mg/dL) }\end{array}$ \\
\hline \multirow{6}{*}{100} & \multirow{6}{*}{10} & \multirow{6}{*}{0.011} & 5 & 17 \\
\hline & & & 10 & 71 \\
\hline & & & 15 & 83 \\
\hline & & & 20 & 106 \\
\hline & & & 25 & 117 \\
\hline & & & 30 & 129 \\
\hline \multirow{6}{*}{180} & \multirow{6}{*}{10} & \multirow{6}{*}{0.0187} & 5 & 136 \\
\hline & & & 10 & 201 \\
\hline & & & 15 & $\mathrm{x}$ \\
\hline & & & 20 & $\mathrm{x}$ \\
\hline & & & 25 & $\mathrm{x}$ \\
\hline & & & 30 & $\mathrm{x}$ \\
\hline
\end{tabular}

$x$ Ran out of glucose test strips 
Table 27 - Development of 100 mg/dL Normal Glycemic \& 400 mg/dL Hyperglycemic Solutions Trial \#3

\begin{tabular}{|c|c|c|c|}
\hline \multicolumn{4}{|c|}{ Development of $100 \mathrm{mg} / \mathrm{dL}$ Normal Glycemic \& $400 \mathrm{mg} / \mathrm{dL}$ Hyperglycemic Solutions Trial \#3 } \\
\hline $\begin{array}{c}\text { Expected Glucose } \\
\text { Concentration }(\mathbf{m g} / \mathbf{d L})\end{array}$ & $\begin{array}{c}\text { Amount of } \\
\text { Sigma G6152 } \\
\text { D-(+)- Glucose } \\
\text { (g) added to } 10 \\
\text { mL } \mathrm{H}_{2} \mathrm{O} \\
\end{array}$ & Time (min) & $\begin{array}{l}\text { Blood Glucose Meter } \\
\text { Measurement (mg/dL) }\end{array}$ \\
\hline \multirow{6}{*}{100} & \multirow{6}{*}{0.0134} & 5 & 74 \\
\hline & & 10 & 130 \\
\hline & & 20 & 152 \\
\hline & & 30 & 217 \\
\hline & & 40 & 251 \\
\hline & & Average & 164.8 \\
\hline \multirow{9}{*}{460} & \multirow{9}{*}{0.00165} & 5 & 445 \\
\hline & & 10 & 467 \\
\hline & & 20 & 480 \\
\hline & & 30 & 515 \\
\hline & & 40 & 485 \\
\hline & & 40 & 496 \\
\hline & & 40 & 501 \\
\hline & & 45 & 479 \\
\hline & & Average & 483.5 \\
\hline
\end{tabular}


Table 28 - Development of $100 \mathrm{mg} / \mathrm{dL}$ Normal Glycemic \& $350 \mathrm{mg} / \mathrm{dL}$ Hyperglycemic Solutions Trial \#4

\begin{tabular}{|c|c|c|c|c|}
\hline \multicolumn{5}{|c|}{ Development of $100 \mathrm{mg} / \mathrm{dL}$ Normal Glycemic \& $350 \mathrm{mg} / \mathrm{dL}$ Hyperglycemic Solutions Trial \#4 } \\
\hline $\begin{array}{c}\text { Expected Glucose } \\
\text { Concentration } \\
\text { (mg/dL) }\end{array}$ & $\begin{array}{l}\text { Amount Glucose } \\
\text { Added to Media } \\
\text { (g) }\end{array}$ & $\begin{array}{l}\text { Volume of Gibco } \\
\text { Medium } 199(\mathrm{~mL})\end{array}$ & Time (min) & $\begin{array}{l}\text { Blood Glucose Meter } \\
\text { Measurement } \\
\text { (mg/dL) }\end{array}$ \\
\hline \multirow{10}{*}{100} & \multirow{5}{*}{0.0506} & \multirow{5}{*}{50} & 30 & 156 \\
\hline & & & 60 & 189 \\
\hline & & & 120 & 198 \\
\hline & & & 240 & 201 \\
\hline & & & 720 & 215 \\
\hline & \multirow{5}{*}{0.1061} & \multirow{5}{*}{100} & 30 & 148 \\
\hline & & & 60 & 165 \\
\hline & & & 120 & 184 \\
\hline & & & 240 & 209 \\
\hline & & & 720 & 206 \\
\hline \multirow{10}{*}{350} & \multirow{5}{*}{0.1758} & \multirow{5}{*}{50} & 30 & 318 \\
\hline & & & 60 & 397 \\
\hline & & & 120 & 413 \\
\hline & & & 240 & 481 \\
\hline & & & 720 & 435 \\
\hline & \multirow{5}{*}{0.3507} & \multirow{5}{*}{100} & 30 & 388 \\
\hline & & & 60 & 417 \\
\hline & & & 120 & 451 \\
\hline & & & 240 & 466 \\
\hline & & & 720 & 458 \\
\hline
\end{tabular}




\section{Appendix B: Results for Experimentation to}

\section{Determine Most Feasible Method of Measuring}

\section{Glucose Concentration of Developed Glycemic Cell}

\section{Media Solutions}

A set of experiments was conducted to evaluate the precision and accuracy of the Aviva Accu-Chek blood glucose meter and the Glucoflex-R visual blood glucose strips among glycemic solutions of $200 \mathrm{mg} / \mathrm{dL}, 350 \mathrm{mg} / \mathrm{dL}$, and $500 \mathrm{mg} / \mathrm{dL}$ glucose concentrations. The

following tables show the results of these experiments. Notice the calculated percent error as well as the standard deviation between the Aviva Accu-Chek blood glucose meter and the Glucoflex-R visual blood glucose test strips. 
Table 29 - Method of Monitoring 200 mg/dL Normal Glycemic Environment Test Results

\begin{tabular}{|c|c|c|c|c|c|}
\hline \multicolumn{6}{|c|}{ Method of Monitoring 200 mg/dL Normal Glycemic Environment Test Results } \\
\hline $\begin{array}{c}\text { Method of } \\
\text { Measurement }\end{array}$ & Time (hours) & $\begin{array}{c}\text { Calibration } \\
\text { Value }(\mathrm{mg} / \mathrm{dL})\end{array}$ & $\begin{array}{c}\text { Measured } \\
\text { Glucose } \\
\text { Concentration } \\
\text { Solution } 1 \\
\text { (mg/dL) } \\
\end{array}$ & $\begin{array}{c}\text { Measured } \\
\text { Glucose } \\
\text { Concentration } \\
\text { Solution } 2 \\
\text { (mg/dL) } \\
\end{array}$ & $\begin{array}{c}\text { Measured } \\
\text { Glucose } \\
\text { Concentration } \\
\text { Solution } 3 \\
(\mathrm{mg} / \mathrm{dL}) \\
\end{array}$ \\
\hline \multirow{13}{*}{$\begin{array}{c}\text { Aviva Accu- } \\
\text { Chek Blood } \\
\text { Glucose Meter }\end{array}$} & 1 & 437 & 189 & 169 & 174 \\
\hline & 2 & 444 & 200 & 200 & 196 \\
\hline & 3 & 456 & 204 & 199 & 190 \\
\hline & 4 & 446 & 209 & 195 & 188 \\
\hline & 5 & 452 & 206 & 192 & 192 \\
\hline & 6 & 443 & 200 & 200 & 197 \\
\hline & 12 & 439 & 215 & 207 & 208 \\
\hline & 24 & 447 & 209 & 215 & 212 \\
\hline & 36 & 455 & 213 & 213 & 207 \\
\hline & 48 & 441 & 202 & 195 & 189 \\
\hline & Average & 446.56 & 204.7 & 198.5 & 195.3 \\
\hline & St. Dev. & 6.55 & 7.54 & 12.89 & 11.38 \\
\hline & \% Error & -0.77 & 2.35 & -0.75 & -2.35 \\
\hline $\begin{array}{c}\text { Method of } \\
\text { Measurement }\end{array}$ & Time (hours) & $\begin{array}{c}\text { Calibration } \\
\text { Value }(\mathrm{mg} / \mathrm{dL})\end{array}$ & $\begin{array}{c}\text { Measured } \\
\text { Glucose } \\
\text { Concentration } \\
\text { Solution } 1 \\
\text { (mg/dL) } \\
\end{array}$ & $\begin{array}{c}\text { Measured } \\
\text { Glucose } \\
\text { Concentration } \\
\text { Solution } 2 \\
\text { (mg/dL) } \\
\end{array}$ & $\begin{array}{c}\text { Measured } \\
\text { Glucose } \\
\text { Concentration } \\
\text { Solution 3 } \\
(\mathrm{mg} / \mathrm{dL}) \\
\end{array}$ \\
\hline \multirow{13}{*}{$\begin{array}{c}\text { Glucoflex-R } \\
\text { Visual Blood } \\
\text { Glucose Test } \\
\text { Strips }\end{array}$} & 1 & 400 & 150 & 160 & 500 \\
\hline & 2 & N/A & 200 & 160 & 300 \\
\hline & 3 & $\mathrm{~N} / \mathrm{A}$ & 200 & 120 & 200 \\
\hline & 4 & $\mathrm{~N} / \mathrm{A}$ & 300 & 160 & 200 \\
\hline & 5 & $\mathrm{~N} / \mathrm{A}$ & 200 & 160 & 200 \\
\hline & 6 & N/A & 180 & 200 & 160 \\
\hline & 12 & N/A & 200 & 160 & 300 \\
\hline & 24 & $\mathrm{~N} / \mathrm{A}$ & 300 & 160 & 800 \\
\hline & 36 & N/A & 160 & 500 & 300 \\
\hline & 48 & N/A & 160 & 160 & 160 \\
\hline & Average & N/A & 205 & 194 & 312 \\
\hline & St. Dev. & N/A & 53.59 & 109.16 & 199.38 \\
\hline & $\%$ Error & N/A & 2.50 & -3.00 & 56.00 \\
\hline
\end{tabular}


Table 30 - Method of Monitoring 350 mg/dL Hyperglycemic Environment Test Results

\begin{tabular}{|c|c|c|c|c|c|}
\hline \multicolumn{6}{|c|}{ Method of Monitoring $350 \mathrm{mg} / \mathrm{dL}$ Hyperglycemic Environment Test Results } \\
\hline $\begin{array}{c}\text { Method of } \\
\text { Measurement }\end{array}$ & Time (hours) & $\begin{array}{c}\text { Calibration } \\
\text { Value }(\mathrm{mg} / \mathrm{dL})\end{array}$ & $\begin{array}{c}\text { Measured } \\
\text { Glucose } \\
\text { Concentration } \\
\text { Solution 1 } \\
\text { (mg/dL) } \\
\end{array}$ & $\begin{array}{c}\text { Measured } \\
\text { Glucose } \\
\text { Concentration } \\
\text { Solution } 2 \\
\text { (mg/dL) } \\
\end{array}$ & $\begin{array}{c}\text { Measured } \\
\text { Glucose } \\
\text { Concentration } \\
\text { Solution } 3 \\
(\mathrm{mg} / \mathrm{dL}) \\
\end{array}$ \\
\hline \multirow{13}{*}{$\begin{array}{c}\text { Aviva Accu- } \\
\text { Chek Blood } \\
\text { Glucose Meter }\end{array}$} & 1 & 446 & 271 & 137 & 133 \\
\hline & 2 & 467 & 276 & 226 & 257 \\
\hline & 3 & 433 & 292 & 299 & 302 \\
\hline & 4 & 441 & 310 & 337 & 342 \\
\hline & 5 & 457 & 352 & 359 & 349 \\
\hline & 6 & 425 & 355 & 350 & 359 \\
\hline & 12 & 449 & 373 & 354 & 360 \\
\hline & 24 & 458 & 363 & 341 & 356 \\
\hline & 36 & 461 & 357 & 366 & 365 \\
\hline & 48 & 453 & 387 & 371 & 372 \\
\hline & Average $(t=4)$ & 449.00 & 356.71 & 354.00 & 357.57 \\
\hline & St. Dev. $(t=4)$ & 13.06 & 23.91 & 11.54 & 9.91 \\
\hline & $\%$ Error & -0.22 & 1.92 & 1.14 & 2.16 \\
\hline \multirow{13}{*}{$\begin{array}{c}\text { Glucoflex-R } \\
\text { Visual Blood } \\
\text { Glucose Test } \\
\text { Strips }\end{array}$} & 1 & 300 & 300 & 200 & 200 \\
\hline & 2 & N/A & 300 & 300 & 250 \\
\hline & 3 & N/A & 500 & 300 & 300 \\
\hline & 4 & N/A & 300 & 500 & 300 \\
\hline & 5 & N/A & 400 & 300 & 300 \\
\hline & 6 & N/A & 250 & 300 & 250 \\
\hline & 12 & $\mathrm{~N} / \mathrm{A}$ & 200 & 250 & 300 \\
\hline & 24 & N/A & 800 & 500 & 300 \\
\hline & 36 & N/A & 300 & 200 & 300 \\
\hline & 48 & N/A & 300 & 200 & 300 \\
\hline & Average $(t=4)$ & N/A & 364.29 & 321.43 & 292.86 \\
\hline & St. Dev. $(t=4)$ & N/A & 201.48 & 128.64 & 18.90 \\
\hline & $\%$ Error & N/A & 4.08 & -8.16 & -16.33 \\
\hline
\end{tabular}


Table 31 - Method of Monitoring 500 mg/dL Hyperglycemic Environment Test Results

\begin{tabular}{|c|c|c|c|c|c|}
\hline \multicolumn{6}{|c|}{ Method of Monitoring 500 mg/dL Hyperglycemic Environment Test Results } \\
\hline $\begin{array}{c}\text { Method of } \\
\text { Measurement }\end{array}$ & Time (hours) & $\begin{array}{c}\text { Calibration } \\
\text { Value }(\mathrm{mg} / \mathrm{dL})\end{array}$ & $\begin{array}{c}\text { Measured } \\
\text { Glucose } \\
\text { Concentration } \\
\text { Solution 1 } \\
\text { (mg/dL) } \\
\end{array}$ & $\begin{array}{c}\text { Measured } \\
\text { Glucose } \\
\text { Concentration } \\
\text { Solution } 2 \\
\text { (mg/dL) } \\
\end{array}$ & $\begin{array}{c}\text { Measured } \\
\text { Glucose } \\
\text { Concentration } \\
\text { Solution } 3 \\
(\mathrm{mg} / \mathrm{dL}) \\
\end{array}$ \\
\hline \multirow{13}{*}{$\begin{array}{c}\text { Aviva Accu- } \\
\text { Chek Blood } \\
\text { Glucose Meter }\end{array}$} & 1 & 447 & 177 & 164 & 165 \\
\hline & 2 & 451 & 247 & 253 & 251 \\
\hline & 3 & 450 & 303 & 313 & 299 \\
\hline & 4 & 439 & 289 & 395 & 392 \\
\hline & 5 & 444 & 459 & 457 & 452 \\
\hline & 6 & 453 & 469 & 466 & 460 \\
\hline & 12 & 448 & 479 & 472 & 461 \\
\hline & 24 & 437 & 511 & 483 & 468 \\
\hline & 36 & 448 & 528 & 473 & 475 \\
\hline & 48 & 454 & 514 & 485 & 483 \\
\hline & Average $(\mathrm{t}=5)$ & 447.33 & 493.33 & 472.67 & 466.50 \\
\hline & St. Dev. $(t=5)$ & 6.250 & 27.990 & 10.482 & 11.221 \\
\hline & $\%$ Error & $\mathrm{N} / \mathrm{A}$ & -1.333 & -5.467 & -6.700 \\
\hline \multirow{13}{*}{$\begin{array}{c}\text { Glucoflex-R } \\
\text { Visual Blood } \\
\text { Glucose Test } \\
\text { Strips }\end{array}$} & 1 & 400 & 400 & 400 & 400 \\
\hline & 2 & 500 & 500 & 300 & 500 \\
\hline & 3 & $\mathrm{~N} / \mathrm{A}$ & 500 & 500 & 500 \\
\hline & 4 & N/A & 800 & 500 & 300 \\
\hline & 5 & N/A & 500 & 500 & 500 \\
\hline & 6 & N/A & 800 & 300 & 300 \\
\hline & 12 & N/A & 200 & 400 & 300 \\
\hline & 24 & N/A & 500 & 400 & 400 \\
\hline & 36 & N/A & 500 & 300 & 500 \\
\hline & 48 & $\mathrm{~N} / \mathrm{A}$ & 500 & 400 & 400 \\
\hline & Average $(\mathrm{t}=5)$ & N/A & 500.00 & 383.33 & 400.00 \\
\hline & St. Dev. $(t=5)$ & N/A & 189.737 & 75.277 & 89.443 \\
\hline & $\%$ Error & N/A & 0.000 & -23.333 & -20.000 \\
\hline
\end{tabular}




\section{Appendix C: Results for Experimentation of BAECs \& HUVECs Exposed to $100 \mathrm{mg} / \mathrm{dL}$ Normal Glycemic}

\section{Cell Media Solutions \& 400 mg/dL Hyperglycemic}

\section{Cell Media Solutions}

The following tables show the results of the six experiments conducted with BAECs and HUVECs. The purpose of these tests was to evaluate the glucose consumption of the cells in the $100 \mathrm{mg} / \mathrm{dL}$ and $400 \mathrm{mg} / \mathrm{dL}$ glycemic cell media solutions. Additionally, the change in glucose concentrations was calculated in order to analyze the rate of glucose consumption between the different times of evaluation ( $0,24,48$, and 72 hours). 
Table 32 - BAEC Glucose Consumption Comparative Study Experiment \#1

\begin{tabular}{|c|c|c|c|c|}
\hline \multirow{5}{*}{ Time } & $\begin{array}{c}\text { Expected Glucose } \\
\text { Concentration } \\
(\mathbf{m g} / \mathbf{d L})\end{array}$ & Well \#1 & Well \#2 & Well \#3 \\
\cline { 3 - 5 } & & 99 & 98 & 99 \\
\hline \multirow{2}{*}{0} & 100 & 416 & 398 & 414 \\
\cline { 2 - 5 } & 400 & 100 & 103 & 98 \\
\hline \multirow{2}{*}{24} & 100 & 335 & 365 & 341 \\
\cline { 2 - 5 } & 400 & 94 & 91 & 94 \\
\hline \multirow{2}{*}{48} & 100 & 355 & 355 & 930 \\
\cline { 2 - 5 } & 400 & 96 & 95 & 323 \\
\hline \multirow{2}{*}{72} & 100 & 347 & 340 & 95 \\
\cline { 2 - 5 } & 400 & & & \\
\hline
\end{tabular}

Table 33 - BAEC Glucose Consumption Comparative Study Experiment \#1 - Well $\Delta \mathrm{mg} / \mathrm{dL}$ per Time Chart

\begin{tabular}{|c|c|c|c|c|c|c|c|c|}
\hline \multicolumn{9}{|c|}{$\begin{array}{l}\text { BAEC Glucose Consumption Comparative Study Experiment \#1 - } \\
\text { Well } \Delta \text { mg/dL per Time Chart }\end{array}$} \\
\hline \multicolumn{9}{|c|}{$100 \mathrm{mg} / \mathrm{dL}$} \\
\hline $\begin{array}{c}\text { Well } \\
\#\end{array}$ & $\underset{\left(\mathbf{t}_{0}-\mathbf{t}_{72}\right)}{\Delta \mathbf{m g} / \mathbf{d L}}$ & $\begin{array}{c}\operatorname{Avg} \Delta \\
\mathbf{m g} / \mathbf{d L} \\
\left(\mathbf{t}_{0}-\mathbf{t}_{\mathbf{7 2}}\right)\end{array}$ & $\underset{\left(t_{0}-t_{24}\right)}{\Delta \mathbf{m g}}$ & $\begin{array}{l}\operatorname{Avg} \Delta \\
\mathbf{m g} / \mathbf{d L} \\
\left(\mathbf{t}_{0}-\mathbf{t}_{24}\right)\end{array}$ & $\begin{array}{l}\Delta \mathbf{m g} / \mathbf{d L} \\
\left(\mathbf{t}_{24}-\mathbf{t}_{48}\right)\end{array}$ & $\begin{array}{c}\operatorname{Avg} \Delta \\
\text { mg/dL } \\
\left(\mathbf{t}_{24}-\mathbf{t}_{48}\right)\end{array}$ & $\begin{array}{c}\Delta \mathrm{mg} / \mathrm{dL} \\
\left(\mathrm{t}_{48}-\mathbf{t}_{72}\right)\end{array}$ & $\begin{array}{c}\operatorname{Avg} \Delta \\
\operatorname{mg} / d L \\
\left(t_{48}-t_{72}\right)\end{array}$ \\
\hline 1 & 3 & \multirow{3}{*}{3.333} & -1 & \multirow{3}{*}{-1.667} & 6 & \multirow{3}{*}{7.333} & -2 & \multirow{3}{*}{-2.333} \\
\hline 2 & 3 & & -5 & & 12 & & -4 & \\
\hline 3 & 4 & & 1 & & 4 & & -1 & \\
\hline \multicolumn{9}{|c|}{$400 \mathrm{mg} / \mathrm{dL}$} \\
\hline $\begin{array}{c}\text { Well } \\
\#\end{array}$ & $\begin{array}{c}\Delta \mathrm{mg} / \mathrm{dL} \\
\left(\mathrm{t}_{\mathbf{0}}-\mathrm{t}_{\mathbf{7 2}}\right)\end{array}$ & $\begin{array}{c}\operatorname{Avg} \Delta \\
\mathbf{m g} / \mathbf{d L} \\
\left(\mathrm{t}_{0}-\mathrm{t}_{72}\right)\end{array}$ & $\underset{\left(t_{0}-t_{24}\right)}{\Delta m g / d L}$ & $\begin{array}{l}\operatorname{Avg} \Delta \\
\mathbf{m g} / \mathbf{d L} \\
\left(\mathrm{t}_{\mathbf{0}}-\mathbf{t}_{24}\right)\end{array}$ & $\begin{array}{c}\Delta \mathrm{mg} / \mathrm{dL} \\
\left(\mathrm{t}_{24}-\mathbf{t}_{48}\right)\end{array}$ & $\begin{array}{c}\operatorname{Avg} \Delta \\
\text { mg/dL } \\
\left(\mathbf{t}_{24}-\mathbf{t}_{48}\right)\end{array}$ & $\begin{array}{c}\Delta \mathrm{mg} / \mathrm{dL} \\
\left(\mathrm{t}_{48}-\mathbf{t}_{72}\right)\end{array}$ & $\begin{array}{c}\operatorname{Avg} \Delta \\
\mathbf{m g} / \mathbf{d L} \\
\left(\mathbf{t}_{48}-\mathbf{t}_{\mathbf{7 2}}\right)\end{array}$ \\
\hline 1 & 69 & \multirow{3}{*}{72.667} & 81 & \multirow{3}{*}{62.333} & -20 & \multirow{3}{*}{0.333} & 8 & \multirow{3}{*}{10.000} \\
\hline 2 & 58 & & 33 & & 10 & & 15 & \\
\hline 3 & 91 & & 73 & & 11 & & 7 & \\
\hline
\end{tabular}


Table 34 - HUVEC Glucose Consumption Comparative Study Experiment \#1

\begin{tabular}{|c|c|c|c|c|}
\hline \multicolumn{5}{|c|}{ HUVEC Glucose Consumption Comparative Study Experiment \#1 } \\
\hline \multirow{3}{*}{ Time } & $\begin{array}{c}\text { Expected Glucose } \\
\text { Concentration } \\
\text { (mg/dL) }\end{array}$ & Glucose Measurements via Accu-Check Glucose Meter (mg/dL) \\
\cline { 3 - 5 } & 100 & 103 & Well \#2 & Well \#3 \\
\hline \multirow{2}{*}{0} & 400 & 258 & 106 & 100 \\
\cline { 2 - 5 } & 100 & 90 & 222 & 260 \\
\hline \multirow{2}{*}{24} & 400 & 229 & 96 & 96 \\
\cline { 2 - 5 } & 100 & 89 & 226 & 90 \\
\hline \multirow{2}{*}{48} & 400 & 223 & 88 & 224 \\
\hline \multirow{2}{*}{72} & 100 & 90 & 91 & 90 \\
\cline { 2 - 5 } & 400 & 234 & 224 & 223 \\
\hline
\end{tabular}

Table 35 - HUVEC Glucose Consumption Comparative Experiment Trial \#1 - Well $\Delta$ mg/dL per Time Chart

\begin{tabular}{|c|c|c|c|c|c|c|c|c|c|}
\hline \multicolumn{7}{|c|}{ HUVEC Glucose Consumption Comparative Experiment Trial \#1 - } \\
Well $\Delta$ mg/dL per Time Chart
\end{tabular}


Table 36 - BAEC Glucose Consumption Comparative Study Experiment \#2

\begin{tabular}{|c|c|c|c|c|}
\hline \multicolumn{5}{|c|}{ BAEC Glucose Consumption Comparative Study Experiment \#2 } \\
\hline \multirow{3}{*}{ Time } & \multirow{2}{*}{$\begin{array}{c}\text { Expected Glucose } \\
\text { Concentration } \\
(\mathbf{m g} / \mathbf{d L})\end{array}$} & Glucose Measurements via Accu-Check Glucose Meter (mg/dL) \\
\cline { 3 - 5 } & 100 & 90 & Well \#2 & Well \#3 \\
\hline \multirow{2}{*}{0} & 400 & 289 & 85 & 89 \\
\cline { 2 - 5 } & 100 & 89 & 277 & 297 \\
\hline \multirow{2}{*}{24} & 400 & 288 & 88 & 90 \\
\cline { 2 - 5 } & 100 & 83 & 263 & 281 \\
\hline \multirow{2}{*}{48} & 400 & 253 & 253 & 266 \\
\hline \multirow{2}{*}{72} & 100 & 75 & 70 & 70 \\
\cline { 2 - 5 } & 400 & 252 & 241 & 252 \\
\hline
\end{tabular}

Table 37 - BAEC Glucose Consumption Comparative Study Experiment \#2 - Well $\Delta$ mg/dL per Time Chart

\begin{tabular}{|c|c|c|c|c|c|c|c|c|}
\hline \multicolumn{9}{|c|}{$\begin{array}{c}\text { BAEC Glucose Consumption Comparative Study Experiment \#2 - } \\
\text { Well } \Delta \text { mg/dL per Time Chart }\end{array}$} \\
\hline \multicolumn{9}{|c|}{$100 \mathrm{mg} / \mathrm{dL}$} \\
\hline Well \# & $\begin{array}{c}\Delta \mathrm{mg} / \mathrm{dL} \\
\left(\mathrm{t}_{0}-\mathbf{t}_{72}\right)\end{array}$ & $\begin{array}{l}\operatorname{Avg} \Delta \\
\mathbf{m g} / \mathbf{d L} \\
\left(\mathbf{t}_{0}-\mathbf{t}_{\mathbf{7 2}}\right)\end{array}$ & $\begin{array}{c}\Delta \mathrm{mg} / \mathrm{dL} \\
\left(\mathrm{t}_{0}-\mathbf{t}_{24}\right)\end{array}$ & $\begin{array}{l}\operatorname{Avg} \Delta \\
\mathbf{m g} / \mathbf{d L} \\
\left(\mathbf{t}_{0}-\mathbf{t}_{24}\right)\end{array}$ & $\begin{array}{r}\Delta \mathbf{m g} / \mathbf{d L} \\
\left(\mathbf{t}_{24}-\mathbf{t}_{48}\right)\end{array}$ & $\begin{array}{c}\operatorname{Avg} \Delta \\
\mathbf{m g} / \mathbf{d L} \\
\left(\mathbf{t}_{24}-\mathbf{t}_{48}\right)\end{array}$ & $\begin{array}{c}\Delta \mathrm{mg} / \mathrm{dL} \\
\left(\mathbf{t}_{48}-\mathbf{t}_{72}\right)\end{array}$ & $\begin{array}{c}\operatorname{Avg} \Delta \\
\mathbf{m g} / \mathbf{d L} \\
\left(\mathbf{t}_{48}-\mathbf{t}_{72}\right)\end{array}$ \\
\hline 1 & 15 & \multirow{3}{*}{16.333} & 1 & \multirow{3}{*}{-1.000} & 6 & \multirow{3}{*}{6.667} & 8 & \multirow{3}{*}{10.667} \\
\hline 2 & 15 & & -3 & & 6 & & 12 & \\
\hline 3 & 19 & & -1 & & 8 & & 12 & \\
\hline \multicolumn{9}{|c|}{$400 \mathrm{mg} / \mathrm{dL}$} \\
\hline Well \# & $\begin{array}{c}\Delta \mathrm{mg} / \mathrm{dL} \\
\left(\mathrm{t}_{0}-\mathbf{t}_{72}\right)\end{array}$ & $\begin{array}{c}\operatorname{Avg} \Delta \\
\mathrm{mg} / \mathbf{d L} \\
\left(\mathbf{t}_{0}-\mathbf{t}_{72}\right)\end{array}$ & $\begin{array}{c}\Delta \mathrm{mg} / \mathrm{dL} \\
\left(\mathrm{t}_{0}-\mathbf{t}_{24}\right)\end{array}$ & $\begin{array}{c}\operatorname{Avg} \Delta \\
\mathrm{mg} / \mathrm{dL} \\
\left(\mathbf{t}_{\mathbf{0}}-\mathbf{t}_{24}\right)\end{array}$ & $\begin{array}{l}\Delta \mathrm{mg} / \mathrm{dL} \\
\left(\mathrm{t}_{24}-\mathbf{t}_{48}\right)\end{array}$ & $\begin{array}{c}\operatorname{Avg} \Delta \\
\mathbf{m g} / \mathbf{d L} \\
\left(\mathbf{t}_{24}-\mathbf{t}_{48}\right)\end{array}$ & $\begin{array}{c}\Delta \mathrm{mg} / \mathrm{dL} \\
\left(\mathbf{t}_{48}-\mathbf{t}_{72}\right)\end{array}$ & $\begin{array}{c}\operatorname{Avg} \Delta \\
\mathbf{m g} / \mathbf{d L} \\
\left(\mathbf{t}_{48}-\mathbf{t}_{72}\right)\end{array}$ \\
\hline 1 & 37 & \multirow{3}{*}{39.333} & 1 & \multirow{3}{*}{10.333} & 35 & \multirow{3}{*}{20.000} & 1 & \multirow{3}{*}{9.000} \\
\hline 2 & 36 & & 14 & & 10 & & 12 & \\
\hline 3 & 45 & & 16 & & 15 & & 14 & \\
\hline
\end{tabular}


Table 38 - HUVEC Glucose Consumption Comparative Study Experiment \#2

\begin{tabular}{|c|c|c|c|c|}
\hline \multicolumn{5}{|c|}{ HUVEC Glucose Consumption Comparative Study Experiment \#2 } \\
\hline \multirow{2}{*}{ Time } & $\begin{array}{c}\text { Expected Glucose } \\
\text { Concentration } \\
(\mathbf{m g} / \mathbf{d L})\end{array}$ & Glucose Measurements via Accu-Check Glucose Meter (mg/dL) \\
\cline { 3 - 5 } & 100 & Well \#1 & Well \#2 & Well \#3 \\
\hline \multirow{2}{*}{0} & 400 & 263 & 91 & 90 \\
\cline { 2 - 5 } & 100 & 85 & 288 & 296 \\
\hline \multirow{2}{*}{24} & 400 & 277 & 79 & 81 \\
\cline { 2 - 5 } & 100 & 86 & 79 & 78 \\
\hline \multirow{2}{*}{48} & 400 & 274 & 267 & 266 \\
\hline \multirow{2}{*}{72} & 100 & 79 & 80 & 74 \\
\cline { 2 - 5 } & 400 & 261 & 259 & 257 \\
\hline
\end{tabular}

Table 39 - HUVEC Glucose Consumption Comparative Study Experiment \#2 - Well $\Delta \mathrm{mg} / \mathrm{dL}$ per Time Chart

\begin{tabular}{|c|c|c|c|c|c|c|c|c|}
\hline \multicolumn{9}{|c|}{$\begin{array}{l}\text { HUVEC Glucose Consumption Comparative Study Experiment \#2 - } \\
\text { Well } \Delta \text { mg/dL per Time Chart }\end{array}$} \\
\hline \multicolumn{9}{|c|}{$100 \mathrm{mg} / \mathrm{dL}$} \\
\hline Well \# & $\begin{array}{c}\Delta \mathrm{mg} / \mathrm{dL} \\
\left(\mathrm{t}_{0}-\mathrm{t}_{72}\right)\end{array}$ & $\begin{array}{l}\operatorname{Avg} \Delta \\
\mathbf{m g} / \mathbf{d L} \\
\left(\mathbf{t}_{0}-\mathbf{t}_{72}\right)\end{array}$ & $\begin{array}{c}\Delta \mathrm{mg} / \mathrm{dL} \\
\left(\mathrm{t}_{0}-\mathbf{t}_{24}\right)\end{array}$ & $\begin{array}{c}\operatorname{Avg} \Delta \\
\mathbf{m g} / \mathbf{d L} \\
\left(\mathbf{t}_{0}-\mathbf{t}_{24}\right)\end{array}$ & $\begin{array}{c}\Delta \mathrm{mg} / \mathrm{dL} \\
\left(\mathbf{t}_{24}-\mathbf{t}_{48}\right)\end{array}$ & $\begin{array}{c}\operatorname{Avg} \Delta \\
\mathrm{mg} / \mathrm{dL} \\
\left(\mathbf{t}_{24}-\mathbf{t}_{48}\right)\end{array}$ & $\begin{array}{c}\Delta \mathrm{mg} / \mathrm{dL} \\
\left(\mathbf{t}_{\mathbf{4 8}}-\mathbf{t}_{72}\right)\end{array}$ & $\begin{array}{c}\operatorname{Avg} \Delta \\
\operatorname{mg} / d L \\
\left(\mathbf{t}_{48}-\mathbf{t}_{\mathbf{7 2}}\right)\end{array}$ \\
\hline 1 & 15 & \multirow{3}{*}{14.000} & 9 & \multirow{3}{*}{10.000} & -1 & \multirow{3}{*}{0.667} & 7 & \multirow{3}{*}{3.333} \\
\hline 2 & 11 & & 12 & & 0 & & -1 & \\
\hline 3 & 16 & & 9 & & 3 & & 4 & \\
\hline \multicolumn{9}{|c|}{$400 \mathrm{mg} / \mathrm{dL}$} \\
\hline Well \# & $\begin{array}{c}\Delta \mathrm{mg} / \mathrm{dL} \\
\left(\mathrm{t}_{0}-\mathbf{t}_{72}\right)\end{array}$ & $\begin{array}{l}\operatorname{Avg} \Delta \\
\mathbf{m g} / \mathbf{d L} \\
\left(\mathbf{t}_{0}-\mathbf{t}_{72}\right)\end{array}$ & $\begin{array}{c}\Delta \mathrm{mg} / \mathrm{dL} \\
\left(\mathbf{t}_{0}-\mathbf{t}_{24}\right)\end{array}$ & $\begin{array}{l}\operatorname{Avg} \Delta \\
\mathbf{m g} / \mathbf{d L} \\
\left(\mathbf{t}_{0}-\mathbf{t}_{24}\right)\end{array}$ & $\begin{array}{c}\Delta \mathrm{mg} / \mathrm{dL} \\
\left(\mathbf{t}_{24}-\mathbf{t}_{48}\right)\end{array}$ & $\begin{array}{c}\operatorname{Avg} \Delta \\
\operatorname{mg} / d L \\
\left(\mathbf{t}_{24}-\mathbf{t}_{48}\right)\end{array}$ & $\begin{array}{c}\Delta \mathrm{mg} / \mathrm{dL} \\
\left(\mathbf{t}_{48}-\mathbf{t}_{72}\right)\end{array}$ & $\begin{array}{c}\operatorname{Avg} \Delta \\
\operatorname{mg} / d L \\
\left(t_{48}-t_{72}\right)\end{array}$ \\
\hline 1 & 2 & \multirow{3}{*}{23.333} & -14 & \multirow{3}{*}{12.000} & 3 & \multirow{3}{*}{1.333} & 13 & \multirow{3}{*}{10.000} \\
\hline 2 & 29 & & 42 & & -21 & & 8 & \\
\hline 3 & 39 & & 8 & & 22 & & 9 & \\
\hline
\end{tabular}


Table 40 - BAEC Glucose Consumption Comparative Study Experiment \#3

\begin{tabular}{|c|c|c|c|c|}
\hline \multirow{5}{*}{ Time } & \multirow{5}{*}{$\begin{array}{c}\text { Expected Glucose } \\
\text { Concentration } \\
(\mathbf{m g} / \mathbf{d L})\end{array}$} & Well \#1 & Well \#2 & Well \#3 \\
\cline { 3 - 5 } & 100 & 74 & 82 & 84 \\
\hline \multirow{2}{*}{0} & 400 & 367 & 364 & 357 \\
\cline { 2 - 5 } & 100 & 77 & 79 & 72 \\
\hline \multirow{2}{*}{24} & 400 & 311 & 324 & 276 \\
\cline { 2 - 5 } & 100 & 76 & 70 & 80 \\
\hline \multirow{2}{*}{48} & 400 & 306 & 313 & 269 \\
\cline { 2 - 5 } & 100 & 75 & 71 & 81 \\
\hline \multirow{2}{*}{72} & 400 & 292 & 293 & 266 \\
\hline
\end{tabular}

Table 41 - BAEC Glucose Consumption Comparative Study Experiment \#3 - Well $\Delta \mathrm{mg} / \mathrm{dL}$ per Time Chart

\begin{tabular}{|c|c|c|c|c|c|c|c|c|}
\hline \multicolumn{9}{|c|}{$\begin{array}{l}\text { BAEC Glucose Consumption Comparative Study Experiment \#3 - } \\
\text { Well } \Delta \text { mg/dL per Time Chart }\end{array}$} \\
\hline \multicolumn{9}{|c|}{$100 \mathrm{mg} / \mathrm{dL}$} \\
\hline Well \# & $\begin{array}{c}\Delta \mathbf{m g} / \mathbf{d L} \\
\left(\mathbf{t}_{0}-\mathbf{t}_{72}\right)\end{array}$ & $\begin{array}{l}\operatorname{Avg} \Delta \\
\mathbf{m g} / \mathbf{d L} \\
\left(\mathbf{t}_{0}-\mathbf{t}_{72}\right)\end{array}$ & $\begin{array}{c}\Delta \mathbf{m g} / \mathbf{d L} \\
\left(\mathbf{t}_{\mathbf{0}}-\mathbf{t}_{24}\right)\end{array}$ & $\begin{array}{c}\operatorname{Avg} \Delta \\
\operatorname{mg} / d L \\
\left(t_{0}-t_{24}\right)\end{array}$ & $\begin{array}{l}\Delta \mathrm{mg} / \mathrm{dL} \\
\left(\mathbf{t}_{24}-\mathbf{t}_{48}\right)\end{array}$ & $\begin{array}{c}\operatorname{Avg} \Delta \\
\operatorname{mg} / d L \\
\left(\mathbf{t}_{24}-\mathbf{t}_{48}\right)\end{array}$ & $\begin{array}{l}\Delta \mathbf{m g} / \mathbf{d L} \\
\left(\mathbf{t}_{48}-\mathbf{t}_{72}\right)\end{array}$ & $\begin{array}{c}\operatorname{Avg} \Delta \\
\operatorname{mg} / d L \\
\left(t_{48}-t_{72}\right)\end{array}$ \\
\hline 1 & -1 & \multirow{3}{*}{4.333} & -3 & \multirow{3}{*}{4.000} & 1 & \multirow{3}{*}{0.667} & 1 & \multirow{3}{*}{-0.333} \\
\hline 2 & 11 & & 3 & & 9 & & -1 & \\
\hline 3 & 3 & & 12 & & -8 & & -1 & \\
\hline \multicolumn{9}{|c|}{$400 \mathrm{mg} / \mathrm{dL}$} \\
\hline Well \# & $\begin{array}{c}\Delta \mathbf{m g} / \mathbf{d L} \\
\left(\mathbf{t}_{\mathbf{0}}-\mathbf{t}_{72}\right)\end{array}$ & $\begin{array}{c}\operatorname{Avg} \Delta \\
\mathbf{m g} / \mathbf{d L} \\
\left(\mathbf{t}_{\mathbf{0}}-\mathbf{t}_{\mathbf{7 2}}\right)\end{array}$ & $\begin{array}{c}\Delta \mathbf{m g} / \mathbf{d L} \\
\left(\mathbf{t}_{\mathbf{0}}-\mathbf{t}_{24}\right)\end{array}$ & $\begin{array}{c}\operatorname{Avg} \Delta \\
\mathbf{m g} / \mathbf{d L} \\
\left(\mathbf{t}_{\mathbf{0}}-\mathbf{t}_{24}\right)\end{array}$ & $\begin{array}{l}\Delta \mathbf{m g} / \mathbf{d L} \\
\left(\mathbf{t}_{24}-\mathbf{t}_{48}\right)\end{array}$ & $\begin{array}{c}\operatorname{Avg} \Delta \\
\mathbf{m g} / \mathbf{d L} \\
\left(\mathbf{t}_{24}-\mathbf{t}_{48}\right)\end{array}$ & $\begin{array}{l}\Delta \mathbf{m g} / \mathbf{d L} \\
\left(\mathbf{t}_{48}-\mathbf{t}_{72}\right)\end{array}$ & $\begin{array}{c}\operatorname{Avg} \Delta \\
\mathbf{m g} / \mathbf{d L} \\
\left(\mathbf{t}_{48}-\mathbf{t}_{\mathbf{7 2}}\right)\end{array}$ \\
\hline 1 & 75 & \multirow{3}{*}{79.000} & 56 & \multirow{3}{*}{48.000} & 5 & \multirow{3}{*}{7.667} & 14 & \multirow{3}{*}{12.333} \\
\hline 2 & 71 & & 40 & & 11 & & 20 & \\
\hline 3 & 91 & & 81 & & 7 & & 3 & \\
\hline
\end{tabular}


Table 42 - HUVEC Glucose Consumption Comparative Study Experiment \#3

\begin{tabular}{|c|c|c|c|c|}
\hline \multicolumn{5}{|c|}{ HUVEC Glucose Consumption Comparative Study Experiment \#3 } \\
\hline \multirow{2}{*}{ Time } & $\begin{array}{c}\text { Expected Glucose } \\
\text { Concentration } \\
(\mathbf{m g} / \mathbf{d L})\end{array}$ & Glucose Measurements via Accu-Check Glucose Meter (mg/dL) \\
\cline { 3 - 5 } & 100 & 85 & Well \#2 & Well \#3 \\
\hline \multirow{2}{*}{0} & 400 & 386 & 86 & 82 \\
\cline { 2 - 5 } & 100 & 75 & 353 & 319 \\
\hline \multirow{2}{*}{24} & 400 & 316 & 318 & 75 \\
\cline { 2 - 5 } & 100 & 68 & 72 & 286 \\
\hline \multirow{2}{*}{48} & 400 & 313 & 306 & 269 \\
\cline { 2 - 5 } & 100 & 69 & 68 & 75 \\
\hline \multirow{2}{*}{72} & 400 & 288 & 298 & 265 \\
\hline
\end{tabular}

Table 43 - HUVEC Glucose Consumption Comparative Study Experiment \#3 - Well $\Delta \mathrm{mg} / \mathrm{dL}$ per Time Chart

\begin{tabular}{|c|c|c|c|c|c|c|c|c|}
\hline \multicolumn{9}{|c|}{$\begin{array}{l}\text { HUVEC Glucose Consumption Comparative Study Experiment \#3 - } \\
\text { Well } \Delta \text { mg/dL per Time Chart }\end{array}$} \\
\hline \multicolumn{9}{|c|}{$100 \mathrm{mg} / \mathrm{dL}$} \\
\hline Well \# & $\begin{array}{c}\Delta \mathrm{mg} / \mathrm{dL} \\
\left(\mathbf{t}_{0}-\mathbf{t}_{72}\right)\end{array}$ & $\begin{array}{l}\operatorname{Avg} \Delta \\
\mathbf{m g} / d L \\
\left(t_{0}-t_{72}\right)\end{array}$ & $\begin{array}{c}\Delta \mathrm{mg} / \mathrm{dL} \\
\left(\mathbf{t}_{0}-\mathbf{t}_{24}\right)\end{array}$ & $\begin{array}{l}\operatorname{Avg} \Delta \\
\mathbf{m g} / d L \\
\left(t_{0}-t_{24}\right)\end{array}$ & $\begin{array}{l}\Delta \mathrm{mg} / \mathrm{dL} \\
\left(\mathrm{t}_{24}-\mathbf{t}_{48}\right)\end{array}$ & $\begin{array}{c}\operatorname{Avg} \Delta \\
\mathbf{m g} / \mathbf{d L} \\
\left(\mathbf{t}_{24}-\mathbf{t}_{48}\right)\end{array}$ & $\begin{array}{l}\Delta \mathrm{mg} / \mathrm{dL} \\
\left(\mathrm{t}_{\mathbf{4 8}}-\mathrm{t}_{\mathbf{7 2}}\right)\end{array}$ & $\begin{array}{c}\operatorname{Avg} \Delta \mathbf{m g} / \mathbf{d L} \\
\quad\left(\mathbf{t}_{48}-\mathbf{t}_{72}\right)\end{array}$ \\
\hline 1 & 16 & \multirow{3}{*}{13.667} & 10 & \multirow{3}{*}{5.667} & 7 & \multirow{3}{*}{6.000} & -1 & \multirow{3}{*}{2.000} \\
\hline 2 & 18 & & 0 & & 14 & & 4 & \\
\hline 3 & 7 & & 7 & & -3 & & 3 & \\
\hline \multicolumn{9}{|c|}{$400 \mathrm{mg} / \mathrm{dL}$} \\
\hline Well \# & $\begin{array}{c}\Delta \mathrm{mg} / \mathrm{dL} \\
\left(\mathbf{t}_{0}-\mathbf{t}_{72}\right)\end{array}$ & $\begin{array}{l}\operatorname{Avg} \Delta \\
\mathbf{m g} / \mathbf{d L} \\
\left(\mathbf{t}_{\mathbf{0}}-\mathbf{t}_{72}\right)\end{array}$ & $\begin{array}{c}\Delta \mathrm{mg} / \mathrm{dL} \\
\left(\mathrm{t}_{0}-\mathbf{t}_{24}\right)\end{array}$ & $\begin{array}{l}\operatorname{Avg} \Delta \\
\mathbf{m g} / \mathbf{d L} \\
\left(\mathbf{t}_{\mathbf{0}}-\mathbf{t}_{24}\right)\end{array}$ & $\begin{array}{l}\Delta \mathrm{mg} / \mathrm{dL} \\
\left(\mathrm{t}_{24}-\mathbf{t}_{48}\right)\end{array}$ & $\begin{array}{c}\operatorname{Avg} \Delta \\
\mathbf{m g} / \mathbf{d L} \\
\left(\mathbf{t}_{24}-\mathbf{t}_{48}\right)\end{array}$ & $\begin{array}{l}\Delta \mathrm{mg} / \mathrm{dL} \\
\left(\mathrm{t}_{48}-\mathrm{t}_{72}\right)\end{array}$ & $\begin{array}{c}\operatorname{Avg} \Delta \mathbf{m g} / \mathbf{d L} \\
\left(\mathbf{t}_{48}-\mathbf{t}_{72}\right)\end{array}$ \\
\hline 1 & 98 & \multirow{3}{*}{69.000} & 70 & \multirow{3}{*}{46.000} & 3 & \multirow{3}{*}{10.667} & 25 & \multirow{3}{*}{12.333} \\
\hline 2 & 55 & & 35 & & 12 & & 8 & \\
\hline 3 & 54 & & 33 & & 17 & & 4 & \\
\hline
\end{tabular}




\section{Appendix D: Protocol for the Development of Sterile 400 mg/dL Hyperglycemic Cell Media Solution}

- Materials

○ $100 \mathrm{~mL}$ complete cell media

○ 0.30 g Sigma G6152 D-(+)-Glucose

- Aviva Accu-Chek Blood Glucose Meter \& Test Strips

Note: Use two different sets of blood glucose test strips - one for use in a nonsterile environment and one for use within a sterile environment.

○ $50 \mathrm{~mL}$ graduate cylinder

○ $250 \mathrm{~mL}$ beaker

○ Magnetic stir bar \& stir plate

○ Balance

○ Four $50 \mathrm{~mL}$ conicals

○ Two $10 \mathrm{~mL}$ syringes

○ Four $0.22 \mu \mathrm{m}$ syringe filters

- Procedure

○ Development of $400 \mathrm{mg} / \mathrm{dL}$ Hyperglycemic Cell Media Solution

1. Weigh $0.3 \mathrm{~g}$ of Sigma G6152 D-(+)-Glucose

2. Using a $50 \mathrm{~mL}$ graduated cylinder, measure $100 \mathrm{~mL}$ of cell media into a $250 \mathrm{~mL}$ beaker.

3. Add all 0.3g of Sigma G6152 D-(+)-Glucose to the $100 \mathrm{~mL}$ of cell media.

4. Place magnetic stir bar into beaker and stir solution on stir plate for at least six hours at $25^{\circ} \mathrm{C}$ to allow uniform mixing.

5. Using Aviva Accu-Chek blood glucose meter, record the glucose concentration of the glycemic cell media solution.

6. Transfer solution from $250 \mathrm{~mL}$ beaker to two $50 \mathrm{~mL}$ conicals.

○ Sterilization of $400 \mathrm{mg} / \mathrm{dL}$ Hyperglycemic Cell Media Solution

1. Ensure hands are washed with gloves on. Spray gloves with $70 \%$ IPA. 
2. Spray inside of laminar flow hood with $70 \%$ IPA.

3. Keep sterile packaged syringes and syringe filters inside packaging and place inside hood.

4. Sterilize two brand new $50 \mathrm{~mL}$ conicals and place inside hood.

5. Sterilize $50 \mathrm{~mL}$ conical with $400 \mathrm{mg} / \mathrm{dL}$ hyperglycemic cell media solution (cap closed) with $70 \%$ IPA.

6. Open syringes and syringe filters inside hood. Do not allow the tips to touch the outside of the packing. Discard of packaging.

7. Uptake $10 \mathrm{~mL}$ of $400 \mathrm{mg} / \mathrm{dL}$ hyperglycemic cell media solution into $10 \mathrm{~mL}$ syringe.

8. Place $22 \mu \mathrm{m}$ syringe filter on end of syringe.

9. Slowly transfer $10 \mathrm{~mL}$ of solution through filter into a brand new conical. This solution is now sterile.

10. Repeat steps 8-10 for all $100 \mathrm{~mL}$ of unsterile $400 \mathrm{mg} / \mathrm{dL}$ hyperglycemic solution. Use second conical when necessary.

11. Once all media has been sterilized into sterile conicals, tightly cap the conicals inside laminar flow hood and store sterilized $400 \mathrm{mg} / \mathrm{dL}$ solution at $5-10^{\circ} \mathrm{C}$. 


\section{Appendix E: Protocol Using Aviva Accu-Chek Blood}

\section{Glucose Meter}

*All images provided from Aviva Accu-Chek Owner's Booklet

1. Overview of the blood glucose meter

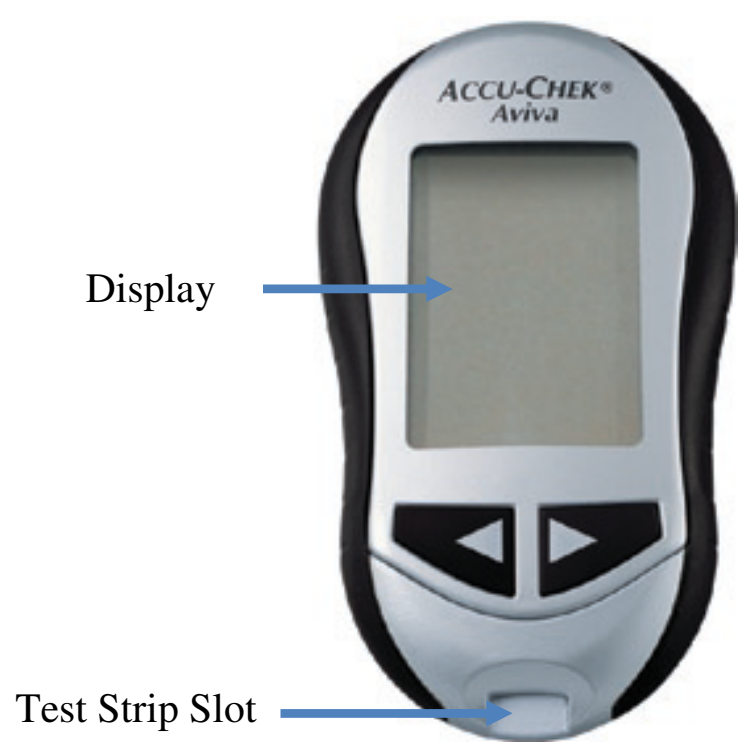

Power Button 


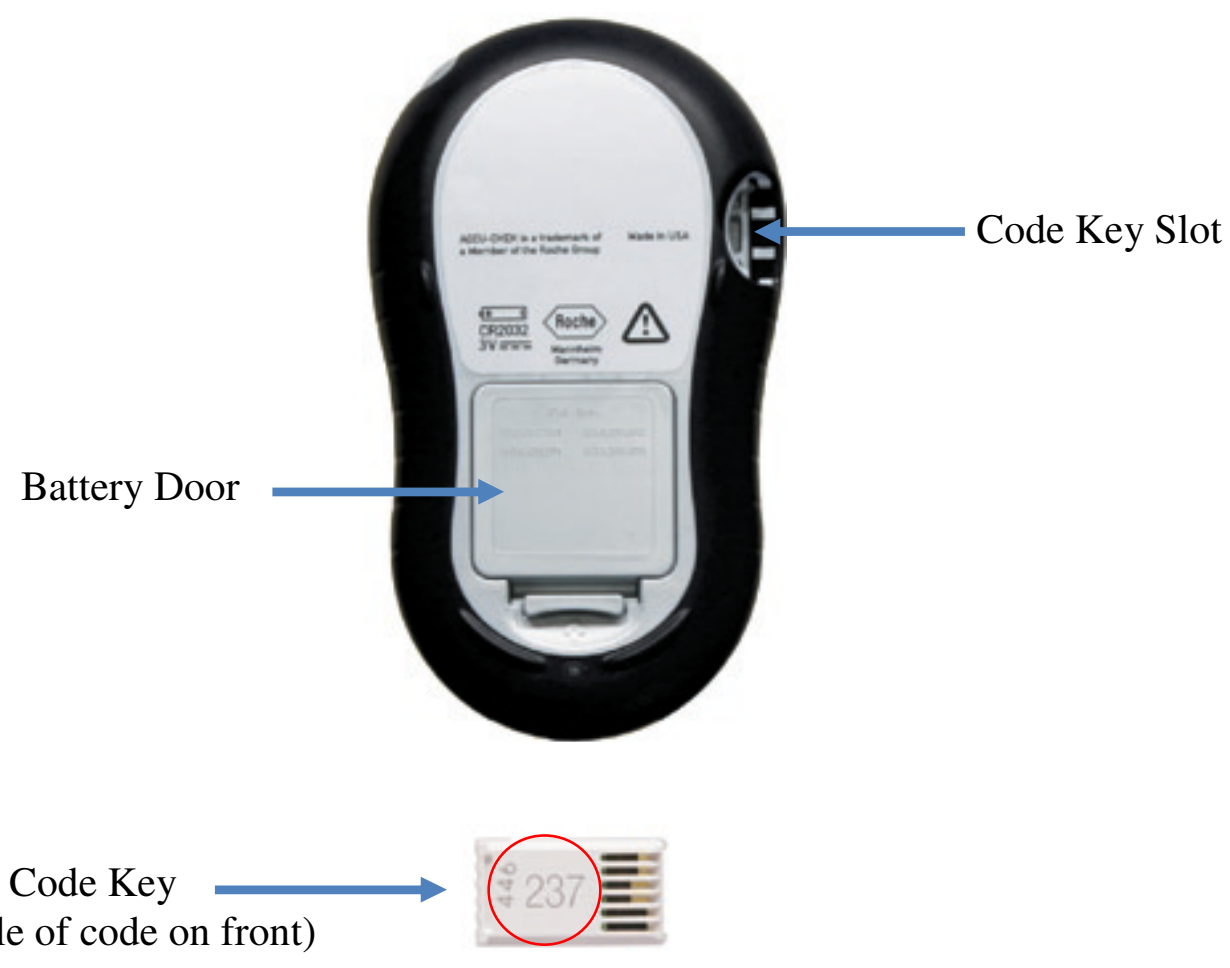

(example of code on front)
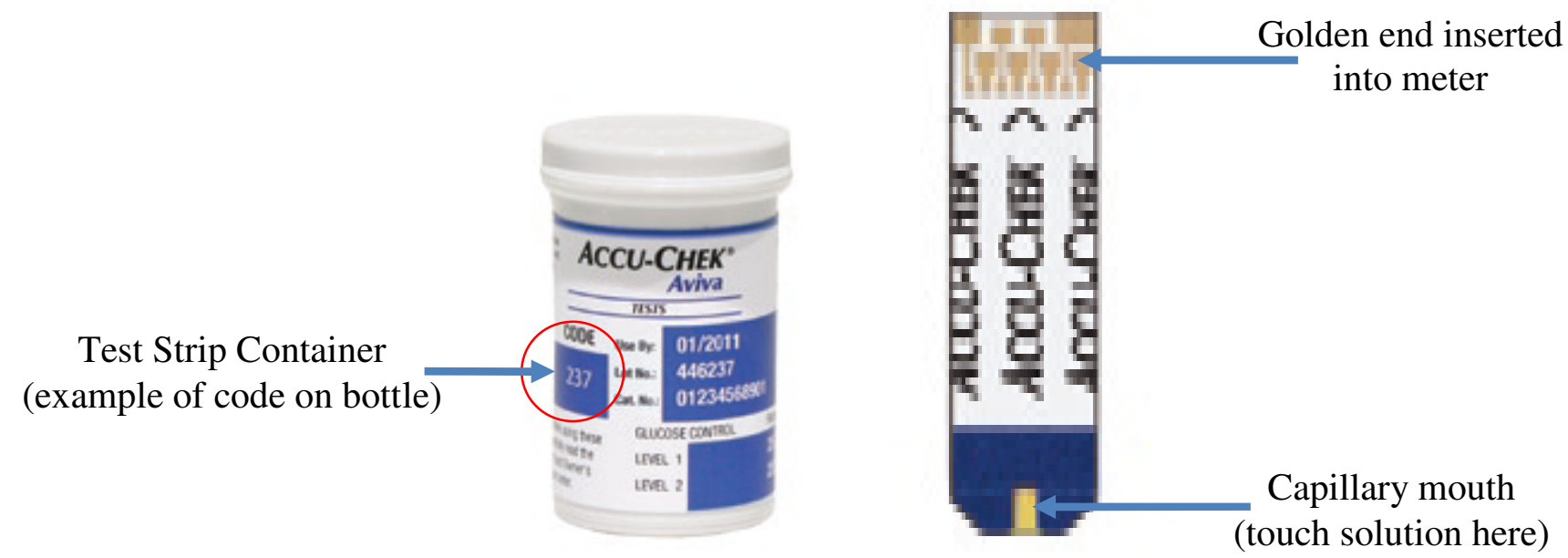
2. Coding the Meter

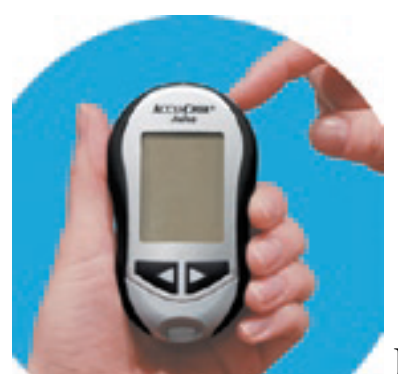

Ensure that the meter is off
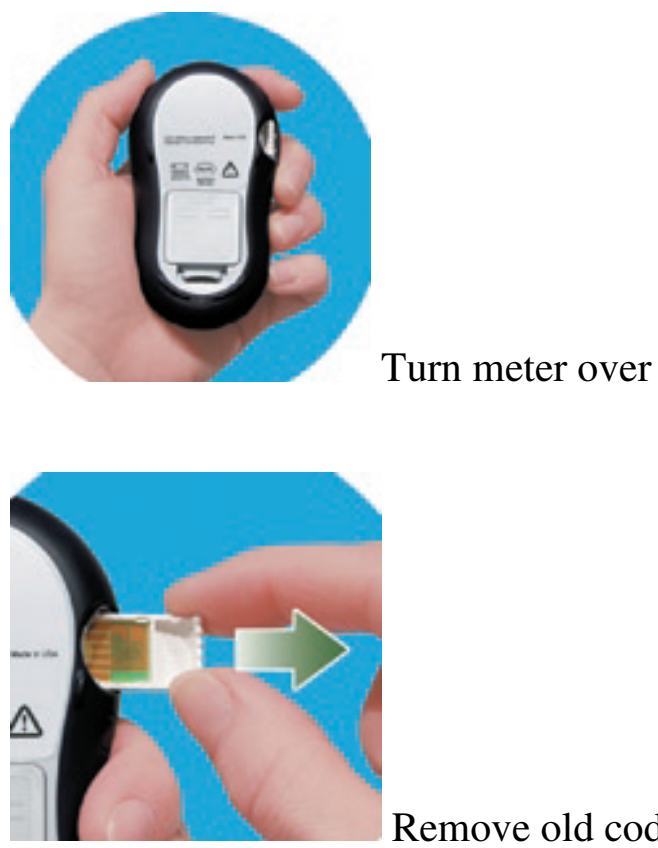

Remove old code key (if there is one in the meter) and discard it

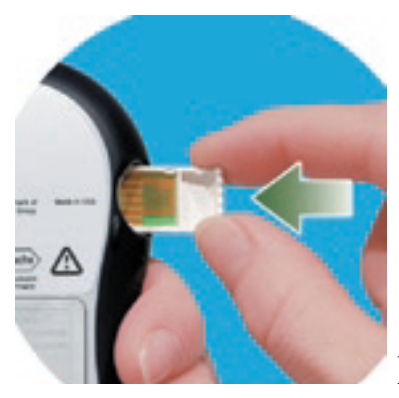

Insert new code key for test strip container (ensure that the code is facing away from you). Push in the code key until it stops. Leave code key in meter until a new container of test strips (with a different code key) is being used. 
3. Running a Glucose Concentration Test

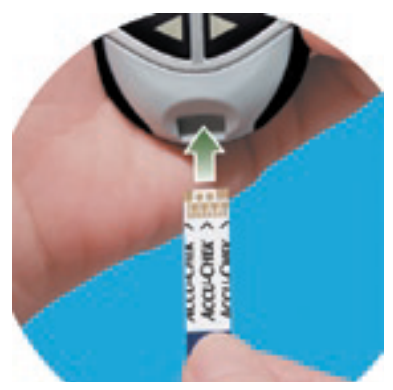

Insert a test strip into the test strip slot of the blood glucose meter.

The meter will automatically turn on.

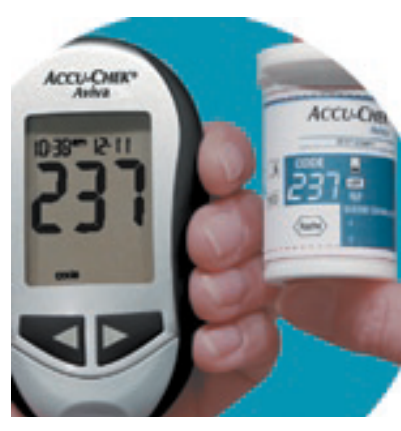

Ensure that the code on the display shows the same code that is on the container. If missed, remove the test strip and reinsert it to observe the code on the display screen.

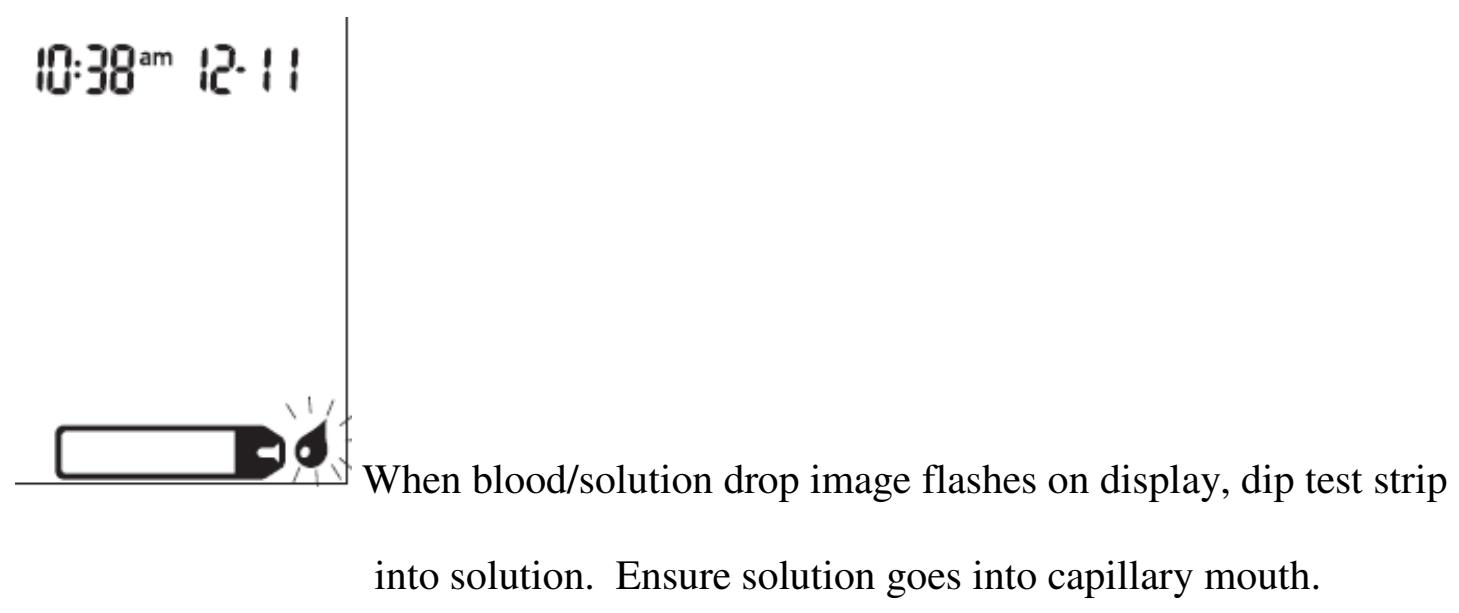



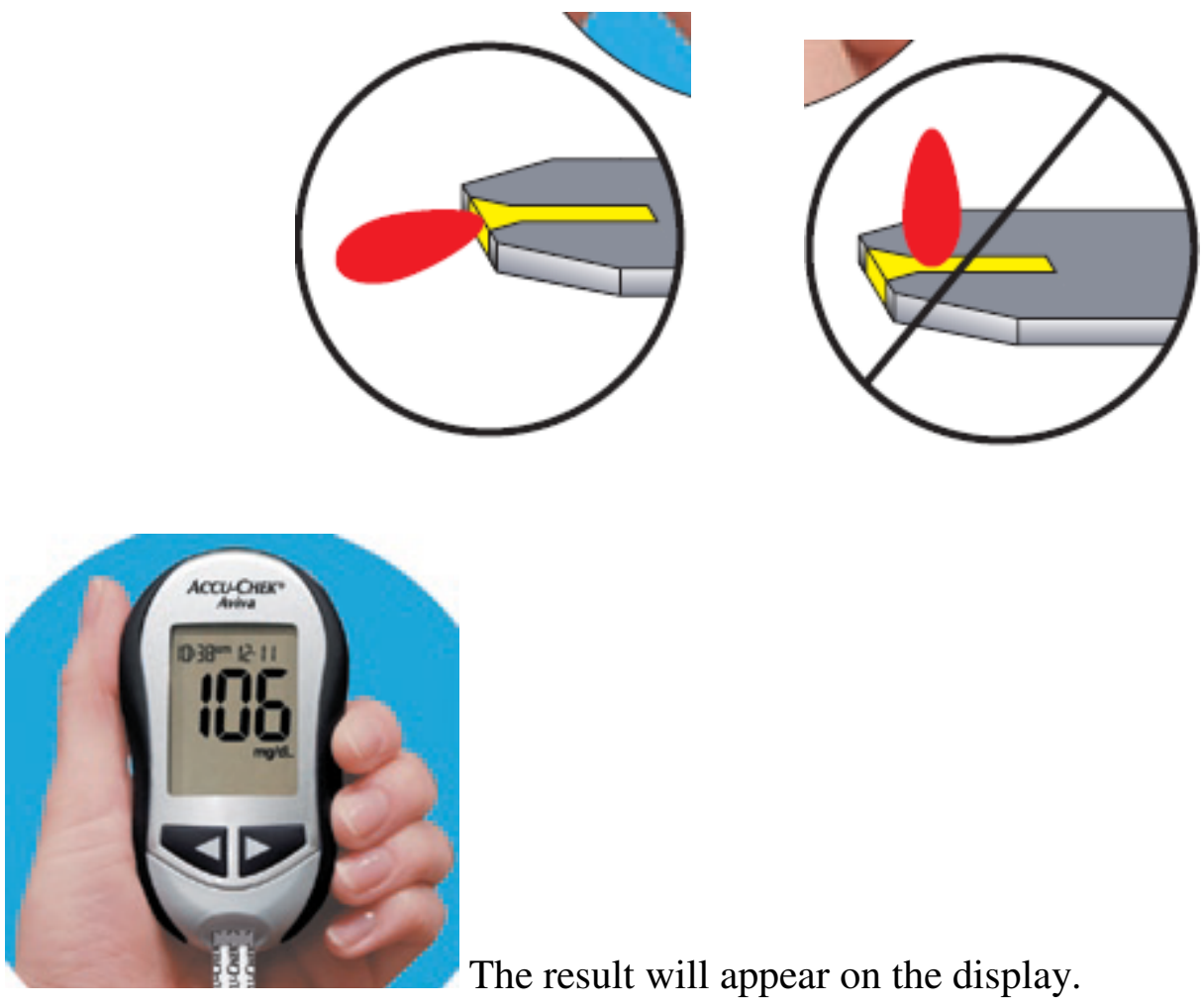